\title{
RADIO AND X-RAY DIAGNOSTICS OF ENERGY RELEASE IN SOLAR FLARES
}

\author{
Bin Chen \\ Jiangxi, China
}

\begin{abstract}
B.S., Peking University, 2005
M.S., University of Chinese Academy of Sciences, 2008

M.S., University of Virginia, 2010
A Dissertation Presented to the Graduate
Faculty of the University of Virginia
in Candidacy for the Degree of
Doctor of Philosophy
Department of Astronomy
University of Virginia
August, 2013

Timothy S. Bastian

Zhi-Yun Li

Philip L. Arras

Robert E. Johnson

Dale E. Gary 
To Yufei 


\begin{abstract}
Solar flares involve catastrophic release of magnetic energy previously stored in the Sun's corona. This dissertation focuses on studies of radio and X-ray emissions as diagnostics of energy release processes in flares and the environment in which they occur.

A major part of the dissertation is exploiting spatially resolved dynamic spectroscopy to study coherent radio bursts. The Frequency-Agile Solar Radiotelescope Subsystem Testbed, a three-element radio interferometer, provides the first opportunity of doing such studies on zebra-pattern bursts. The observations allow us to identify the relevant emission mechanism, enabling diagnostics of the plasma parameters in the source. With the help of coronal magnetic field extrapolations, the source is placed into a three-dimensional magnetic field configuration and its relation to the energy release is clarified. The next part of the dissertation discusses the "solar mode" commissioning of the recently upgraded Karl G. Jansky Very Large Array (VLA). As a general purpose telescope, special provisions should be made for the $V L A$ to enable solar observations. Based on the test results on the $V L A$ 's hardware, solar observing and calibration strategies are developed. Now the $V L A$ serves as the most powerful radio telescope currently available for solar observing, capable of performing simultaneous imaging and dynamic spectroscopy over a large bandwidth at high spatial, spectral, and temporal resolution. The upgraded $V L A$ is used to observe decimetric type III radio bursts, which are the radio signature of propagating fast electron beams produced in flares. The new observing technique allows detailed trajectories of these electron beams to be derived. Combined with multi-wavelength observations, the properties of the energy release site, electron beams, and the surrounding coronal medium are deduced.
\end{abstract}


The dissertation also presents a study on coronal hard X-ray $/ \gamma$-ray sources. Rather extreme conditions are needed to account for some observed coronal hard X-ray $/ \gamma$ ray sources using the usually-assumed non-thermal bremsstrahlung emission. This study investigates whether inverse Compton scattering could be an alternative emission mechanism for these sources, which would open a new window in diagnosing the flare energy release. 


\section{Acknowledgements}

First of all, I would like to sincerely thank my advisor, Tim Bastian, an admirable scientist and a wonderful mentor, for his advice and guidance throughout the five years of my graduate studies at the University of Virginia. I have learnt a great deal from Tim, not only in solar physics, but also in how to become a better scientist. I hope that I could be as enthusiastic, energetic, and knowledgeable as Tim someday in the future. In addition to a good advisor, he is also a good friend who helped me through these years in many aspects of my life. He and his wife, Ruta, have always treated us like a family. I will never forget their warmness and kindness.

Many thanks to my committee members - Zhi-Yun Li, Phil Arras, Bob Johnson and Dale Gary - for their advice and support from the thesis proposal to the defense. My special gratitude goes to Dale Gary, who was also one of my closest colleagues. I was fortunate enough to get to know him when I was applying for graduate schools, and received great help from him on instrumentation and data analysis of the OVSA and FST which led to Chapter 2 of this thesis.

I will forever be thankful to my former research advisor, Yihua Yan. Yihua was the reason why I decided to pursue a career in solar physics. He was and remains very helpful in providing advices during my graduate school career. I also thank all the members of the Solar Radio Research Group at NAOC. We have spent a great time together in Beijing, and I will always cherish our friendship.

I feel deeply grateful to the UVa Astronomy Department and NRAO for providing the abundant resources and wonderful academic environment. I owe my thanks to all the UVa Astro faculty and my fellow graduate students. They made my years in Charlottesville to be one of my fondest memories. Special thanks to my dear classmates. We have had a wonderful time on late-night homework parties, giving 
each other nick names, and learning good/bad words in different languages.

I thank the many great people I have met at NRAO/DSOC during my VLA Resident Shared Risk Observing program in Socorro, NM, without whom the success of the RSRO program would be impossible: Rick Perley, Michael Rupen, Ken Sowinski, Joseph McMullin, Joan Wrobel, Bryan Butler, Keith Morris, Claire Chandler, George Moellenbrock, Urvashi Rau, and many others.

My sincere thanks also goes to the multitude of scientists who have helped me and/or shared their experience with me during these years: Stephen White, Ju Jing, Säm Krucker, Arnold Benz, Hugh Hudson, Henry Aurass, Gregory Fleishman, Gelu Nita, Thomas Wiegelmann, Bob Lin, Haimin Wang, Vahé Petrosian, Iver Cairns.

I am especially grateful to Yufei He, my beloved wife, for her continual love and encouragement. Without her support, I would have been lost in direction. I am constantly amazed by her ability of remembering literally everything that are important and reminding me in time, by her extraordinary sense of direction (she is a human navigator!), by her unbeatable logical thinking skills, and by the mouthwatering dishes she cooks. Special thanks to my lovely baby girl, Yulan, without her ultimate cuteness I may have finished the thesis earlier. Yufei and Yulan are the most important pieces in my life, who truly give me the greatest joy I could ever imagine. Thanks to my parents for all of their love and support throughout my life. I always know that no matter what happened, they would be there for me.

Finally, I would like to acknowledge the support by NSF grant AGS-1010652 to the University of Virginia, the support from the NRAO through the Summer Student Research Assistantship and the Resident Shared Risk Observing program, and the International Space Science Institute for their support of the international research team "X-ray and radio diagnostics of energetic electrons in solar flares". 


\section{Table of contents}

List of Figures $\quad$ xi

List of Tables xii

1 General Introduction 1

1.1 Overview of the Sun . . . . . . . . . . . . . . . . . . . 2

1.2 Solar Flares . . . . . . . . . . . . . . . . . . . . . . 7

1.2.1 Overview ......................... 7

1.2.2 The Standard Flare Model . . . . . . . . . . . . . . . . 9

1.2 .3 Radio Emission . . . . . . . . . . . . . . . . . . . . 15

1.2.4 X-ray Emission . . . . . . . . . . . . . . . . . 29

1.3 Instrumentation . . . . . . . . . . . . . . . . . . . . . . . . . . . . . . . . . . . . . .

1.4 Scientific Goal and Dissertation Outline . . . . . . . . . . . . 37

2 Zebra-Pattern Radio Bursts: Coherent Emission From Trapped Electrons $\quad 40$

2.1 Introduction . . . . . . . . . . . . . . . . . 41

2.2 Instrumentation . . . . . . . . . . . . . . . . . . . . . 46

2.3 Observations . . . . . . . . . . . . . . . . . . . . 49

2.3.1 FST ZP Observations ...................... 52

2.3.2 Magnetic Field Configuration . . . . . . . . . . . . . 69

2.4 Discussion . . . . . . . . . . . . . . . . . . . . . . 71

2.5 Conclusion . . . . . . . . . . . . . . . . . 83

3 Karl G. Jansky Very Large Array "Solar Mode" Commissioning 86

3.1 Introduction . . . . . . . . . . . . . . . . . 87

3.2 Hardware Implementation and Tests . . . . . . . . . . . . . . . . . 91

3.2.1 System Linearity . . . . . . . . . . . . . . . . . . . . . . . 91

3.2.2 Gain Reduction . . . . . . . . . . . . . . . . . . 96

3.3 Solar Observing and Calibration Strategies . . . . . . . . . . . . . 100

3.3.1 Solar Observing Strategies . . . . . . . . . . . . . . . . . 102

3.3.2 Solar Data Calibration . . . . . . . . . . . . . . . . . 104 
4 Type III Radio Bursts: Traces of Propagating Electron Beams 111

4.1 Introduction . . . . . . . . . . . . . . . . . . . . . 112

4.2 From Dynamic Spectroscopy and Imaging to Dynamic Imaging Spectroscopy . . . . . . . . . . . . . . . . . . 114

4.3 Observations . . . . . . . . . . . . . . . . . . . . . . . . . . . . . . . . . . . . . . . . . .

4.4 Results and Discussion . . . . . . . . . . . . . . . . 120

4.5 Conclusion . . . . . . . . . . . . . . . . . . . . . 127

5 Hard X-Ray Sources in the Corona: a Study on the Emission Mechanisms

5.1 Introduction . . . . . . . . . . . . . . . . . . 130

5.2 Inverse Compton Scattering Emission . . . . . . . . . . . . . . . . 134

5.2.1 ICS of Photons on an Isotropic Electron Distribution . . . . . 134

5.2.2 ICS of Isotropic Photons on Anisotropic Electron Distributions 146

5.3 Thin-target Non-thermal Bremsstrahlung Emission . . . . . . . . . . 155

5.4 Application to Coronal HXR Observations . . . . . . . . . . . . . 166

5.4.1 Coronal $\gamma$-Ray Sources from Three Powerful Flares . . . . . . 169

5.4.2 HXR Emission from High in the Corona . . . . . . . . . . . . 171

5.4.3 Electron Acceleration in a "Masuda"-like Event . . . . . . . . 174

5.5 Concluding Remarks . . . . . . . . . . . . . . . . . . 176

6 Summary and Future Work 179

6.1 Summary . . . . . . . . . . . . . . . . . . 180

6.2 Future Work . . . . . . . . . . . . . . . . . . . . 183 


\section{List of Figures}

1.1 Sketch of sunspots by Gao Pingzi in 1925. . . . . . . . . . . . . . . . 3

1.2 Temperature and density in the solar atmosphere. . . . . . . . . . . 4

1.3 Structures of the solar corona. . . . . . . . . . . . . . . 6

1.4 Schematic representation of different phases of a solar flare. . . . . . . 10

1.5 Flare observations in X-ray. . . . . . . . . . . . . . . . . . . . . . . . . . . . 12

1.6 The standard flare model. . . . . . . . . . . . . . . . . . . . . . 14

1.7 Typical brightness temperature spectra from thermal bremsstrahlung and non-thermal gyrosynchrotron radiation. . . . . . . . . . . . . . . 21

1.8 Examples of solar coherent radio bursts . . . . . . . . . . . . 25

2.1 Antenna configuration of the FST. . . . . . . . . . . . . . . . 46

2.2 Comparison of OVSA and FST amplitude and phase for cross-calibration. 48

2.3 SXR and radio time profiles of the 2006 December 14 flare. . . . . . . 50

2.4 Incoherent radio source from the OVSA and the (coherent) zebrapattern burst centroid location obtained by the FST superposed on the photospheric magnetogram and the $\mathrm{Ca}$ II $\mathrm{H}$ band image from the SOT aboard Hinode. . . . . . . . . . . . . . . . . . . . . . . 51

2.5 Hinode SOT Ca II H and XRT observations of the 2004 December 14 flare showing the chromospheric and coronal evolution. . . . . . . . 53

2.6 Dynamic spectral properties of the 2006 December 14 zebra-pattern burst observed by the FST. . . . . . . . . . . . . . . . . . . . . 55

2.7 Estimating the source size of the zebra-pattern burst from the FST interferometric observations. . . . . . . . . . . . . . . 57

2.8 The total power dynamic spectrum as well as the "dynamic phase spectrum" of the zebra-pattern burst for the three FST baselines. . . 60

2.9 Amplitude and phase variations of the zebra-pattern burst along the zebra stripes (as a function of time) for the three FST baselines. . . .

2.10 Amplitude and phase variations of the zebra-pattern burst across different zebra stripes (as a function of frequency) for the three FST baselines. . . . . . . . . . . . . . . .

2.11 Absolute source centroid location of the zebra-pattern burst and its variation along and across the zebra stripes. . . . . . . . . . . . 68 
2.12 Deprojected zebra and continuum source locations in the coronal magnetic field derived from a nonlinear force-free field extrapolation method based on the observed photospheric vector magnetic field. . . . . . . .

2.13 Fitting the frequency spacing of the zebra stripes with source plasma parameters based on the DPR model. . . . . . . . . . . . . . 80

2.14 Schematic source model for the zebra-pattern burst observed in the 2006 December 14 flare event. . . . . . . . . . . . . . . . . . . 84

3.1 Overview of $V L A$ 's electronics system . . . . . . . . . . . . . 87

3.2 Compression from VLA's amplifiers . . . . . . . . . . . . . . . . 93

3.3 Compression from $V L A$ 's IF system . . . . . . . . . . . . . . . . . . . 95

3.4 Net phase introduced by the $20 \mathrm{~dB}$ solar attenuators as a function of frequency. . . . . . . . . . . . . . . . .

3.5 Residual phase vs frequency of the $20 \mathrm{~dB}$ solar attenuators after delay corrections. . . . . . . . . . . . . . . . 100

3.6 Phase versus frequency of the T304 downconverter attenuators. . . 101

3.7 Work-flow diagram of $V L A$ data calibration with CASA. . . . . . . . 108

3.8 Bandpass solutions of amplitude using polynomial fits. . . . . . . . . 109

3.9 Bandpass solutions of phase using polynomial fits. . . . . . . . . . . . 110

4.1 Examples of various types of solar coherent radio bursts observed by the VLA on 2012 March 3. . . . . . . . . . . . . . . . . . . . . . 116

4.2 Type IIIdm bursts observed on 2011 November 5 by the $V L A$ during a coronal jet event. . . . . . . . . . . . . . . . . . . . . 118

4.3 STEREO/EUVI B and SDO/AIA images of the type-IIIdm-burstassociated coronal jet. . . . . . . . . . . . . . . . . 119

4.4 Emission centroid locations as a function of frequency of the type IIIdm bursts. . . . . . . . . . . . . . . . . .

4.5 Evolution of the HXR footpoint emission and coronal EUV jet associated with the type IIIdm bursts. . . . . . . . . . . . . . . . . . . . . . 123

4.6 Schematic illustration of the magnetic field evolution, reconnection, coronal EUV jet, and generation of the electron beams and HXR footpoint sources for the 2011 November 5 event. . . . . . . . . . . . . . . 124

5.1 Geometry used to calculate the ICS emission from an anisotropic photon field. . . . . . . . . . . . . . . . . . .

5.2 Illustration of the source geometry for evaluating the ICS emission from an incident optical photon field which fills the half-space above the photosphere.

5.3 ICS photon spectra from an optical photon field upscattered by ultrarelativistic electrons. Effects of assuming different viewing angles and different spectral indices of the electron energy distribution are shown. 140 
5.4 Upscattered photon spectra from incident photons scattered by monoenergetic isotropic electron distributions with different electron energies at different scattering angles using the exact ICS calculation.

5.5 Upscattered photon spectra due to ICS emission in the mildly relativistic regime from isotropic electron distributions with different spectral indices. . . . . . . . . . . . . . . . . . . . 146

5.6 Geometry and angles used to calculate the ICS emission between a unidirectional photon beam and a unidirectional electron beam. . . . 147

5.7 Normalized ICS emission rate at different angles of the source electron relative to the line of sight. . . . . . . . . . . . . . . . . . . . . . . 149

5.8 Upscattered photon spectra due to ICS emission in the ultrarelativistic regime from electron distributions with different types of anisotropies. 150

5.9 Upscattered photon spectra due to ICS emission in the mildly relativistic regime from electron distributions with different types of anisotropies. 151

5.10 Geometry and angles used to calculate the ICS emission between a disk-like electron angular distribution and an isotropic photon field. . 154

5.11 Polar diagram of the normalized emissivity of both bremsstrahlung and ICS radiation from a monodirectional electron as a function of the logarithm of the photon energy $\epsilon_{\gamma}$ and the angle of the LOS relative to the beam direction. . . . . . . . . . . . . . . . . . . . . 158

5.12 Bremsstrahlung photon spectra from electron distributions with different types of anisotropies. . . . . . . . . . . . . . . . . . . . 159

5.13 Comparison of ICS and bremsstrahlung emission from the same double power-law spectrum that breaks down (steepens) at higher energies. . 164

5.14 Calculated HXR photon spectra due to ICS emission from simulated flare EUV/SXR photon spectra to account for two flare events. Spectra due to bremsstrahlung emission from the same electron distribution are also shown for comparison. . . . . . . . . . . . . . . . . . 175 


\section{List of Tables}

1.1 Solar flare classifications . . . . . . . . . . . . . . . . . 8

1.2 Waves in a magnetized plasma . . . . . . . . . . 18

3.1 The effects of solar observing with the $V L A \ldots \ldots \ldots$

3.2 Net delays introduced by the $20 \mathrm{~dB}$ solar attenuators. . . . . . . . 98

5.1 Comparison of fitted HXR $/ \gamma$-ray photon spectral indices . . . . . . 161 
Chapter 1

General Introduction 


\subsection{Overview of the Sun}

Our nearest star, the Sun, is an ordinary star in the Milky Way Galaxy. It has a well-measured mass $M_{\odot} \approx 1.99 \times 10^{33} \mathrm{~g}$, radius $R_{\odot} \approx 6.96 \times 10^{10} \mathrm{~cm}$, and luminosity $L_{\odot} \approx 3.84 \times 10^{33} \mathrm{erg} \mathrm{s}^{-1}$. The chemical composition is $74.9 \%$ hydrogen, $23.8 \%$ helium, and less than $2 \%$ other elements of the mass of the Sun.

From the interior, the Sun can be divided into the core, radiative zone, convective zone, and then the solar atmospheric layers including the photosphere, chromosphere, and corona. The Sun is powered by nuclear processes in its hot core. The photons produced in the core are transported outward diffusively and take about $3 \times 10^{4}$ years to pass through the radiative zone. The radiative zone extends to about $0.7 R_{\odot}$ from the center. Outside that distance lies the convective zone, where the plasma is unstable to convection. As a result, instead of radiation, thermal convection becomes the major means of transferring the energy to the surface of the Sun. Between the radiative zone and the convective zone lies a transition region called the "tachocline". It is the region in which the rotation rate changes from being almost uniform in the radiative zone to being latitude dependent in the convective zone, which results in a strong shear - a condition where successive layers show very different tangential velocities (Spiegel \& Zahn, 1992; Charbonneau et al., 1999). This region is currently believed to be the site of driving the solar magnetic dynamo that produces the Sun's magnetic field (see Fan 2009 for a review).

The photosphere is a thin layer at which the solar atmosphere becomes opaque in optical wavelengths. It has a characteristic temperature of $5800 \mathrm{~K}$. Convective flows can be seen at a variety of sizes known as granules and supergranules. Dark sunspots are often observed in white-light images of the Sun, especially during solar maximum, which are strong magnetic field concentrations emerging from the underlying convec- 


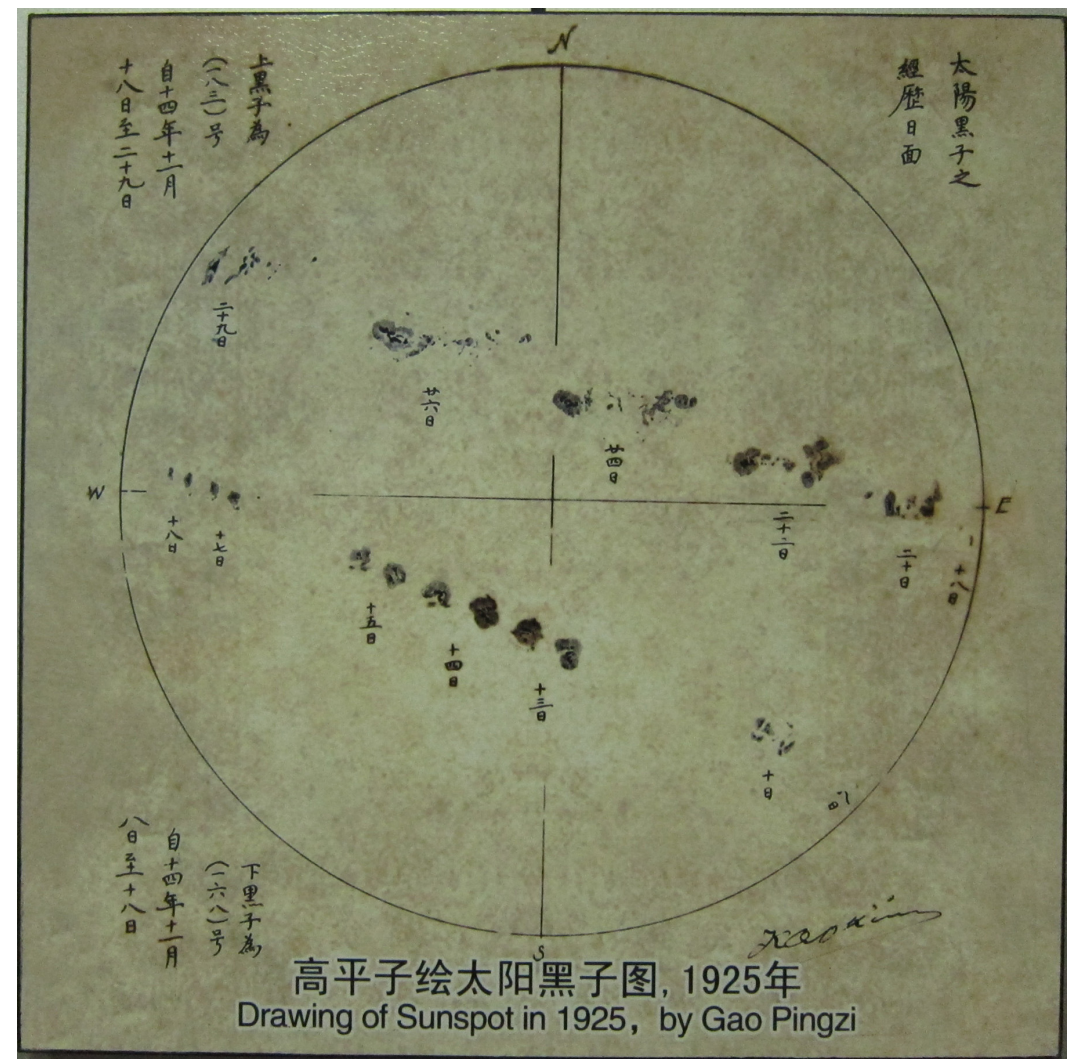

Fig. 1.1. - Sketch of two groups of sunspots by a Chinese Astronomer, Gao Pingzi, from November 8 to 29, 1925. Original drawing is located at the Qingdao Observation Station of the Purple Mountain Observatory. Digital image kindly provided by $\mathrm{Mr}$. Yang Zhang of the Purple Mountain Observatory.

tive zone. The earliest surviving record of sunspot observation was made in $364 \mathrm{BC}$ by Gan De, a Chinese astronomer. After the invention of telescope, sunspots have been frequently observed for more than 400 years since the 17th century. Figure 1.1 shows a sketch of two sunspot groups and their evolution (by Gao Pingzi in November 1925) as an example. Observations of sunspots have been used as an important indication for solar activity variation - the 11-year solar cycle. The layer above the photosphere is called the chromosphere. It usually can be seen during solar eclipses as pink and red colors when the bright photosphere is obscured by the Moon, or through a filter on some strong spectral lines such as $\mathrm{H} \alpha$ or $\mathrm{Ca}$ II. The temperature in the 
photosphere falls to a minimum of $\sim 4,500 \mathrm{~K}$ until it rises again in the chromosphere, first relatively gently but then very rapidly in the transition region and finally reaches several million $\mathrm{K}$ in the corona. In most cases, the magnetized coronal plasma has a low plasma $\beta$, defined as the ratio between the thermal pressure and the magnetic pressure $\beta=\frac{P_{\text {ther }}}{P_{\text {mag }}}=\frac{n k_{B} T}{B^{2} /(8 \pi)}$, where $n, T$, and $B$ are respectively the plasma density, temperature and magnetic field strength. Under the condition of $\beta \ll 1$ the electrons and ions are fully coupled with the coronal magnetic field-the "frozen-in" effect. As a result, the distribution of hot coronal plasma traces out the structure of coronal magnetic field, in many cases, observed as coronal loops. Figure 1.2 summarizes the temperature and density as a function of height starting from the photosphere through the chromosphere, transition region to the low corona.

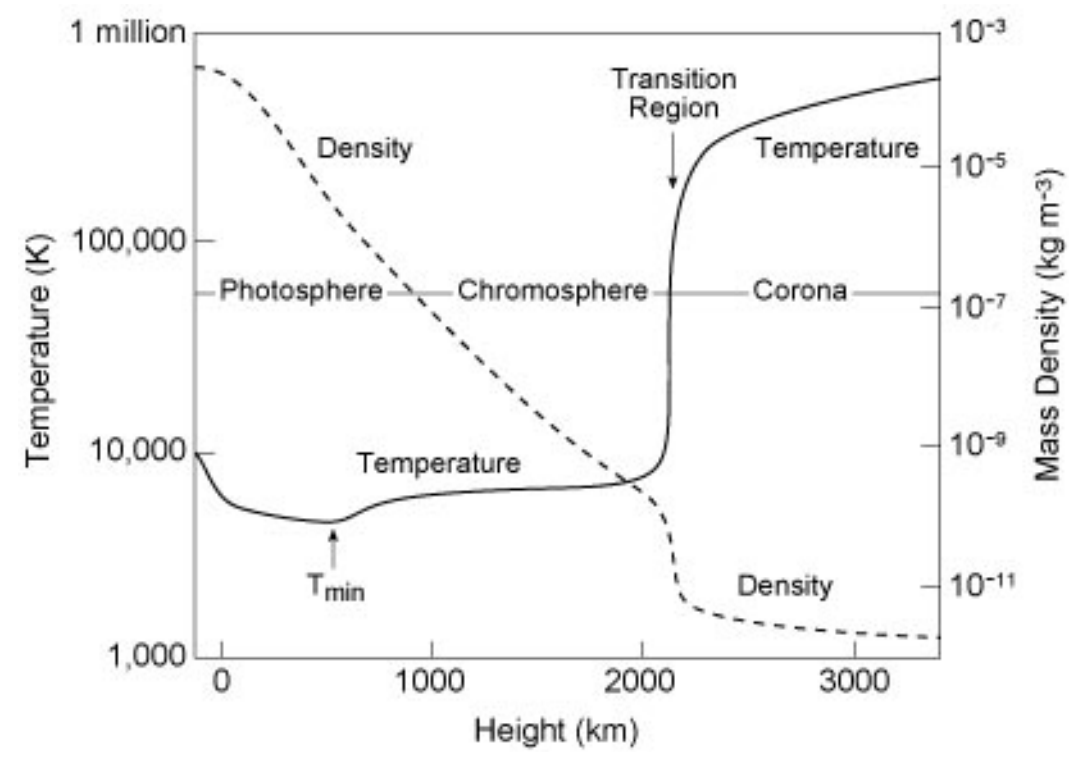

Fig. 1.2. - Temperature and density change as a function of height in the solar atmosphere, starting from the photosphere (by Dr. Eugene Avrett of SAO/CfA; reproduced with permission).

Due to the complexity of the coronal magnetic field and the low plasma $\beta$ environment, the corona is highly inhomogeneous and structured. On macroscopic scales 
there are regions called "coronal holes" where the plasma is cooler and less dense than the average quiet corona, and the magnetic field opens into interplanetary space (Figure 1.3(A)). High-speed solar wind is formed in coronal holes and propagates outward along the open field lines (see Cranmer 2002 for a review). Helmet-streamers are often seen near the equator which are organized by closed magnetic field loops (Figure 1.3(B)). In active regions - where most solar activities originate - coronal loops are the dominant structures (Figure 1.3(C)). These are loop-like structures connecting regions of opposite magnetic polarity in the photosphere, which are often loaded with over-dense plasma. Even a single loop seen in X-ray or extreme ultra-violet (EUV) images can be highly inhomogeneous in the transverse direction - it can be a bundle of very thin threads that are probably under-resolved by the current instrumentation (Figure 1.3(D)).

The Sun has, of course, a significant influence on the life on Earth. Its electromagnetic radiation sustains the life, drives the weather system, and provides most of the energy we are currently using. In addition to these everyday processes, the Sun can also influence us in a more violent fashion through solar activity - solar flares, coronal mass ejections (CMEs), co-rotating interaction regions, etc. A major solar flare is capable of releasing a large amount of energy, up to $10^{32}$ to $10^{33}$ ergs in a time scale of $10^{2}$ to $10^{3}$ seconds (Lin \& Hudson, 1976). Solar flares and associated CME events can strongly influence the Earth and near-Earth environment as a result of the intense EUV and X-ray radiation, energetic particle radiation, and the impact of coronal material on the magnetosphere. For example, an increased level of X-ray flux from solar flares can increase the ionization of Earth's upper atmosphere and disrupt radio communication; energetic particles and high-energy electromagnetic radiation (hard X-ray or $\gamma$-ray) can be damaging to spacecraft devices and astronauts' 

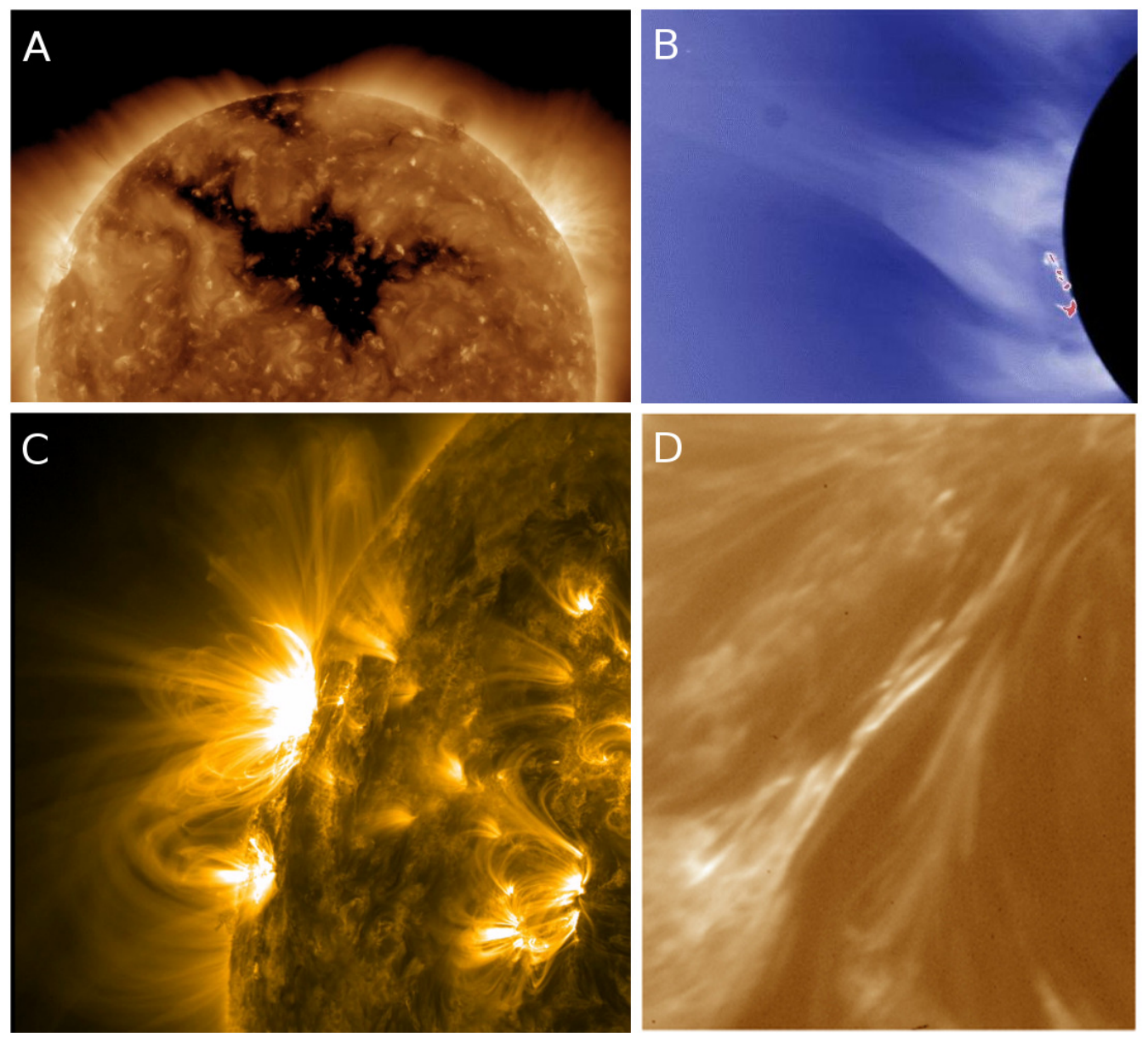

Fig. 1.3. - Structures of the solar corona. (A) A coronal hole (the dark region sprawling a large area of the Sun's surface) is a low-density region where the coronal magnetic field opens freely into interplanetary space, seen by the Solar Dynamics Observatory (SDO) in EUV band at $195 \AA$ on 2010 August 28 (image courtesy NASA). (B) Helmet streamers seen at the Sun's limb. They are large cap-like coronal structures organized by closed magnetic field lines. The pointed peaks are formed by blowing solar wind in the spaces between the streamers (image courtesy NASA). (C) A canopy of coronal loops from a pair of significant active regions observed by the SDO on 2013 January 6. The loops are heated plasma organized by the coronal magnetic field emanating from these active regions (image courtesy NASA). (D) Braided thin threads observed by the High-resolution Coronal Imager ( $\mathrm{Hi}-\mathrm{C}$ ) launched on a sounding rocket on 2012 July 11 with the highest spatial resolution to date $(\sim 150$ $\mathrm{km})$. The braided threads are thought to be undergoing reconnection and release energy into the corona (adapted by permission from Macmillan Publishers Ltd: Nature Cirtain et al., 2013, copyright 2013). 
health; direct radio emission from solar bursts can be a source of radio noise storm enough to interfere with cell phones and the reception of global positioning system signals; an Earth-directed CME can cause a geomagnetic storm that disrupts the Earth's magnetosphere and may even knock out electric power for extended periods of time. Collectively, these are referred as space weather phenomena. Nowadays, the increasing dependence of society on electronics, especially wireless electronics, means a growing risk of the society being exposed to the effects of space weather. Therefore, understanding in part the physical processes of the major space weather drivers is one of the primary motivations of this dissertation. My focus here is solar flares.

\subsection{Solar Flares}

\subsubsection{Overview}

A solar flare is a sudden and intense brightening over a localized region of the Sun's surface. Flares are the most powerful explosions in the solar system. They release a significant amount of energy over a short time scale. The first solar flare was observed by Richard C. Carrington and independently by Richard Hodgson on September 1, 1859 (Carrington, 1859). They saw a localized visible brightening within a sunspot group in white light, now known as a "white-light flare". Soon after Carrington and Hodgson's discovery, with the help of spectroscopy (after George E. Hale) the Sun was extensively observed in the $\mathrm{H} \alpha$ line, which, as has been mentioned before, originates in the chromosphere. From then on, reports of solar flares became much more frequent. The reason is that flares produce a strong $\mathrm{H} \alpha$ emission line that is easily detected against the background. Flare-associated radio emission was first detected in 1942 by military radar during World War II. It was found that the total radio 
intensity may increase by several orders of magnitude, which suggests that flares are not only thermal phenomena, but should involve production of non-thermal particles. In the late 1950s hard X-ray (HXR) observations become possible with balloons and rockets, and enhancements in HXR flux have been frequently observed during solar flares, which, also indicate the existence of non-thermal particles. Spectroscopic HXR observations by Brown (1971) led to the conclusion that the HXR-producing energetic particles contain a significant fraction of the total energy released in a flare. In the modern era, with the help of observations from space, solar flares have been observed across virtually the entire electromagnetic spectrum from radio waves to $\gamma$-rays, which has greatly advanced our knowledge of solar flares.

Solar flares are often classified in five categories-A, B, C, M, and X-based on their peak flux measured at the Earth in soft X-ray (SXR) wavelength from 1-8 (1.5 to $12.4 \mathrm{keV})$, as shown in Table 1.1 . Within a flare category there is a linear scale from 1 to 9.9 (except X-flares). For example, an M3 flare has a peak flux of $3 \times 10^{-5} \mathrm{~W} \mathrm{~m}^{-2}$, three times stronger than an M1 flare. X-class flares exceeding class X9 (peak flux $9 \times 10^{-4} \mathrm{~W} \mathrm{~m}^{-2}$ ) are occasionally observed. The largest flare measured with modern instruments exceeded X28, saturating the Geostationary Operational Environmental Satellite (GOES) detectors.

Table 1.1: Solar flare classifications

\begin{tabular}{cc}
\hline \hline Classification & $\begin{array}{c}\text { Peak flux from 1-8 } \\
\text { Watts } \mathrm{m}^{-2}\end{array}$ \\
\hline $\mathrm{A}$ & $<10^{-7}$ \\
$\mathrm{~B}$ & $10^{-7}-10^{-6}$ \\
$\mathrm{C}$ & $10^{-6}-10^{-5}$ \\
$\mathrm{M}$ & $10^{-5}-10^{-4}$ \\
$\mathrm{X}$ & $>10^{-4}$ \\
\hline \hline
\end{tabular}


Radiation from a solar flare often shows a variety of timing at different wavelengths, as shown schematically in Figure 1.4. A flare, in general, can be sub-divided into three phases: preflare phase, impulsive phase, and gradual phase (or decay phase). Flare-related activity is usually observed to commence prior to the major flare energy release in the preflare phase. For example, sometimes homologous and sympathetic flares can serve as precursors. Homologous flares are those located in the same active region with similar emission patterns, while sympathetic flares usually occur at different locations but erupt nearly synchronously. Characterized by rapid and intense emissions in radio, HXR and $\gamma$-ray wavelengths, the impulsive phase is the main phase of flare energy release and particle acceleration. In HXR wavelengths, the presence of a double HXR footpoint source at the chromospheric level is a characteristic of this phase (Hoyng et al., 1981; Sakao, 1994), although a single footpoint source or multiple component sources are also frequently seen (e.g., Temmer et al., 2007; Liu et al., 2007a). Incoherent centimeter radio emission usually outlines flaring loops occupied by non-thermal electrons. Intense radio bursts at decimeter and meter wavelengths, which are primarily due to coherent emission mechanisms, show a general but loose association with the impulsive energy release phase (Benz et al., 2005; White et al., 2011). In the gradual phase, the interplay between energy transport and atmosphere response becomes the dominant process. In most cases, bright postflare loops can be seen in EUV and SXR wavelengths due to plasma heating.

\subsubsection{The Standard Flare Model}

The energy released in solar flares is believed to have a magnetic origin. It is thought that the energy is previously stored in the coronal magnetic field as "free" magnetic energy - the excess energy contained in the magnetic field comparing to a potential 


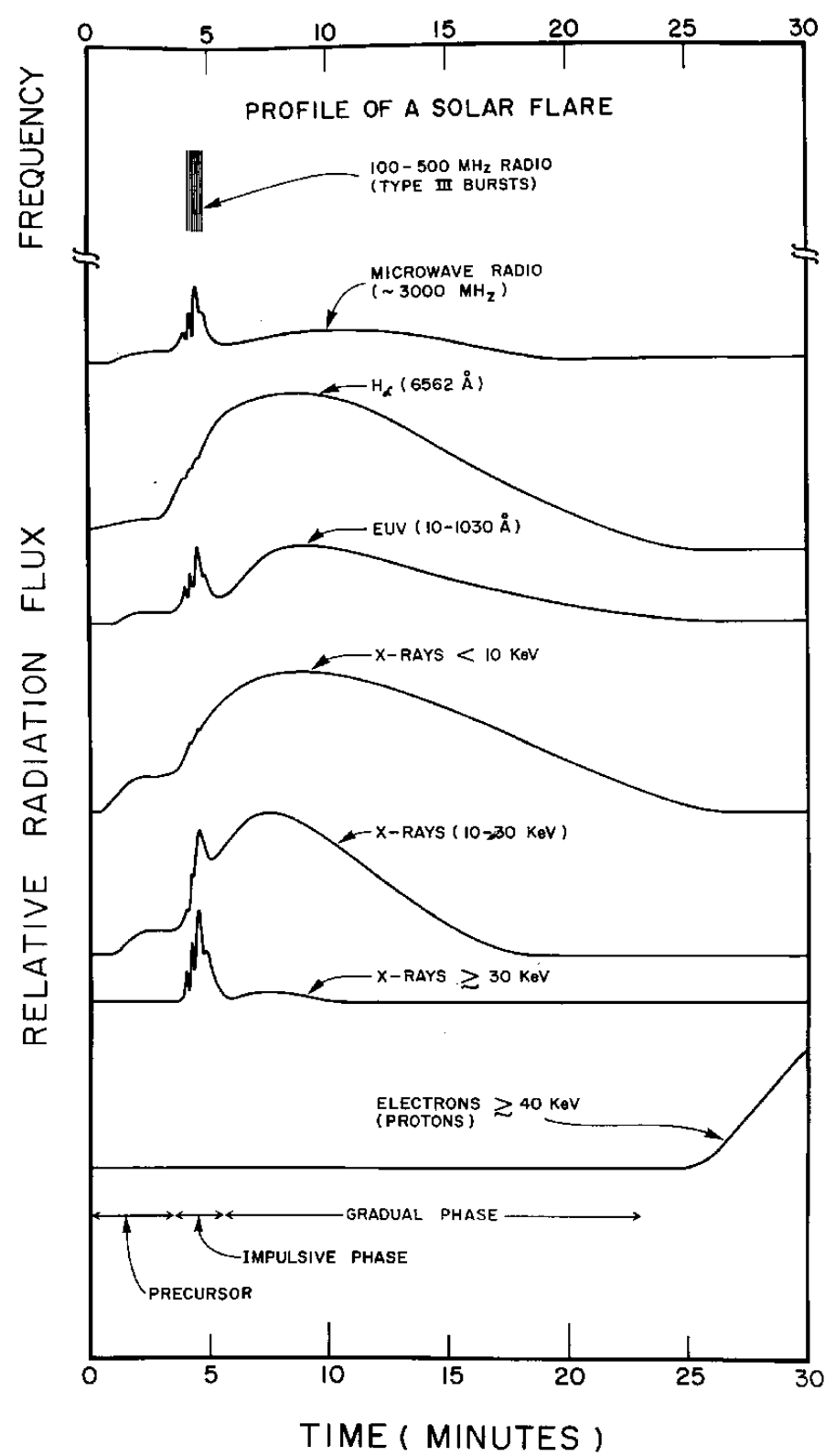

Fig. 1.4. - Schematic representation of different phases of a solar flare (from Kane, 1974). In general, a flare can be sub-divided into three different phases-preflare phase, impulsive phase, and gradual phase (or decay phase)-based on the different temporal behavior of flare radiation observed at different wavelengths.

state-resulting from photospheric or sub-photospheric motions. Photospheric magnetic field measurements indicate that the flaring region is usually highly sheared (Hagyard et al., 1990), either from an already sheared emerging magnetic flux (Geor- 
goulis \& LaBonte, 2006) or from slow footpoint motions that stretch and twist the magnetic loops in the active region (Krall et al., 1982; Brown et al., 2003). The large-scale sheared fields in active regions are able to store a large amount of free magnetic energy through steady motions. However, since the impulsive energy release process leaves few direct observational traces until the energy appears in the form of accelerated particles and very hot heated plasma, it is not fully understood how the magnetic energy is suddenly released and how this energy is rapidly converted into non-thermal energy in accelerated particles, thermal plasma heating and mass motion. A variety of models has been proposed to account for these processes, most of which involve a magnetic reconnection process, in which magnetic field lines change their topological connectivity and reconfigure to a lower-energy state (Miller et al., 1997; Zharkova et al., 2011).

Neupert (1968) found that the SXR flux corresponds to the time integral of incoherent centimeter radio flux since the start of a flare, referred to as the "Neupert Effect". As the incoherent centimeter radio emission results from relativistic electrons gyrating along the magnetic field lines in the corona, and the same population of electrons also produces HXR emission as they hit the dense chromosphere, it is not surprising that later a similar relation was found between the SXR flux and the time integral of the observed HXR flux. These observations suggest a strong causal relation between the SXR emission produced by heated plasma and the HXR/radio emission resulting from energetic electrons. Yet where the magnetic energy is released is still unclear. The first evidence came from Masuda et al. (1994), showing a third HXR source located above the SXR flaring loop (Figure 1.5(A)). There are also signatures of cusp-shaped SXR flaring arcades in long-duration flares suggesting magnetic reconnections above the SXR arcades (e.g., Tsuneta et al., 1992; Forbes \& 
Acton, 1996, see also Figure 1.5(B)). These observations suggest that the primary magnetic energy release in flares probably occurs in the low corona, and that there should be strong coupling between the corona and chromosphere during flares. This suggestion is also compatible with the time-of-flight analysis of footpoint HXR sources at different energies (Aschwanden et al., 1995b), for which low-energy HXR photons are produced by low-energy electrons that need a longer time than their high-energy counterparts to arrive at the chromosphere from the coronal energy release site.
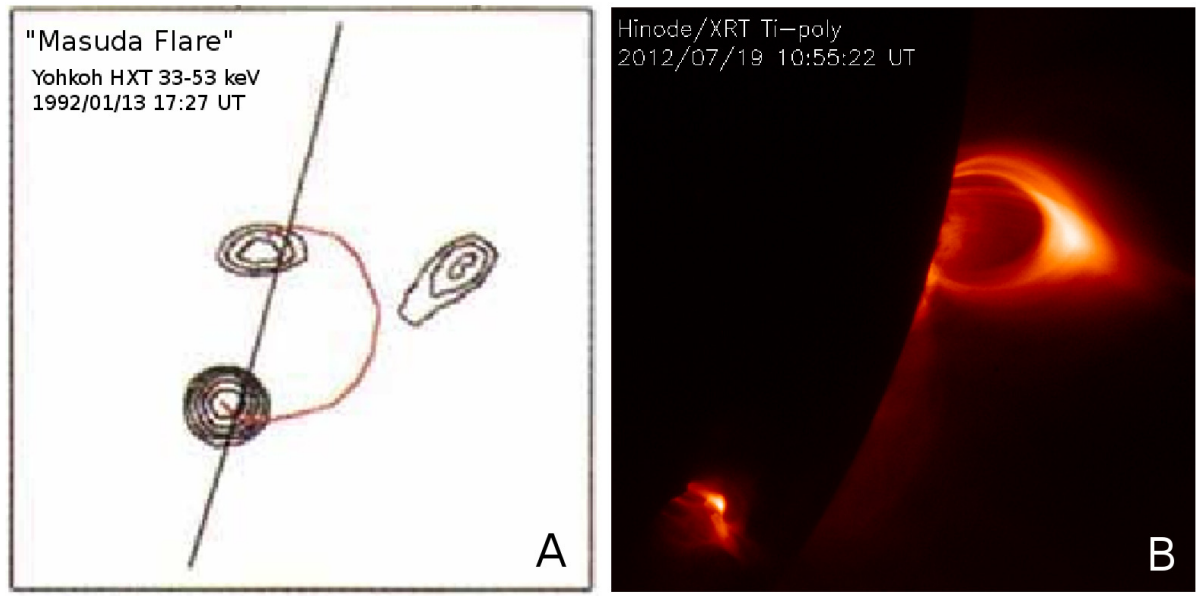

Fig. 1.5.- (A) The celebrated "Masuda Flare" showing two footpoint HXR sources as well as an additional coronal HXR source located above the SXR flaring loop (solid line); figure adapted by permission from Macmillan Publishers Ltd: Nature Masuda et al. (1994), copyright 1994. (B) A cusp-shaped arcade captured by the Xray Telescope (XRT) on board the Hinode satellite on 2012 July 19. These features are thought to indicate a magnetic reconnection site above the cusp, which involves magnetic energy release that heats and enhances the loops such that they emit the SXR emission seen by the XRT. Image courtesy of the Hinode/XRT team.

The observations described above have led to a simplified scenario: the standard flare model, or the CSHKP model, named after the contributors: Carmichael, Sturrock, Hirayama, Kopp and Pneuman (Carmichael, 1964; Sturrock, 1966; Hirayama, 1974; Kopp \& Pneuman, 1976). It assumes that magnetic reconnection occurs in the corona. Particles are postulated to be accelerated near the reconnection region by the 
released energy, yet the acceleration process is not part of the model. These particles, especially energetic electrons, can then precipitate along the magnetic field lines to the chromosphere as electron beams and deposit most of their energy in the cool, dense chromospheric plasma at the footpoints of the flare loop through collisional heating. The heated plasma then expands up into the loop and changes the properties of the loop plasma above the footpoints, termed "chromospheric evaporation" (Figure 1.6, from Magara et al., 1996). With this model, several observational phenomena during flares can be explained coherently, including the Neupert effect, the SXR flare loop connected by HXR footpoints, the cusp-shaped SXR arcades, the above-the-loop-top "Masuda" HXR source, the softer spectrum of the coronal HXR source than its footpoint counterpart, and the increasing emission measure of the SXR/EUV loop source during the impulsive phase.

Although the standard flare model can account for a variety of observed flare phenomena, deviations from the standard model have been frequently reported, and alternative models have been proposed. For example, in a significant fraction of events $(\sim 25 \%)$, there are strong deviations from the timing predicted by the Neupert effect (Dennis \& Zarro, 1993; McTiernan et al., 1999; Veronig et al., 2002); in some flare events, the coronal loops are so dense and collisionally thick to non-thermal electrons that they fail to reach the chromosphere (Veronig \& Brown, 2004); there is also a long-standing "number problem" associated with the standard model-the observed high intensity of the HXR, EUV, and optical continuum implies that the total number of energetic electrons required is comparable to that available in the coronal energy release region (e.g., Fletcher et al., 2007; Krucker et al., 2010)—which places strong requirements to the acceleration mechanism(s) and the associated supply and resupply problems. The concept of a "return current" may solve the problem for replenishing 


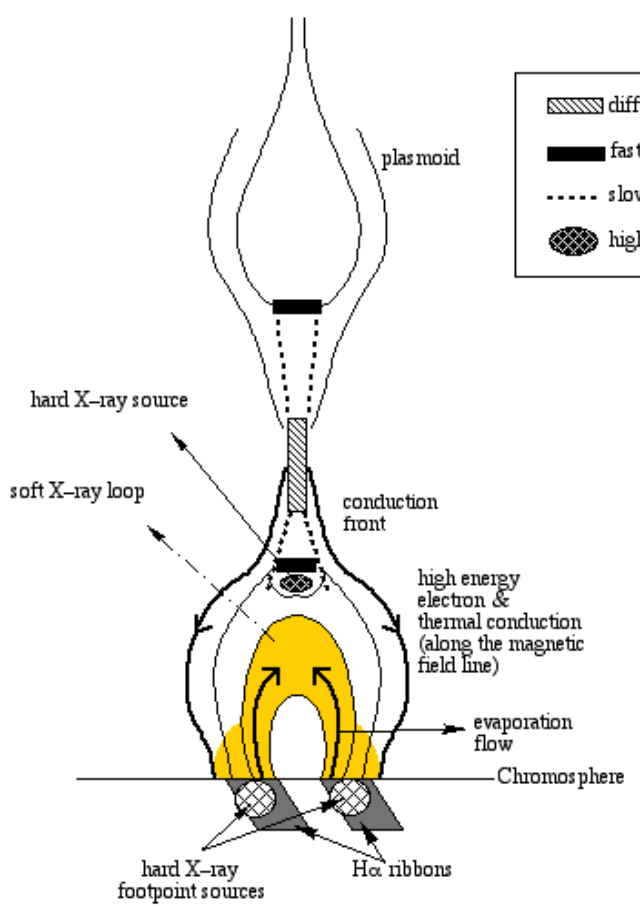

Impulsive flares (or impulsive phase)

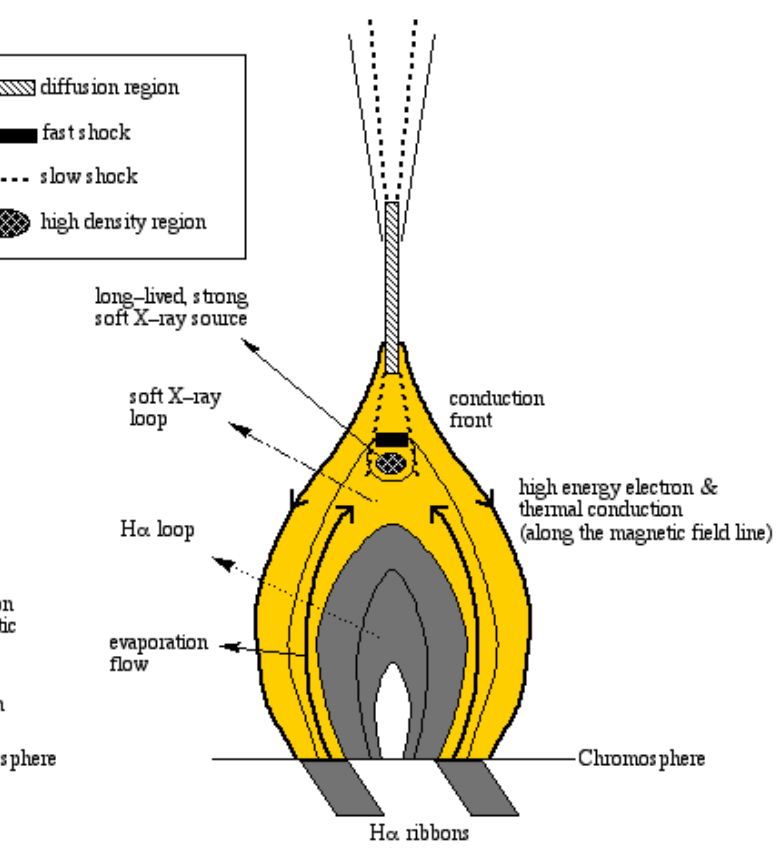

LDE flares (or gradual phase)

Fig. 1.6. - Schematic representations showing the standard flare model (CSHKP model) in accounting for observed features of solar flares in $\mathrm{H} \alpha$, SXR, and HXR wavelengths (from Magara et al., 1996, reproduced by permission of the AAS).

the acceleration region to conserve charge and current neutrality (e.g., Spicer \& Sudan, 1984; van den Oord, 1990). However, no direct observational signature of these return currents has been reported other than some indirect evidence (Battaglia \& Benz, 2008). There have been other varieties of flare models proposed, including those with different flare geometries (e.g., Heyvaerts et al., 1977; Aulanier et al., 2000), those that do not require chromospheric evaporation (Acton et al., 1992; Dennis \& Zarro, 1993), and those in which the energy is not transported downward by electron beams but by large-scale Alfvèn waves (Fletcher \& Hudson, 2008).

From the discussions above, it is clear that multi-wavelength observations are essential in disentangling the complexities of the various physical processes in solar 
flares. This dissertation takes advantage of state-of-art solar instrumentation for multi-wavelength observations as detailed in Section 1.3. In particular, I focus on studies in radio and X-ray wavelengths since the emissions sample most sources of energetic electrons produced by flares. The next two sections are devoted to reviewing the relevant radio and X-ray emission mechanisms in flares and summarizing their diagnostic uses.

\subsubsection{Radio Emission}

Radio emission from solar flares provides a number of unique means of addressing some of the long-standing questions in the physics of solar flares. It has played an important role in shaping our understanding of flare physics for more than half a century. For example, as mentioned before, the Neupert effect was first discovered through the correlation between the microwave radio flux and the SXR flux (Neupert, 1968), which was one of the key components leading to the establishment of the standard flare model. A number of distinct emission mechanisms can be responsible for producing radiation at different radio wavelengths, both incoherent and coherent, providing rich diagnostic potential of the physical processes in flares.

In radio astronomy, measurements are usually described in terms of flux density $S_{\nu}$

$$
S_{\nu}=\int_{\Omega} I_{\nu} \cos \theta d \Omega
$$

where $I_{\nu}$ is the specific intensity defined as

$$
I_{\nu}=\frac{d P}{\cos \theta d \sigma d \nu d \Omega}
$$

where $P$ is the received power, $\nu, \sigma, \Omega$, and $\theta$ are radio frequency, surface area, 
source solid angle, and the angle of the source away from the receiving surface normal, respectively. For sources with angular size $\ll 1 \mathrm{rad}, \cos \theta \approx 1$, then the expression for flux density is simplified to $S_{\nu}=\int_{\Omega} I_{\nu} d \Omega$.

For blackbody radiation, at radio frequencies $h \nu \ll k_{B} T$, the Rayleigh-Jeans approximation is valid and the Planck function can be simplified to

$$
I_{\nu}=\frac{2 k_{B} T \nu^{2}}{c^{2}} .
$$

It is convenient to define a brightness temperature

$$
T_{b}=\frac{I_{\nu} c^{2}}{2 k_{B} \nu^{2}}
$$

and the flux density $S_{\nu}$ is then

$$
S_{\nu}=\frac{2 k_{B} \nu^{2}}{c^{2}} \int_{\Omega} T_{b} d \Omega
$$

irrespective of the origin of the radiation based on the observed specific intensity $I_{\nu}$ or flux density $S_{\nu}$. For radio imaging instruments, observations are often limited to an area of the sky within which the antenna can receive a response, referred as the beam of the radio telescope with a solid angle $\Omega_{\mathrm{bm}}$. A compact source and an extended source with an angular size exceeding the beam size $\Omega_{\mathrm{bm}}$ are different in terms of the limit of the integral in Equation 1.5: the former is an integration over the source solid angle, while the latter is an integration over the beam size. For an optically thick source with an effective temperature $T_{\text {eff }}=\langle E\rangle / k_{B}$ (where $\langle E\rangle$ is the mean energy of the emitting electrons in the source) and incoherent emission, $T_{b}=T_{\text {eff }}$. For an optically thin source, $T_{b} \approx \tau_{\nu} T_{\text {eff }}$, where $\tau_{\nu}$ is the optical depth. For 
coherent emission, the observed brightness temperature $T_{b}$ can be much higher than the effective temperature of the source $T_{\text {eff }}$.

In the magnetized plasma of the solar atmosphere, two characteristic frequencies are important in solar radio emission: the electron plasma frequency $\nu_{\mathrm{pe}}=$ $\omega_{\mathrm{pe}} /(2 \pi)=\sqrt{n_{e} e^{2} /\left(\pi m_{e}\right)} \approx 8980 \sqrt{n_{e}} \mathrm{~Hz}$, and the electron gyrofrequency $\nu_{\mathrm{ce}}=$ $\omega_{\text {ce }} /(2 \pi)=e B /\left(2 \pi m_{e} c\right) \approx 2.8 \times 10^{6} B \mathrm{~Hz}$, where $n_{e}$ is the electron density, $e$ and $m_{e}$ are the electron charge and mass, and $B$ is the magnetic field strength. Solar radio radiation is related to emission at a number of natural plasma wave modes. These wave modes can generally be grouped as electromagnetic and electrostatic according to whether or not there is an oscillating magnetic field component (there is, of course, a third category - magnetohydrodynamic waves, which are not directly related to radio emission). Electromagnetic waves can propagate in the plasma medium and be observed as electromagnetic radiation. In contrast, electrostatic waves only oscillate locally. They need to be converted to electromagnetic waves through secondary wave-wave or wave-particle process before they can escape and be recorded by a radio telescope. Table 1.2 summarizes the plasma wave modes that are related to electron oscillations, categorized by their electromagnetic characters as well as propagating conditions (only the cases of parallel and perpendicular propagation are shown).

A number of emission mechanisms, both coherent and incoherent, can result in observable radio emission in solar flares. The dominant mechanism during flares depends on both the wavelengths observed and the plasma conditions in the source. Generally, in a flaring region, thermal bremsstrahlung emission and absorption are ubiquitous and act as a thermal "background". Gyrosynchrontron radiation dominates the emission at centimeter and millimeter wavelengths $(\mathrm{cm}-\lambda$ and $\mathrm{mm}-\lambda)$. In the meter and decimeter wavelengths $(\mathrm{m}-\lambda$ and $\mathrm{dm}-\lambda)$, coherent radiation often be- 
Table 1.2: Waves in a magnetized plasma

\begin{tabular}{l|c|c|c}
\hline \hline EM character & Condition & Dispersion Relation & Name \\
\hline \multirow{2}{*}{ Electromagnetic } & $\vec{k} \perp \overrightarrow{B_{0}}$ & $\frac{c^{2} k^{2}}{\omega^{2}}=1-\frac{\omega_{\mathrm{pe}}^{2}}{\omega^{2}}$ & o mode \\
& $\vec{k} \perp \overrightarrow{B_{0}}$ & $\frac{c^{2} k^{2}}{\omega^{2}}=1-\frac{\omega_{\mathrm{pe}}^{2}}{\omega^{2}} \frac{\omega_{\mathrm{pe}}^{2}}{\omega^{2}-\omega_{\mathrm{uh}}^{2}}$ & $\mathrm{x}$ mode $^{1}$ \\
& $\vec{k} \| \overrightarrow{B_{0}}$ & $\frac{c^{2} k^{2}}{\omega^{2}}=1-\frac{\omega_{\mathrm{pe}}^{2} / \omega^{2}}{1+\omega_{\mathrm{ce}} / \omega}$ & $\mathrm{L}$ mode \\
& $\vec{k} \| \overrightarrow{B_{0}}$ & $\frac{c^{2} k^{2}}{\omega^{2}}=1-\frac{\omega_{\mathrm{pe}}^{2} / \omega^{2}}{1-\omega_{\mathrm{ce}} / \omega}$ & $\mathrm{R}$ mode ${ }^{2}$ \\
\hline Electrostatic & $\vec{k} \| \overrightarrow{B_{0}}$ & $\omega^{2}=\omega_{\mathrm{pe}}^{2}+3 k^{2} v_{\mathrm{th}}^{2}$ & Langmuir waves \\
\hline \hline
\end{tabular}

Note: $k$-wave number, $B_{0}$ - background magnetic field, $\omega$ - wave frequency, $\omega_{\mathrm{uh}}-$ upper-hybrid frequency, $v_{\mathrm{th}}=\sqrt{k_{B} T / m_{e}}$ - electron thermal velocity in a plasma of temperature $T$.

1 The low frequency branch $\left(\omega<\omega_{\mathrm{uh}}\right)$ of the $\mathrm{x}$ mode is referred as "z mode".

2 The low frequency branch $\left(\omega<\omega_{\text {ce }}\right)$ of the $\mathrm{R}$ mode is referred as "whistler mode".

comes the dominant emission mechanism (c.f., Figure 4.1 in Gary \& Hurford, 2004). This section summarizes these emission mechanisms and how they are manifested in solar radio emission. More detailed reviews can be found in Dulk (1985) and Bastian et al. (1998).

\section{Thermal Bremsstrahlung Radiation}

The upper solar atmosphere mainly consists of fully-ionized plasma, i.e., electrons and ions. "Braking radiation" from incident electrons as they are deflected by the Coulomb field of a quasi-stationary (much heavier) ion results in bremsstrahlung emission (or free-free emission). The emitted photon energy $\epsilon$ is proportional to the energy change $\Delta E_{e}$ of the incident electron after the deflection. In radio wavelengths, the photons are produced by weak interactions, meaning the energy change $\Delta E_{e}$ is 
much smaller than the initial kinetic energy of the incident electron $E_{e}$.

For a thermal plasma of temperature $T$ with a Maxwellian-Boltzmann distribution

$$
f_{e}(E)=\frac{2 n_{e}}{\sqrt{\pi}\left(k_{B} T^{3 / 2}\right)} E^{1 / 2} \exp \left(-\frac{E}{k_{B} T}\right), \text { electrons } \mathrm{cm}^{-3} \mathrm{erg}^{-1}
$$

the bremsstrahlung absorption coefficient can be derived to be (Dulk, 1985)

$$
\begin{aligned}
\kappa_{\nu} \approx & \sum_{i} \frac{1}{3 c}\left(\frac{2}{\pi}\right)^{1 / 2} \frac{\nu_{\mathrm{pe}}^{2}}{\nu^{2}} \frac{4 \pi Z_{i}^{2} n_{i} e^{4}}{m_{e}^{1 / 2}\left(k_{B} T\right)^{3 / 2}} \frac{\pi}{\sqrt{3}} G(T, \nu) \\
\approx & 9.78 \times 10^{-3} \frac{n_{e}}{\nu^{2} T^{3 / 2}} \sum_{i} Z_{i}^{2} n_{i} \\
& \times \begin{cases}18.2+\ln T^{3 / 2}-\ln \nu & \left(T<2 \times 10^{5} K\right) \\
24.5+\ln T-\ln \nu & \left(T>2 \times 10^{5} K\right),\end{cases}
\end{aligned}
$$

where $n_{e}, n_{i}$ are the plasma electron and ion density, $Z_{i}$ and $n_{i}$ are the ion charge and number density, $G(T, \nu)$ is the Gaunt factor proportional to $\ln \left(b_{\max } / b_{\min }\right)$ (where $b_{\max }$ and $b_{\min }$ are the maximum and minimum values of impact parameter $b$ ). A simplified expression of the absorption effect is often used

$$
\kappa_{\nu}=\frac{\zeta n_{e}^{2}}{\nu^{2} T^{3 / 2}},
$$

in which a coefficient $\zeta$ that includes the logarithmic terms in Equation 1.7 is introduced. In most cases, $\zeta$ only slightly depends on plasma parameters in the source and can be approximated as a constant. The bremsstrahlung emissivity $\eta_{\nu}$ is related to $\kappa_{\nu}$ by Kirchhoff's Law

$$
\eta_{\nu}=\frac{\kappa_{\nu} k_{B} T \nu^{2}}{c^{2}}
$$

In the solar atmosphere, the corona has an appreciable optical thickness due to the 
bremsstrahlung emission mechanism at low frequencies, and becomes optically thin at high frequencies. The chromosphere, because of its high density and low temperature, remains optically thick to the highest radio frequencies.

Figure 1.7(A) (from Gary \& Hurford, 2004) shows the bremsstrahlung brightness temperature spectrum from an isothermal and homogeneous corona at $T_{\text {cor }}=10^{6}$ $\mathrm{K}$ overlying an isothermal and homogeneous chromosphere at $T_{\mathrm{chr}}=10^{4} \mathrm{~K}$. The arrows shown on the plot indicate how the spectrum is shifted by increasing the coronal temperature $T$ and column emission measure $n_{e}^{2} L$ by a factor of four, without changing the spectral shape. Using this spectral feature, coronal temperature and density in an active region can be derived based on radio spectral observations (Gary \& Hurford, 1994).

Another diagnostic available using radio emission due to thermal bremsstrahlung is from polarization measurements. As the solar corona is magnetized, the presence of a magnetic field $B$ can result in different absorption coefficients for the $o$ - and $x$-modes, and the absorption coefficient becomes

$$
\kappa_{\nu}^{o, x}=\frac{\zeta n_{e}^{2}}{\left(\nu \pm \nu_{\mathrm{ce}}|\cos \theta|\right)^{2} T^{3 / 2}}
$$

where $\theta$ is the angle between the magnetic field and the wave normal direction. In most cases, the $o$ - and $x$-modes correspond to two orthogonal circular polarizationsleft-handed and right-handed circular polarizations (LCP \& RCP). Because the measured radio brightness temperature is sensitive to the absorption coefficient $\kappa_{\nu}$ (for the optically thin case $T_{b} \propto \tau_{\nu} \propto \kappa_{\nu}$; for the optically thick case, $T_{b} \approx T_{\text {eff }}(l)$ is the effective temperature at a height where the optical depth $\tau_{\nu}=\int \kappa_{\nu} d l$ is close to unity), $T_{b}$ is usually different at LCP and RCP, characterized by the parameter of the 

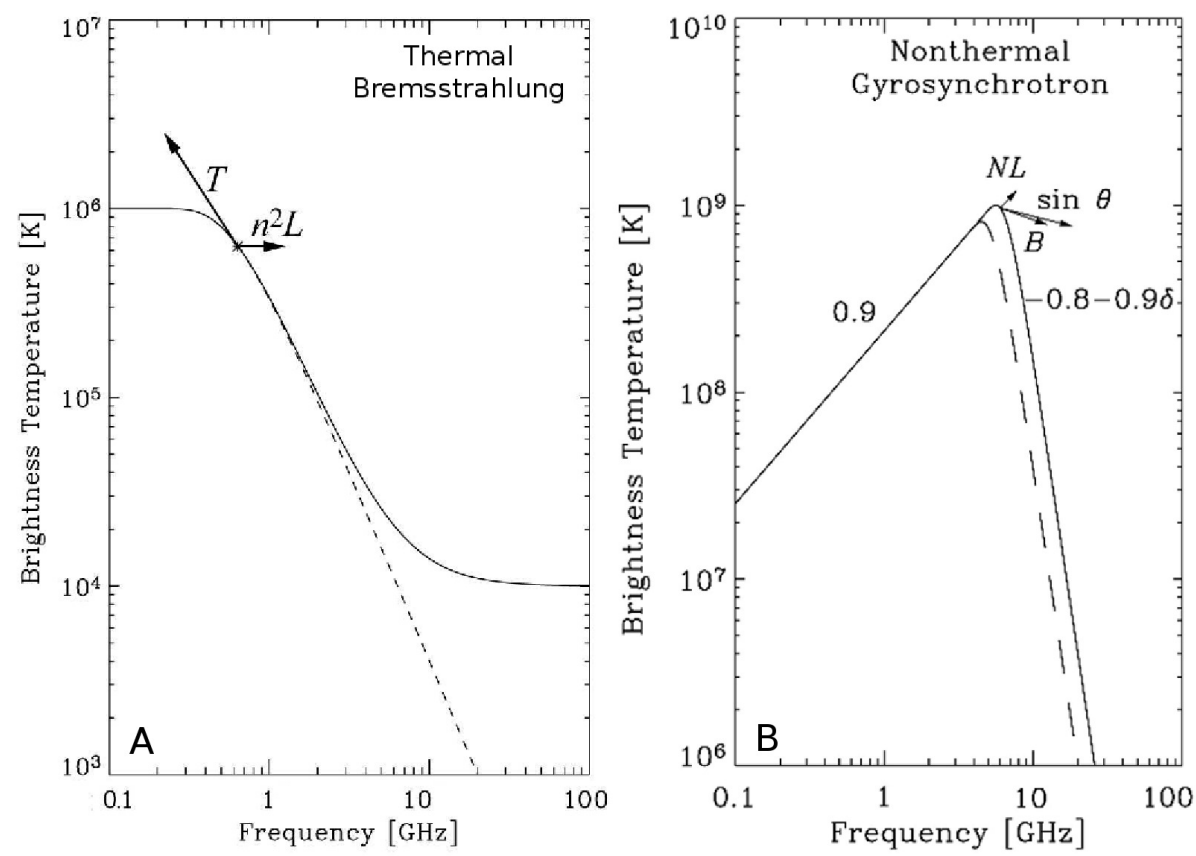

Fig. 1.7.- (A): A typical thermal bremmstrahlung brightness temperature spectrum. The solid line shows the spectrum from an isothermal corona at $10^{6} \mathrm{~K}$ overlying an isothermal chromosphere at $10^{4} \mathrm{~K}$. The dashed line shows the background-subtracted spectrum with the coronal contribution alone. (B): A typical non-thermal gyrosynchrotron brightness temperature spectrum from a homogeneous source with a powerlaw electron energy distribution. The solid and dashed lines show the $x$-mode and $o$-mode spectra, respectively. The arrows indicate the direction and magnitude of shift of the spectra for increases in the labelled parameters by a factor of four (from Gary \& Hurford, 2004).

degree of polarization $P$

$$
P=\frac{T_{b, R}-T_{b, L}}{T_{b, R}+T_{b, L}} .
$$

Under the weak magnetic field approximation, the equation of radiative transfer gives

$$
P \approx n \frac{\nu_{\mathrm{ce}}}{\nu} \cos \theta=n \frac{2.8 \times 10^{6}}{\nu} B_{l}
$$

where $B_{l}=B \cos \theta$ is the longitudinal component of the magnetic field, $n \equiv-\frac{d\left(\log T_{b}\right)}{d(\log \nu)}$ is the spectral index of the brightness temperature spectrum. For the case of optically 
thin source, $n=2$ since the brightness temperature is proportional to the thermal bremsstrahlung optical depth $T_{b} \propto \tau_{\nu}=\int \kappa_{\nu} d l \propto \nu^{-2}$. For the optically thick case, $n$ is determined by the temperature gradient with height in the source. As both the parameters of $n$ and $P$ are observables from spectropolarimetric radio observations, the radio data can be used to deduce the longitudinal magnetic field strength $B_{l}$ in the chromosphere and corona, as demonstrated by Grebinskij et al. (2000).

\section{Gyrosynchrotron Radiation}

The dominant emission mechanism during solar flares at frequencies $>3 \mathrm{GHz}$ is gyrosynchrotron emission, produced by mildly-relativistic electrons - with a Lorentz factor $1 \lesssim \gamma \lesssim 5$, energies of $10 \mathrm{~s} \mathrm{keV}$ to a few MeV—gyrating along magnetic field lines. The expressions for gyrosynchrotron emissivity and absorption are complex (Ramaty, 1969) and are often solved numerically with modern computing resources (an efficient numerical method can be found in Fleishman \& Kuznetsov, 2010), although simplified approximations have also been used (Petrosian, 1981; Dulk \& Marsh, 1982; Klein, 1987).

A typical spectrum of non-thermal gyrosynchrotron emission from a homogeneous source is shown in Figure 1.7(B), with the arrows indicating the direction and magnitude of shift for increases in the labelled parameters by a factor of four. The lowfrequency, rising, part of the spectrum is due to self-absorption. The positive slope is caused by the increasing effective energy of the non-thermal electrons producing the optically thick emission. The high frequency part of the spectrum is optically thin emission. The negative slope $\alpha$ is directly related to the power-law index of the parent electron energy distribution $\delta$, approximated by $\alpha \approx-0.8-0.9 \delta$ (Dulk \& Marsh, 1982). The gyrosynchrotron brightness temperature reaches a maximum 
value at the peak frequency $\nu_{\text {peak }}$, at which the optical depth $\tau_{\text {gyro }} \approx 1$.

Similar to X-ray radiation, gyrosynchrotron radiation is sensitive to flare accelerated energetic electrons, and provides a powerful diagnostic tool for inferring the electron energy distribution and its temporal evolution. In addition, gyrosynchrotron radiation is also very sensitive to the magnetic field strength and orientation, and therefore can be used to constrain the magnetic field in the flaring source. However, several effects such as Razin suppression (Klein, 1987) and anisotropy of the electron distribution (Fleishman \& Melnikov, 2003) can result in significant modification of the simple gyrosynchrotron spectrum shown in Figure 1.7(B). Reliable diagnostics thus would require high-quality spatially resolved spectropolarimetry data augmented by sophisticated modeling and forward-fitting methods. Although the feasibility of this approach has been previously demonstrated (Bastian et al., 1998; Bastian, 2006; Fleishman et al., 2009; Gary et al., 2013), such imaging spectropolarimetry data have not yet been routinely available.

\section{Coherent Radio Emissions}

Coherent radio emissions involve very efficient conversion of electron energy into some natural wave mode of plasma for electromagnetic radiation. The coherent process(es) can amplify the intensity of certain wave modes to very high levels in short time scales. As a result, an observed high brightness temperature is usually used as a characteristic feature to distinguish coherent from incoherent emission. However, in contrast to the incoherent radiation discussed above, the brightness temperature of coherent radio emissions is very difficult to interpret because it depends on the electron energy distribution in a non-linear fashion. Nevertheless, because coherent emissions are often emitted at or near some characteristic frequency in the plasma, such as 
the local electron plasma frequency $\nu_{\text {pe }}$, the electron gyrofrequency $\nu_{\text {ce }}$, and their harmonics and sometimes their combinations, their emitting frequency, polarization characteristic, and temporal variation can be used as powerful diagnostic tools for the plasma parameters of the source.

Solar radio bursts associated with flares at $\mathrm{m}-\lambda$ and $\mathrm{cm}-\lambda$ are believed to be dominated by coherent radio emissions. They are often observed to have a high brightness temperature and show a variety of features in the dynamic spectra-records of radio intensity as a function of time and frequency. Certain features are thought to be produced by different physical processes, and have been historically named as Type I, II, III, IV, and V bursts (Wild \& McCready, 1950; Dulk, 1985). Figure 1.8(A) illustrates the typical structures of various types of solar radio bursts in a dynamic spectrum. Type I bursts (or storms) are the most commonly observed radio burst phenomena of the Sun. The bursts, each lasting about a second, are usually highly polarized and appear in a cluster persisting for a long duration of anything from a few hours to days and weeks. They are believed to arise in large-scale closed-field regions. Type II bursts are characterized by a moderate frequency drift as a function of time $(d f / d t)$ in the dynamic spectrum. They are thought to be associated with travelling shocks in the corona. In contrast, the frequency drift rate of type III bursts is much greater. Hence they are believed to be produced by propagating fast electron beams with a speed of $\gtrsim 0.1 c$. Type IV bursts and decimetric continuum are usually characterized to be broadband and quasi-stable structures in the dynamic spectrum. They are sometimes superposed by or associated with a variety of fine structures, such as zebra-pattern bursts (Figure 1.8(B)), spike bursts (Figure 1.8(C)), fibers, pulsations, etc. Current theories indicate that they are from electrons trapped in magnetic mirrors or plasmoids. Type $\mathrm{V}$ bursts are continuum features seen to follow 
type III bursts or bursts groups and are thought to be caused by the same population of electrons producing the type III bursts.
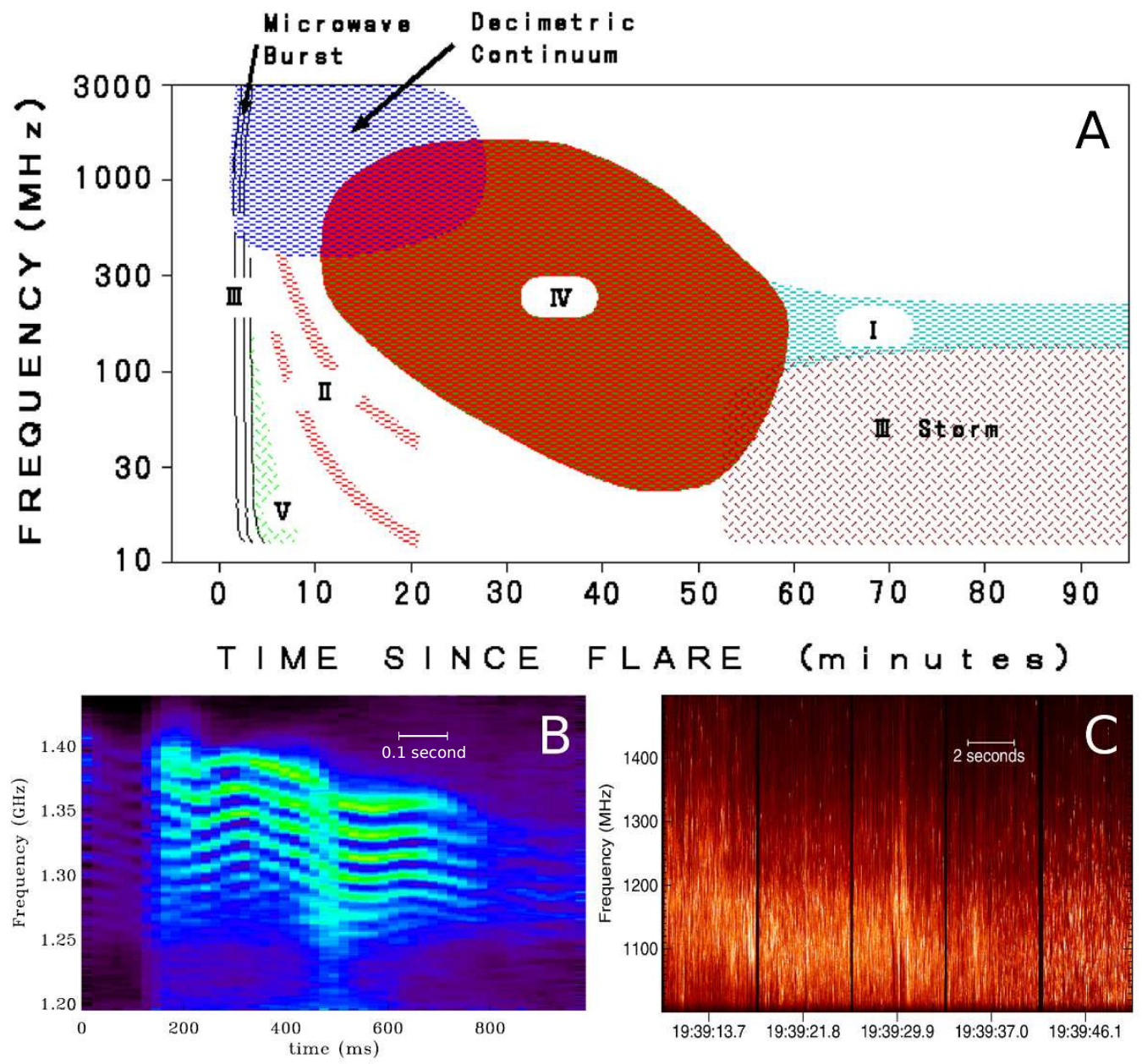

Fig. 1.8.- (A): Classification of solar radio bursts (credit HiRAS Solar Observatory). (B) Example of a decimetric zebra-pattern burst observed on 2006 December 14 obtained by the FST (from Chen et al., 2011). (C) Example of a spike burst observed on 2006 December 6 obtained by the FST (from Cliver et al., 2011).

Two coherent emission mechanisms are considered to be relevant for these solar radio bursts: plasma radiation and electron cyclotron maser radiation. There are of course other coherent emission mechanisms in the astrophysical context, such as those in pulsar radio emission (e.g., Hankins \& Eilek, 2007) which involve exotic processes and are inadequately understood so are not discussed here. 
PLASMA RADIATION: Plasma radiation often occurs at the plasma frequency $\nu_{\mathrm{pe}}$ and/or its harmonic $2 \nu_{\mathrm{pe}}$. As the plasma frequency depends only on the square root of the plasma density $\nu_{\mathrm{pe}} \propto \sqrt{n_{e}}$, and since the plasma density generally decreases with height in the solar corona, plasma radiation at higher frequencies usually originates from lower coronal heights and vice versa. In the high corona, it is likely that the plasma frequency $\nu_{\mathrm{pe}}$ is much greater than the electron gyrofrequency $\nu_{\mathrm{ce}}$, and usually the electrons are not energetic enough to emit at high harmonics of $\nu_{\mathrm{ce}}$, so that plasma radiation is favored at low radio frequencies (in the meter or decimeter wavelength range). At higher radio frequencies $(\gtrsim 3 \mathrm{GHz})$ plasma radiation becomes increasingly rare. The reason is that radiation close to the plasma frequency experiences increasingly strong absorption due to free-free absorption at higher frequencies, because $n_{e} \propto \nu_{\mathrm{pe}}^{2} \sim \nu^{2}$ such that the absorption coefficient in Equation 1.8 becomes $\kappa_{\nu} \propto n_{e}^{2} \nu^{-2} \propto \nu^{2}$

The plasma radiation mechanism involves complex and non-linear processes. Two major stages are necessary to produce the radiation: (a) production of a spectrum of Langmuir waves in the source, and (b) conversion of the longitudinal Langmuir waves into transverse (electromagnetic) waves to escape the source and be recorded by a radio telescope. The basic ideas of the emission theory were introduced by Ginzburg \& Zheleznyakov (1958), and a detailed discussion can be found in Dulk (1985). An outstanding example of a phenomenon attributed to plasma radiation is the type III radio burst, which arise from a propagating beam of energetic electrons in the corona. As the electrons propagate along magnetic field lines, a "bump-on-tail" instability can form and cause Langmuir waves to grow exponentially. Subsequent wave conversions into other wave modes can on one hand result in observable electromagnetic radiation, and on the other hand, limit the growth of the Langmuir waves (Bastian et al., 1998; 
Benz, 2002). As the beam propagates in the corona, at any given time the emission is generated near the local $\nu_{\mathrm{pe}}$ or $2 \nu_{\mathrm{pe}}$, which is a function of the instantaneous coronal height the beam has reached. As a result, in the dynamic spectrum, the frequency at which the highest intensity is located drifts rapidly with time, i.e. the frequency drift rate $d f / d t$ has a large value. Type II radio bursts are also considered to have an origin of plasma radiation. Their much slower frequency drifts indicate that these bursts are associated with travelling shocks in the corona. It is suggested that shock accelerated electrons can develop anisotropy that is unstable to the production of Langmuir waves, some of which are converted into electromagnetic waves and are observed (Wild \& Smerd, 1972; Bale et al., 1999; Schmidt \& Cairns, 2012).

ELECTRON CYCLOTRON MASERS: Maser stands for "Microwave Amplification by Stimulated Emission of Radiation". A maser was originally developed in laboratories as a device producing enhanced microwave emission. In the astrophysical context, the term has been used to describe phenomena of maser-like stimulated emission. Electron cyclotron maser $(\mathrm{ECM})$ radiation is thought to be the relevant emission mechanism for certain radio emissions observed from our Earth, other planets, the Sun, flare stars, to Blazar jets (see Treumann 2006 for a review). On the Sun, ECM is expected to operate in a magnetic trap, presumably in convergent magnetic fields in coronal loops, where electrons moving along the field lines escape the trap while those remaining in the trap form a loss-cone type instability (Melrose \& Dulk, 1982; Winglee \& Dulk, 1986; Kuznetsov \& Tsap, 2007) or a ring/shell type instability (Treumann, 2006) and emit radio emission.

Two types of ECM radiation are thought to be responsible for certain solar radio emissions. One considers the source condition of $\nu_{\text {pe }} / \nu_{\text {ce }} \ll 1$ (Holman et al., 1980; Melrose \& Dulk, 1982; Treumann, 2006), for which $x$-mode or $o$-mode waves 
are greatly enhanced near the fundamental or low harmonics of the electron gyrofrequency $\nu_{\text {ce }}$. Another considers $\nu_{\text {pe }} / \nu_{\text {ce }} \gg 1$ (Winglee \& Dulk, 1986; Kuznetsov \& Tsap, 2007), for which enhanced upper-hybrid waves are considered to be relevant and the emission frequency is near $\nu_{\mathrm{uh}}$ or its harmonic. The former is the most favorable process responsible for producing clusters of short duration (milliseconds) and narrowband $(\sim 10 \mathrm{MHz})$ bursts with very high brightness temperature $\left(\gtrsim 10^{12}\right.$ K) - the so-called "spike bursts" (Figure 1.8(C)). The latter is thought, by some authors, to be responsible for the type IV continuum bursts and zebra-pattern bursts, which appear in a dynamic spectrum as several parallel stripes separated regularly in frequency (Figure 1.8(B)), a study of which will be presented in Chapter 2.

Solar coherent radio emissions have been used for decades as a diagnostic tool in solar physics, such as to study flare magnetic energy release (Benz et al., 1996; Aschwanden \& Benz, 1997), to derive properties of the exciters such as shocks from type II bursts and electron beams from type III bursts (Wild et al., 1954; Mann et al., 1999; Chen \& Yan, 2008), to diagnose the plasma density and magnetic field strength in the source region (Aschwanden \& Benz, 1997; Zlotnik et al., 2003), and to measure the coronal-interplanetary density structure (Leblanc et al., 1998). However, the majority of the previous work has proceeded along two largely orthogonal paths: imaging and dynamic spectroscopy. Imaging observations have been provided by interferometers at a few discrete frequencies. Dynamic spectroscopic observations have been performed by spatially-integrated (total-power) radio spectrometers. A barrier of exploiting the coherent radio bursts has been a lack of instrumentation to do simultaneous imaging and dynamic spectroscopic observations. Such studies appear occasionally from combined observations by a interferometer and a spectrometer, and have already yielded significant insights such as in confirming the emission scenario of the bursts 
(Aschwanden et al., 1992; Altyntsev et al., 2005; Bain et al., 2012) and their relation to flare energy release (Paesold et al., 2001; Battaglia \& Benz, 2009). However, in order to exploit fully the diagnostic potential of these bursts, an instrument capable of doing both imaging and dynamic spectroscopy over a large bandwidth with sufficient spatial, spectral and temporal resolution is desired to allow true dynamic imaging spectroscopy. Such a practice and first results will be presented from Chapter 2 through Chapter 4.

\subsubsection{X-ray Emission}

\section{Bremsstrahlung radiation}

An introduction of thermal bremsstrahlung emission at radio wavelengths has been discussed in the previous section. As mentioned before, the radio emission is produced by electron-ion interactions with small deflections. To emit X-ray photons, strong interactions between electrons and ions in the solar atmosphere become the dominant process. There are, of course, bremsstrahlung between other particle species such as that between electrons and electrons, which will be discussed in Chapter 5. However, in the non-relativistic regime $\left(E_{e} \lesssim 300 \mathrm{keV}\right)$, the X-ray emission from solar flares is usually dominated by electron-ion interactions (Haug, 1975b; Kontar et al., 2007). Here I constrain myself to the electron-ion bremsstrahlung radiation only, following the discussions in Tandberg-Hanssen \& Emslie (1988).

The radiation is often categorized into thermal bremsstrahlung and non-thermal bremsstrahlung based on the energy distribution of the incident electron population. A flaring loop filled with hot plasma of $\sim 10 \mathrm{MK}$, in which the electrons have a thermal Maxwellian-Boltzmann distribution with a mean energy $\bar{E}_{e} \approx \bar{E}_{i}$, emits strong SXR (and also EUV) radiation which attributes to thermal bremsstrahlung radiation. In 
contrast, for emissions in the HXR range $(\sim 10-100 \mathrm{keV})$, non-thermal bremsstrahlung radiation is the dominant process under most circumstances, for which the source electrons are accelerated by the flare energy release to an average energy $\bar{E}_{e} \gg \bar{E}_{i}$ often having a power-law energy distribution.

For a uniform plasma with a temperature $T$ and an ion density $n_{i}$ distributed in a volume $V$, the thermal X-ray flux $F_{\text {thermal }}(\epsilon)$ received by an X-ray detector (photons $\mathrm{cm}^{-2} \mathrm{~s}^{-1} \mathrm{keV}^{-1}$ ) at a distance $R$ from the source can be obtained by integrating over all particles times the differential bremsstrahlung cross-section $\sigma(\epsilon, E)$

$$
F_{\text {thermal }}(\epsilon)=\frac{n_{i} V}{4 \pi R^{2}} \int_{\epsilon}^{\infty} f_{e}(E) v_{e}(E) \sigma(\epsilon, E) d E
$$

where $f_{e}(E)$ (electrons $\mathrm{cm}^{-3} \mathrm{keV}^{-1}$ ) is the differential electron density distribution at electron energy of $E, \sigma(\epsilon, E)$ is the physical parameter describing the effective area that governs the probability of bremsstrahlung radiation, which is discussed in detail in Koch \& Motz (1959). A widely used approximate expression is the non-relativistic solid-angle-averaged Bethe-Heitler (NRBH) cross-section

$$
\sigma_{\mathrm{NRBH}}(\epsilon, E)=\frac{\sigma_{0} \overline{Z^{2}}}{\epsilon E} \ln \frac{1+(1-\epsilon / E)^{1 / 2}}{1-(1-\epsilon / E)^{1 / 2}} \quad \mathrm{~cm}^{2} \mathrm{keV}^{-1}
$$

where $\sigma_{0}=7.90 \times 10^{-25} \mathrm{~cm}^{2} \mathrm{keV}$ and $\overline{Z^{2}}$ is the mean square atomic number of the target plasma $(\approx 1.4$ in the solar corona). The bremsstrahlung cross-section is zero at $\epsilon>E$ since an electron cannot emit a photon more energetic than the electron itself.

For a thermal plasma with a temperature $T$, the electron energy distribution has a Maxwellian-Boltzmann form described by Equation 1.6. The resulting X-ray flux $F_{\text {thermal }}(\epsilon)$ is proportional to the total volume emission measure $\xi_{V}=n_{e}^{2} V$. Usually the plasma in the source region is not isothermal but has a distribution over temperature, 
so that a differential emission measure $d \xi_{V} / d T$ is particularly useful.

A solar flare accelerates a significant number of non-thermal electrons. These electrons can result in strong emission at HXR energies via non-thermal bremsstrahlung radiation by interacting with the ambient plasma. Due to the nature of the radiation, the HXR flux is most intense where the density of target ions encountered by the nonthermal electrons is highest. As introduced in Section 1.2.2, in a solar flare, a strong HXR flux is usually observed when the downward propagating electron beams reach the dense chromosphere and lose most of their energy through Coulomb collisions. This case is the so-called "thick-target" bremsstrahlung case. The thick-target X-ray flux is given by

$$
F_{\text {thick }}(\epsilon)=\frac{S}{4 \pi R^{2}} \frac{1}{\bar{Z} K} \int_{\epsilon}^{\infty} f_{e}\left(E_{0}\right) v_{e}\left(E_{0}\right)\left(\int_{\epsilon}^{E_{0}} E \sigma(\epsilon, E) d E\right) d E_{0}
$$

where $f_{e}\left(E_{0}\right)$ is the electron density distribution for electrons with an energy of $E_{0}$ at the time of the injection, $S$ is the beam cross-sectional area, $K$ is a parameter consisting of the Coulomb logarithm for electron-electron collisions which is approximately a constant for X-ray-emitting electrons (see Brown 1971 for more details).

On the other hand, if the energy loss is not significant within the X-ray source region, the bremsstrahlung emission is called thin-target. In the solar context, HXR sources located in the corona have been frequently observed (see Krucker et al. 2008b for a review), for which thin-target bremsstrahlung emission is thought to be the dominant process under most circumstances. The corresponding X-ray flux is

$$
F_{\text {thin }}(\epsilon)=\frac{n_{i} V}{4 \pi R^{2}} \int_{\epsilon}^{\infty} f_{e}\left(E_{0}\right) v_{e}\left(E_{0}\right) \sigma\left(\epsilon, E_{0}\right) d E_{0}
$$

Note that the cross-section in the integral of the thin-target regime is a function of 
the initial electron energy $E_{0}$ but not the instantenous electron energy $E$ considering the energy loss as in the thick-target case.

For a uniform source and a single power-law electron density distribution of the form $f_{e}(E) \propto E^{-\delta}$, the resulting X-ray spectrum is also well approximated by a power law $F_{\gamma}(\epsilon) \propto \epsilon^{-\alpha}$. The relationship between the X-ray photon spectral index $\alpha$ and the input electron spectral index $\delta$ has a simple form for the non-relativistic Bethe-Heitler approximation of the bremsstrahlung cross-section:

$$
\alpha=\left\{\begin{array}{cl}
\delta+0.5 & \text { (thin-target) } \\
\delta-1.5 & \text { (thick-target) }
\end{array}\right.
$$

Note that the discussion above does not consider anisotropy of the X-ray-emitting electron distribution. Such consideration will be addressed in Chapter 5 .

\section{Inverse Compton Scattering}

Inverse Compton scattering (ICS) involves the scattering of low energy photons to higher energies by relativistic electrons. The net effect is that the photons gain and the electrons lose energy. ICS is one of the most common radiative processes in astrophysics, and is of course, ubiquitous in the solar context. A solar flare produces copius relativistic electrons. These electrons are able to upscatter optical, EUV, and SXR photons into HXR energies. Therefore, the ICS radiation should in principle contribute to the observed HXR photon flux.

A detailed discussion about ICS radiation and its applications in solar X-ray emission will be presented in Chapter 5. Here I just outline some of its salient features. Considering an electron of energy $\gamma m_{e} c^{2}$, where $\gamma=\sqrt{1-v^{2} / c^{2}}$ is the relativistic Lorentz factor, encountering a photon of energy $\epsilon_{1}$, the maximum energy 
of the upscattered photon is $4 \gamma^{2} \epsilon_{1}$. To within an order of unity the net effect of ICS is to transfer a low-energy photon to a high-energy photon with an energy gain of a factor $\gamma^{2}$. For example, to upscatter an optical photon of typical energy of $2 \mathrm{eV}$ into the HXR energy range (say, $20 \mathrm{keV}$ ), electrons with energies of $\sim 50 \mathrm{MeV}$ are required. Though it is difficult to accelerate electrons to such a high energy and such electrons are perhaps rare in number, there is good evidence that electrons can attain such energies in flares (e.g., Vilmer et al., 2003). Alternatively, upscattering a SXR photon of $1 \mathrm{keV}$ into a $20 \mathrm{keV}$ HXR photon requires only $\sim 2 \mathrm{MeV}$ electrons. Such electrons are many orders of magnitude more numerous than the $50 \mathrm{MeV}$ electrons, yet the photon density is also orders of magnitude lower than the optical photons. However, during a flare, the level of the incident EUV/SXR photon field is strongly enhanced, which is in favor of the ICS radiation.

Korchak $(1967,1971)$ considered the contributions from three possible mechanisms via which energetic electrons in solar flares can produce the observed X-ray emission: synchrotron, bremsstrahlung, and ICS. He concluded that the X-ray fluxes from electron-ion bremsstrahlung emission would dominate those from the other two mechanisms in most cases. In particular, synchrotron radiation is not favored as an emission mechanism for flare produced X-rays because the required electron energies should exceed $10 \mathrm{~s}$ of $\mathrm{GeV}$. The ICS, however, can play a role under some circumstances, especially when the ambient density in the source is low. The majority of X-ray studies in the past several decades has relied on the bremsstrahlung intepretation to, for example, deduce the properties of flare accelerated energetic electrons. In light of many recent observations that sometimes require extreme conditions in terms of bremsstrahlung radiation, reconsideration of the role of ICS radiation in flare X-ray $/ \gamma$-ray emissions seems to be necessary. Such discussions will be presented 
in Chapter 5.

\subsection{Instrumentation}

This dissertation utilizes observations at multiple wavelengths obtained from both ground- and space-based instruments, with a focus on radio and X-ray wavelengths. Multi-wavelength observations provide information on thermal and/or non-thermal particles originating from different layers of the solar atmosphere, which are particularly useful in disentangling the complexity of solar flare processes and the environment in which they occur. The primary data explored in this dissertation is from the Karl G. Jansky Very Large Array (VLA; Perley et al., 2011), the Frequency-Agile Solar Radiotelescope (FASR) Subsystem Testbed (FST; Liu et al., 2007b), the Reuven Ramaty High Energy Solar Spectroscopic Imager (RHESSI; Lin et al., 2002), and the Solar Dynamics Observatory (SDO; Pesnell et al., 2012).

- $V L A$ : The $V L A$ is a general-purpose radio interferometer located in west-central New Mexico, USA, operated by the National Radio Astronomy Observatory. It consists of 27 antennas of 25-m diameter, distributed along three arms. The array is reconfigurable - there are four standard configurations with maximum baseline lengths of $1,3.4,11$, and $36 \mathrm{~km}$, providing a variety of antenna distributions and a wide range of image resolution. It has been recently upgraded and now serves as the most powerful instrument currently available for solar radio observation. It can observe the Sun with unprecedented high spatial, spectral and temporal resolution with instantaneous bandwidth ratios as large as 2:1 in three different frequency bands: L (1-2 GHz), S (2-4 GHz), and C (4-8 GHz). Chapter 3 will discuss the use of the $V L A$ for solar observations in more detail. 
- FST: The FST is a three-element interferometer used as a prototype system for the next generation solar radioheliograph - the FASR. It uses the existing antenna system of the Owens Valley Solar Array(OVSA; Gary \& Hurford, 1994), which is a solar-dedicated interferometric array that is composed of two 27-m antennas and five 1.8-m antennas. The FST employs three of the five $1.8-\mathrm{m}$ antennas of the OVSA, but uses a new-generation backend system that allows Nyquist-limited high time and frequency resolution. Chapter 2.2 will discuss the detailed system configuration of the FST and the corresponding calibration strategies.

- RHESSI: The RHESSI is a NASA Small Explorer mission launched in February 2002. The space telescope is designed to perform imaging and spectral observations in X-ray and $\gamma$-ray wavelengths from $3 \mathrm{keV}$ to $17 \mathrm{MeV}$. It has a superb spatial resolution (up to $2^{\prime \prime} .3$ ) and spectral resolution (up to $1 \mathrm{keV}$ ). RHESSI imaging is achieved by Fourier transforming the time-modulating X-ray flux created by nine rotating subcollimators with different slit-widths (Hurford et al., 2002). Images can be made using different reconstruction methods, including CLEAN, Maximum Entropy Methods (MEM), Forward Fitting, and Pixon, for user-selected energy and time ranges with a combination of subcollimators to optimize image resolution. Spectroscopy is carried out using the Solarsoft package OSPEX (Schwartz et al., 2002), which corrects for the instrumental response in converting the recorded photon-count spectrum to the emitted photon spectrum from the Sun. A background subtraction is also required to remove the solar background and instrumental noise.

- $S D O$ : The $S D O$ is a space-based solar telescope as part of the NASA Living With a Star program, and was launched in February 2010. The spacecraft 
includes three instruments: the Atmospheric Imaging Assembly (AIA; Lemen et al., 2012), the Helioseismic and Magnetic Imager (HMI; Scherrer et al., 2012), and the Extreme Ultraviolet Variability Experiment (EVE; Woods et al., 2012). The AIA provides full-disk imaging of the Sun in ten white light, ultraviolet (UV) and EUV bands at high spatial (1".5) and temporal resolution (12 s), which samples plasma of the solar atmosphere at various characteristic temperatures from $5000 \mathrm{~K}$ to $2 \times 10^{7} \mathrm{~K}$. The HMI takes high-resolution measurements of the longitudinal and vector magnetic field of the photosphere, which has significant inputs in characterizing the solar magnetic field in order to understand the Sun's interior as well as solar activities. The EVE measures the Sun's EUV irradiance with excellent spectral and time resolution, important in understanding the solar EUV variations and magnetic changes on the Sun.

- Hinode: The Hinode is a joint mission of Japan, Europe and USA (Kosugi et al., 2007). It consists of three different instruments: the X-Ray Telescope (XRT; Golub et al., 2007), a SXR imager sampling the solar corona's hottest component (0.5 to $10 \mathrm{MK})$, the Solar Optical Telescope (SOT; Tsuneta et al., 2008), providing high-resolution $\left(0^{\prime \prime} .2-0^{\prime \prime} .3\right)$ images in optical wavelengths as well as sensitive vector magnetogram of the photosphere, and the ExtremeUltraviolet Imaging Spectrometer (EIS; Kosugi et al., 2007), offering spatially resolved spectra in two EUV bands.

- Other instruments: The Solar TErrestial RElations Observatory (STEREO; Kaiser et al., 2008), a set of two satellites - one ahead of Earth in its orbit, the other trailing behind-provides observations of the Sun in optical, EUV, and radio wavelengths from two different perspectives. The GOES are a series of satellites operated by the U.S. National Oceanic and Atmospheric Adminis- 
tration (NOAA), supporting meteorological observing and space environment monitoring. The space environment monitoring system consists of an X-ray sensor, a particle sensor, and a magnetometer. The GOES X-ray data provide a full-Sun-integrated SXR flux for the $0.5-3 \AA(0.5-4 \AA$ prior to GOES-8) and 1-8 $\AA$ bands. GOES numbered 12 and above also carry a solar X-ray imager (SXI) used for full-Sun imaging.

\subsection{Scientific Goal and Dissertation Outline}

As mentioned in Section 1.1, solar flares are among the most powerful explosive events and particle accelerators known in the solar system, and have been identified as a strong source responsible for the space weather that affects our everyday life on the Earth. A detailed knowledge of the fundamental energy release processes in solar flares is critical in understanding their various influences and in making predictions of the space weather. In addition, fundamental physical processes in solar flares - magnetic energy storage, impulsive energy release and particle acceleration - are ubiquitous throughout the Universe, such as the magnetic diffusion region in Earth's magnetotail (Deng \& Matsumoto, 2001; Øieroset et al., 2001; Phan et al., 2006), flares, jets and CMEs on the Sun, flares on the other stars (Bastian \& Bookbinder, 1987; Bastian, 1994b; Maehara et al., 2012), superflares from magnetars (Hurley et al., 2005; Palmer et al., 2005; Terasawa et al., 2005), flashes from accretion disks of AGNs (Tavani et al., 2009), etc. The fundamental physics of magnetic reconnection is even important in controlled nuclear fusion. This is one mechanism preventing magnetic confinement of the fusion fuel. The Sun's proximity allows us to exploit it as an exceptional laboratory to study those physical processes in the greatest detail.

There has been significant progress in the area of solar flare studies for the past 
decades, thanks to the new observing facilities, theoretical advances, and improved numerical modeling techniques. Yet our understanding of the energy release processes in solar flares is still incomplete. Outstanding questions include:

- Where and how is the magnetic energy released?

- What are the characteristics of the energetic particles accelerated by the released energy and what mechanisms are responsible for their radiation?

- How is the released energy transported in the coupled layers of the solar atmosphere?

- What are the properties of the plasmas in and around the energy release site, including their density, temperature, magnetic field strength and configuration?

This dissertation seeks answers to these questions via studies of radio and X-ray emission from solar flares, aided by other multi-wavelength observations in optical, UV/EUV, and SXR wavelengths. In particular, as mentioned in Section 1.2.3, spatially resolved dynamic spectroscopy is exploited, for the first time, in studying solar coherent radio emission. Chapter 2 describes a study on a zebra-pattern burst using spatially resolved dynamic spectroscopy provided by a three-element array - the FST. Though imaging is impossible, spatial information of every pixel on the dynamic spectrum can be obtained, allowing us to obtain the source location, spatial extension, and its dynamical evolution. The relevant emission mechanisms of the burst are examined and the relation of the burst to the flare magnetic energy release is suggested. This work allows me to become familiar with radio interferometry and forms a basis for the subsequent studies employing true dynamic imaging spectroscopy. In Chapter 3, I describe commissioning of the VLA "solar mode", which enables the general-purpose radio telescope to perform solar observations. Based on test results 
of the $V L A$ 's hardware, solar observing and calibration strategies are suggested. The upgraded $V L A$ allows true dynamic imaging spectroscopic observations of the Sun, meaning a solar radio image can be produced for every pixel on the dynamic spectrum. Chapter 4 presents the first results using this technique. Dynamic imaging spectroscopic observations of dm- $\lambda$ type III radio bursts enable detailed trajectories of propagating electron beams in the low corona to be deduced. Together with magnetic, EUV, and HXR data, the observations allow us to derive the properties of the energy release and the surrounding medium. We show that the observations are qualitatively consistent with the standard scenario of energy release. We conclude that the magnetic energy release is highly fragmentary and that the surrounding coronal medium for conducting the type-III-burst-emitting electron beams is fibrous in nature. Chapter 5 considers X-ray emission mechanisms. We investigate whether or not ICS could be an alternative emission mechanism for coronal HXR sources other than the usually-assumed thin-target bremsstrahlung emission, which would open a new window in diagnosing the flare energy release. Finally, I summarize the major results and discuss future work in Chapter 6. 


\section{Chapter 2}

\section{Zebra-Pattern Radio Bursts:}

\section{Coherent Emission From Trapped}

\section{Electrons*}

*Adapted from Chen et al. (2011). I contributed to most of the data reduction, scientific analysis, and writing with the major help from Tim Bastian. Dale Gary provided the FST and OVSA data and helped with the calibration. Ju Jing performed the magnetic field extrapolation. 


\section{$2.1 \quad$ Introduction}

Fine structures in the solar radio bursts - in both the time and frequency domainshave been studied for many years. They are believed to embody important information about charged particle acceleration processes, particle dynamics, and emission mechanisms (Fleishman et al., 1994). Many such fine structures - type III bursts and their variants, spike bursts, pulsations, fiber bursts - are believed to be the result of non-equilibrium processes in the coronal plasma. Zebra-pattern radio bursts (Slottje, 1972, hereafter "zebra pattern" will be abbreviated to "ZP") are one of the most striking examples of such fine structures.

The observed properties of $\mathrm{ZP}$ radio bursts have been presented in detail in the review by Chernov (2006) and are reiterated briefly here. ZP bursts appear in radio dynamic spectra as closely spaced, quasi-parallel bands of emission, typically ranging from $\sim 5$ to 20 in number but sometimes showing as many as 70 . They have been observed at meter wavelengths for decades (Elgaröy, 1959; Slottje, 1972; Kuijpers, 1975); more recently, similar structures have been reported at decimeter and centimeter wavelengths. For the purposes of discussion we denote the instantaneous frequency of a single ZP emission band or stripe by $\nu_{e}$, the frequency bandwidth of a ZP emission band by $\Delta \nu_{e}$, the separation between adjacent ZP emission bands by $\Delta \nu_{s}$, the mean frequency of two adjacent emission bands as $\nu_{m}$, the mean frequency of the ZP emission bands as a whole by $\left\langle\nu_{e}\right\rangle$, and the overall frequency bandwidth occupied by ZP emission bands by $\Delta \nu_{\text {tot }}$. Generally, $\Delta \nu_{\text {tot }} /\left\langle\nu_{e}\right\rangle$ decreases with frequency whereas $\Delta \nu_{s} /\left\langle\nu_{e}\right\rangle$ increases with frequency; the relative bandwidth of individual ZP emission bands $\Delta \nu_{e} / \nu_{e}$ shows no obvious trend with frequency and is typically $\lesssim 1 \%$ (Chernov, 2006). There are few reports of the brightness temperature $T_{B}$ of $\mathrm{ZP}$ bursts. In those cases where such constraints are available, the brightness tempera- 
ture is typically very high. Chernov et al. (1994) estimated the $T_{B}$ of a metric ZP to be $\approx 10^{10} \mathrm{~K}$ with the source size constrained by the Nançay Radioheliograph (NRH), In Chernov et al. (2003), a decimetric ZP that consisted of spiky superfine structures was estimated to have $T_{B} \gtrsim 10^{13} \mathrm{~K}$ by assuming the burst had the same source size as a spike burst. Altyntsev et al. (2005) used the Siberian Solar Radio Telescope (SSRT) to observe a ZP burst at $\approx 5.7 \mathrm{GHz}$, the highest frequency ever reported for ZP emission, which yielded a lower limit of $T_{B} \approx 2 \times 10^{7} \mathrm{~K}$, the source size being $\lesssim 10^{\prime \prime}$. ZP bursts are typically observed during the impulsive and/or decay phases of the flares. They are typically polarized in the sense of the ordinary wave mode and the degree of polarization can be very high. The durations of ZP bursts can vary from a few minutes down to a few seconds at meter wavelengths to decimeter/centimeter wavelengths, respectively. The narrow band features, high degree of circular polarization, and indications of high brightness temperatures suggest that the corresponding emission mechanism is coherent.

ZP bursts often appear with the presence of type IV continuum emission (hereafter "continuum"). Many other fine structures, including type III bursts, broadband pulsations, fiber bursts, and spikes accompany, or are associated with, ZP emission. There are also some rare examples where ZPs consist of pulsating superfine structures (Kuznetsov \& Tsap, 2007; Chen \& Yan, 2007; Kuznetsov, 2008), and they appear in fast-drift, type-III-like, absorption features (Zlotnik et al., 2009), which could be related to fast electron beam injections into the magnetic trap.

There is no broadly accepted interpretation for ZP emission. Several types of models purport to explain the ZP phenomenon (see Chernov, 2006; Zlotnik, 2009). Most involve the growth and conversion of electrostatic wave modes to transverse modes. One type of model suggests that all the ZP stripes originate from the same 
discrete source, the dimensions of which are assumed to be small enough for the inhomogeneity of the plasma density and the magnetic field to be neglected. In these models, zebra stripes are assumed to be simultaneously generated at several harmonics of the local electron cyclotron frequency, due to nonlinear couplings of Bernstein waves with each other or with upper-hybrid waves (hereafter "Bernstein models"; e.g. Rosenberg, 1972; Zheleznyakov \& Zlotnik, 1975a,b; Zaitsev \& Stepanov, 1983).

A second type of ZP model is based on trapping upper-hybrid $z$-mode waves in density inhomogeneities (LaBelle et al., 2003). The trap results in a discrete spectrum of eigenfrequencies. The model depends on the emission by many such discrete traps distributed over a larger volume.

Models based on propagation phenomena have also been proposed. Bárta \& Karlický (2006) and Laptukhov \& Chernov (2006) suggest that coronal fine structure can behave as an optical filter or produce Bragg-like reflections, resulting in regular emission bands. Alternatively, interference between direct and reflected rays from a coherent source have been suggested (Ledenev et al., 2006; Tan, 2010).

Another class of models argues that ZPs are related to an extended source filled with energetic electrons. The different zebra stripes originate from different locations in the extended source, where resonance conditions are fulfilled. The most popular model of this kind is the so-called double plasma resonance (DPR) model, first proposed by Zheleznyakov \& Zlotnik (1975a,b), and subsequently developed by several authors (e.g., Winglee \& Dulk, 1986; Kuznetsov \& Tsap, 2007). In this class of models, upper-hybrid waves are generated most efficiently at locations where the DPR occurs:

$$
\nu_{\mathrm{uh}}=\left(\nu_{\mathrm{pe}}^{2}+\nu_{\mathrm{ce}}^{2}\right)^{1 / 2}=s \nu_{\mathrm{ce}}
$$


where $\nu_{\mathrm{uh}}, \nu_{\mathrm{pe}}$, and $\nu_{\mathrm{ce}}$ have been defined in Chapter 1.2.3 as the upper-hybrid frequency, electron plasma frequency, and electron cyclotron frequency, respectively, and $s$ is the harmonic number. The distribution of the DPR levels in the flare loop is determined by spatial gradients in the plasma density and magnetic field.

Finally, models are based on propagation of whistler wave packets (Chernov, 1976, 1990) across, or along, the magnetic trap where the energetic electrons generate Langmuir waves (hereafter "whistler model"). ZPs are produced by coalescence of the Langmuir waves $(l)$ and whistlers $(w)$ through the process $l+w \longrightarrow t$, where $t$ stands for transverse waves that can be observed as emission near the local plasma frequency. They propose that ZPs as a whole are the manifestation of the ensemble of periodically generated whistler wave packets propagating in the magnetic trap, in which each zebra stripe corresponds to one propagating whistler wave packet. In this way, zebra stripes can be separated regularly from each other in height (and emit at different frequencies) by a distance determined by the whistler propagation velocity and time interval of generating the whistlers.

Both Chernov (2006) and Zlotnik (2009) discuss each of these models and summarize their strengths and weaknesses. Many display significant theoretical shortcomings. In light of these shortcomings, Chernov favors whistler models. In contrast, Zlotnik favors DPR models. We will therefore direct most of our attention toward the last two classes of models-DPR and whistler models - in subsequent discussion.

As for the type IV continuum emission associated with ZP bursts, it is assumed by most models that it arises from fast electrons trapped magnetically in the coronal loops. However, the relationship between the continuum and ZP emission varies from model to model. In the Bernstein and DPR models, it is suggested that the only difference between their formation is whether these trapped electrons favor conditions 
for creating the zebra pattern or not, which is most likely in the form of anisotropic momentum distributions. In the whistler model, the $\mathrm{ZP}$ is formed through interactions of the plasma waves that can result in the type IV continuum. The propagation models, however, suggest that either the ZP sources reside in the continuum source (Ledenev et al., 2006) or ZP emission as well as the "background" continuum as a whole are just type IV continuum emission modulated by the inhomogeneous medium (Bárta \& Karlický, 2006; Laptukhov \& Chernov, 2006). However, because of the lack of spatial information, the physical relationship between the emission sources of ZPs and type IV continuum is still not known.

The means of placing meaningful constraints on models for ZP and continuum emission has been limited by the unavailability of spatial resolution at each of the frequencies and times recorded by the dynamic spectrum. Interferometric observations of ZP emission at even a single frequency are relatively few in number. Several examples are provided by fixed-frequency observations of ZP by the $N R H$ in combination with observations made by a spectrometer (e.g., Chernov et al., 1994, 1998, 2001; Aurass et al., 2003; Chernov, 2005). Other examples have been provided by Altyntsev et al. (2005) and Chernov et al. (2005, 2006) who combined interferometric observations of ZP made by the $S S R T$ at $5.7 \mathrm{GHz}$ with spectroscopic observations obtained by the Chinese Solar Broadband Radio Spectrometer (SBRS).

In the present paper we describe the first interferometric observations of ZP emission for which both high-time resolution and high-spectral resolution observations are simultaneously available over a significant frequency bandwidth. The relevant instrumentation is described in Section 2.2. The ZP event and its analysis are described in Section 2.3. The event is placed in a physical context in Section 2.4, where we argue that the data are consistent with a DPR model. We conclude in Section 2.5. 


\subsection{Instrumentation}

The observations were obtained by the Frequency-Agile Solar Radiotelescope (FASR) Subsystem Testbed (FST) (Liu et al., 2007b). FASR is a next generation solar radio telescope (Bastian, 2004) designed to provide simultaneous imaging and spectroscopic observations over a large bandwidth, with high angular, time, and spectral resolutions. The FST is a prototype and testbed system for FASR. As such, it is the first system with the ability to combine Nyquist-limited high-time and frequency resolution with interferometric ability to locate sources (Liu et al., 2007b).

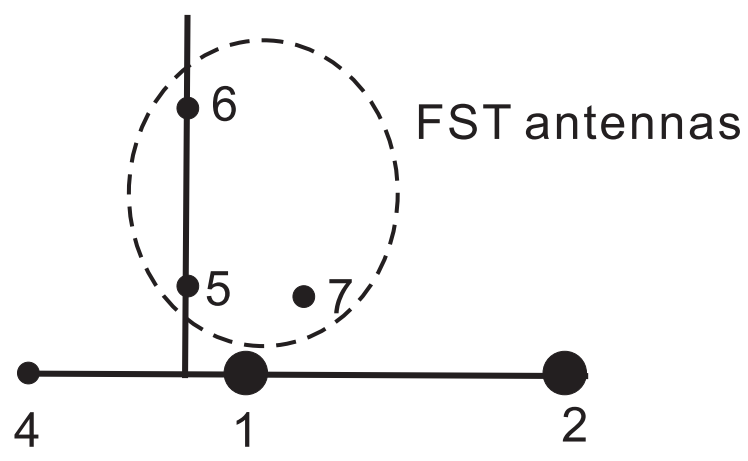

Fig. 2.1. - Nearly right triangle antenna configuration of FASR Subsystem Testbed, which consists of three $1.8 \mathrm{~m}$ antennas numbered 5, 6, and 7 of the Owens Valley Solar Array (adapted from Liu, 2007).

The FST uses the existing antenna system of the Owens Valley Solar Array (OVSA; Gary \& Hurford, 1994). OVSA is a solar-dedicated interferometric array that is composed of two $27 \mathrm{~m}$ antennas and five $1.8 \mathrm{~m}$ antennas. OVSA can observe the Sun at up to 86 frequencies in the range 1-18 GHz. The FST employs three 
of the $1.8 \mathrm{~m}$ OVSA antennas (antenna numbers 5, 6, and 7) as shown in Figure 2.1. The longest baseline, between antennas 6 and 7 , is nearly $280 \mathrm{~m}$ which yields a minimum fringe spacing of $221^{\prime \prime}$ at $1 \mathrm{GHz}$. Liu et al. (2007b) describe the FST system configuration in detail, and we briefly reiterate it here. A radio frequency $(\mathrm{RF})$ splitter divides the output signals of the three OVSA/FST antennas to simultaneously feed the OVSA receivers and the FST. Thus, the FST can be used in parallel with $O V S A$, observing the same source as $O V S A$, without affecting $O V S A$ 's normal operation. FST signals are amplified and transmitted by a broadband optical fiber to a block down-converter. The 1-5 GHz or 5-9 GHz band of RF signal from each antenna is selected and down-converted to 1-5 GHz as necessary. The spectral-line down-converter is used to select a single-sideband, $500 \mathrm{MHz}$ band which is tunable anywhere within the $1-5 \mathrm{GHz}$ band. The selected $500 \mathrm{MHz}$ band is further downconverted to an intermediate frequency (IF) band of 500-1000 MHz. The $500 \mathrm{MHz}$ IF band is sampled at 1 Gsps and the data are written to disk. The full-resolution time-domain data are then correlated offline using a software correlator (written in IDL) to produce amplitude and phase spectra on the three interferometric baselines. For daily solar observing, the system employs a time resolution of $\approx 20 \mathrm{~ms}$ and a frequency resolution of $0.98 \mathrm{MHz}$.

The small size of the FST antennas precludes calibration of the FST against sidereal radio sources. However, since the event was observed by both FST and OVSA in parallel, we can make use of the OVSA observations to cross-calibrate FST. OVSA is calibrated against sidereal standards in order to determine the complex gain of each antenna. However, OVSA only samples the RF spectrum at 1.2 and $1.4 \mathrm{GHz}$. Moreover, the $1.2 \mathrm{GHz} O V S A$ data were found to be corrupted and were therefore unusable. Therefore, the FST $1.4 \mathrm{GHz}$ data were averaged in frequency and time 
to match the OVSA data. The calibrated OVSA amplitudes and phases of antenna baselines 5-6, 6-7, and 5-7 were then compared to those measured by FST, and the FST amplitudes and phases were corrected accordingly. As Figure 2.2 shows, they agree with each other quite well after the cross-calibration. However, this process does not directly allow bandpass calibration of FST. Based on an examination of broadband continuum emissions that occupy the entire frequency band, we conclude that the FST has approximately linear bandpass patterns in phase with good stability in time on all three baselines. We therefore applied linear fits as the bandpass correction to the phases.
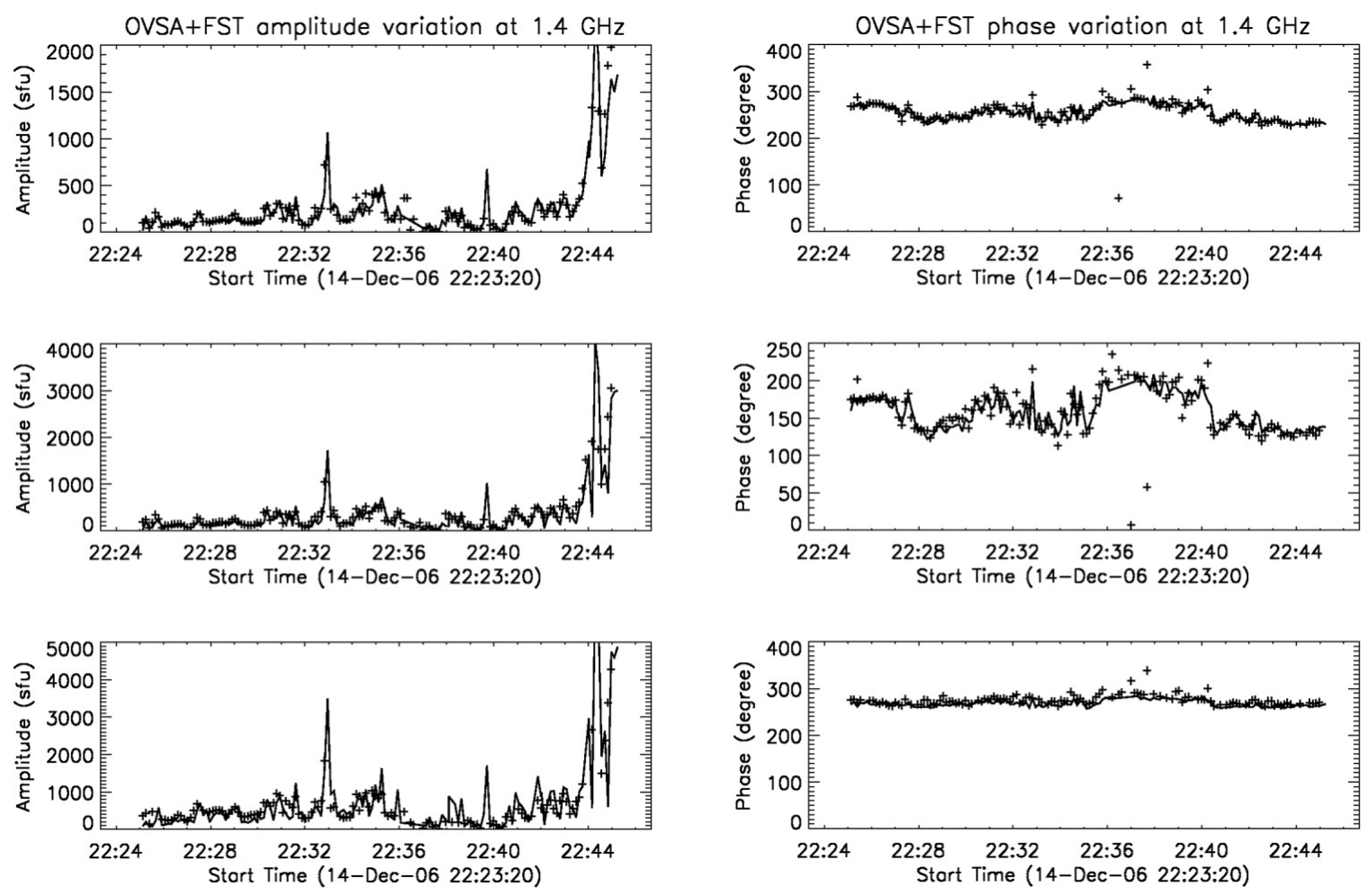

Fig. 2.2. - Comparison of OVSA and FST amplitudes and phases (OVSA: plus; FST: solid line). From top to bottom: baselines of antenna 5-6, 6-7, 7-5, respectively. 


\subsection{Observations}

The FST observed the powerful Geostationary Operational Environmental Satellite (GOES) class X1.5 flare that occurred on 2006 December 14 in NOAA/USAF active region 10930 at S06W46, the site of an X3.4 flare the previous day (see, e.g., Su et al., 2007). The flare on December 14 was accompanied by a fast halo coronal mass ejection (CME) and a solar energetic particle event. Figure 2.3(a) shows the GOES SXR light curve; the flare started at 21:07 UT, peaked at 22:15 UT, and ended at around 04:00 UT on December 15. The radio time profiles at 1.415 GHz, 2.695 GHz, and $8.8 \mathrm{GHz}$ obtained by the Radio Solar Telescope Network (RSTN, operated by the U.S. Air Force) are shown in Figure 2.3(b)-(d). Note the difference in scale between the intense $1.415 \mathrm{GHz}$ emission and that at 2.695 and $8.8 \mathrm{GHz}$.

The two X-class flares on 2006 December 13 and 2006 December 14 were observed by the X-ray Telescope (XRT; Golub et al., 2007) and the Solar Optical Telescope (SOT; Tsuneta et al., 2008) aboard Hinode (Kosugi et al., 2007). Most of the XRT images were taken with a $523^{\prime \prime} \times 523^{\prime \prime}$ field of view $(\mathrm{FOV})$ at a resolution of $1^{\prime \prime} .021$ pixel $^{-1}$. Vector photospheric magnetograms were obtained by the SOT SpectroPolarimeter (SP). The slit length and width of SOT/SP are $164^{\prime \prime}$ and $0^{\prime \prime} .16$, respectively. For each magnetogram with a $297^{\prime \prime} \times 164^{\prime \prime} \mathrm{FOV}$, the slit scanned from east to west for about $1 \mathrm{hr}$, with a resolution of $0^{\prime \prime} .297$ and $0^{\prime \prime} .320$ in the east-west and north-south directions, respectively. The SOT Ca II H band samples the chromospheric structure at a very high spatial resolution $\left(0^{\prime \prime} .109\right.$ pixel $\left.^{-1}\right)$. Like $\mathrm{H} \alpha$ this band is sensitive to plasma heating by precipitating electrons and/or thermal conduction. The images were taken with a $223^{\prime \prime} \times 111^{\prime \prime}$ FOV with a cadence of 120 s. Figure $2.4(\mathrm{a})$ and (b) give the longitudinal magnetogram at the photospheric level $B_{z}(0)$ observed from 22:00:05 to 23:03:16 UT and an example of a Ca II H image at 22:37:35 UT. 

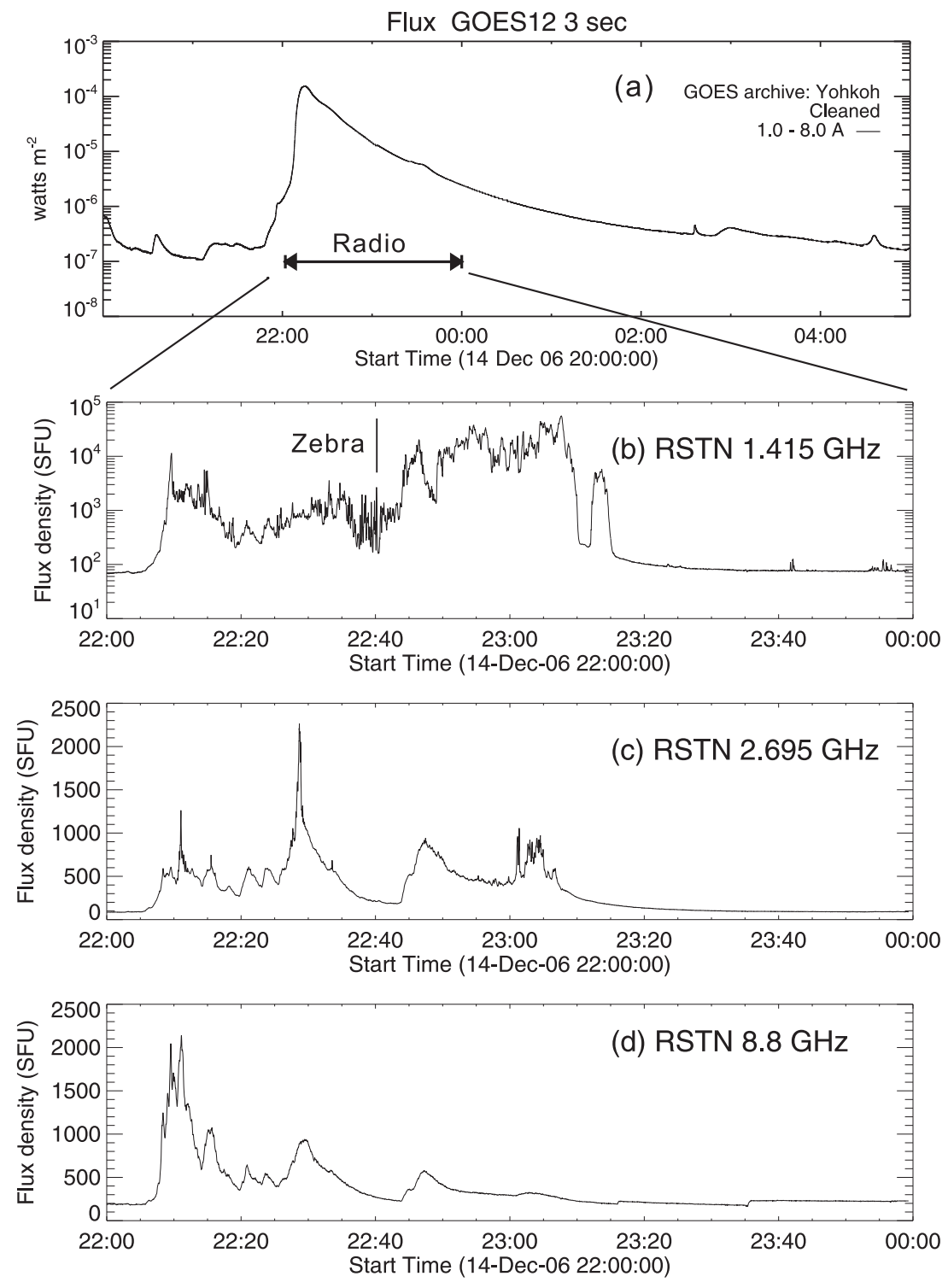

Fig. 2.3. - GOES and RSTN time profiles of the 2006 December 14 flare. Note the difference in scale between the intense $1.415 \mathrm{GHz}$ emission and that at 2.695 and 8.8 GHz. The time of the ZP event is marked by the vertical line in (b), at about 22:40 $\mathrm{UT}$, during the decay phase. 

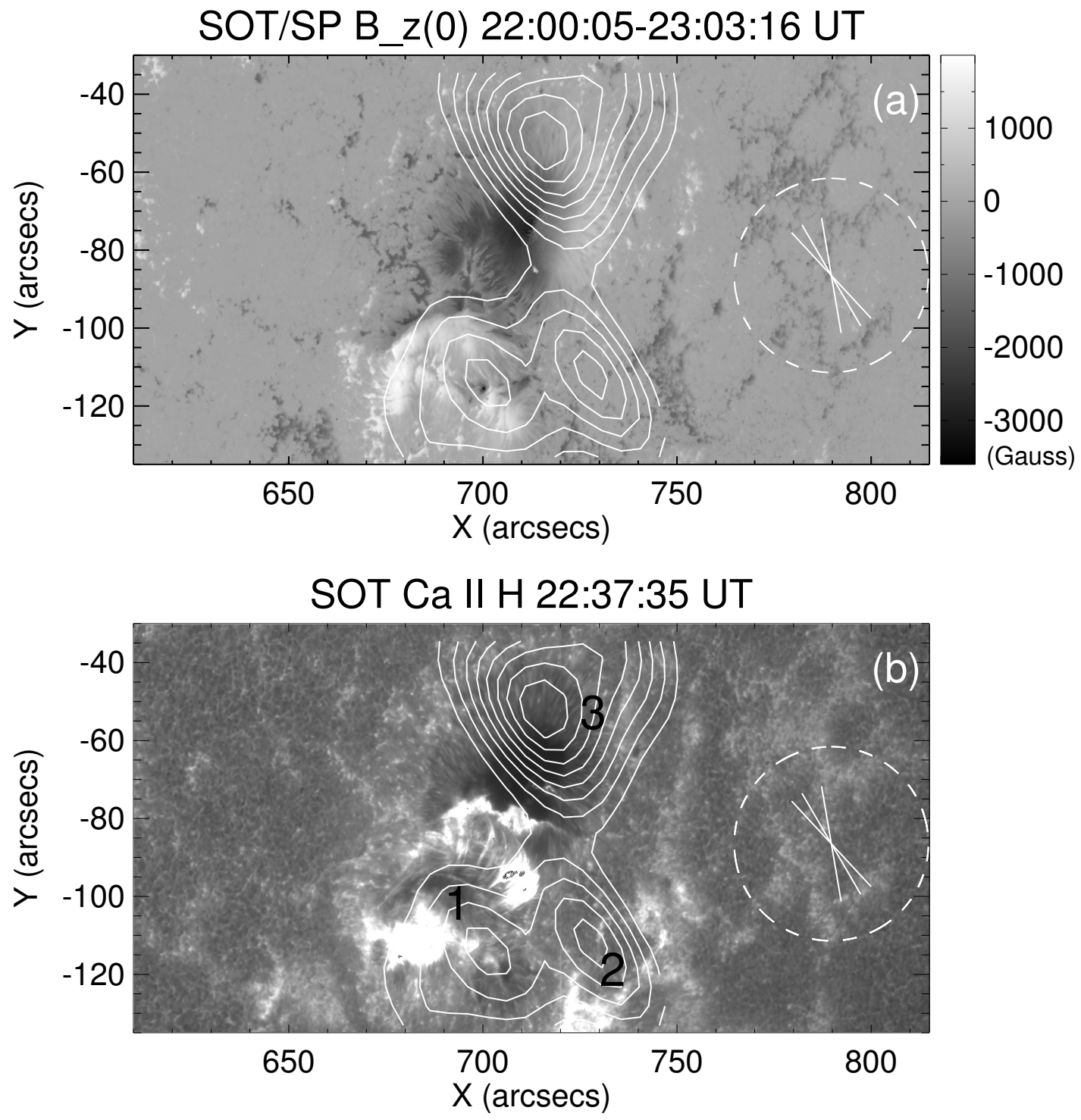

Fig. 2.4.- Longitudinal photometric magnetogram $B_{z}(0)$ (a) and an example of a Ca II H image at 22:37:35 UT (b) by SOT. North is up and west is to the right. The magnetogram was observed from 22:00:05 to 23:03:16 UT for about $1 \mathrm{hr}$. The intersection of the three interferometric fringes of FST denotes the location of the ZP emission centroid (observed around 22:40 UT), with a dashed circle representing the $\approx 50^{\prime \prime}$ apparent source size obtained in Section 2.3.1. The error of the source location is between $1^{\prime \prime} .3$ and $3^{\prime \prime} .5$ depending on direction. The contours are the OVSA 4.6-6.2 $\mathrm{GHz}$ map at the same time of the ZP (the levels have an increment of $10 \%$ of the maximum). The numbers "1", "2", and "3" in (b) mark the locations of the three major Ca II H bright regions seen in Figure 2.5(a)-(c). 
Figure 2.5 shows the Ca II H (Figures 2.5(a)-(c)) and XRT (Figures 2.5(d)-(f)) images at times prior to the flare (a and d), during the flare maximum (b and e), and during the decay phase ( $\mathrm{c}$ and $\mathrm{f}$ ) at the time of the ZP. The core flare region (marked as region "1" in Figure 2.4(b)) is located between the two sunspots with opposite polarity. According to Su et al. (2007), the flare loops inside this region evolve from highly sheared in the pre-flare phase into less sheared in the post-flare phase, based on the XRT observations. Except for the flare core region, there are two other major bright regions in the Ca II H images, where the brightness of SXR loops is enhanced at the same time, as shown in the XRT images. One is located about $50^{\prime \prime}$ to the west of the core region (region "2" in Figure 2.4(b)), and another is located to the northwest (NW) near the larger sunspot with negative polarity (region "3" in Figure $2.4(\mathrm{~b}))$.

A more subtle Ca II H brightening is observed at the time of the ZP (at about 22:40 UT), indicated by a white arrow in Figure 2.5(c). This brightening appeared in Ca II H images at around 22:10 UT (near the flare maximum) and persisted for more than $1 \mathrm{hr}$.

\subsubsection{FST ZP Observations}

The FST observed the 2006 December 14 flare over a frequency range of 1.0-1.5 GHz. The instrument observed both right-circularly polarized (RCP) and left-circularly polarized (LCP) radiation, switching between the two polarizations every $4 \mathrm{~s}$. A spectrum of 511 channels across the $500 \mathrm{MHz}$ bandwidth was produced every $\approx 20$ ms. The 2006 December 14 flare produced a high level of type IV burst activity in the post-flare phase for more than $1 \mathrm{hr}$. A rich variety of fine structures was observed in the 1.0-1.5 GHz band, including ZP, fiber bursts, pulsations, and others. The time 

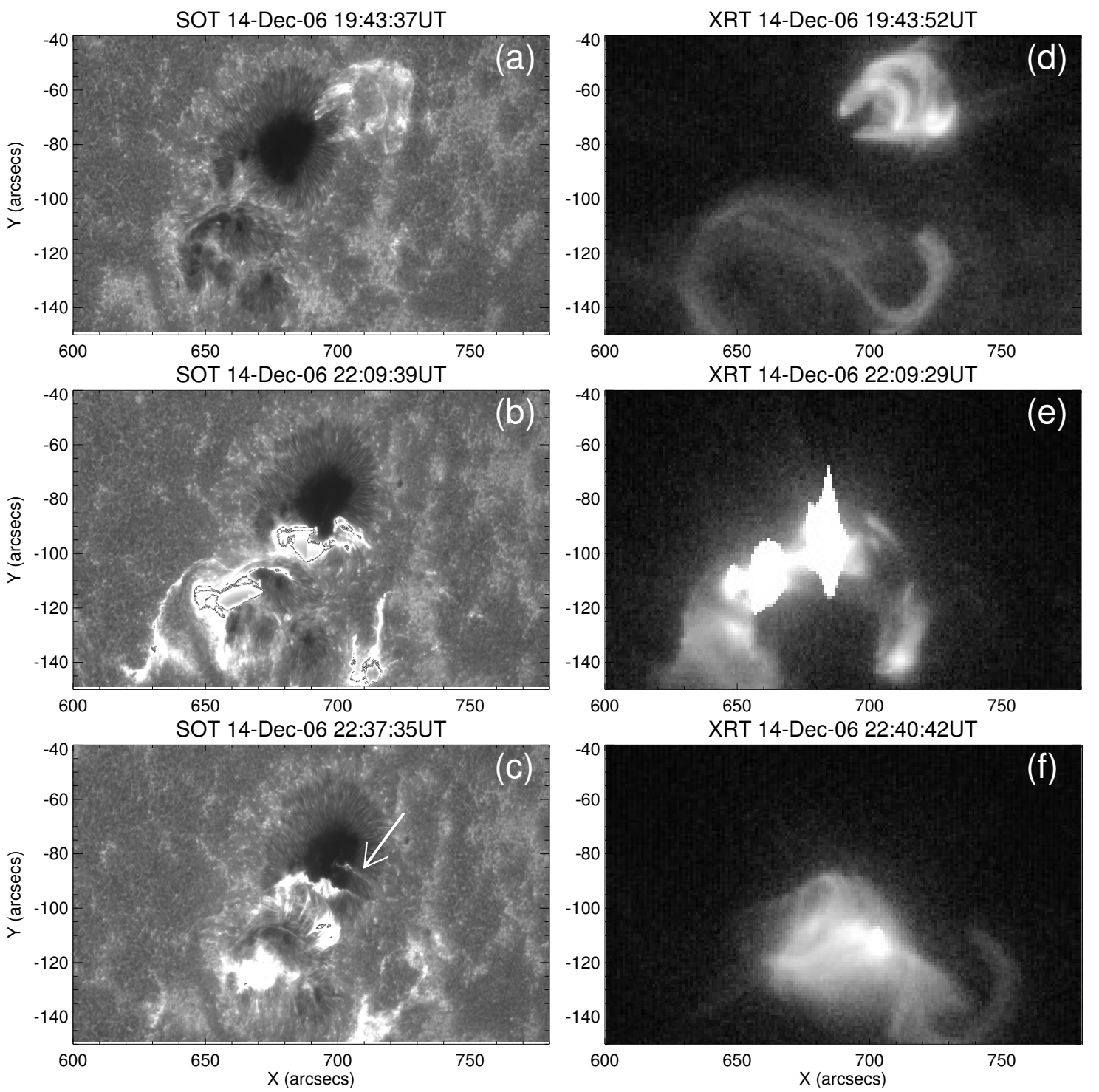

Fig. 2.5. - Hinode SOT Ca II H and XRT observations of the 2004 December 14 flare showing the chromospheric and coronal evolution. Left column: Hinode Ca II H images at 19:43:37, 22:09:39, and 22:37:35 UT. Right column: Hinode XRT images at 19:43:52, 22:09:29, and 22:40:42 UT. They correspond to the times prior to the flare, during the flare maximum, and of the occurrence of $\mathrm{ZP}$ in the post-flare phase. The white arrow in (c) shows the subtle Ca II H brightening that may be magnetically associated with the ZP source. 
of the ZP event is marked by the vertical line in Figure 2.3(b), at about 22:40 UT, during the decay phase.

\section{Total Power Dynamic Spectrum}

Figure 2.6(a) shows a dynamic spectrum of roughly $1 \mathrm{~s}$ of total power data near 22:40:07 UT. A striking ZP radio burst is present. The data are RCP; the LCP observations made just prior to those presented here showed no ZP emission. We conclude that the ZP burst is highly RCP. A precise measure of the degree of polarization is not possible, however, because the noise in the LCP observations is dominated by polarization leakage from the RCP channel. The ZP burst shows as many as 12 distinct bands or stripes superposed on a type IV-like continuum background centered on $\left\langle\nu_{e}\right\rangle \approx 1.32 \mathrm{GHz}$ and with a frequency bandwidth $\Delta \nu_{\text {tot }}$ of up to $150 \mathrm{MHz}$, or $\Delta \nu_{\text {tot }} /\left\langle\nu_{e}\right\rangle \gtrsim 10 \%$. All of the zebra stripes drift in frequency together irregularly with time. Figure 2.6(c) shows a histogram of the drift rates of the zebra stripes in the dashed box marked in Figure 2.6(a). An average drift rate of about $-50 \mathrm{MHz} \mathrm{s}{ }^{-1}$ is indicated by the vertical line. The overall trend is to drift from higher to lower frequencies. The drift rates of the zebra stripes are mainly between $-100 \mathrm{MHz} \mathrm{s}^{-1}$ to $100 \mathrm{MHz} \mathrm{s}^{-1}$, comparable to, but generally slower than, those of the so-called fiber burst in the same frequency range (Elgarøy, 1982).

The frequency profile of the ZP emission (averaged in the time denoted by the small solid box in Figure 2.6(a)) is shown in Figure 2.6(b). The contrast of each stripe is defined by $\left(I_{\mathrm{on}}-I_{\mathrm{off}}\right) / I_{\mathrm{off}}$ (where $I_{\mathrm{on}}$ and $I_{\mathrm{off}}$ are the intensities "on" and "off" a zebra stripe, respectively) and is shown in Figure 2.6(d). The relative frequency separations between adjacent zebra stripes $\Delta \nu_{s} / \nu_{m}$ in the dashed box of Figure 2.6(a) are shown in Figure 2.13(a) as diamonds. They are color-coded in time from blue to 

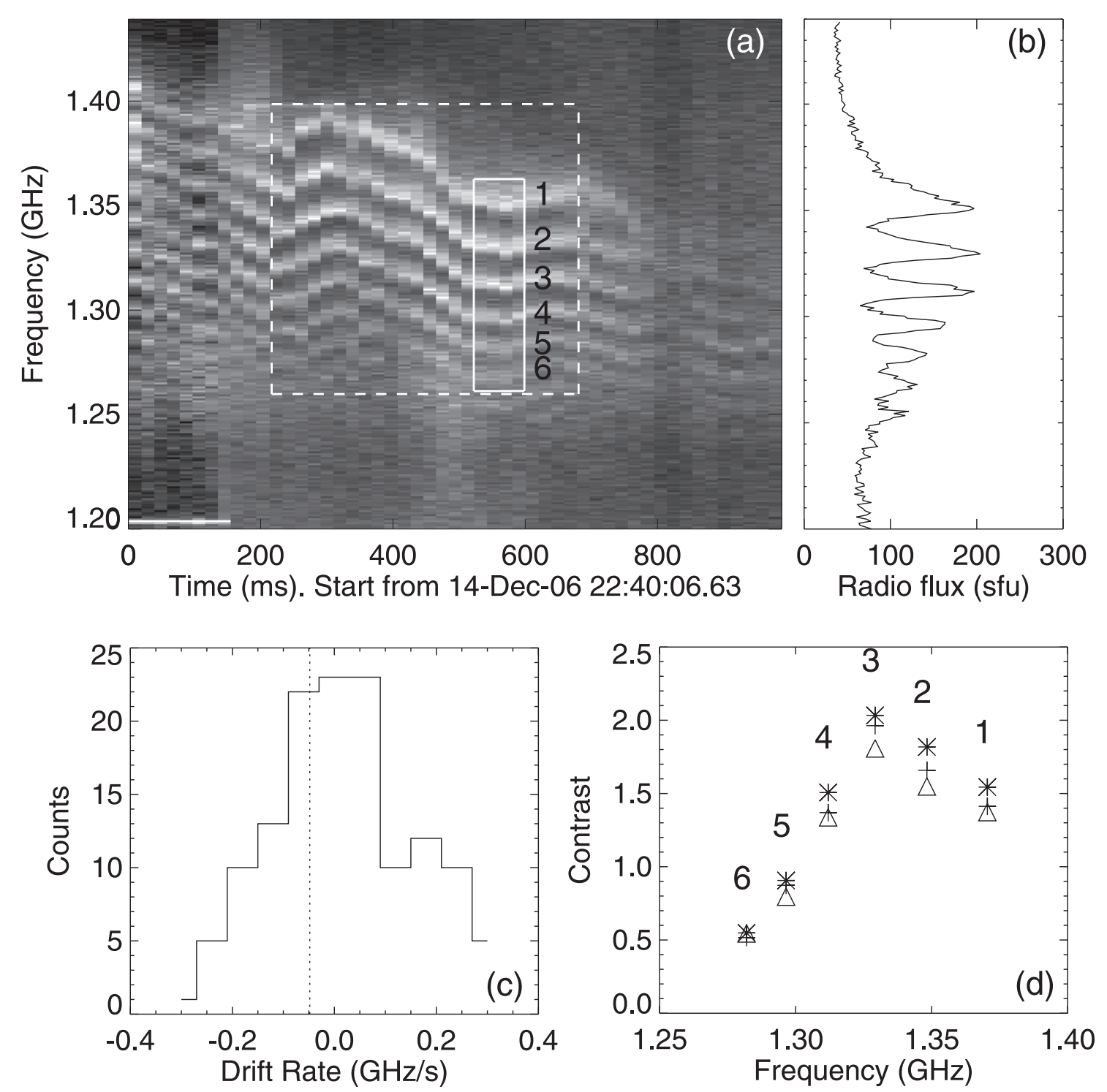

Fig. 2.6.- (a): A zebra-pattern structure observed at around 22:40 UT on 2006 December 14. Six successive strong stripes with decreasing frequencies are marked by numbers 1 to 6 . (b): Frequency profile of zebra-pattern structure, averaged in the time denoted by the small solid box (between 22:40:07.18 UT and 22:40:07.26 UT). (c): Histogram of drift rates $d \nu / d t$ of zebra stripes in the large dashed box. Vertical line indicates the mean drift rate is about $-50 \mathrm{MHz} \mathrm{s}^{-1}$. (d): Intensity contrasts $P=\left(I_{\mathrm{on}}-I_{\mathrm{off}}\right) / I_{\mathrm{off}}$ at the six zebra stripes. Pluses, stars, and triangles denote respective contrasts of baselines $5-6,6-7$, and $7-5$. 
red. One can clearly see that $\Delta \nu_{s} / \nu_{m}$ increases with frequency. This phenomenon is commonly seen in zebra patterns reported by other authors in both the meter and decimeter wavelength range (e.g., Chernov, 2005; Chernov et al., 2005).

\section{Apparent ZP Source Size}

With only three antenna baselines it is not possible to image the ZP source. However, we are able to constrain the source size using the visibility amplitudes as a function of antenna baseline. We assume that we can characterize the source brightness distribution as a symmetrical Gaussian. The visibility function is then likewise a Gaussian and a simple model, characterized by a single spatial scale, can be fit to the normalized visibility amplitudes as a function of spatial frequency. We have fit the source at frequencies "on" the bright zebra stripes (hereafter "on-stripe source") and at frequencies "off" the zebra stripes; that is, in between the zebra stripes (hereafter "off-stripe source"). As is shown in Figure 2.7(a), a simple Gaussian model adequately fits both the on- and off-stripe sources. The visibility amplitudes of the three baselines have been normalized by the total power measured by each antenna. Figure 2.7(b) shows the corresponding Gaussian FWHMs of the on- and off-stripe sources in the spatial domain for the six zebra stripes in Figure 2.6(a). They both have values near $50^{\prime \prime}$, but the sizes of the off-stripe sources are systematically larger than those of the on-stripe sources by $\approx 10^{\prime \prime}$, except for the stripes at lower frequencies where the contrast is low (see Figure 2.6(d)), a point to which we return in Section 2.3.1.

The uncertainty of the source size estimation depends on the accuracy of normalized amplitude measurements. Within the context of the model a difference of $20 \%$ in the inferred FWHM of the on- and off-stripe sources implies that the normalized amplitude of the off-stripe source on the longest antenna baseline (antennas 6-7) is 

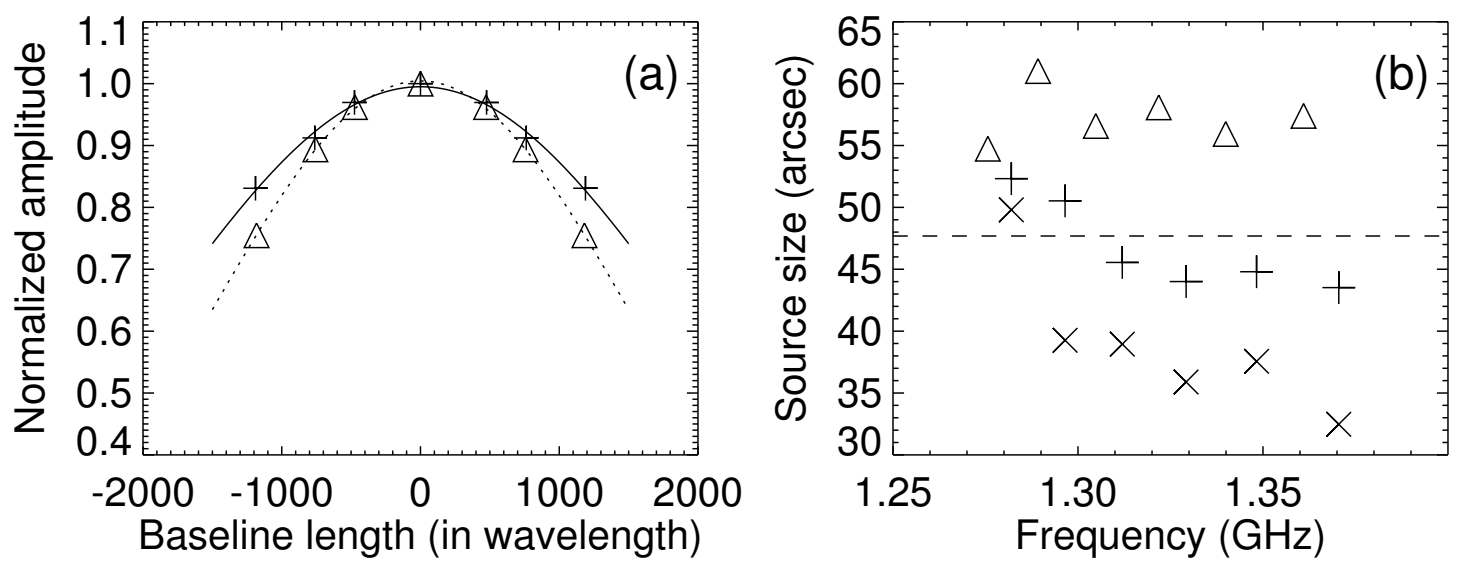

Fig. 2.7.- (a) An example of Gaussian fits of the relative visibility amplitudes on one zebra stripe. Pluses and triangles denote the on- and off-stripe sources respectively. The seven data points for each fit are the relative visibility amplitudes for the three FST baselines (both positive and negative, plus one total power/zero-spacing amplitude). A narrower Gaussian in this visibility plot implies a spatially larger source. (b) Source size estimations on the six zebra stripes in Figure 2.6(a). The " $\times$ " symbols denote the zebra source sizes after removing the contribution from the continuum (off-stripe) source. The average value of the on- and off-stripe source sizes is given by the horizontal dashed line. 
lower than that of the on-stripe source by $\gtrsim 10 \%$. The normalized amplitude measurements used in our fitting are averaged values along each zebra stripe. Therefore, the systematic errors can be represented by the statistical standard deviation of the mean of the sample, which are respectively $\lesssim 1 \%$ and $\lesssim 2 \%$ for the on- and off-stripe sources. We therefore regard errors as large as $10 \%$ as unlikely and conclude that the on-stripe source is marginally more compact than the off-stripe source. Qualitatively similar results were obtained by Chernov et al. (1994) using one-dimensional NRH observations at a much lower frequency of $164 \mathrm{MHz}$.

Note that both sources are likely strongly affected by scattering. Bastian (1994a) showed that scattering by the overlying inhomogeneous corona can play an important role in modifying the angular structure of the emission source at wavelengths longer than a few centimeters. The estimated source sizes of $\approx 50^{\prime \prime}$ are consistent with angular scattering and the intrinsic source sizes could be significantly smaller. Given an apparent source size of $50^{\prime \prime}$, the lower limit of the brightness temperature of ZP source can be estimated to be $\approx 10^{9} \mathrm{~K}$.

\section{Relative Locations of the ZP and Continuum Sources}

We now ask whether the on- and off-stripe emissions originate from two different source locations. If they are indeed separated from each other spatially, how are they related to each other? Second, how do the source locations vary with time? Finally, do the on- and off-stripe source locations have any significant dependence on frequency? In other words, does the radiation at different frequencies come from different spatial locations or not?

The interferometric phase embodies the spatial information of the radiation source. We first consider the "dynamic phase spectrum" of the ZP emission for the three base- 
lines to gain a qualitative impression (Figure 2.8). For baselines 5-6 and 6-7, zebra stripes can be distinguished as darker colors compared with the background continuum. This means that the on- and off-stripe phases are measurably different. In addition, the phase difference seems larger in the upper left region (higher frequencies and earlier times) than later in the event. For baseline 7-5, however, no phase differences are evident between on-stripe and off-stripe emissions.

In order to characterize on- and off-stripe phases quantitatively, we employ "phasor diagrams". In a phasor diagram, the amplitude and phase of each measurement are displayed in a polar plot. We have made phase measurements for both the on- and off-stripe emissions of the spectral fragment indicated by the dashed box in Figure 2.6(a), which includes six zebra stripes in an interval of $0.48 \mathrm{~s}$ with 24 consecutive observations (from 22:40:06.86 UT to 22:40:07.34 UT). The rms phase noise of a single data point (duration $20 \mathrm{~ms}$ and bandwidth roughly $1 \mathrm{MHz}$ ) is about $5^{\circ}$, too large for tracking the phase variations in time and frequency among individual measurements. To increase the signal-to-noise ratio we averaged the data as follows. First, we obtained the on- and off-stripe phases by averaging the three frequencies about the flux maximum/minimum at each time. Second, we considered averages of the phases "along" the on- and off-stripe positions (in time) and "across" the six on- and off-stripe positions (in frequency). By averaging along the zebra stripes, we constrain the phase variations of the on- and off-stripe sources as a function of frequency, so that the spatial distribution of the ZP source over frequency can be revealed. Implicit in this treatment is the assumption that the $\mathrm{ZP}$ source is likely to maintain the same structure as a function of frequency during the brief averaging time. Similarly, by averaging the on- and off-stripe phases in frequency, we track the phase variation of the ZP source with time, so that the evolution of the source centroid location with 

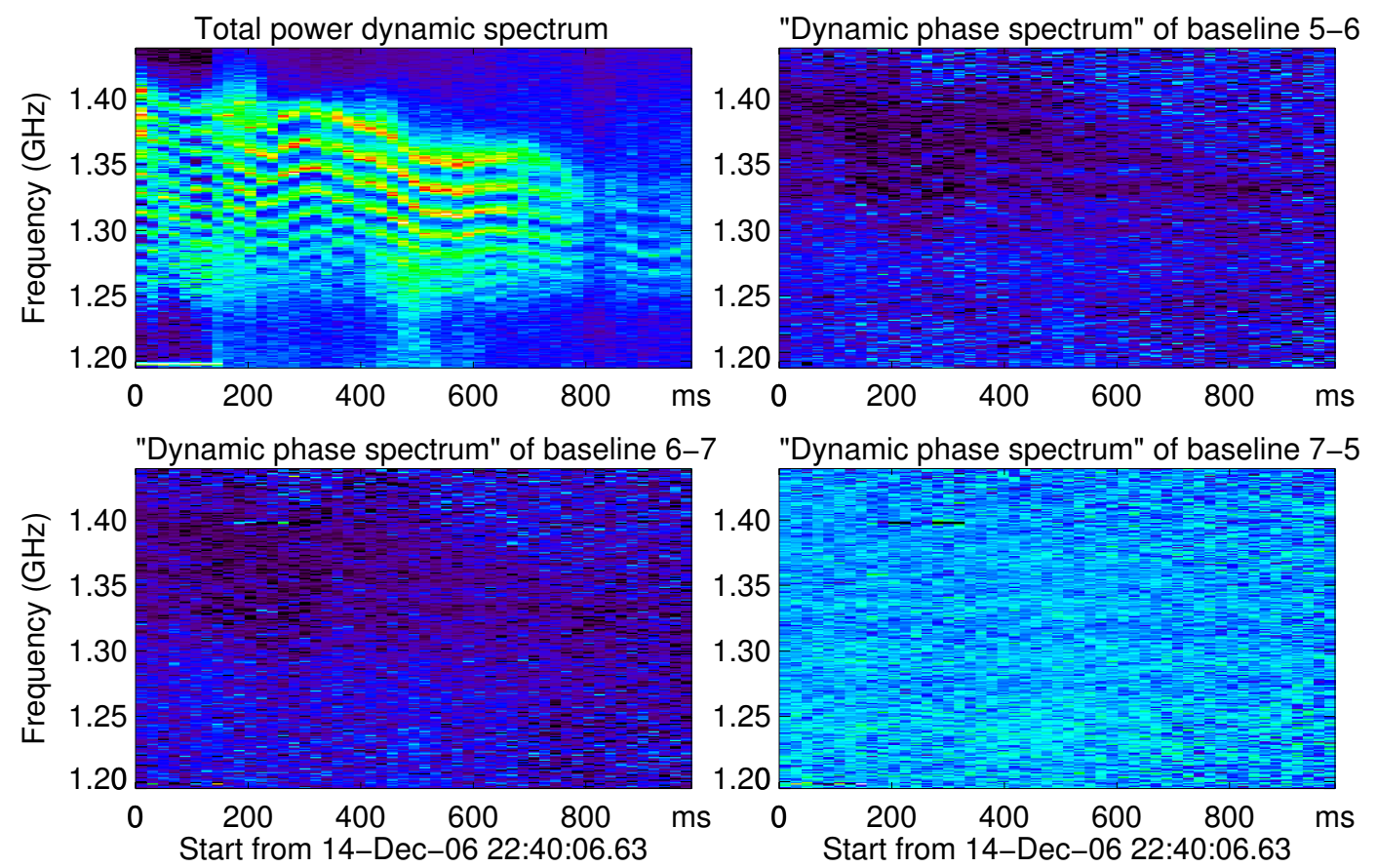

Fig. 2.8. - The total power dynamic spectrum as well as the "dynamic phase spectrum" of the three baselines. For baseline 5-6 and 6-7 the phases at the zebra stripes (darker colors) and background continuum are evidently different, but there is no notable difference for baseline 7-5. 
time can be seen, considering that different stripes of the ZP show the same drift motion according to the dynamic spectra. This approach allowed us to reduce the statistical phase error by a factor of several and to show systematic variations in the on- and off-stripe phases in the phasor diagrams with increased signal-to-noise ratio.

Figure 2.9 shows the results for the on-stripe (pluses) and off-stripe (triangles) emissions after averaging across the six zebra stripes. The pluses and triangles are colored from black to red to indicate the variation of amplitudes and phases in time. The dashed lines in each panel represent increments of $5^{\circ}$ in phase. It can be seen that the on-stripe phases of baselines $5-6$ and $6-7$ drift by $\approx 9^{\circ}$ within $0.48 \mathrm{~s}$ toward the offstripe phases, while the off-stripe phases show no evident drift. No significant phase drift is seen for baseline $7-5$, thus the direction of the spatial drift is nearly along the 7-5 interferometric fringe, which is NE-SW at the time of observation. By applying the fringe spacings (which are respectively $274^{\prime \prime}, 176^{\prime \prime}$, and $439^{\prime \prime}$ for baselines $5-6$, 6-7, and 7-5 at the time of observation) and orientations of the three FST baselines (see Figure 2.4), this amount of phase drift in time can be translated into a spatial drift of $15.6 \pm 6^{\prime \prime} .5$ from NE to $\mathrm{SW}$ on the solar disk, corresponding to a projected drift velocity of $2.5 \pm 1.0 \times 10^{9} \mathrm{~cm} \mathrm{~s}^{-1}(\approx 0.1 c)$. The estimated spatial error is based on the rms error of the FST phase measurements $\left(\approx 5^{\circ}\right)$, the total number of data points averaged, and triangulation of the three fringes. The fringe orientations of the three baselines yield the error to be orientationally dependent, which is larger in the NE-SW direction than the SE-NW direction.

In addition, there is an evident difference in mean phase of about $6^{\circ}$ between the on- and off-stripe emissions for baselines 5-6 and 6-7, shown by the arrows in Figure 2.9. That means the on- and off-stripe emissions are separated in space by an average of $8 \pm 0^{\prime \prime} .9$ in the NE-SW direction. 

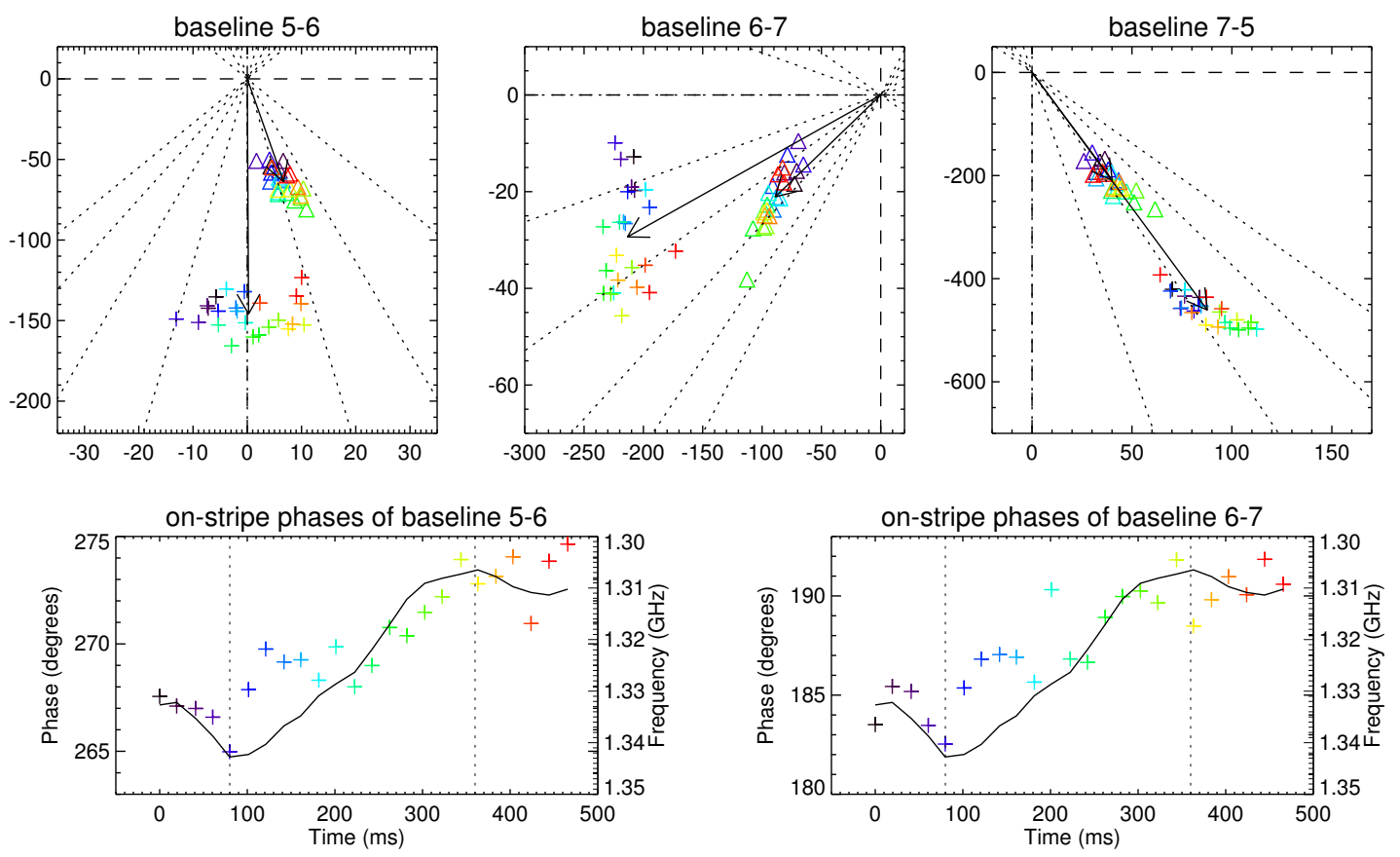

Fig. 2.9.- First row: phasor diagrams showing the visibility amplitude and phase variations of $\mathrm{ZP}$ for all the three baselines. The visibilities are averaged across the zebra stripes, showing the variations in time (colored from black to red). The amplitude and phase for each visibility data are represented by its absolute distance from the origin and the direction, respectively. Pluses and triangles denote the on- and off-stripe sources. Arrows give the average amplitudes and phases. Second row: ZP phases of baselines 5-6 and 6-7 as a function of time. The solid line, repeated in each panel, represents the ZP frequency as a function of time (note that the frequency scale on the right axis is reversed-with frequency decreasing from high to low values). 
In the two bottom panels of Figure 2.9 the ZP phases shown in the top left and top middle panels (baselines 5-6 and 6-7) are plotted as a function of time. The solid line, repeated in each panel, represents the ZP frequency as a function of time (note that the frequency scale on the right axis is reversed - with frequency decreasing from high to low values). Although the variations in time are irregular, there is a rather good correlation between the phase and the frequency; stated another way, there is a good correlation between the ZP source centroid position and the mean ZP frequency.

Figure 2.10 shows similar phasor diagrams. Here, however, the data are averaged along the zebra stripes, instead of across the zebra stripes as above, and the amplitude and phase variations are shown as a function of frequency. The data are color-coded from black to red for stripes with decreasing frequencies. The pluses and triangles denote the on- and off-stripes sources, respectively. The amplitudes of the off-stripe source barely change, but those of the on-stripe source drop with decreasing frequency. There is still little change in phase for baseline 7-5, but both the on- and off-stripe phases shift by several degrees monotonically across the six stripes. The shifts of onstripe phases seem to be slightly larger than those of the off-stripe phases. Therefore, it appears that the source centroid positions of both the on- and off-stripe sources show a systematic displacement with frequency, which is respectively $14.6 \pm 2^{\prime \prime} .8$ and $8.3 \pm 2^{\prime \prime} .8$ across the six zebra stripes from NE to SW.

The spatial difference between the on-stripe and off-stripe emissions demonstrates that there are indeed two spatially separated sources that contribute to the event, namely, the zebra-stripe emission and the background continuum source. This, too, has been noted in previous examples of ZP events using one-dimensional NRH observations at much lower frequencies (Chernov, 2006, and references therein). Care must be taken in further interpretation of the phasor diagrams. The off-stripe emis- 

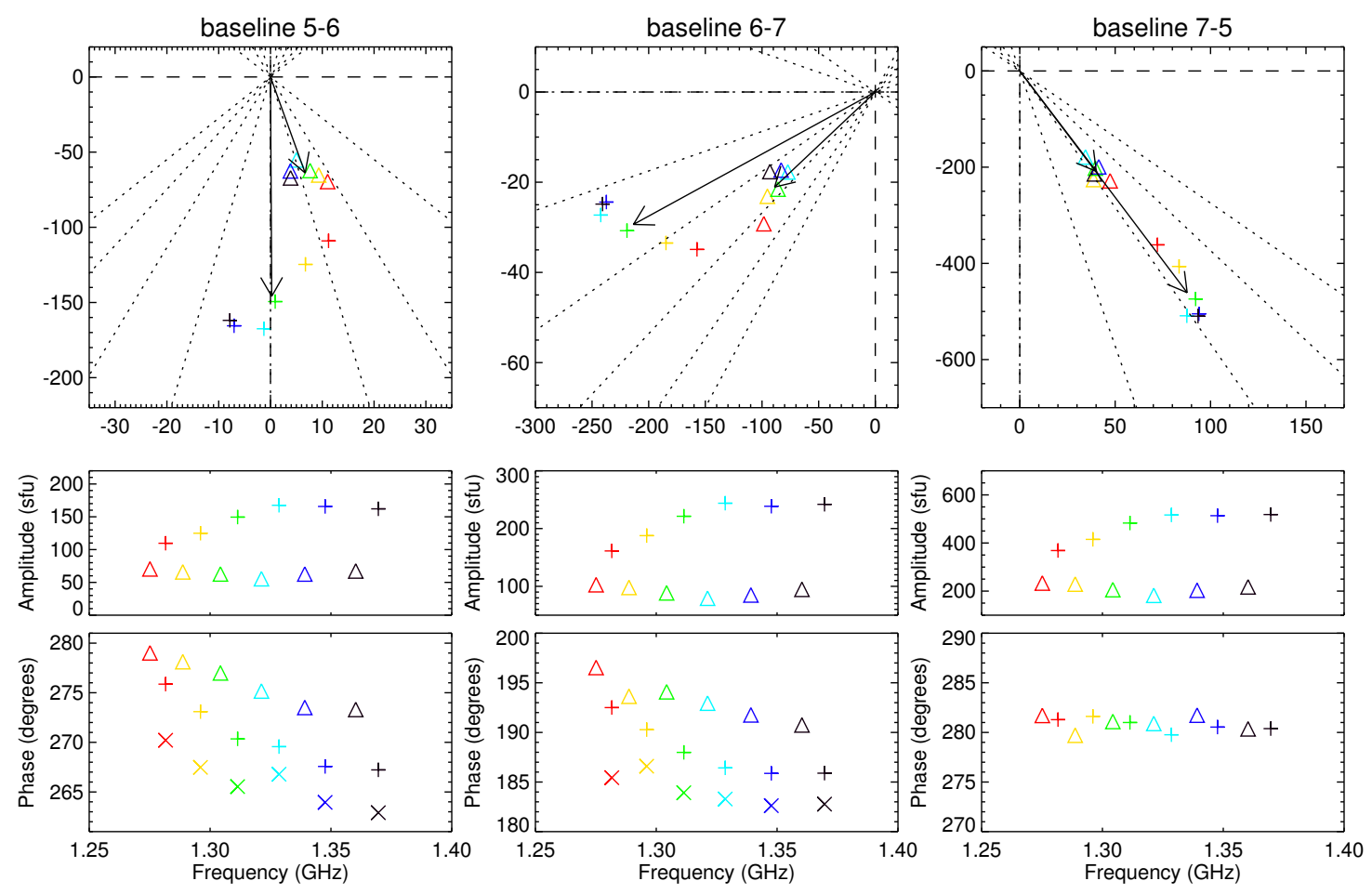

Fig. 2.10.- First row: phasor diagrams showing the visibility amplitude and phase variations of $\mathrm{ZP}$ for all the three baselines. The visibilities are averaged along the zebra stripes, showing the variations in frequency (colored from black to red for the six stripes with decreasing frequency). Pluses and triangles denote the on- and off-stripe sources. Arrows give the average amplitudes and phases. The second and third rows give the visibility amplitudes and phases of the six stripes. In the first and second panels of the third row, the " $\times$ " symbols denote the corrected phases of the zebra source. 
sion is dominated by the continuum source. However, the on-stripe emission has contributions from both the continuum and ZP sources. Therefore, a change of relative intensities of the zebra and continuum sources can result in an apparent position shift of the emission centroid measured at on-stripe frequencies. The phase drift of the on-stripe emission in time (Figure 2.9) is not in question in this respect because the amplitudes barely change in time along a given stripe. However, there is an obvious change of on-stripe amplitude with frequency at a given time - the amplitude decreases from high to low frequencies and the phase tends toward the value of the off-stripe emission (Figure 2.10), which could be caused by the intensity modulation itself. In order to correct for this effect, we vector-subtract the complex amplitudes of the off-stripe source from those of the on-stripe source in the phasor diagram, and plot the actual phases of the zebra source as " $\times$ " symbols in the first and second panels of the bottom row in Figure 2.10. After the correction, the ZP phases of the baseline 5-6 and 6-7 still show monotonic displacements as a function of frequency, but now they become comparable to those of the continuum (off-stripe) phases. Again converting to angular displacements, the corrected ZP source centroid has a displacement of $8.5 \pm 2^{\prime \prime} .8$ across the six zebra stripes with a direction from NE to SW, which is smaller than the value we obtained previously for the on-stripe source (14.6 $\left.\pm 2^{\prime \prime} .8\right)$ but comparable to that of the continuum (off-stripe) source $\left(8.3 \pm 2^{\prime \prime} .8\right)$.

The variation in the on-stripe source sizes seen in Figure 2.7(b) can also be explained by the variation of intensity contrast. The off-stripe source sizes are nearly unaffected by the contrast variation because the continuum source continuously dominates the emission at off-stripe frequencies. But since the on-stripe emission is a mixture of zebra and continuum emission, the variation in contrast plays an important role. At high contrasts (e.g., $P \geqslant 1.5$ at the number $1-4$ stripes), the zebra 
source dominates the on-stripe emission, and the source size measurements primarily reflect the property of the zebra source. At lower contrasts (e.g., $P \leqslant 1$ at the number 5-6 stripes), the contribution from the continuum source becomes increasingly important and the source size measurements start to reflect the property of the continuum source. Therefore, the on-stripe source sizes start to increase at lower-frequency zebra stripes with decreasing intensity contrasts, and reach the size of the off-stripe source, as shown in Figure 2.7(b). If we do the vector subtraction on the on-stripe source as above, the corrected relative intensities of zebra source can be obtained, hence their sizes can be fitted accordingly and plotted in Figure 2.7(b) as " $\times$ " symbols. An up to $20^{\prime \prime}$ difference between the zebra and continuum (off-stripe) source sizes can be seen after the correction (note the fitted value of the lowest frequency stripe is unreliable, because its relative intensities have large errors that comes from the subtraction of two sources with comparable intensities). We suggest that the size difference is real and indicates the zebra source is yet more compact $\left(35^{\prime \prime}-40^{\prime \prime}\right)$ than the source size deduced in Section 2.3.1 $\left(45^{\prime \prime}-50^{\prime \prime}\right)$ and the lower limit to the ZP brightness temperature should therefore be increased by $\approx 60 \%$ to $1.6 \times 10^{9} \mathrm{~K}$.

\section{Absolute ZP Source Location}

Once calibration of the FST was achieved, as described in Section 2.2, we could locate the absolute source positions by using the three interferometric fringes corresponding to the three antenna baselines to triangulate. The averaged location (in time and frequency) of the ZP source from 22:40:06.86 to 22:40:07.34 UT on the solar disk is shown in Figure 2.4 as the intersection of the three fringes, with a dashed circle representing the apparent source size of $\approx 50^{\prime \prime}$. The error of the source location is between $1^{\prime \prime} .3$ and $3^{\prime \prime} .5$ depending on direction. The ZP source is located $\approx 100^{\prime \prime}$ to 
the west of the main flaring emission. For comparison, the OVSA 4.6-6.2 GHz map at the same time is over-plotted as contours (the levels have an increment of $10 \%$ of the maximum), showing that the higher-frequency emission is well-correlated with the three Ca II H bright regions (Figure 2.4(b)). The 4.6-6.2 GHz source is far less intense than the ZP emission (cf. Figure 2.3) and is due to incoherent, non-thermal gyrosynchrotron emission.

The variation of relative source centroid locations in time and frequency, as was discussed in Section 2.3.1, can be now seen from the change of absolute locations on the solar disk. The variation of source location along the zebra stripes - that is, from 22:40:06.86 to 22:40:07.34 UT - is shown in Figure 2.11(a). The pluses denote the onstripe source locations, with the connecting arrow representing the projected spatial drift of $15^{\prime \prime} .6$ from NE to SW in $0.48 \mathrm{~s}$. The error bar in the lower left corner gives the estimated error of the on-stripe location. The off-stripe continuum source location is denoted by a single triangle because it does not display a significant drift in time, with the error bar plotted in the lower right corner. Figure 2.11(b) shows the source locations as a function of frequency across six zebra stripes. The on- and off-stripe source locations from stripes numbered 1 to 6 (decreasing in frequency) in Figure 2.6(a) are marked by the pluses and triangles. The " $\times$ " symbols show the "actual" locations of the zebra source after removing the effect of relative intensity variations. The error is denoted by the error bar in the lower left corner. The connecting arrows show the variation of the source locations with decreasing frequency. We can see that as the frequency decreases, the zebra and continuum (off-stripe) source locations both shift from NE to SW by about $8^{\prime \prime}-9^{\prime \prime}$, and they are separated from each other by $\approx 11^{\prime \prime}$. 

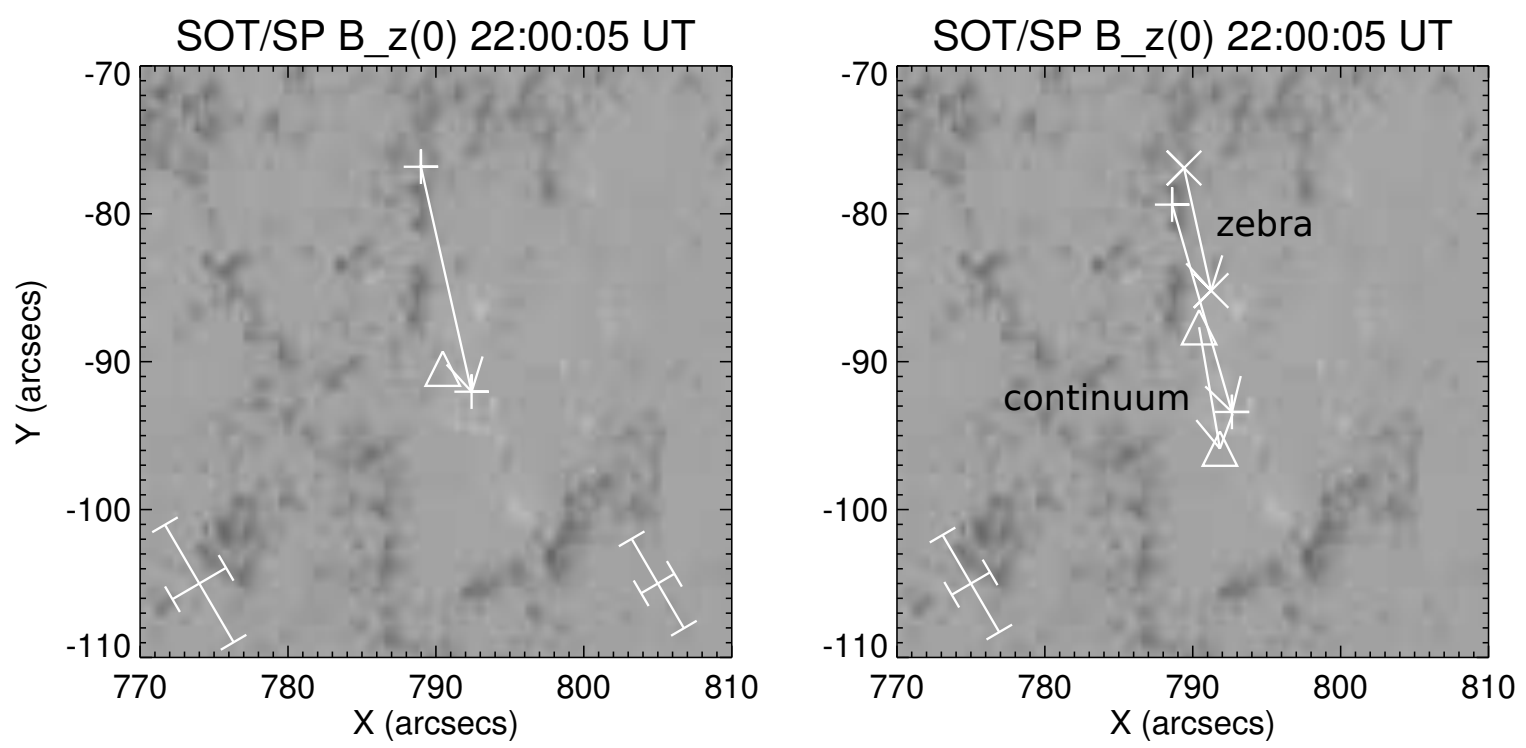

Fig. 2.11.- (a) The variation of source centroid location along the zebra stripes (with time). The pluses denote the on-stripe source locations along the zebra stripes from 22:40:06.86 to 22:40:07.34 UT (marked in the dashed box in Figure 2.6(a)), and the arrow shows the position drift. The error is represented by the error bar in the lower left corner. The triangle denote the off-stripe (continuum) source location, which shows no evident drift, with the error bar plotted in the lower-right corner. (b) The variation of source centroid location across the six zebra stripes (with frequency). The pluses, triangles, and the " $\times$ " symbol denote respectively the on-stripe, off-stripe, and corrected zebra source locations from stripe number 1 to 6 in Figure 2.6(a) (decreasing in frequency). The arrows give their corresponding position displacements. The error bar in the lower left corner gives the error. 


\subsubsection{Magnetic Field Configuration}

We are interested in constraining the location of the ZP source within the threedimensional magnetic field configuration. We performed a nonlinear force-free field (NLFFF) extrapolation based on the photospheric vector magnetogram obtained by the SOT/SP. The SOT/SP measures Stokes profiles of two magnetically sensitive Fe lines at 630.15 and $630.25 \mathrm{~nm}$. We started from the SOT/SP level 2 data that are inverted using the "MERLIN" inversion code from the polarization spectra. The $180^{\circ}$ azimuthal ambiguity in the transverse magnetogram was resolved using the "minimum energy" method (Metcalf et al., 2006). The observed vector magnetogram in the image plane (in heliocentric coordinates) was then transformed to heliographic Cartesian coordinates. Because the photospheric magnetic field is not necessarily force-free, an assumption inherent to NLFFF extrapolation as a boundary condition, and since the measurements contain inconsistencies and noise, the measured photospheric magnetic field was preprocessed to mitigate these effects. Here we used the preprocessing method developed by Wiegelmann et al. (2006). Finally, we performed the NLFFF extrapolation using the resulting magnetogram in the heliographic Cartesian coordinates using the weighted optimization method (Wiegelmann, 2004), which is an implementation of the original method of Wheatland et al. (2000). Best results for a NLFFF extrapolation are achieved when positive and negative magnetic flux are balanced; the FOV we selected to perform the extrapolation is therefore $244^{\prime \prime} \times 163^{\prime \prime}$, centered on the active region. The extrapolation was then calculated on a $240 \times 160 \times 160$ grid with a resolution of $1^{\prime \prime} .02$ in the $X$-, $Y$ - and $Z$-directions (corresponding to the directions of west, north, and normal to the tangent plane centered on the active region).

In Figure 2.4, we have already seen that the location of the ZP source is nearly 

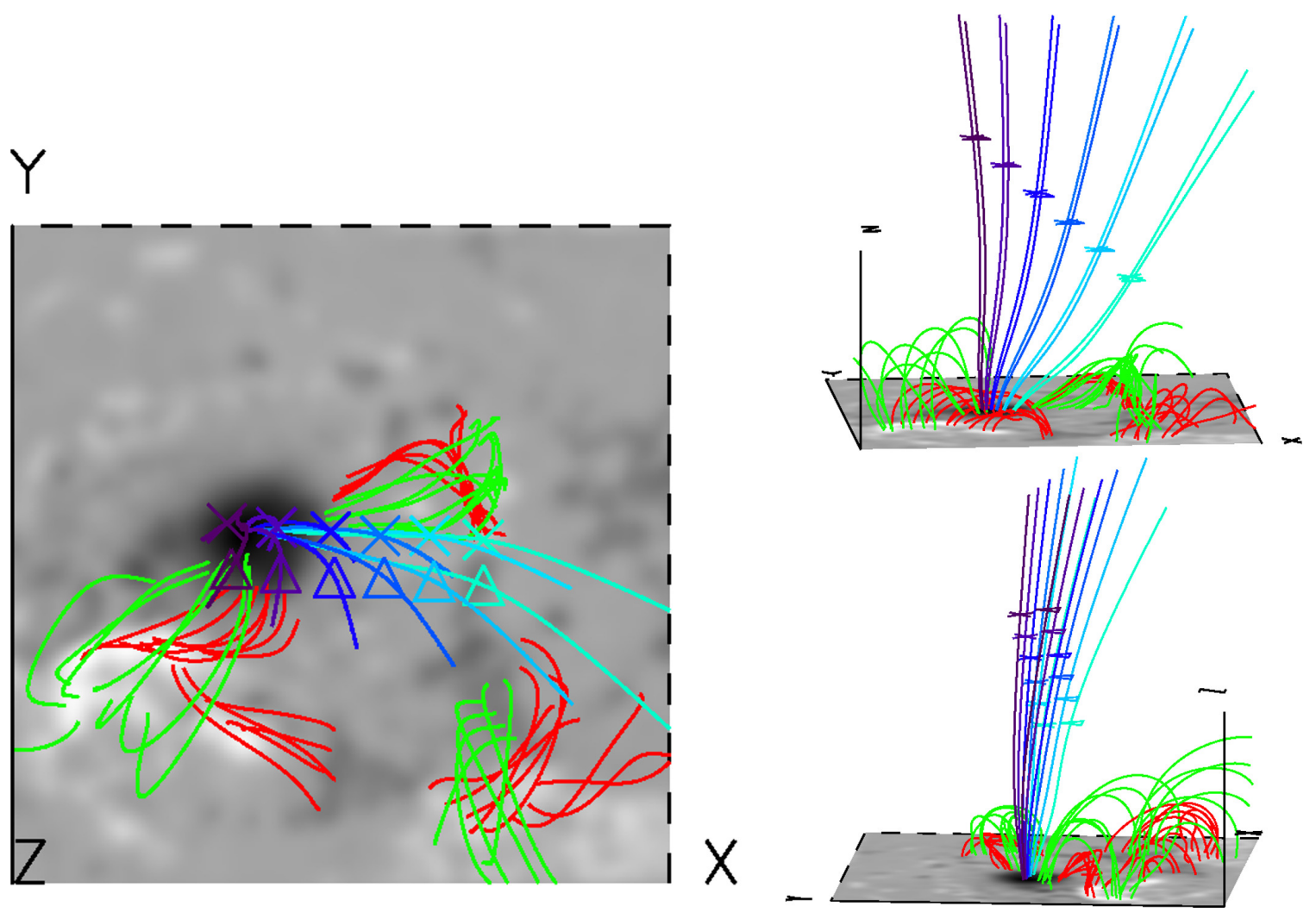

Fig. 2.12.- Deprojected zebra and continuum source locations with the NLFFF extrapolation field lines. The left image is the view from top, while the two images on the right are for side views from south and east (the $X$ - and $Y$-axes point at west and north, and the $Z$-axis is perpendicular to the tangent plane centered at the active region). The " $\times$ " symbols and triangles denote a series of possible three-dimensional locations of continuum and zebra sources consistent with their projected locations (averaged in time and frequency) on the image plane. The extrapolated field lines in blue colors passing the possible radio source locations are probably the post-flare loops in which the radio source is located. The colors from light to dark blue denote the magnetic field strengths from 90 to 30 Gauss at heights from 40 to $80 \mathrm{Mm}$. The magnetic configurations of the three major Ca II H bright regions (see Figure 2.4(b)) are also shown by the extrapolated field lines, which are grouped and colored in red and green to represent increasing coronal heights. 
$100^{\prime \prime}$ away from the active region center in the image plane. Since the active region is at S06W46, the ZP source is apparently located relatively high in the solar corona above the active region. The projected ZP source location is known but the location along the line of sight is unknown. We obtained a series of possible three-dimensional locations of the radio source, consistent with its projected location, and plot them in Figure 2.12. The zebra and continuum source locations (averaged in time and frequency) are marked as " $\times$ " symbols and triangles respectively, and color-coded in magnetic field strength (from light to dark blue, the magnetic field changes from $\approx 90$ $\mathrm{G}$ to $\approx 30 \mathrm{G}$ at coronal heights from $\approx 40 \mathrm{Mm}$ to $\approx 80 \mathrm{Mm})$. It can be seen that as the radio source locations are placed higher in the corona, the magnetic field strength decreases, and they move to a position more nearly over the large sunspot with negative polarity. A group of extrapolated field lines in blue colors is drawn passing these possible source locations, showing a post-flare loop system that is connected with the large sunspot with negative polarity. The polarity of these field lines suggest that since the $\mathrm{ZP}$ is $\mathrm{RCP}$, it is polarized in the sense of the ordinary mode. We note, too, that a Ca II H brightening pointed out at the beginning of Section 2.3 (in Figure 2.5(c)) is near the footpoints of the post-flare loops in which the ZP source may be located. These field lines have orientations from NE to SW and extend to large coronal heights. For completeness, we also show the extrapolated field lines of the three major Ca II H bright regions (see Figure 2.4(b)), which are grouped and colored in red and green to represent increasing coronal heights.

\subsection{Discussion}

The key findings of the previous section may be summarized as follows: 
- An intense X1.5 flare was observed on 2006 December 14. The flare was accompanied by intense, prolonged, and variable decimeter wavelength emission as well as gyrosynchrotron emission at centimeter wavelengths. A striking ZP radio burst was observed during the decay phase, centered near $1.32 \mathrm{GHz}$, with an overall frequency bandwidth of up to $150 \mathrm{MHz}$. It is completely RCP and consists of up to 12 zebra stripes superposed on broadband continuum emission. The stripes drift in the spectrum irregularly as an entity with an average drift rate of $-50 \mathrm{MHz} \mathrm{s}^{-1}$. The relative frequency separations $\Delta \nu_{s} / \nu_{m}$ between adjacent stripes are $\approx 1.3 \%$, with $\Delta \nu_{s} / \nu_{m}$ increasing with frequency.

- Two radio sources with a spatial separation of $\approx 11^{\prime \prime}$ —zebra and background continuum - contribute to the ZP emission. The apparent zebra source size is around $35^{\prime \prime}$, which is systematically smaller than that of the continuum source by as much as $20^{\prime \prime}$, after the correction for the relative intensity effect. The source size of both the ZP and continuum sources are likely strongly affected by scattering. The lower limit of the brightness temperature of ZP is estimated to be $1.6 \times 10^{9} \mathrm{~K}$.

- The zebra source centroid drifts irregularly in time with an average drift of $\approx 16^{\prime \prime}$ in 0.48 s from NE to SW (corresponding to a projected average velocity of $2.5 \times 10^{9} \mathrm{~cm} \mathrm{~s}^{-1}$ ), while the continuum locations show no evident drift with time.

- Both the ZP on-stripe and off-stripe source locations are frequency dependent. After correction of the source centroid positions for relative intensity variations, the centroid position shifts $8^{\prime \prime}-9^{\prime \prime}$ for both the zebra and continuum sources in the direction from NE to SW across the six stripes from high to low frequency 
at a fixed time.

- The zebra and continuum sources are possibly located in a post-flare loop system with an orientation from NE to SW, which connects to the large sunspot with negative polarity. The polarity of the magnetic field and the observed sense of circular polarization of the ZP imply that it is $o$-mode.

Of these findings, perhaps the most significant are that the ZP (on-stripe) and continuum (off-stripe) emissions show an angular separation as well as a difference in source size; and that the ZP and continuum source locations are frequency dependent. In other words, the radiation at different frequencies originates from different spatial locations. We are able to conclude that the ZP and continuum sources are not spatially coincident and that both the ZP and continuum sources are spatially extended.

We should point out that the spatial displacement of the on- and off-stripe emissions suggests that the previous measurements of ZP spatial drift in time based on interferometric observations at a single frequency may be misleading. Since the ZP shows frequency drifts with time as an entity, a record of the flux density with time at a single frequency cuts through different zebra stripes as well as the off-stripe continuum source. Apparent spatial shifts can appear as a result of the switch between different sources with different locations. We note that Altyntsev et al. (2005) reported a microwave zebra-pattern structure near $5.7 \mathrm{GHz}$ that was observed on 2003 January 5 by the $S B R S$ and the $S S R T$. The apparent source size, measured at a frequency far less influenced by scattering, was measured to be $<10^{\prime \prime}$. The source positions of two successive ZP stripes coincide spatially in the east-west direction. For these reasons, the authors concluded that the emission is due to the nonlinear coupling of Bernstein waves in an unresolved source. Yet in Altyntsev et al. (2005) 
the spatial coincidence of the two ZP stripes was based on measurements in one dimension at a fixed frequency at different times. It is possible that the ZP source location drifting with time can result in the apparent "coincidence" of the two stripes. Moreover, even if the two stripes do coincide spatially in the east-west direction, there could be an unknown separation in the north-south direction that is not revealed by the one-dimensional measurements.

In the case of the 2006 December 14 ZP event reported here, and in contrast to the observations of Altyntsev et al. (2005), there is clear evidence that the source location is frequency dependent. Taken together with the theoretical difficulties raised by various authors (e.g., Zlotnik, 2009), we conclude that Bernstein models are unlikely to be relevant to this $\mathrm{ZP}$ emission and we turn our attention to whistler and DPR models.

The observed projected average spatial drift velocity of the ZP source along the zebra stripes $\left(2.5 \times 10^{9} \mathrm{~cm} \mathrm{~s}^{-1}\right.$ in the NE-SW direction) is about 40 times larger than the local Alfvén velocity of $\approx 7 \times 10^{7} \mathrm{~cm} \mathrm{~s}^{-1}$ calculated as $V_{A}=B /\left(4 \pi n_{i} m_{i}\right)^{1 / 2} \approx$ $2 \times 10^{11} B n_{e}^{-1 / 2}$, where $B$ is the magnetic strength in the radio source, assumed here to be $\sim 50 \mathrm{G}$, and $n_{e}^{1 / 2}=\nu /\left(e^{2} / \pi m_{e}\right)^{1 / 2} \approx \nu / 8980$ if one assumes the ZP emission at frequency $\nu$ is near the local plasma frequency $\nu_{\mathrm{pe}}$. This velocity is too high for most physical movements of the radio source reacting to the magnetic field variation. But it could be explained in terms of the whistler model by propagation of low-frequency whistler wave packets with group velocities $\left(v_{\mathrm{gr}}\right)$ given by (Chernov, 1976)

$$
v_{\mathrm{gr}}=2 c \frac{\nu_{\mathrm{ce}}}{\nu_{\mathrm{pe}}} \sqrt{\frac{\nu_{w}}{\nu_{\mathrm{ce}}}\left(1-\frac{\nu_{w}}{\nu_{\mathrm{ce}}}\right)^{3}}
$$

in the quasi-longitudinal case, where $c$ is the speed of light and $\nu_{w}$ is the whistler wave frequency. For $\nu_{w} / \nu_{\mathrm{ce}} \approx 0.25$, the ratio at which the growth of the whistler wave is 
preferred in the decimetric range (Chernov, 2006), the group velocities can reach the observed value of $2.5 \times 10^{9} \mathrm{~cm} \mathrm{~s}^{-1}$ for $\nu_{\mathrm{pe}} / \nu_{\mathrm{ce}} \lesssim 10$ (see Figure 2 of Chernov, 1976).

Is the whistler model able to explain the spatial drift of the ZP source in frequency and time simultaneously? In the whistler model, periodically generated whistler wave packets propagate along a trajectory in the magnetic trap at the whistler group velocity $v_{\text {gr }}$. They separate regularly from each other in space so that they can emit at discrete frequencies, thereby producing zebra stripes. The frequency drift of each zebra stripe in the spectrum is determined by the motion of the corresponding whistler wave packet along its trajectory, the density gradient along its trajectory, and wavewave interactions. We suppose the observed spatial drift with time corresponds to $v_{\mathrm{gr}}$. If the coronal number density decreases exponentially with height

$$
n_{e}=n_{e 0} e^{-\Delta h / L_{n}}
$$

where $n_{e 0}$ is the plasma density at the reference height $h_{0}, \Delta h$ is the height from $h_{0}$ and $L_{n}=d h /\left(-d n_{e} / n_{e}\right)$ is the plasma density scale height, then the plasma frequency $\nu_{\text {pe }}$ can be written as

$$
\nu_{\mathrm{pe}}=\nu_{\mathrm{pe} 0} e^{-\Delta h / 2 L_{n}}
$$

The radiation is near the plasma frequency, so the frequency drift rate is

$$
\frac{d \nu}{d t}=-\nu \frac{v_{h}}{2 L_{n}}
$$

where $v_{h}$ is the vertical component of the whistler group velocity (normal to the solar surface). Given the observed average frequency drift rate $d \nu / d t \approx-50 \mathrm{MHz}$ $\mathrm{s}^{-1}$ and the projected velocity of $v_{\text {proj }}=2.5 \times 10^{9} \mathrm{~cm} \mathrm{~s}^{-1}$ (which, given its nearly $\mathrm{N}-\mathrm{S}$ orientation, is nearly parallel to the solar surface), the tangent angle of the 
trajectory is then $\tan \alpha_{1}=v_{h} / v_{\text {proj }}=3 \times 10^{-11} L_{n}$. On the other hand, we observe the projected spatial displacement from zebra stripes numbers $1-6$ to be $\Delta l_{1-6}=8^{\prime \prime} .5$, or $6.2 \times 10^{8} \mathrm{~cm}$, and their frequencies differ by $\Delta \nu_{1-6} \approx 85 \mathrm{MHz}$. It is easy to see from Equation (2.4) that the height difference between zebra stripe numbers 1-6 $\Delta h_{1-6} \approx$ $\Delta \nu_{1-6} / \nu \cdot 2 L_{n}$ for small variations of $\nu$ and $L_{n}$. Therefore, we have another estimate of the tangent angle of the whistler wave packet trajectory $\tan \alpha_{2}=\Delta h_{1-6} / \Delta l_{1-6} \approx$ $2 \times 10^{-10} L_{n}$, which is an order of magnitude larger than $\tan \alpha_{1}$ that we obtained based on the assumption that the observed source drift with time corresponded to the whistler group velocity $v_{\mathrm{gr}}$. In other words, the trajectory of whistler wave packets cannot be reconciled with both the observed spatial displacement of the ZP source with frequency and the observed frequency drift with time.

It is also worth noting that the whistler model does not account for the systematic increase of the relative spacing $\Delta \nu_{s} / \nu_{m}$ with increasing ZP frequencies as seen in Figure 2.13(a), because the spatial separation between adjacent whistler wave packets is assumed to result from the periodic injection of whistler wave packets and their frequency separation is therefore essentially constant.

We now consider whether the DPR model is consistent with the observed features of the ZP and continuum source, including both the total power spectral features and their apparent shift in spatial location as a function of time and frequency. As the ZP and continuum sources emit near the local upper-hybrid frequency $\nu_{\mathrm{uh}}$ which, with $\nu_{\text {ce }} \ll \nu_{\text {pe }}$, is near the local electron plasma frequency $\nu_{\text {pe }}$, lower-frequency emission should come from greater coronal heights for both the ZP and continuum sources. As a result, a spatial shift with frequency is expected for both the ZP and continuum sources, in accordance with the observations. Furthermore, The NE-SW direction of the spatial shift is generally consistent with the NE to SW orientation of the post-flare 
loop system in which the emission sources are located. However, the absolute height of the emission source is not yet known. The ZP stripes are those locations where $\nu_{\mathrm{uh}} \approx \nu_{\mathrm{pe}}=s \nu_{\mathrm{ce}}$ in the DPR model. We again assume an exponential dependence of coronal number density as in Equation (2.3), with a scale height $L_{n}$. The coronal magnetic field is likewise assumed to decrease exponentially with height:

$$
B=B_{0} e^{-\Delta h / L_{B}},
$$

where $B_{0}$ is the magnetic field at $h_{0}$ and $L_{B}$ is the magnetic field scale height. The electron cyclotron frequency $\nu_{\mathrm{ce}} \propto B$ and so

$$
\nu_{\mathrm{ce}}=\nu_{\mathrm{ce} 0} e^{-\Delta h / L_{B}} .
$$

It can be shown that the relative frequency spacing between the adjacent zebra stripes in the DPR model is then given by (Kuznetsov \& Tsap, 2007)

$$
\left|\frac{\Delta \nu_{s}}{\nu_{m}}\right| \simeq \frac{1}{s} \frac{1}{1-2 L_{n} / L_{B}},
$$

where $s$ is the harmonic number with $\nu=s \nu_{\mathrm{ce}}$. Given the continuity of each zebra stripe in the dynamic spectrum for the $\approx 0.5 \mathrm{~s}$ duration of the spectral fragment in our analysis (the dashed box in Figure 2.6(a)), we assume that each stripe corresponds to a single integer harmonic $s$ of the electron cyclotron frequency $\nu_{\text {ce }}$. Moreover, successive zebra stripes emitting at discrete frequencies should have a one-to-one correspondence with successive integer values of $s$, i.e., $s_{0}, s_{0}+1$, .., where $s_{0}$ is the reference harmonic number at $h_{0}$. Thus the frequency spacing $\Delta \nu_{s} / \nu_{m}$ is a function of $\left(s_{0}+i\right), \mathrm{i}=0,1,2 \ldots$. 
By using the observed values of $\Delta \nu_{s} / \nu_{m}$ for each ZP frequency at a fixed time, we obtain a pair of best-fit values of $s_{0}$ and $L_{n} / L_{B}$. In particular, for a given time, we start with a fixed $s_{0}$ and perform a least-squares fit to find $L_{n} / L_{B}$. Then we increment the value of $s_{0}$ by integer values until a minimum in the standard deviation of the fit to $\Delta \nu_{s} / \nu_{m}$ is reached. We did such fits of $\Delta \nu_{s} / \nu_{m}$ as a function of $\left(s_{0}+i\right)$ for the six zebra stripes for the 24 consecutive integrations shown by the dashed box in Figure 2.6(a). Figure 2.13(a) shows examples of these fits at five near-equally spaced times spanning the entire fitting time range (22:40:06.86 UT, 22:40:06.96 UT, 22:40:07.04 UT, 22:40:07.16 UT, and 22:40:07.26 UT), where square symbols and solid lines represent respectively the measured and best-fit values and are color-coded in time from blue to red. From the distribution of best-fit values of $s_{0}$, after excluding a few outliers, we conclude that $s_{0}=8$ is the most probable value, with a scatter of $\sigma_{s_{0}} \approx 1.4$. Therefore we assign harmonic numbers of $s=8,9,10,11,12,13$ to the six successive zebra stripes numbered from 1 to 6 in Figure 2.6(a), with a corresponding $L_{n} / L_{B} \approx 4.4 \pm 0.5$. Note that a stripe with lower frequency corresponds to a higher harmonic number, which is consistent with the previous results based on DPR models (e.g., Zlotnik et al., 2003; Kuznetsov \& Tsap, 2007). Such a ratio of the scale heights means the magnetic field changes faster than the plasma density with height, which is usually the case in the solar corona. The magnetic field strengths $B$ from stripes 1 to 6 (from low to high in height) can be estimated to be from 62 G to 35 G, by using the harmonic values $s$ and assuming $\nu \approx \nu_{\mathrm{pe}}=s \nu_{\mathrm{ce}}$. The uncertainty in $B$ can be estimated from the scatter in the distribution of $s_{0}$. We find that it ranges from $17 \%$ at $s_{0}=8$ to $11 \%$ for $s=s_{0}+5=13$. Using the magnetic fields derived from the NLFFF extrapolation results in $§ 2.3 .2$ for guidance, we suggest the zebra stripes 1 to 6 of the ZP source are consistent with a location in the post-flare loop 
system at coronal heights from 57 to $75 \mathrm{Mm}$ above the photosphere. The magnetic scale height $L_{B}$ can be estimated to be $L_{B}=d h /(-d B / B) \approx 3.2 \times 10^{9} \mathrm{~cm}$. On the other hand, an estimation of the density scale height $L_{n}$ is also available by $L_{n}=d h /(-2 d \nu / \nu) \approx 1.4 \times 10^{10} \mathrm{~cm}$, from the known frequencies of the zebra stripes (an equivalent result of $L_{n}$ can be obtained by using $L_{B}$ and the fitted value of $\left.L_{n} / L_{B} \approx 4.4\right)$.

DPR levels are formed as a result of plasma density and magnetic field variations in height, as demonstrated by Figure 2.13(b). By applying the values of $L_{n}, L_{B}$, and $s$, the dependencies of $s \nu_{\mathrm{ce}}$ and $\nu_{\mathrm{pe}}$ as a function of $\Delta h$ are given for the time denoted as the solid box in Figure 2.6(a). The reference height $h_{0}$ is set to be that of the lowest DPR level (the number 1 stripe with $s_{0}=8$ ). The intersections of the curves $s \nu_{\mathrm{ce}}(\Delta h)$ and the plasma frequency distribution $\nu_{\mathrm{pe}}(\Delta h)$ (marked by diamonds) are the DPR levels, which coincide with the observed frequencies of zebra stripes (denoted by the horizontal lines). In this event, the zebra stripes drift irregularly and rapidly in the dynamic spectrum, indicating that the DPR levels do not remain at precisely the same height in the flare loop - that is, $h_{0}$ varies by a few percent of $L_{n}$, or a few thousand kilometers - which also accounts for the scatter and temporal variation seen in Figure 2.13(a).

In the whistler model, formation of the regularly spaced stripes of ZP in frequency is based on propagation of the periodically generated whistler wave packets along a given trajectory - that is, the trajectory of each whistler wave packet determines the spatial drift of each zebra stripe in time (with tangent angle $\tan \alpha_{1}$ ), should follow the same orientation of the spatial extent of the entire ZP source, represented by the spatial displacement of zebra stripes at different frequencies (with tangent angle $\tan \alpha_{2}$ ), i.e., $\tan \alpha_{1} \approx \tan \alpha_{2}$. We have already demonstrated that they cannot 

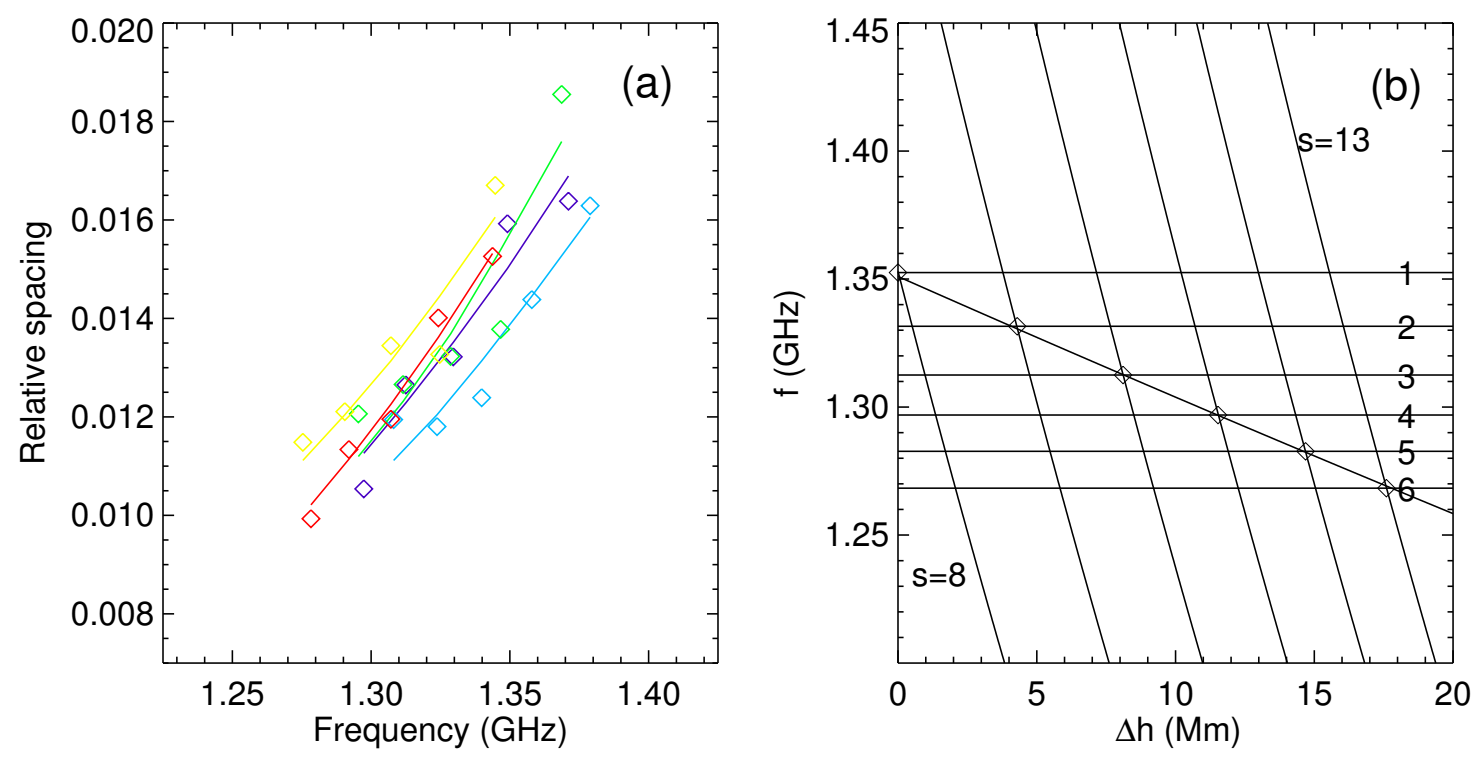

Fig. 2.13. - (a): Fitting examples of $\Delta \nu_{s} / \nu_{m}$ as a function of $\nu_{m}$ for the six zebra stripes in the dashed box of Figure 2.6(a) (which has 24 consecutive measurements in time from 22:40:06.86 UT to 22:40:07.34 UT). The square symbols and solid lines represent respectively the measured and best-fit values at five near-equally spaced times spanning the entire fitting time range (22:40:06.86 UT, 22:40:06.96 UT, 22:40:07.04 UT, 22:40:07.16 UT, and 22:40:07.26 UT). They are color-coded in time from blue to red. The most probable fitting values of the harmonic numbers are $s=8,9,10,11,12,13$ for the six zebra stripes numbered from 1 to 6 in Figure 2.6(a), and the corresponding $L_{n} / L_{B}=4.4$. (b) Horizontal lines are the averaged peak frequencies of the six zebra stripes in the solid box of Figure 2.6(a) (around 22:40:07.21 UT). The curves of gyroharmonics $s \nu_{\text {ce }}$ with $s=8-13$ are plotted as a function of coronal height $\Delta h$ relative to the height of the $s=8$ layer. The intersections of the curves $s \nu_{\text {ce }}(\Delta h)$ and the plasma frequency distribution $\nu_{\mathrm{pe}}(\Delta h)$ are the DPR levels. 
be reconciled in the context of the whistler model. For the DPR model the two "trajectories" need not coincide. The DPR levels, corresponding to the observed zebra stripes, are distributed along a coronal loop at locations where the resonant conditions are matched. At the same time, the locations of each DPR level can change with time in response to the variation of source conditions. Therefore, the "trajectory" of each DPR level is not required to follow the same orientation of the spatial distribution of the resonance layers in the loop. In fact, given the density scale height of $L_{n} \approx 1.4 \times 10^{10} \mathrm{~cm}$, we have $\alpha_{1} \approx 23^{\circ}$ and $\alpha_{2} \approx 70^{\circ}$. That means the DPR levels are distributed near vertically in the coronal loop while their apparent motion is nearly horizontal in time.

Both the ZP and continuum sources probably reside on the same post-flare/postCME loops that extend field lines from in or near the large sunspot with negative polarity to well up into the corona with an NE to SW orientation. The radio emission could be powered by an energy release site high up in the corona above the radio source. This site could be related to magnetic reconnections induced by the fast halo CME associated with this flare, which may also account for the intense and prolonged type IV burst activity in the post-flare phase, from 22:07-23:15 UT. As mentioned in $§ 2.3 .2$, the radio source may be magnetically associated with the Ca II $\mathrm{H}$ brightening feature that persists for the duration of the type IV emission. The brightening may indicate the magnetic footpoints of the field lines on which the ZP and type IV emission originates.

In the DPR model, ZPs are thought to arise from energetic electrons in a magnetic trap with a large gradient in electron momentum distribution function perpendicular to the magnetic field $\partial f / \partial p_{\perp}$, as might arise from a distribution function sharply peaked perpendicular to the magnetic field (e.g., a Dory-Guest-Harris, or ring, dis- 
tribution), or a loss-cone distribution with sufficiently narrow momentum dispersion (e.g., Winglee \& Dulk, 1986; Kuznetsov \& Tsap, 2007; Zlotnik et al., 2009). At the same time, the continuum emission can also arise from an anisotropic electron distribution in the magnetic trap, most likely, a common loss-cone distribution (e.g., Kuznetsov \& Tsap, 2007; Zlotnik et al., 2009). Therefore, it is plausible that the ZP and continuum observed by the FST is related to the injection of fast electrons that originate from the energy release site above the radio source, perhaps as the result of magnetic reconnection behind the fast halo CME associated with the flare. These downward-propagating electrons establish the anisotropic distribution that drives the zebra and continuum emission. It is highly unlikely that the ambient electron number density or the magnetic field change significantly during the $\sim 1$ s duration of the ZP event reported here and therefore cannot be the reason for variations in the ZP frequency drift with time. More likely, variations in the number and/or degree of anisotropy of the injected electrons lead to variations in the height where wave growth is favored and the DPR condition is met. As a result, the heights of the established anisotropic distribution and/or the DPR levels can be modulated, and form the irregular ZP frequency drift in the dynamic spectrum. The observed irregular drift of ZP source centroid in time can also be attributed to this effect.

As has been proposed by many authors (e.g., Winglee \& Dulk, 1986; Kuznetsov \& Tsap, 2007; Zlotnik et al., 2009), the conditions required to produce a ZP signature through the DPR instability are more stringent than those for producing a continuum, e.g., a low-momentum electron deficit, a narrower momentum dispersion, a higher overall momentum, etc. In addition, for achieving the observed large intensity contrast of the ZP to the continuum in this event, the growth rate of the ZP emission should be sufficiently higher than that of the continuum, which may require 
a higher non-equilibrium electron density. All the peculiarities mentioned above could possibly explain the observations of the relatively short-lived and fast-varying ZP and the more persistent and stable continuum in this event.

\subsection{Conclusion}

We present FST observations of a striking zebra-pattern radio burst that occurred during the 2006 December 14 X1.5 flare. This is the first observation of zebra pattern emission that combines simultaneous high spectral and time resolution data with interferometric observations over the entire bandwidth. After calibrating the FST against $O V S A$ we can obtain the absolute locations of radio fine structures on the solar disk, and study their spatial and spectral features. We conclude that the DPR model is the most favorable model since it can fit most spectral and spatial features of this ZP event.

The schematic in Figure 2.14 summarizes our interpretation of the ZP event within the framework of DPR model: the zebra and continuum source are located at a height of $60-80 \mathrm{Mm}$ in a post-flare/post-CME loop system that connects the large sunspot with negative polarity with an NE to SW orientation. Both the zebra and continuum are extended sources occupying a total height range of $\approx 20 \mathrm{Mm}$ in the post-flare loops, which explains the spatial drifts of ZP and continuum in frequency. Within the zebra source, individual stripes correspond to emissions near the local plasma frequencies at the DPR levels. We suggest that the fast electrons responsible for the continuum and $\mathrm{ZP}$ emission originate in an energy release site high up in the corona above the radio source, perhaps the result of magnetic reconnections induced by the fast halo CME. The fast and irregular spatial drift of the ZP source centroid in time and the irregular frequency drift of ZP likely result from time variations in properties of the 


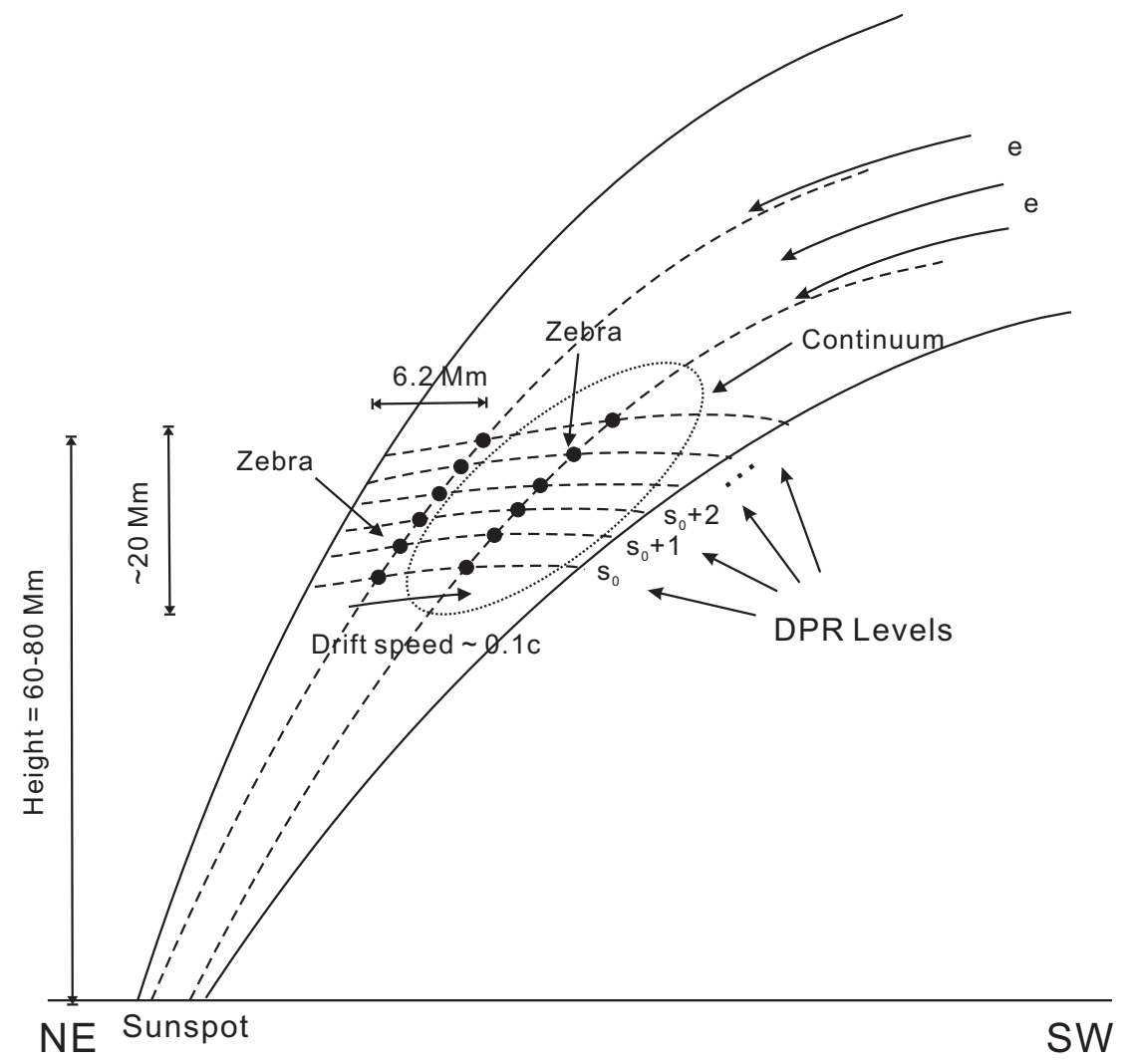

Fig. 2.14.- Simplified source model: the zebra and continuum source are located at a height of $60-80 \mathrm{Mm}$ in a post-flare/post-CME loop system that connects the large sunspot with negative polarity with an NE to SW orientation. Both the zebra and continuum are extended sources occupying a total height range of $\approx 20 \mathrm{Mm}$ in the post-flare loops. Within the zebra source, individual stripes correspond to emissions near the local plasma frequencies at the DPR levels (horizontal dashed lines, of which the lowest one corresponds to the $s_{0}=\nu_{\mathrm{pe}} / \nu_{\mathrm{ce}}=8$ layer). An energy release site is located high up in the corona above the radio source. Electron beams generated from this site propagate downward along the magnetic field lines into the magnetic trap and give rise to the instability for emission. The fast and irregular spatial drift of the $\mathrm{ZP}$ source centroid in time $(\approx 0.1 \mathrm{c}$, indicated by the arrow showing the general drift direction of $\mathrm{NE}-\mathrm{SW}$ ) and the irregular frequency drift of ZP likely result from time variations in properties of the fast electrons injected into the field well above the $\mathrm{ZP}$ source. The continuum source is comparatively more extended in size, with its emission centroid separated from that of the zebra source in the NE-SW direction. 
fast electrons injected into the field well above the ZP source. The continuum source is comparatively more extended in size, with its emission centroid separated from that of the zebra source in the NE-SW direction. The continuum emission requires less stringent conditions for the anisotropic distribution of the injected electrons and can therefore have a different size and a different source centroid location. This may also explain why the continuum emission is comparatively more stable in time and frequency. 


\section{Chapter 3}

\section{Karl G. Jansky Very Large Array "Solar Mode" Commissioning*}

${ }^{*}$ This chapter will soon appear as a memoranda for the $V L A$. This commissioning work was part of the NRAO RSRO program VLA/11B-129, for which I served as the primary resident observer in coordination with the local NRAO staff to perform hardware tests and develop solar observing and calibration strategies. This work was supervised by Tim Bastian, and received major help from Rick Perley, Michael Rupen, Ken Sowinski, Bryan Butler and many other NRAO staff. 


\subsection{Introduction}

The $V L A$ is a sensitive general-purpose radio interferometer that performs Fourier synthesis observations of celestial objects from 1-50 GHz. Unlike the FST discussed in the previous chapter, the $V L A$ is a true imaging instrument because it measures a large number of antenna baselines and hence, Fourier components, instantaneously. The Sun is a unusually intense and variable radio source. To enable solar observation with the $V L A$, special provisions must be made. This chapter is about the $V L A$ "solar mode" commissioning, which includes discussions of the hardware needed to enable solar observing, the tests conducted to characterize the hardware, and the strategies developed to observe the Sun and to calibrate the observations.

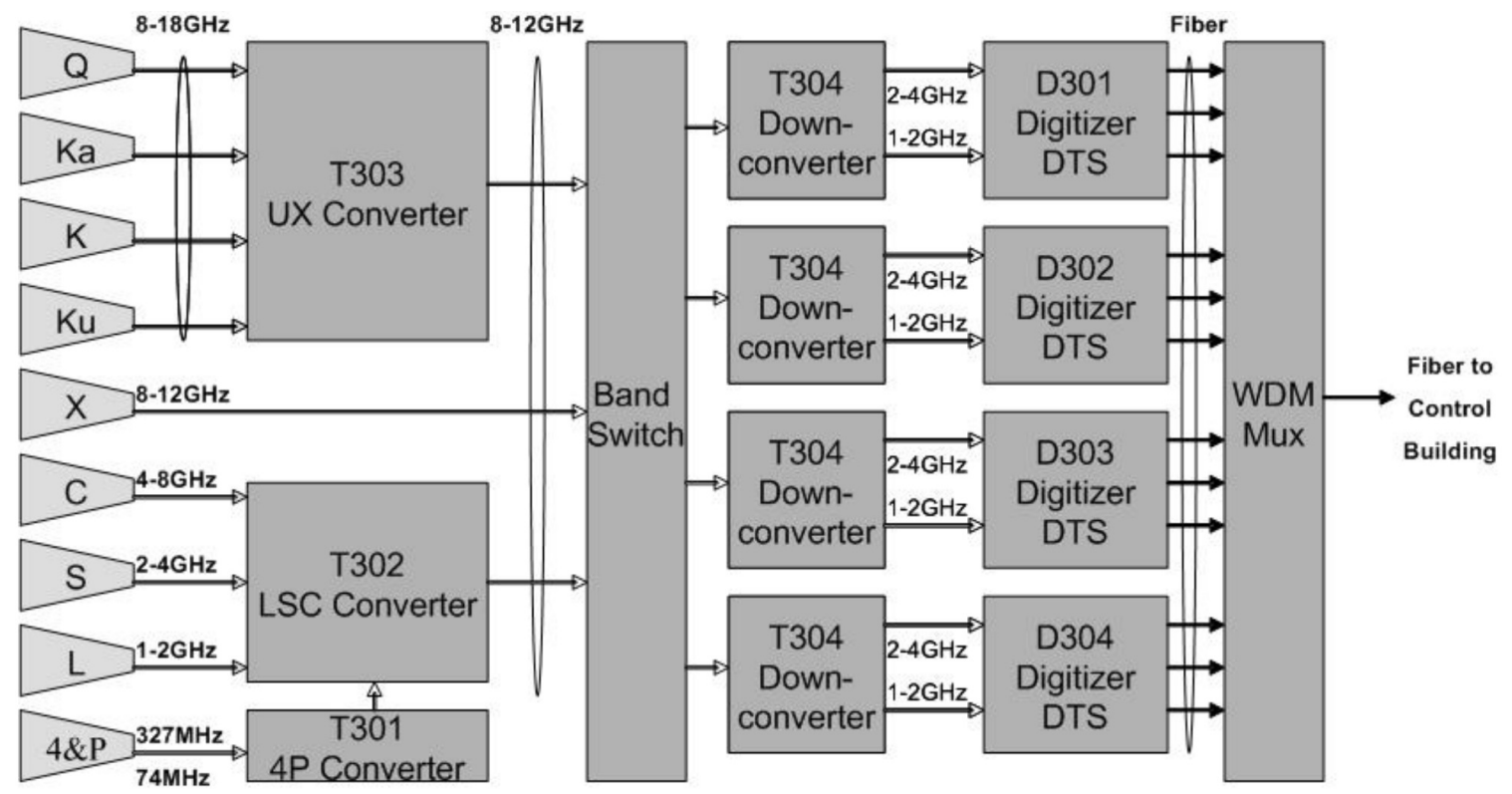

Fig. 3.1.- Overview of the $V L A$ 's electronics system, following the signal path from its entry into a particular receiver to the correlator (from McKinnon \& Perley, 2001).

Figure 3.1 provides an overview of the $V L A$ 's receiver and intermediate frequency (IF) system, following the signal path from its entry into a particular feed to the 
correlator. The $V L A$ is now supporting continuous frequency coverage from 1 to 50 $\mathrm{GHz}$ in eight different bands: L (1-2 GHz), S (2-4 GHz), C (4-8 GHz), X (8-12 $\mathrm{GHz}), \mathrm{Ku}(12-18 \mathrm{GHz}), \mathrm{K}(18.0-26.5 \mathrm{GHz}), \mathrm{Ka}(26.5-40.0 \mathrm{GHz})$, and Q (40.0-50.0 $\mathrm{GHz}$ ). The received radio signal enters one of the eight feed horns (located at the antenna's secondary focus) as two circular polarizations and is directed into the cryogenic, low-noise receivers, in which the signal is amplified by the low-noise amplifiers (LNA). Then the signal undergoes frequency conversions because it is technically more convenient for the electronic system to work in an IF range. Depending on the frequency band used, the two polarized signal channels are then either upconverted (in the T302 LSC Converter for the L, S, and C bands) or downconverted (in the T303 UX converter for the $\mathrm{Ku}, \mathrm{K}, \mathrm{Ka}$, and Q bands) into the 8-12 GHz band as four IF channels. No frequency conversion is needed for the $\mathrm{X}$ band signal as it is already in this frequency range. Band switches select the four 8-12 GHz IF channels from a given receiver and direct them into the four T304 frequency downconverter modules, in which each IF channel is split into two signal paths and downconverted to two 2-4 GHz basebands. The T304 downconverter modules employ input and output digital stepped attenuators and gain slope equalizers to ensure that optimum signal levels are input to the 3-bit 4 Gsps digitizers following the T304 modules. Alternatively, it is possible to select one of the two $2-4 \mathrm{GHz}$ signals in each IF channel and further downconvert them to 1-2 GHz. The signal is leveled by the digital stepped attenuators and input into the 8-bit 2 Gsps digitizers. This latter path was used for the solar observations discussed there.

There, the digitized signals are requantized (see Section 3.2.1) and cross-correlated for all antennas pairs and polarization channels to produce raw complex visibilities. Once calibrated, they are effectively Fourier components of the radio brightness dis- 
tribution. They can be Fourier inverted using data post-processing techniques to produce images of the radio source as a function of frequency and time, as described in Chapter 4.

The $V L A$ is designed for observing faint cosmic radio sources in the "cold" sky. Under such circumstances, the system noise (characterized by the system temperature $\left.T_{\text {sys }}\right)$ is usually dominated by receiver noise. However, when observing the Sun, this is no longer the case $-T_{\text {sys }}$ is dominated by the signal from the source (characterized by the antenna temperature $\left.T_{\text {ant }}\right)$ at all $V L A$ frequency bands. The total power will increase by 100-1,000,000 times from the cold sky values, depending on the level of solar activity. Moreover, the Sun is a highly variable object due to the occurrence of flares and other active phenomena, the main topic of this dissertation. For example, during a large solar flare, the radio flux density can change by several orders of magnitude in a time scale of tens of seconds to minutes.

Table 3.1: The effects of solar observing with the $V L A$

\begin{tabular}{rccccccc}
\hline \hline Band & $\begin{array}{c}\text { Freq. } \\
\mathrm{GHz}\end{array}$ & $\begin{array}{c}S_{\nu}(\mathrm{QS}) \\
\mathrm{SFU}\end{array}$ & $\begin{array}{c}S_{\nu}(\mathrm{FL}) \\
\mathrm{SFU}\end{array}$ & $\begin{array}{c}T_{\text {ant }}(\mathrm{QS}) \\
\times 10^{3} \mathrm{~K}\end{array}$ & $\begin{array}{c}T_{\text {ant }}(\mathrm{FL}) \\
\times 10^{3} \mathrm{~K}\end{array}$ & $\begin{array}{c}T_{\mathrm{QS}} / T_{\text {cold }} \\
\mathrm{dB}\end{array}$ & $\begin{array}{c}T_{\mathrm{FL}} / T_{\text {cold }} \\
\mathrm{dB}\end{array}$ \\
\hline $\mathrm{L}$ & $1-2$ & 50 & 200 & 40 & 126 & 33 & 38 \\
$\mathrm{~S}$ & $2-4$ & 130 & 200 & 40 & 159 & 32 & 38 \\
$\mathrm{C}$ & $4-8$ & 225 & 200 & 20 & 200 & 28 & 38 \\
$\mathrm{X}$ & $8-12$ & 420 & 200 & 15 & 238 & 26 & 38 \\
$\mathrm{Ku}$ & $12-18$ & 500 & 200 & 7.5 & 188 & 23 & 37 \\
\hline \hline
\end{tabular}

Table 3.1 summarizes the expected effect on the $V L A$ receivers from observing the Sun (adapted from Perley \& Bastian, 2004). The first two columns are the band names and frequency range corresponding to each. The third and fourth columns are the flux density in solar flux units $\left(1 \mathrm{sfu}=10^{4} \mathrm{Jy}=10^{-22} \mathrm{~W} \mathrm{~m}^{-2} \mathrm{~Hz}^{-1}\right)$ for the entire quiet Sun and for a medium-sized flare, with the expected antenna temperature given 
in columns 5 and 6 . The antenna temperature is evaluated via

$$
T_{A}=\frac{A_{e}}{\lambda^{2}} \iint T_{b}(\theta, \phi) P_{n}(\theta, \phi) d \Omega
$$

where $A_{e}$ is the effective antenna collecting area, $\lambda$ is the wavelength, $T_{b}(\theta, \phi)$ is the brightness temperature distribution of the Sun, and $P_{n}(\theta, \phi)$ is the normalized antenna gain. For quiet Sun observing at the low frequency bands (L and S), where the antenna primary beam is greater than the angular size of the Sun, the integral is obtained using the known power pattern of the $V L A$ antennas. At high frequency bands where the primary beam is smaller than the Sun, a beam efficiency of $75 \%$ is assumed. For flares, the source size is typically small compared to the primary beam, and the antenna temperature is approximated by $T_{A} \approx A_{e} \epsilon S /\left(2 k_{B}\right)$, where $\epsilon$ is the antenna efficiency. Columns 7 and 8 show the ratio of the expected system temperature while observing the quiet Sun and a medium-sized flare to that for cold sky in decibel values ( $\mathrm{dB}$, a logarithmic unit describing the ratio $R$ of a quantity relative to a given reference level $L_{\mathrm{dB}}=10 \log _{10} R$ ). For a typical flare, the antenna temperature may increase by a factor of a few to $\lesssim 100$ over the quiet Sun values. For extreme flare events, an increase in antenna temperature by a factor of up to $10^{6}$ over the quite Sun values is possible. Such a combination of high input power and rapid time variation places a great challenge for the $V L A$ to do solar observations, which will be detailed in the next section.

Another challenge is solar data calibration. After the radio measurements are made, they must be further processed to remove the effects due to the environment and the instrument itself. Calibration of interferometric radio data usually involves utilizing observations of standard point-source calibrators with known flux density, positions, and spectral properties to solve the antenna-based complex gains (am- 
plitudes and phases) and then apply these solutions to the raw visibility data (see Taylor et al., 1999, for details). However, the standard cosmic calibrators only have flux densities of at most several tens of Janskys, which is $\gtrsim 10^{5}$ times lower than the value of the quiet Sun. The telescope should be able to accommodate such vastly different values of flux densities in order to effectively calibrate the solar data against the reference calibrator sources.

Both of these elements - characterizing the hardware needed for solar observations and calibration of solar mode data - were the major part of the VLA "solar mode" commissioning work, carried out from September 2011 to March 2012 as part of the Resident Shared Risk Observing (RSRO) program entitled "Dynamic Imaging Spectroscopy of Coherent Solar Radio Bursts" (VLA/11B-129). Here we only discuss solar observing in the $\mathrm{L}, \mathrm{S}$, and $\mathrm{C}$ bands $(1-8 \mathrm{GHz})$, because hardware necessary for solar observations has only been outfitted in these bands at the time of this dissertation. Ultimately, the $V L A$ will support solar observing from 1-18 GHz, covering the L, S, $\mathrm{C}, \mathrm{X}$, and $\mathrm{Ku}$ bands. The hardware implementation and tests will be discussed in Section 3.2. The corresponding observing and calibration strategies will be discussed in Section 3.3. Science results based on observations that exploit the new $V L A$ solar mode will be discussed in Chapter 4 .

\subsection{Hardware Implementation and Tests}

\subsubsection{System Linearity}

Generally, for a properly functioning system, the recorded data values should be linearly proportional to the input signal strength. Observations of the Sun can drive the $V L A$ 's electronics system to power levels beyond their designed range, which result 
in signal compressions - the recorded value is considerably lower than that expected based on a linear relationship - and prevent proper use of the observational data. As a result, we need to characterize the region of linearity in which the system can perform properly without suffering significant compressions. Several elements in the signal path can be the source of signal compressions. Those include amplifiers in the receivers and the frequency converters, as well as other elements in the IF system, as discussed next.

\section{Amplifier Compression}

The $V L A$ amplifiers can suffer signal compressions given an input power level above the designed values. Figure 3.2 shows the relationship between the input and output power levels for a typical $V L A$ amplifier in dBs relative to the nominal (cold sky) values (from Perley \& Bastian, 2004). In general, a $1 \mathrm{~dB}$ compression (25\%) marks the end of the amplifier range that can be properly used, which occurs at about 36 $\mathrm{dB}$ above the nominal value of the input power level. From the estimated values of system temperature increases listed in Table 3.1, the standard $V L A$ receiver system should have the dynamic range necessary for observations of the quiet Sun, active regions, and moderate flares, but will not be capable of observing flares with a radio flux exceeding a few $\times 100$ sfu. A special signal path has been designed for L band observations to accommodate large flares. It does so by effectively using high noise amplifiers rather than LNAs in the front end. This system, while prototyped, has not yet been deployed at this time. 


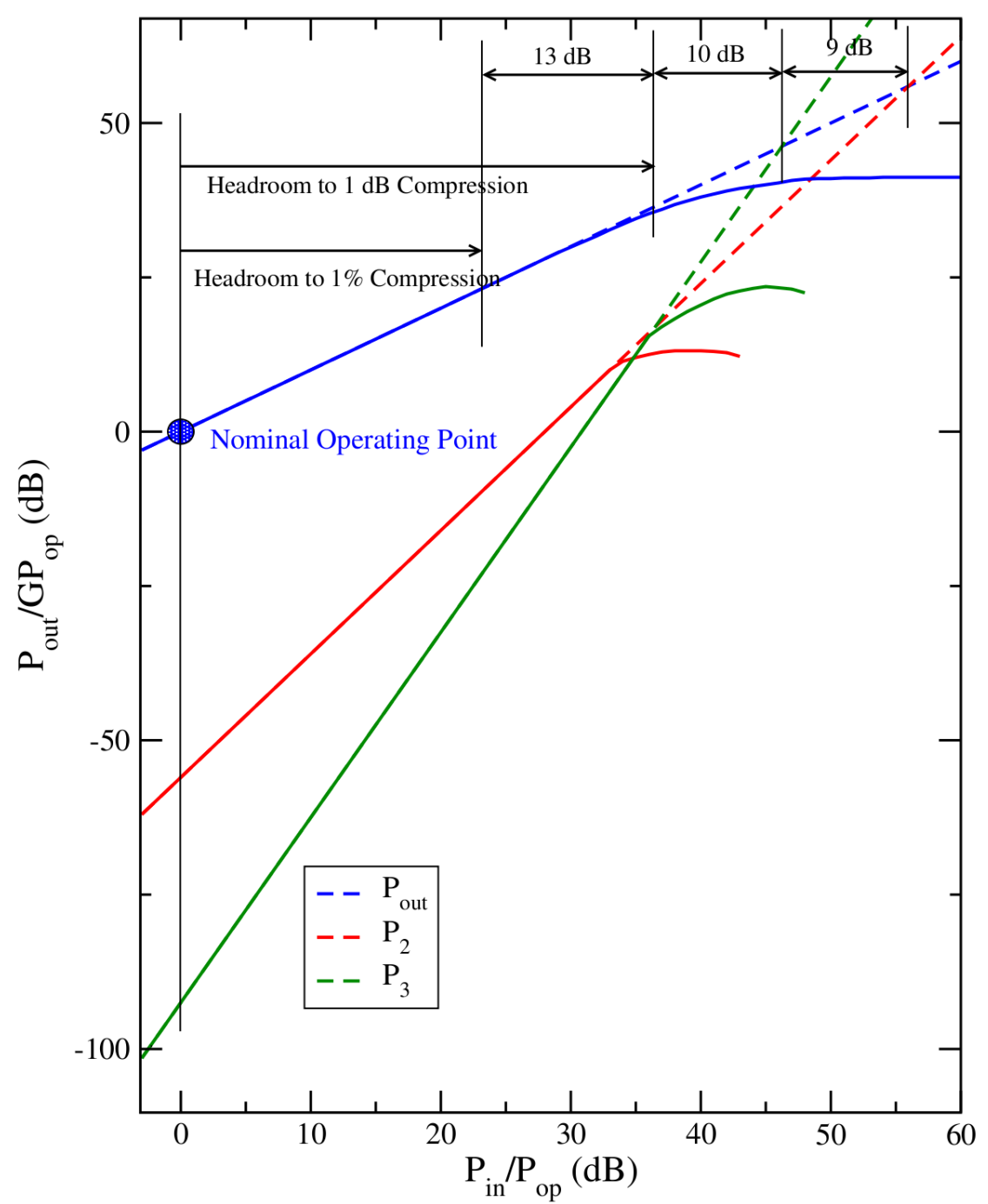

Fig. 3.2.- Compression from amplifiers of the $V L A$. The blue line shows the relationship between the input and output power for a typical $V L A$ emplifier. The displayed values in both axes are in $\mathrm{dBs}$ relative to the nominal (cold sky) power levels. The red and green lines are the powers in the second and third order intermodulation products. The compression is represented by the roll-off in output power as the input power exceeds a certain value (the "non-linearity" region). In general, an $1 \mathrm{~dB}$ compression (25\%) marks the end of the amplifier range that can be properly used, which occurs at about $36 \mathrm{~dB}$ above the nominal value of the input power level (from Perley \& Bastian, 2004). 


\section{Compression from the IF system}

Following the receiver LNAs, various other elements in the IF electronics system may cause compression, too. However, it is not practical to test every electronic device individually in the IF system for signal compression. Instead, on-sky tests utilizing the T304 step attenuators to manually change the internal power level can give us some insights. We did such a test as below: we observed a standard calibrator source and gradually varied the output power level from $-12 \mathrm{~dB}$ to $+14 \mathrm{~dB}$ relative to the nominal value $(-30 \mathrm{dBm} ; \mathrm{dBm}$ is the ratio in $\mathrm{dB}$ of the measured power referenced to one milliwatt) by stepping through the attenuations of the output attenuator in the T304 downconverters, and then measured the resulting antenna gain changes (in $\mathrm{dB})$. The results are shown in Figure 3.3 on a typical antenna, in which the $X$-axis is the output power level in $\mathrm{dBm}$ and the $Y$-axis is the antenna gain with an arbitrary normalization. The nominal output power level is marked by the vertical dotted line at $-30 \mathrm{dBm}$. It can be seen that the system behaves linearly from $\sim-42 \mathrm{dBm}$ to $\sim-25 \mathrm{dBm}$, until it experiences a $1 \mathrm{~dB}$ compression at $\sim 5 \mathrm{~dB}$ above the nominal output power level, which sets the safety zone in which the system can be operatedthe "headroom" - for a given observation. Such a compression exists on almost all antennas and varies subtly across different antennas. As the system power ahead of the T304 output attenuators was kept to be at an optimal level throughout the test, the IF compression is introduced downstream from the T304 output. It is suspected that the 8-bit digital samplers might be the cause of the compression. The fixed digital bits of sampling the input signal limit the dynamic range of samplers. In particular, a weak input signal is only sampled by a few bits, resulting in an excess quantization noise, while a strong input signal overrides all bits available, leading to excessive clipping in the bit edge and hence, compression. The estimated dynamic range of 
the $V L A$ 8-bit samplers is about $16 \mathrm{~dB}$ and the headroom above the nominal power level allowed by the samplers is $\sim 5 \mathrm{~dB}$, close to the test results. In addition, we note that the VLA's WIDAR correlator only correlates 4-bit digital samples for the time being. Hence the 8-bit digital samples from the antenna have to be "requantized" to 4-bit before entering the correlator. In order to maintain an optimum state of the requantizers, it is also desirable to reset their gains when the output of the 8-bit samplers various significantly, which likely occurs when switching between solar and calibrator scans, as well as during solar flares.

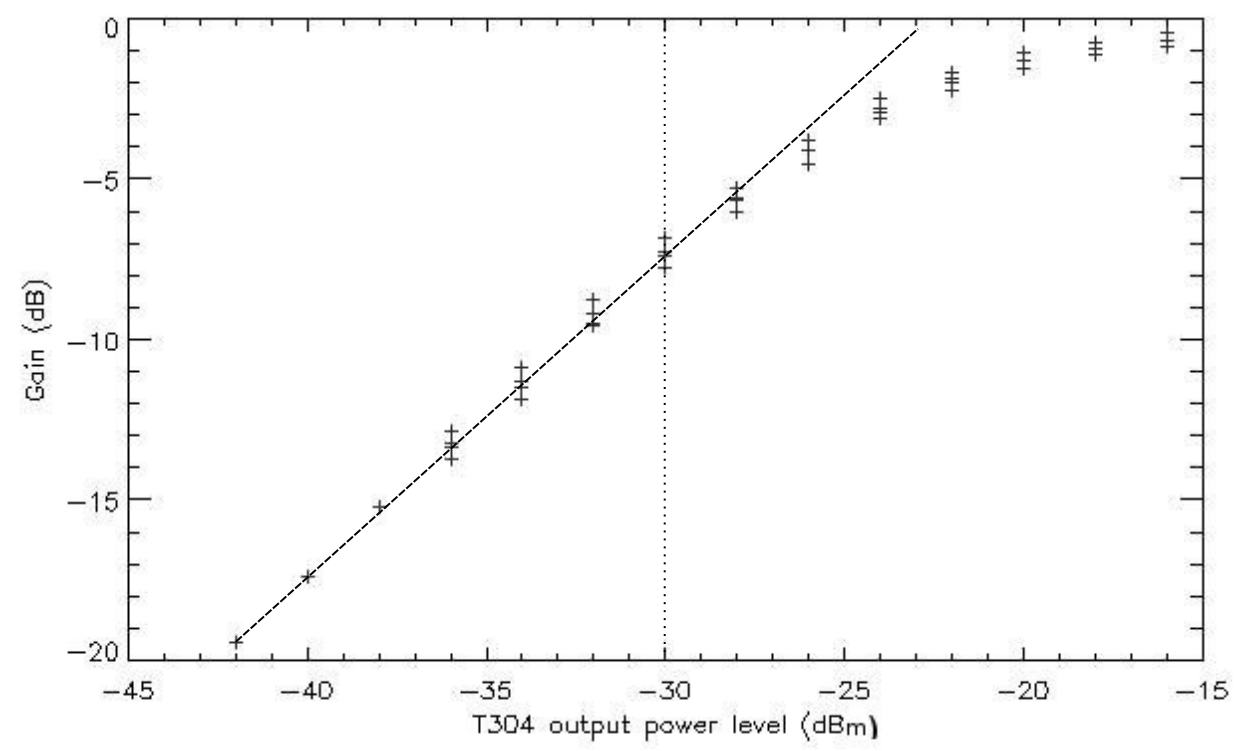

Fig. 3.3.- Compression from the IF electronics system of the VLA on a typical antenna. The plus symbols show the response of antenna gains at the four IF channels measured against the varying output power level with $2 \mathrm{~dB}$ steps from $-12 \mathrm{~dB}$ to $+14 \mathrm{~dB}$ relative to the nominal value, which is $-30 \mathrm{dBm}$ indicated by the vertical dotted line. The $1 \mathrm{~dB}$ compression (25\%) occurs at about $-25 \mathrm{dBm}, 5 \mathrm{~dB}$ above the nominal value of the output power level. 


\subsubsection{Gain Reduction}

\section{0 dB Solar Attenuators}

The enormous increase in system temperature which occurs when observing the Sun (c.f., columns 7 and 8 in Table 3.1) is a great challenge for the $V L A$ electronics system to handle properly. Section 3.2.1 has shown that the VLA amplifiers have sufficient dynamic range to handle the expected system temperature increase of solar observations up to moderate flares. However, the IF system do not allow too much headroom above the nominal operating level. For these reasons, $20 \mathrm{~dB}$ solar attenuators have been outfitted in the T302 LSC converters for the L, S, and C bands, located directly after the first amplifiers in the receivers (LNAs), in order to bring down the system power level in the IF system when observing the Sun. As these attenuators are not used when observing the calibrator sources, the net changes in complex gain (amplitude and phase) introduced by the solar attenuators must be measured a priori for the sake of solar data calibration. The process of measuring the complex gain of the $20 \mathrm{~dB}$ attenuators was as follows: a) Observe a strong calibrator source with the $20 \mathrm{~dB}$ attenuators switched in and out on all antennas; the output power level was optimized to be $-10 \mathrm{~dB}$ and $+10 \mathrm{~dB}$ relative to the nominal value when the 20 $\mathrm{dB}$ attenuators were in and out the signal path respectively. b) Solve the antenna based complex gains for the two cases separately. c) Difference the complex gains of the two cases and obtain the net gain change introduced by the solar attenuators. The solar attenuators on a considerable number of antennas introduce a non-zero frequency-dependent phase change. Figure 3.4 shows examples of three antennas, for both right-hand- and left-hand-circularly polarizations. It can be seen that the solarattenuator-introduced phase varies with frequency in a linear fashion in all spectral windows. That is because adding solar attenuators into the electrical signal path 
results in a net delay $\Delta \tau$, which manifests itself as a linear phase slope as a function of frequency:

$$
\Delta \phi(\nu)=2 \pi \nu \Delta \tau,
$$

where $\Delta \phi(\nu)$ is the phase change at a frequency $\nu$ due to the delay $\Delta \tau$ (at zero frequency the expected phase change $\Delta \phi$ is expected to be zero, if the delay is the only contribution). The delay can be simply derived through a linear fit of the phase slope across the observed frequencies. Corresponding results are given in Table 3.2 in nanoseconds. Zero values in the table means the fitting results are less than the uncertainty, which is typically less than 0.003 ns. Although on most antennas the attenuators introduce a small delay with a value of less than a few percent of one nanosecond, Equation (3.2) says that such a small delay can still result in a sizeable phase change at $\mathrm{GHz}$ frequencies (e.g., a 0.01 ns delay gives $\Delta \phi=18^{\circ}$ at 5 $\mathrm{GHz}$ ). Therefore corrections of such delays is desired for effective calibration. Figure 3.5 shows the residual phases as a function of frequency on the same antennas as in Figure 3.4 after delay corrections. Most antennas show zero phases across the entire frequency range, consistent with the expectation that the $20 \mathrm{~dB}$ attenuators only contribute to the signal with a delay (with only one exception - antenna 20 in $\mathrm{LCP}$ - showing a residual phase offset of $\sim 50^{\circ}$, the cause of which is unclear but may be related to its on/off switch). The resulting amplitude reductions were found, however, to be less than the expected value of $20 \mathrm{~dB}$. We conclude that it was most likely due to compression from the IF system and is not intrinsic to the attenuators. Bench measurements have confirmed that the true amplitude reductions from these attenuators are indeed $20 \mathrm{~dB}$. It is also worth noting that such measurements on $20 \mathrm{~dB}$ attenuators have been repeated several times and the same results have been obtained. 
Table 3.2: Net delays introduced by the $20 \mathrm{~dB}$ solar attenuators.

\begin{tabular}{|c|c|c|}
\hline Antennas & RCP Delay (ns) & LCP Delay (ns) \\
\hline 1 & 0 & 0 \\
\hline 2 & 0.006 & 0.003 \\
\hline 3 & 0.008 & -0.008 \\
\hline 4 & 0 & 0.067 \\
\hline 5 & -0.006 & -0.014 \\
\hline 6 & 0.006 & 0 \\
\hline 7 & -0.017 & -0.022 \\
\hline 8 & 0 & -0.008 \\
\hline 9 & 0.006 & 0.011 \\
\hline 10 & 0.011 & -0.006 \\
\hline 11 & 0.017 & 0.011 \\
\hline 12 & -0.003 & 0 \\
\hline 13 & 0 & 0 \\
\hline 14 & -0.017 & -0.014 \\
\hline 15 & 0.011 & 0 \\
\hline 16 & 0.106 & 0.1 \\
\hline 17 & 0 & -0.006 \\
\hline 18 & 0 & -0.006 \\
\hline 19 & - & - \\
\hline 20 & 0.094 & $0.069^{\dagger}$ \\
\hline 21 & 0.114 & 0.108 \\
\hline 22 & 0 & 0 \\
\hline 23 & 0.186 & 0.194 \\
\hline 24 & 0 & -0.017 \\
\hline 25 & - & - \\
\hline 26 & 0.011 & 0.161 \\
\hline 27 & 0.006 & -0.011 \\
\hline 28 & 0.006 & 0 \\
\hline
\end{tabular}

$\dagger$ Antenna 20, LCP has a residual phase offset of $\sim 50$ degrees.

\section{T304 Downconverter Attenuators}

Additional attenuators exist in the T304 downconverters, as discussed in Section 3.1. They can in principle provide additional dynamic range. These attenuators are digital 

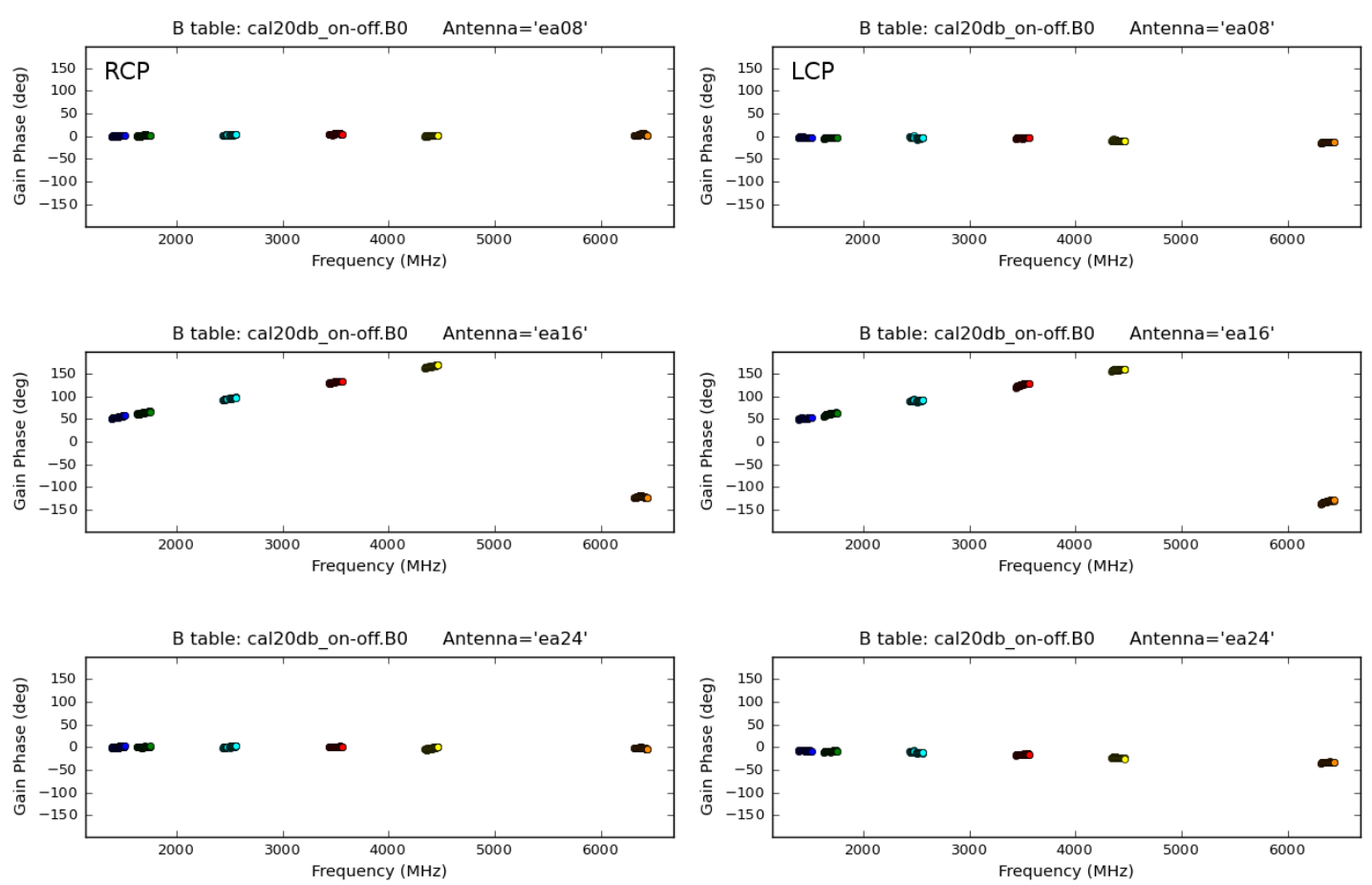

Fig. 3.4. - Examples of the net phase introduced by the $20 \mathrm{~dB}$ solar attenuators as a function of frequency. The phases were measured across the L, S, and C bands $\left(\sim 1.5^{-}\right.$ $6.5 \mathrm{GHz}$ ), sampled at six 256-MHz-wide subbands (points affected by RFI have been removed). It is evident that the phases vary linearly with frequency, corresponding to the delay result from the change of electrical path length.

step attenuators which can adjust their attenuation from 0-31 dB in $1 \mathrm{~dB}$ steps. Each attenuator has six major states of attenuation at $0.5,1,2,4,8$ and $16 \mathrm{~dB}$.

Similar to the $20 \mathrm{~dB}$ solar attenuators, the complex gain behavior should also be characterized for the purpose of effective solar observing and calibration, especially the phase variation. However, it turns out that such a characterization is very complicated and difficult to achieve in practice. The reason is that all the six major attenuation states on each attenuator have different phase behaviors as a function of frequency, as shown in Figure 3.6. The phase introduced by the T304 attenuators increases in frequency and in attenuation level. Measuring the frequency- and attenuationlevel-dependent phase properties of all the eight T304 attenuators on each of the 28 

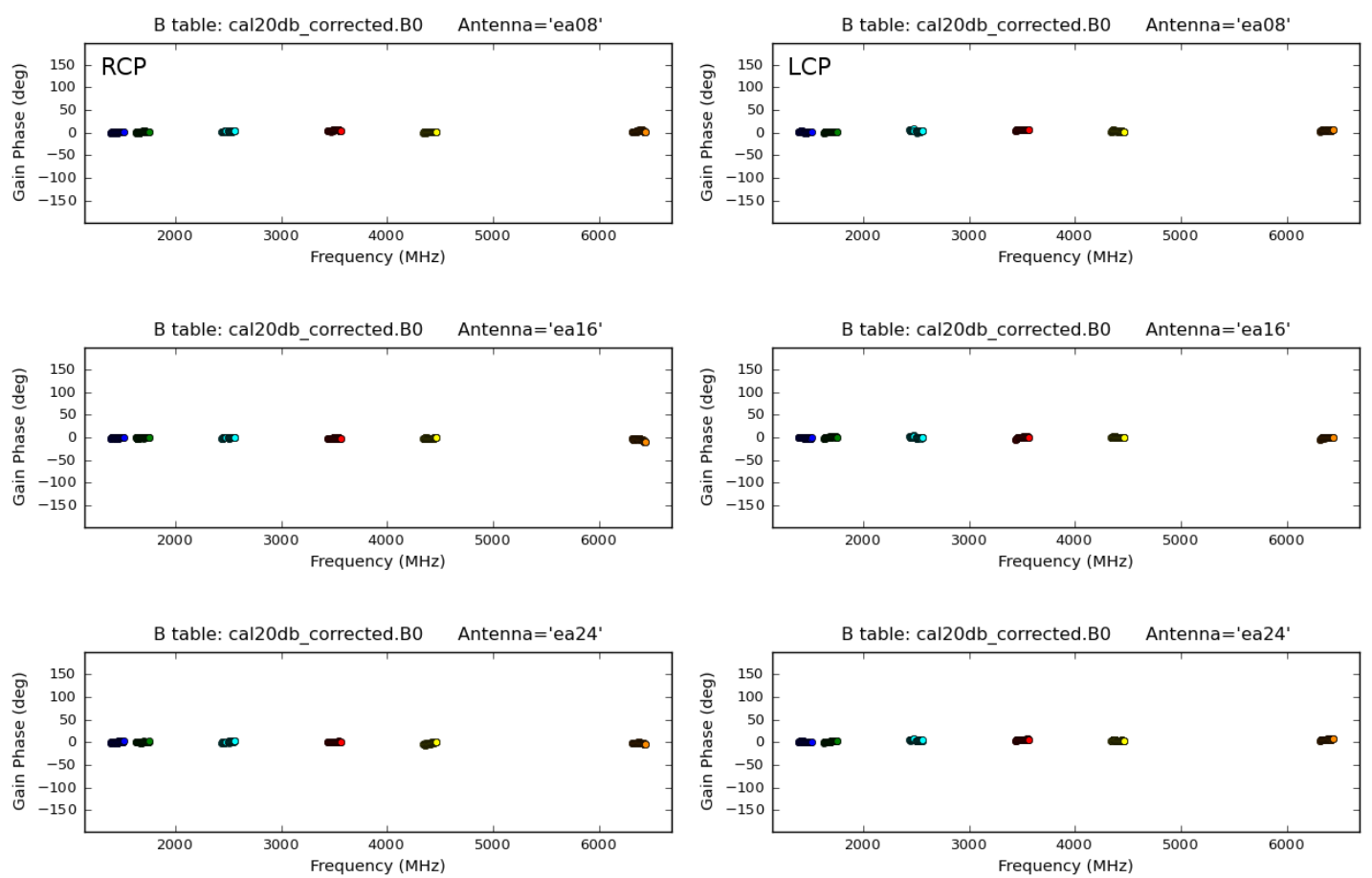

Fig. 3.5.- Residual phase as a function of frequency for the $20 \mathrm{~dB}$ solar attenuators after the delay correction.

antennas is impractical on the sky, but might be possible on the bench. However, the complexity of measuring the effects of all the T304 attenuators is avoided entirely by adopting an appropriate observing strategy, described in the next section.

\subsection{Solar Observing and Calibration Strategies}

The $V L A$ hardware properties relevant to solar observing can be briefly summarized as follows:

- The VLA amplifiers have a dynamic range sufficient for observing quiet Sun, active region, and moderate flares, but will suffer significant compression when observing larger flares. 


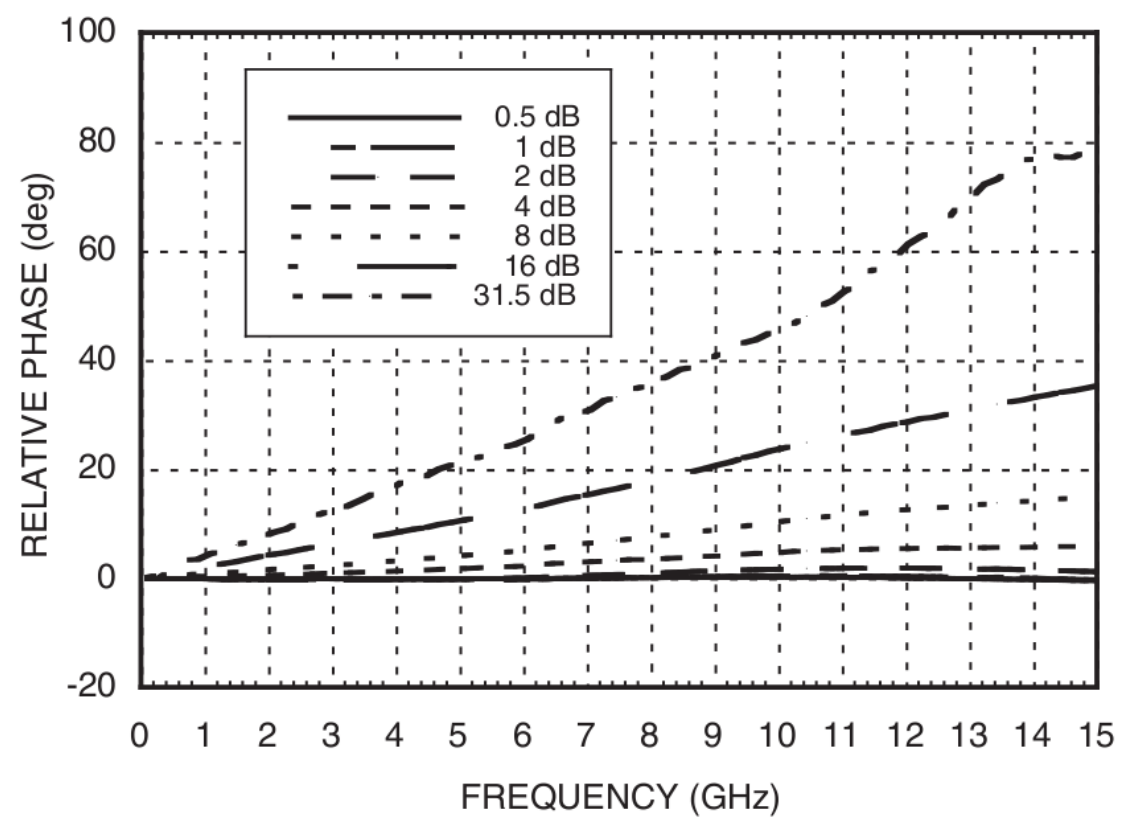

Fig. 3.6.- Phase as a function of frequency introduced by the T304 downconverter attenuators. Only those for major attenuation states are shown.

- The IF electronics system maintains its linearity from $\lesssim-42 \mathrm{dBm}$ to $\sim-25$ $\mathrm{dBm}$, providing a $\sim 5 \mathrm{~dB}$ headroom (a factor of 3 ) above the nominal output power level.

- The $20 \mathrm{~dB}$ solar attenuators have well-defined amplitude and phase properties. The net effect is to reduce the antenna gain by a factor of 100 and to introduce an antenna-based delay.

- The T304 downconverter attenuators allow additional dynamic range in the IF system, but their phase properties are complicated and difficult to characterize.

Based on these results, we discuss the corresponding strategies for solar observing and data calibration, as detailed below. 


\subsubsection{Solar Observing Strategies}

As the $V L A$ is designed to observe celestial radio sources, the system power levels are optimized for cold sky objects. For solar observing with effective calibration, we expect that the $V L A$ is able to observe both the Sun and cold-sky calibration sources. As shown in Table 3.1, solar observations can result in a $\gtrsim 30 \mathrm{~dB}$ increase in the system power level on the L, S, and C bands above the cold sky values, which exceeds the linear regime of the IF system. We use $20 \mathrm{~dB}$ solar attenuators to reduce the gain by a factor of 100 when observing the Sun. Any remaining gain reduction has to be absorbed by the T304 attenuators so that the power levels are again optimized to allow the system to work in the linear regime with an adequate headroom. In Section 3.2.2, we have shown that the differential complex gains introduced by the T304 attenuators are difficult to be calibrated. Therefore, we adopt a compromise solution: we optimize the T304 attenuator settings and save them while observing the Sun with the $20 \mathrm{~dB}$ attenuators in place. We then use the saved T304 attenuator settings when observing the calibrator sources. While power levels are no longer optimum when observing the calibrator sources $(\sim 10 \mathrm{~dB}$ below the nominal value for the L, S, and C bands), they nevertheless remain in the linear regime. This strategy enables us to solve for the complex gains of all antennas on the calibrator source and to transfer the solutions to the solar data, thereby calibrating the data without needing to solve for differential gains in the T304 module. The solar data then only need to be corrected for the complex gain introduced by the $20 \mathrm{~dB}$ solar attenuators. In addition, in order to let the digital requantizers work under their optimal states, we reset the gain of the digital requantizers at the beginning into the first of a series of calibrator scans and solar scans. For flare observations, the gain might need to be reset every a few minutes to accommodate the possible sudden variation in flux 
density during flares. This gain change should also be corrected. Details of data calibration will be discussed in the next section.

Under this scheme, a typical solar observing may contain the following elements:

- Observe the Sun with the $20 \mathrm{~dB}$ solar attenuator switched into the signal path, optimize the T304 downconverter settings, and save them.

- Observe a cosmic calibrator source with the $20 \mathrm{~dB}$ solar attenuator removed and leave everything else unchanged, i.e., the remembered T304 downconverter settings in the previous step are used.

- Reset the gain of the digital requantizers for both calibrator and solar scans.

- Repeat the Sun-calibrator observing cycle with the remembered T304 downconverter settings.

Such an observing scheme involving switching the $20 \mathrm{~dB}$ solar attenuators in and out of the signal path is, however, not yet integrated in NRAO's Observation Preparation Tool (OPT) system, which means the observing script can only be manually written for the time being. Future efforts will be made to make this process more automatic.

Finally, an effective solar observation with the $V L A$ requires careful planning, especially when one expects to observe transient events such as solar flares. Fortuitously, the $V L A$ now employs dynamic scheduling, which allows observations to be scheduled dynamically according to observing conditions as well as special needs for time-sensitive science. In our RSRO program, dynamic scheduling provided great flexibility for us to schedule our flare observations. By closely monitoring real-time solar activity and using a number of forecasting tools, a decision of scheduling can be made a few days in prior to the observation run, which increases the odds of catching solar flares. 


\subsubsection{Solar Data Calibration}

Interferometric data calibration is the process of determining various correction factors caused by the observing instrument and the environment that must be applied to the observed data in order to make them as close as possible to what an idealized interferometer would measure. The solar observing strategies suggested above require several extra steps in the data calibration process in addition to those in a "standard" calibration on a cosmic source dataset. First we briefly review the standard calibration procedure for a typical $V L A$ dataset on a cosmic source using the CASACommon Astronomy Software Applications - software package. A detailed discussion on data calibration with CASA can be found in Chapter 4 of the CASA Reference Manual \& Cookbook (http://casa.nrao.edu/docs/UserMan/UserMan.html), and we briefly reiterate below.

Figure 3.7 illustrates a standard calibration procedure with CASA (excluding the steps enclosed in the dashed boxes, which are for solar data calibration only). Calibration of a continuum dataset typically consists of the following steps:

1. Prior calibration: apply previously known calibration quantities, such as the calibrator flux densities and models using the task "setjy", antenna gain-elevation curves, delays, and antenna position offsets using the task "gencal".

2. Bandpass calibration: solve for the relative gain (amplitude and phase) across the observed frequency channels using the task "bandpass". A delay calibration using the "gaincal" task may be needed as the first stage to solve for the antennabased delays, which puts a phase ramp versus frequency channel in each spectral window.

3. Gain calibration: solve for the complex gain of each antenna and its variation 
as a function of time using the "gaincal" task.

4. Polarization calibration (if necessary): solve for polarization leakage terms and linear polarization position angle using the "polcal" task.

5. Flux density scaling: usually there is a primary calibrator with a well-measured flux density along with several secondary calibrators. The "fluxscale" task is used to rescale gain solutions and derive flux densities of the secondary calibrators.

6. Apply the calibrations: the task "applycal" is used to apply all the corrections derived in the previous steps to the visibility data.

The standard calibration procedure usually assumes that the radio signal follows the same path through the instrument electronics when observing the source and calibrators. Hence the complex gain of all antennas derived from calibrator scans can be directly applied to correct for the visibility data from the source scans. However, as outlined in Section 3.3.1, the solar observing strategies involve several steps which either use a different signal path or alter the system gain when observing the Sun. As a result, extra steps are required for solar data calibration, as illustrated in the dashed boxes in Figure 3.7.

First of all, $20 \mathrm{~dB}$ solar attenuators are only inserted into the signal path when observing the Sun. Therefore the net complex gain introduced by the $20 \mathrm{~dB}$ solar attenuators should be removed. Amplitude correction can be achieved by simply scaling up the solar visibility amplitude by a factor of 100. For phase correction, a multi-band delay correction should be performed. In CASA, this can be implemented with the "gencal" task using the "mbd" (multi-band delay) option.

Furthermore, in order to maintain an optimal state of the digital requantizers, 
we reset their gains for both calibrator and solar scans, which results in an addition change in gain as a function of time. This gain change also needs to be accounted for calibration. Currently, such records from the $V L A$ are only written in the "SY" table generated by the AIPS - Astronomical Image Processing System - software package as columns entitled "POSTGAIN". They can be applied to the data using the AIPS "TYAPL" task.

Finally, there is one particular advantage for solar observing with the $V L A$ - the solar data are almost immune to the radio frequency interference (RFI) effects caused by non-astronomical sources such as satellites, even on the low frequency bands where RFI is the most pronounced. This is because the system noise, which is dominated by the Sun itself, can be far greater than RFI signals. However, the calibrator scans used to derive the bandpass corrections for solar data are still strongly affected by RFI. As a result, no gain solution can be directly obtained from the RFI-dominated frequency channels for bandpass calibration. Fortuitously, for most spectral windows, only a small percentage of frequency channels is strongly affected by RFI. To make the most use of the data, for each spectral window, we use interpolated values on these frequency channels through polynomial fits based on all the RFI-free channels as approximate gain solutions to be applied to the solar data. This procedure can be realized in the CASA task "bandpass". Figures 3.8 and 3.9 show such examples of bandpass solutions in both amplitude and phase for a RFI-affected spectral window in the $\mathrm{L}$ band.

It should be noted that this treatment for bandpass calibration has to be evaluated on a case-by-base basis according to the severity of RFI effects, the quality of the interpolations, as well as the specific scientific requirements. Depending on practical needs, one can alternatively choose to directly interpolate the bandpass solutions of 
the RFI-affected channels from those of the nearby channels. A bandpass solution based on observations of the quiet Sun itself is also plausible, but detailed discussions are beyond the scope of this report. 
Input Data, Tables \& Information

Process

Output Data, Tables \& Information

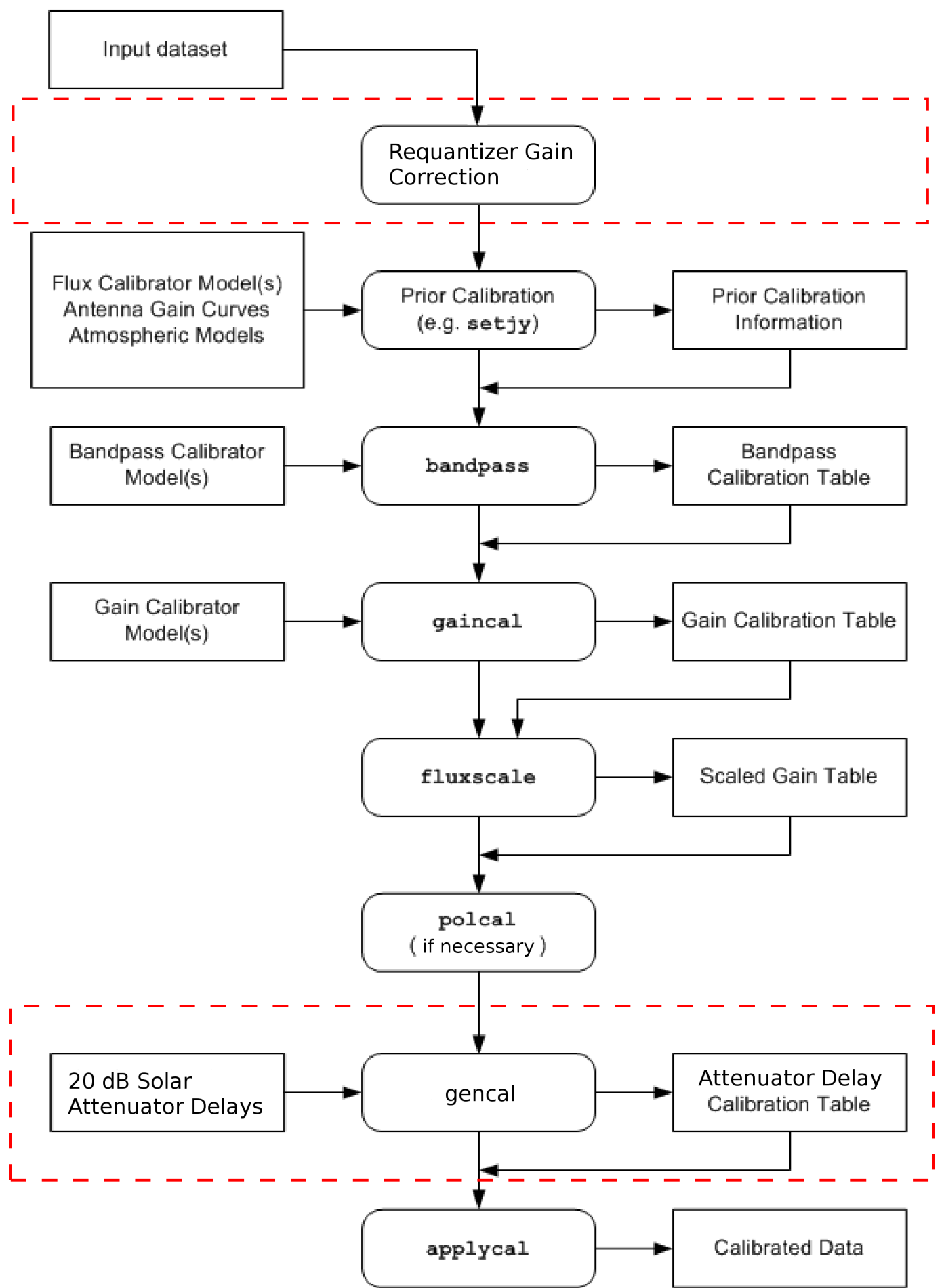

Fig. 3.7.- Work-flow diagram of a standard VLA data calibration procedure with CASA (adapted from Ott \& Kern, 2012). Steps enclosed in the dashed boxes are exclusive for solar data calibration. 

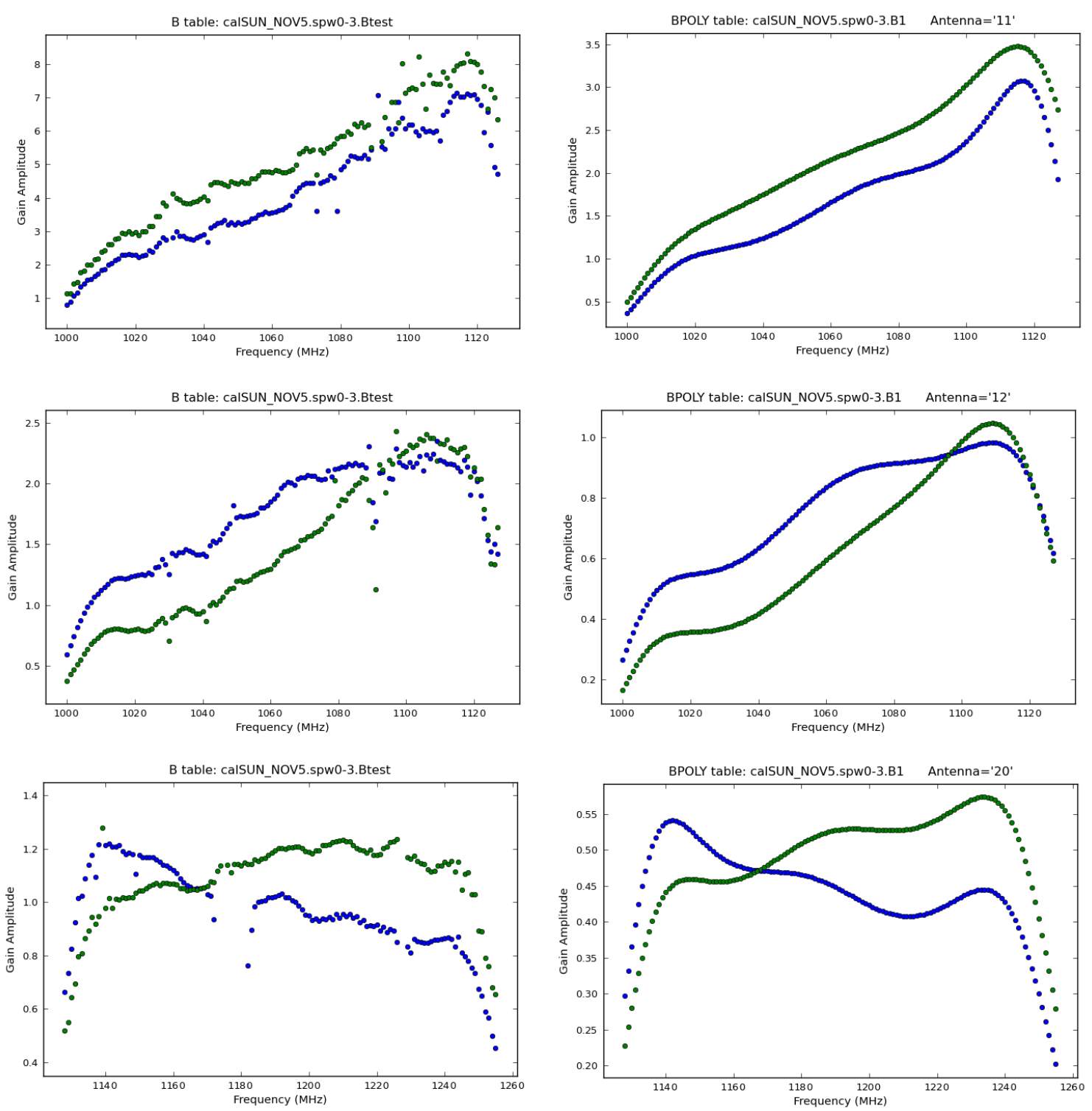

Fig. 3.8. - Examples of bandpass solutions of amplitude for one spectral window on a calibrator source using polynomial fits. Left panels are results obtained by a normal bandpass solution method. Solutions on several spectral channels are strongly affected by RFI and cannot be transferred to the solar data. Right panels are results of the same spectral window and antenna using polynomial fits on RFI-free spectral channels. These interpolated values are used as solutions for the RFI-affected spectral channels, hence they can be transferred to the solar data. 

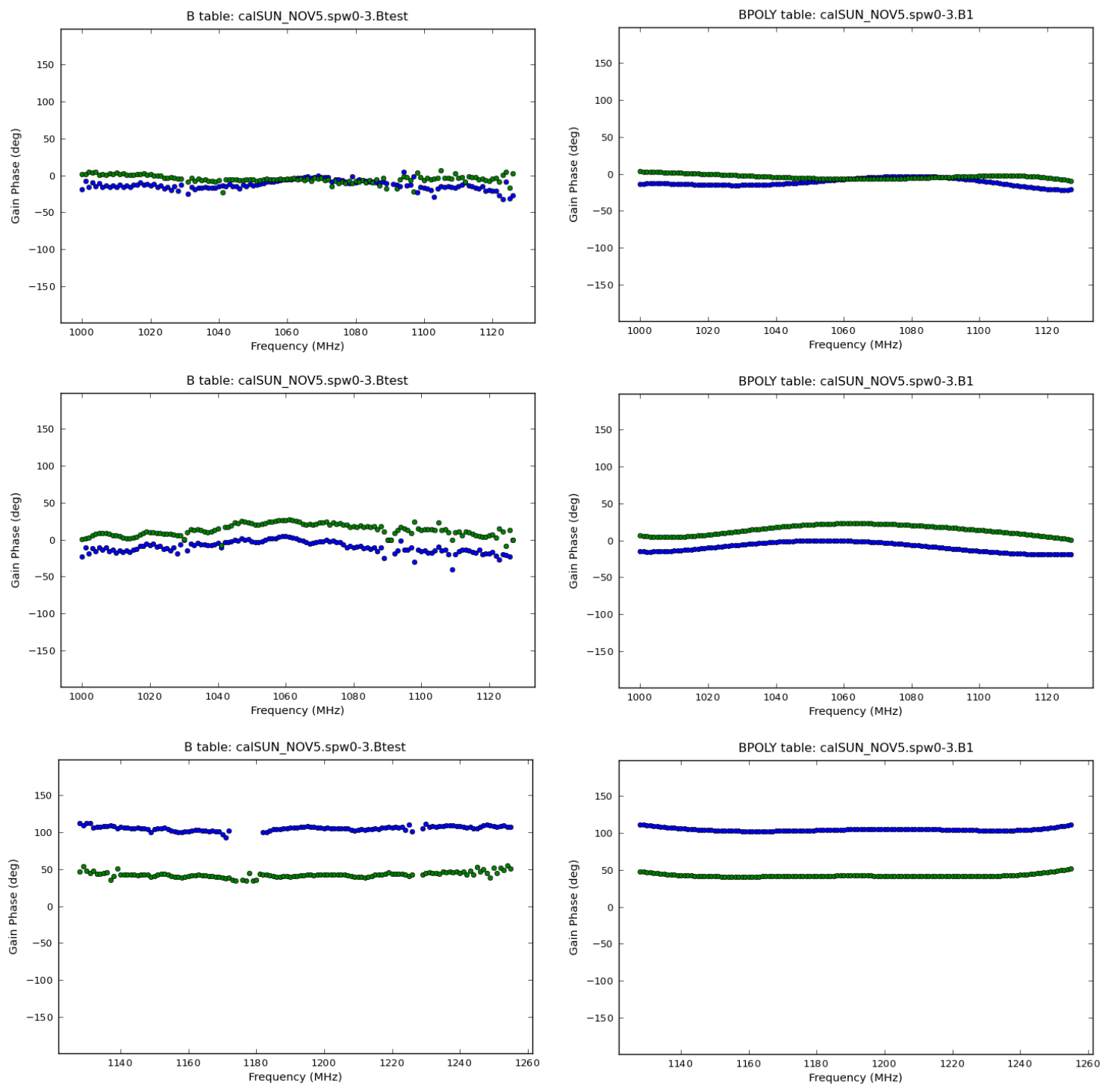

Fig. 3.9.- Similar to Figure 3.8, but instead showing bandpass solutions of phase for the same antennas and spectral window. 


\section{Chapter 4}

\section{Type III Radio Bursts: Traces of Propagating Electron Beams*}

*The major content is adapted from Chen et al. (2013). The publication resulted from the NRAO RSRO program VLA/11B-129, for which all the authors contributed to the observing proposal. The data reduction, scientific analysis and writing are primarily my work. Tim Bastian supervised the work, helped with data reduction, and contributed to writing and revision. Stephen White helped with the RHESSI HXR images. Dale Gary provided helpful comments and suggestions. 


\subsection{Introduction}

Certain types of solar activity — flares and jets - are powered by the impulsive release of energy through fast magnetic reconnection. In the standard scenario (see Chapter 1.2.2), a reconnection region located in low corona (Masuda et al., 1994; Sui \& Holman, 2003; Battaglia \& Benz, 2006) accelerates particles to non-thermal energies. These particles carry a large fraction of the released energy and play an important role in energy transport processes (Lin \& Hudson, 1976). Electron beams can form through the propagation process, if not from the acceleration mechanism itself (Bastian et al., 1998), and travel upward and downward in the corona along magnetic field lines. The downward-propagating beams will eventually be stopped by the dense chromospheric plasma and produce HXR emission. The chromospheric plasma is heated by these beams to high temperatures and expands hydrodynamically into the corona where it is observed in EUV and/or SXR wavelengths (Benz, 2008). However, direct information about these fast electron beams and the region from whence they originate has remained elusive.

One method to probe these beams is through radio observations. The radio signature of coronal electron beams, first discovered in the late-1940s at meter wavelengths (Payne-Scott et al., 1947; Payne-Scott, 1949; Wild \& McCready, 1950), is called a type III radio burst. It is the result of the nonlinear conversion of Langmuir waves generated by beam instabilities to electromagnetic radiation at the fundamental or harmonic of the local electron plasma frequency, $\nu_{\mathrm{pe}} \approx 8980 \sqrt{n_{e}} \mathrm{~Hz}$, where $n_{e}$ is the electron number density. Since $n_{e}$ varies with height in the corona, the radio emission from the electron beam drifts from high to low frequencies for upward-propagating beams, and in the opposite sense for downward-propagating beams. Their high fre-

quency counterpart - decimetric type III radio bursts (hereafter, type IIIdm bursts) - 
was first observed in the early 1960s (Young et al., 1961), and was subsequently found to be closely associated with impulsive energy release in flares (Kane, 1981; Dennis et al., 1984; Aschwanden et al., 1985; White et al., 2011). For these reasons, type IIIdm bursts are believed to be an important diagnostic of impulsive magnetic energy release.

A barrier to exploiting type IIIdm emissions, and of course, the other types of coherent radio bursts, has been the lack of true "radio dynamic imaging spectroscopy", where simultaneous imaging observations are available at each frequency and time that a burst is observed. Imaging observations of type III, type IIIdm, or type U bursts (a type III in a closed magnetic loop) have been reported at one, or a few, discrete frequencies (e.g., Aschwanden et al., 1992; Raulin et al., 1996; Aurass \& Klein, 1997; Paesold et al., 2001), but radio dynamic imaging spectroscopy has not been possible. Here we report the first use of radio dynamic imaging spectroscopy with the upgraded $V L A$ to observe type IIIdm bursts and other types of solar coherent radio bursts. In Section 4.2 we introduce the new technique of radio dynamic imaging spectroscopy enabled by the $V L A$. We also discuss the observational setup of the $V L A$ and show the first data. Section 4.3 presents observations of the type IIIdm bursts in the 2011 November 5 event and the associated multi-wavelength observations from a number of complementary instruments. In Section 4.4 we discuss the implications of these data and place them in an interpretive context. We conclude in Section 4.5. 


\subsection{From Dynamic Spectroscopy and Imaging to Dynamic Imaging Spectroscopy}

As mentioned in Chapter 1.2.3, in the past, exploration of solar coherent radio bursts has been largely proceeded using either dynamic spectroscopy or imaging observations, and in some cases, combination of the two from different instruments. Dynamic spectral studies of these bursts have played an important role in defining this research area, e.g., the classification of solar radio burst types I, II, III, IV, and V was largely based on the their dynamic spectral properties. Detailed spectral and temporal structures of these bursts have been used to provide rich information for identifying their emission processes and diagnosing the source plasma parameters. Alternatively, imaging of these bursts have contributed to revealing their source locations and establishing their connections to the flare energy release. To make further progress in this area, simultaneous dynamic spectroscopy and imaging is desired. In Chapter 2, I have demonstrated the use of spatially resolved dynamic spectroscopic observations obtained from a three-element array (the FST) in examining the burst emission mechanism, diagnosing the plasma parameters of the burst source, locating the burst source, and establishing its connection with the flare energy release. It is obvious that true dynamic imaging spectroscopic observations would provide a much greater potential in such studies and may, perhaps, reshape our understanding of solar coherent radio bursts.

As solar coherent radio bursts can sometimes occupy a wide range of radio frequencies and usually show very fine spectro-temporal structures (e.g., in dm- $\lambda$ range, fine structures can often be distinguished down to milliseconds in time and $\mathrm{MHz}$ in frequency), ideally, an instrument is desired to observe these bursts with simultane- 
ous imaging and dynamic spectroscopy over a large bandwidth at high spatial, time, and spectral resolutions commensurate with the properties intrinsic to these bursts. Such an instrument has not been available until very recently, after the $V L A$ has been upgraded with state-of-art receivers and electronics (Perley et al., 2011). While the $V L A$ is a general-purpose radio telescope, provisions were made to enable solar observing. In Chapter 3, we have presented the VLA "solar mode" commissioning work of characterizing the relevant hardware properties and deciding the strategies appropriate for solar observing and data calibration. After the commissioning, the $V L A$ now serves as the most advanced radio telescope currently available for solar observing.

The first solar observation of the upgraded VLA was made on 2011 November 5 from 20:29 to 23:08 UT when the VLA was in the D configuration, for which the longest antenna baseline is $1 \mathrm{~km}$. We observed in the $1-2 \mathrm{GHz}$ frequency band $(\lambda=15-30 \mathrm{~cm})$ using 1024 spectral channels, each $1 \mathrm{MHz}$ in bandwidth, with a time resolution of $100 \mathrm{~ms}$. Due to limitations in data throughput at that time, a maximum of 17 antennas could be used. These were nevertheless sufficient for imaging the source of radio emission. The angular resolution of the 17 -antenna array was $35^{\prime \prime} \times 95^{\prime \prime}$ at 1.5 $\mathrm{GHz}$ at the time of observation, which scales linearly with wavelength. Both senses of circular polarization were observed. Unfortunately, a hardware error resulted in the loss of most of the data from 1.5 to $2.0 \mathrm{GHz}$, rendering it unsuitable for imaging, although dynamic spectroscopic information was available. We therefore focus on the data from 1.0 to $1.5 \mathrm{GHz}$. Calibration of the antenna gains and frequency bandpasses was performed by referencing the observations to standard sidereal sources. When observing the Sun, $20 \mathrm{~dB}$ attenuators were inserted in the signal path. Their presence introduces fixed perturbations to the antenna gains that were measured prior to the 
observation and subsequently corrected by calibration (see Chapter 3.3). A number of type IIIdm bursts were detected during the observation. Detailed discussion of these bursts will be presented in the following sections.
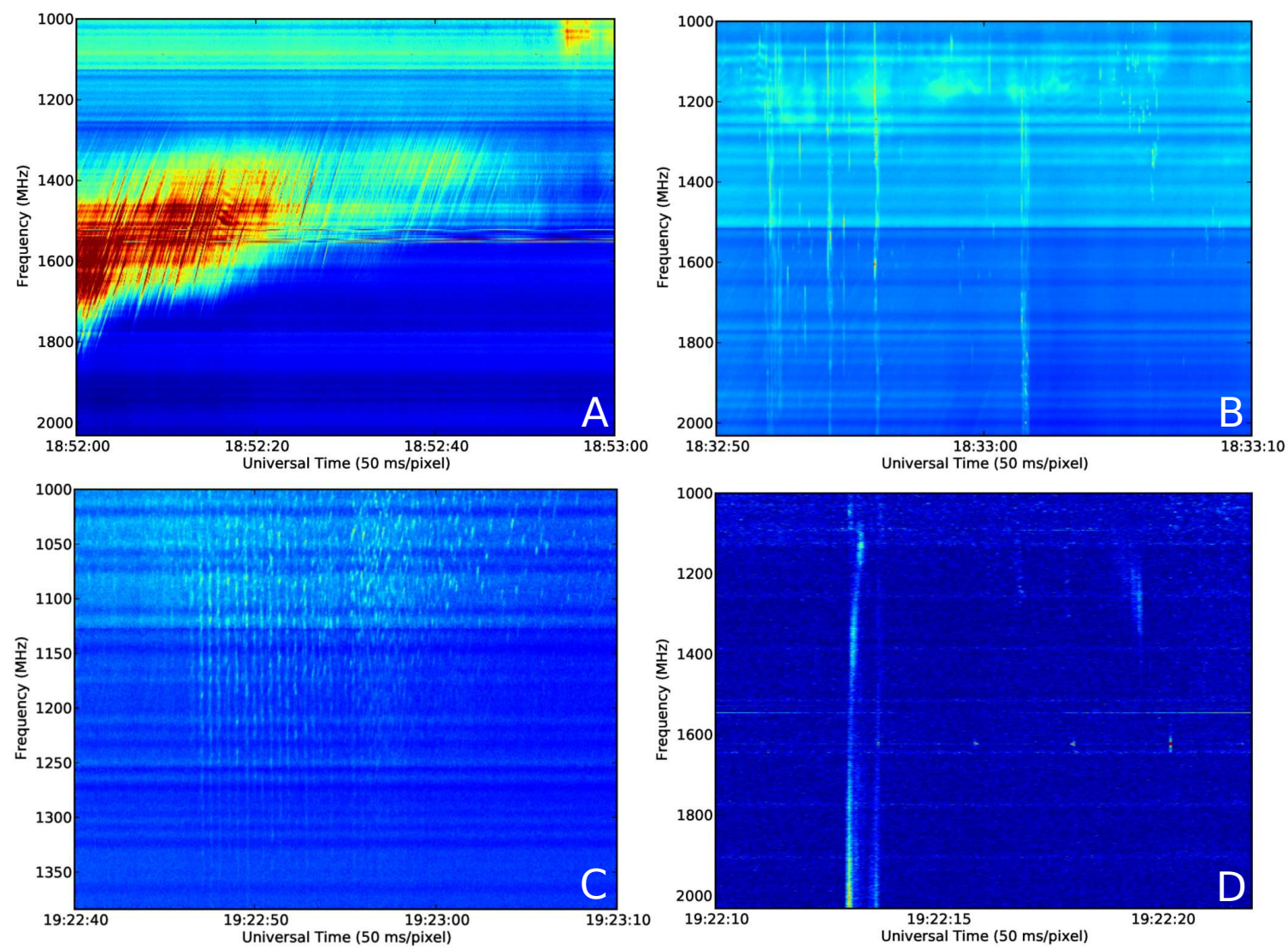

Fig. 4.1. - Examples of various types of solar coherent radio bursts observed by the VLA on 2012 March 3, including fiber bursts (A), spike bursts organized in broadband "envelopes" (B), zebra-pattern bursts (B), periodic reverse-drifting bursts with substructures (C), and type IIIdm bursts (D).

On 2012 March 3 from 17:39 to 21:45 UT, we performed another solar observation with the $V L A$ using the same spectral settings but with an improved time resolution of $50 \mathrm{~ms}$, doubling that of the 2011 November 5 observation. This time, the full 1-2 $\mathrm{GHz}$ frequency band functioned properly and was available for imaging. In addition, the array was in the $\mathrm{C}$ configuration, for which the longest antenna baseline is 3.4 
$\mathrm{km}$, making the spatial resolution three times better. The payback is that fewer (15) antennas were allowed because of an increasing rate of data throughput due to a better time resolution. The same observing and calibration strategies were used as those in the 2011 November 5 observation. We recorded a rich variety of coherent radio bursts in this event, a majority of which was associated with a long-duration GOES class C1.9 SXR flare. Figure 4.1 shows some examples of these bursts in the cross-power dynamic spectra, including fiber bursts (A), spike bursts organized in broadband "envelopes" (B), zebra-pattern bursts (B), periodic reverse-drift bursts with substructures (C), and type IIIdm bursts (D). Analysis of these data are currently ongoing and will not be discussed in this dissertation.

\subsection{Observations}

Using the $V L A$, on 2011 November 5, type IIIdm bursts were observed in association with a coronal jet during the aftermath of a GOES class M1.8 SXR flare (Figure 4.2(B)) in active region (AR) 11339. The cross-power dynamic spectra are shown in Figures $4.2(\mathrm{G})-(\mathrm{H})$, where each bright vertical or nearly vertical feature represents an individual burst. The largest group of bursts occurred between 21:21:40 and 21:22:10 UT (Figure 4.2(H)), well correlated with a peak in the $12-25 \mathrm{keV}$ HXR emission observed by the RHESSI satellite (Figure 4.2(I), the red curve), indicating

the release of non-thermal electrons. At 21:21:00 UT, another (weaker) HXR peak can be seen, during which the radio continuum has a small enhancement. A small cluster of narrow-band type III-like bursts can be found around the time of this HXR peak (Figure $4.2(\mathrm{G})$ ). The coronal jet was observed by the SDO/AIA (Figure 4.2(A)) during this energy release event, followed by subsequent brightening of closed loops (Figures 4.2(C)-(F)). The jet was also observed by the EUV Imager (EUVI) 
aboard STEREO B (Wuelser et al., 2004) that observed the Sun from the east (Figure 4.3). Interestingly, no concurrent interplanetary type III bursts were detected by the STEREO/WAVES (Bougeret et al., 2008).
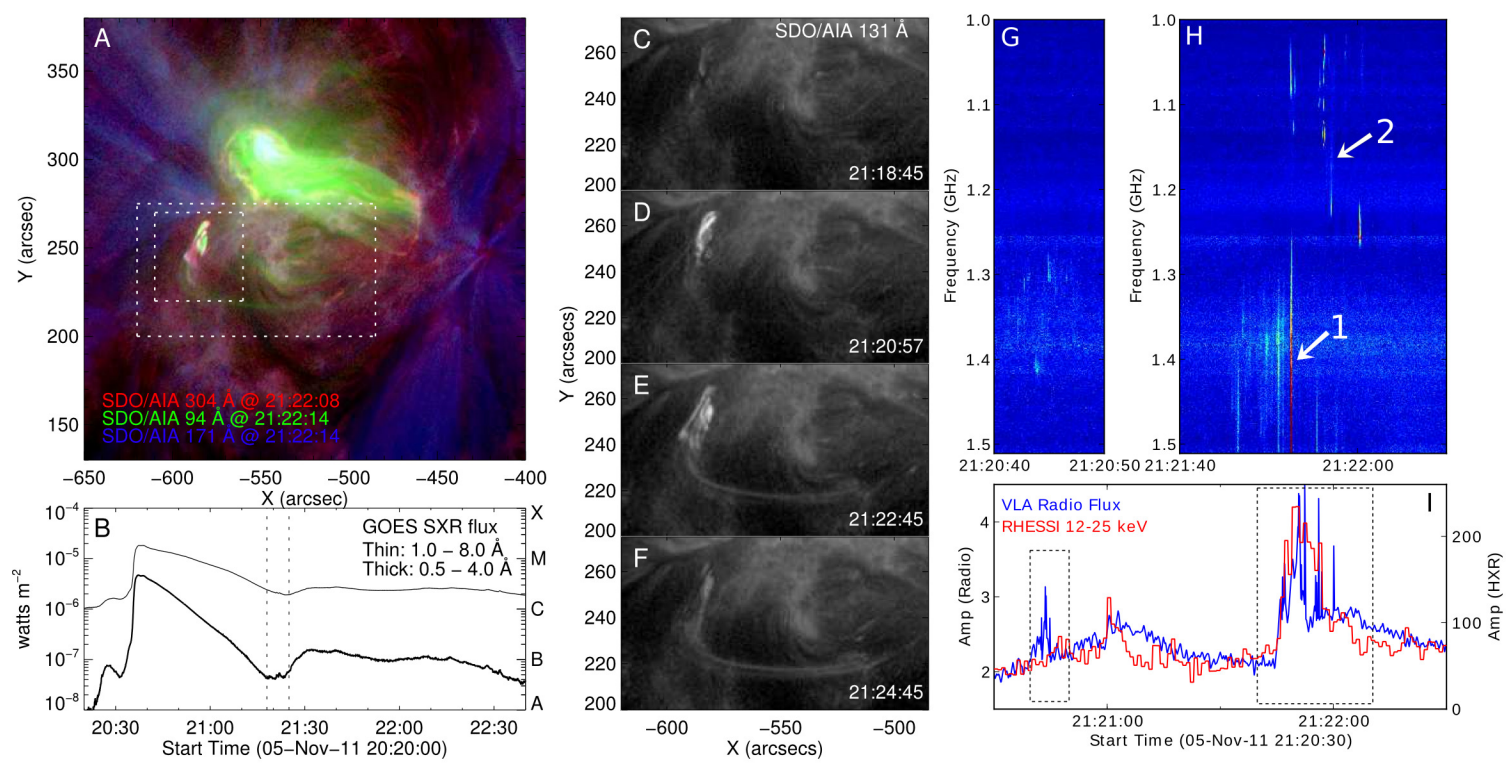

Fig. 4.2.- (A) Composite image of AR 11339 showing emission in $304 \AA$ (red, 0.1 MK), $94 \AA$ (green, $6 \mathrm{MK}$ ), and $171 \AA$ (blue, $0.8 \mathrm{MK}$ ) from SDO/AIA. (B) GOES SXR flux showing the M1.8 flare (peaks around 20:35 UT) and its aftermath. The coronal jet occurred between 21:18 and 21:25 UT (dashed vertical lines). (C-F) Dynamic evolution of the coronal jet and the subsequent loop brightening in AIA $131 \AA(0.4$ $\mathrm{MK}$ and $10 \mathrm{MK}$ ) images. The field of view (FOV) is indicated by the large box in Figure 4.2(A). (G-H) VLA dynamic spectra in which type IIIdm bursts (the bright vertical or nearly vertical features) are present. (I) $V L A$ radio (blue) and RHESSI 12-25 keV HXR (red) light curves from 21:20:30 to 21:22:30 UT, showing the temporal correlation of the radio flux and type IIIdm bursts with the HXR flux. The small and large boxes correspond to the time ranges of the dynamic spectra shown in $(\mathrm{G})$ and $(\mathrm{H})$.

Dynamic imaging spectroscopy with the $V L A$ allows each pixel in the dynamic spectrum to be imaged. Although the type IIIdm bursts themselves were unresolved by the $V L A$, the radio source centroid position can be determined with high accuracy. The high signal-to-noise ratio of these bursts allows their positions to be fit to 

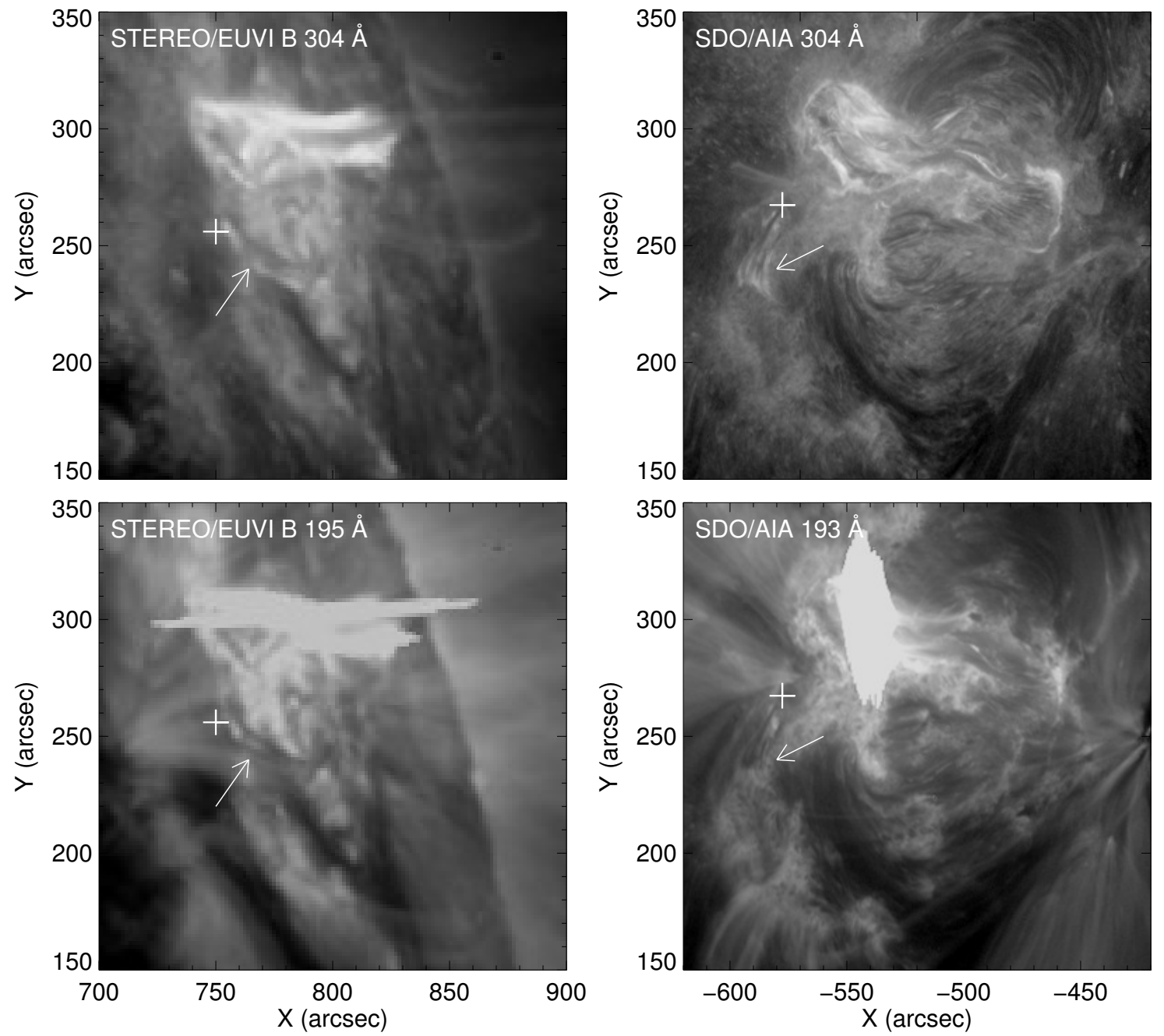

Fig. 4.3.- Coronal jet (marked by the white arrows) seen by both STEREO/EUVI B (left column) and $S D O /$ AIA (right column) at around 21:26 UT. SDO was viewing AR 11339 from the Earth, while the STEREO B satellite was trailing the Earth by $103^{\circ}$ at the time of the observation thereby viewing AR 11339 from the east. $X$ and $Y$-axes are aligned with solar east-west and south-north, respectively, in each perspective. White crosses mark the same location at the foot of the EUV jet, based on direct coordinate transformations. 
$\sim 1 \%$ of the nominal resolution, or $\lesssim 1 \mathrm{Mm}$. Examples of the fitted centroid locations for two temporally-resolved bursts (indicated by the arrows "1" and "2" in Figure 4.2H) are shown in Fig. 4.4(B)-(C) and Figure 4.4(D)-(G), colored from blue to red corresponding to increasing frequency. At any given time, the centroid locations at different frequencies follow well-defined trajectories, distributed from southwest (bottom right) to northeast (upper left) with increasing frequencies. All the trajectories fall within an envelope with the high-frequency end (high density, thus low coronal heights) originating near the location of the EUV jet and the HXR sources low in the atmosphere (Figure 4.4A, showing a superposition of all the type-IIIdm centroid locations from 21:20:30 to 21:22:10 UT). In addition, for bursts that are temporally resolved, the emission drifts from high to low frequencies with a rate of $0.3-1 \mathrm{GHz}$ $\mathrm{s}^{-1}$, and their centroid locations move to the southwest direction with time, indicating that the electron beams were propagating upward away from the EUV and HXR source (e.g., Figures 4.4(D)-(G)). These observations show the electron beam trajectories directly and, hence, the coronal magnetic field lines along which they streamed away from the reconnection site.

\subsection{Results and Discussion}

The close spatiotemporal association of the type-IIIdm-burst trajectories and the HXR footpoints (Figures 4.4(B)-(G)) suggests that the X-ray-producing downwardpropagating electron beams and the type-IIIdm-emitting upward-propagating electron beams originate from a common energy release site, confirming previous ideas based largely on the temporal correlation between HXR/type-IIIdm emission (Dennis et al., 1984; Aschwanden et al., 1995a). It also places the reconnection site at a location above the HXR footpoints and below the height of the highest-frequency 


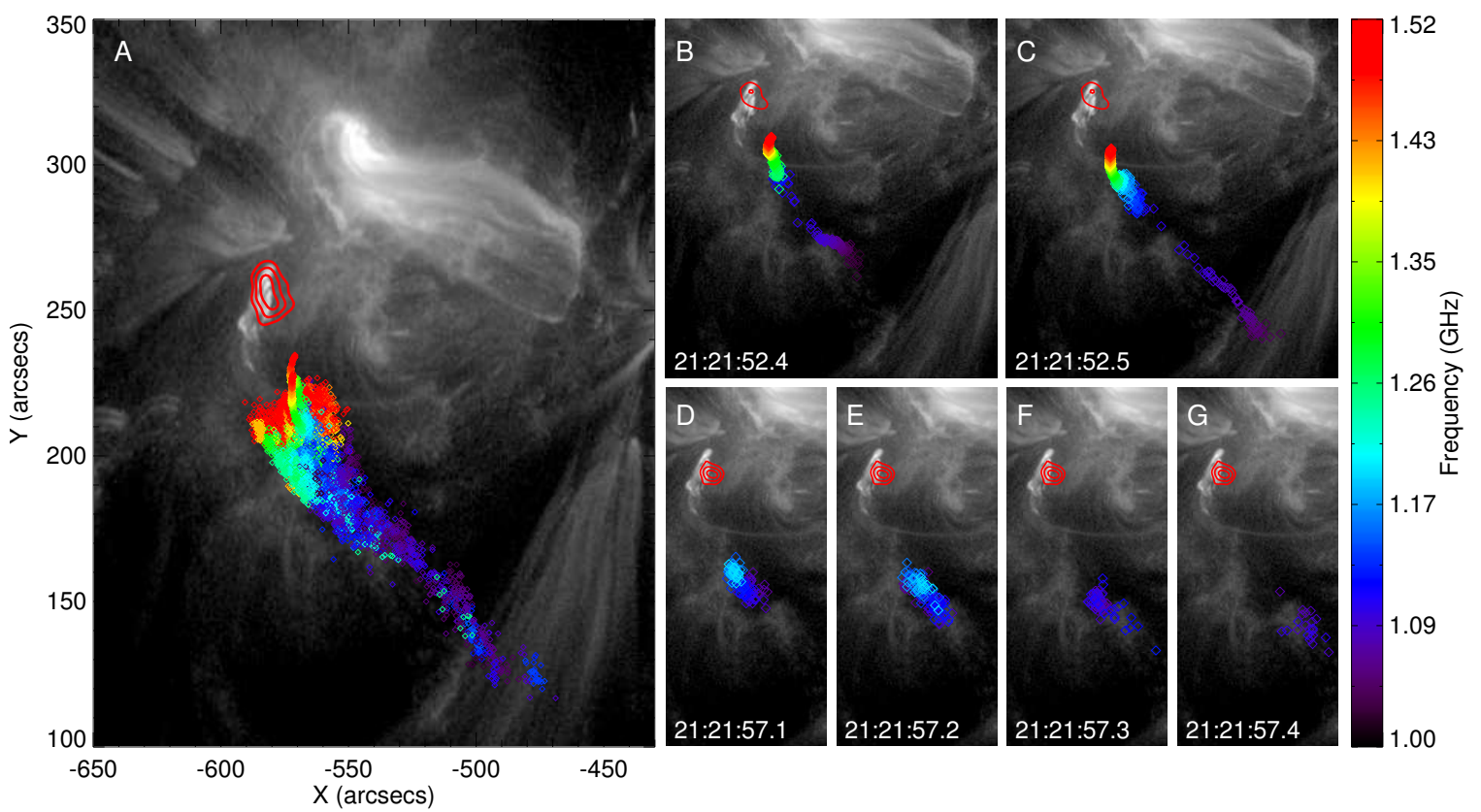

Fig. 4.4.- (A) Emission centroids of all type IIIdm bursts observed from 21:20:30 to 21:22:10 UT, colored from blue to red in increasing frequencies, showing electron beam trajectories in projection. Background is the SDO/AIA $131 \AA$ image at 21:22:09 UT. Red contours are the $12 \mathrm{~s}$ integrated $12-25 \mathrm{keV}$ HXR emission during the second HXR peak (around 21:21:50 UT). Emission centroids of a temporally resolved type IIIdm burst observed from 21:21:52.4 to 21:21:52.6 UT (shown by arrow "1" in Figure 4.2(H)) are shown by $(\mathrm{B}-\mathrm{C})$ for two successive 100-ms integrations. Another temporally-resolved type IIIdm burst from 21:21:57.1 to 21:21:57.5 UT (arrow "2" in Figure 4.2(H)) is shown by (D-G) for four successive $100 \mathrm{~ms}$ integrations. Red contours are the $12-25 \mathrm{keV}$ HXR source with a $4 \mathrm{~s}$ integration closest to the type-IIIdm-burst times.

type-IIIdm-burst sources; the latter is estimated to be $\approx 15 \mathrm{Mm}$ as we discuss further below. This energy release event was likely associated with evolving satellite magnetic polarities in AR 11339 (Figures 4.5(A)-(D)). They coincided spatially with the HXR footpoint source (Figures 4.5(A)-(D)) and the base of the EUV jet (Figures 4.5(E)-(H)). The evolution of the HXR source and the EUV jet suggests that energy release and plasma heating took place in two stages: first, an HXR footpoint source appeared over the northern satellite polarity (Figure 4.5(A)) during the first HXR 
enhancement around 21:21:00 UT, while an EUV jet was initiated (Figure 4.5(F); the timing was estimated within the 12 s cadence of the SDO/AIA images) that involved eruption of an arcade-shaped feature. During the second HXR enhancement, which peaked around 21:21:50 UT, the HXR footpoint source moved gradually to the southern satellite polarity (Figures 4.5(B)-(D)), while another eruptive EUV jet was initiated (Figures 4.5(G)-(H)). These multi-wavelength observations allow us to propose a self-consistent scenario describing the physical processes that occurred during this event (Figure 4.6). First, interaction of the satellite magnetic polarities with the surrounding magnetic flux triggered magnetic reconnection above the northern neutral line around the first HXR peak (indicated by the "X" symbol). Both upward- and downward-propagating electron beams were produced, resulting in the observed type IIIdm bursts and HXR footpoint emission (the shaded area). The released magnetic energy can trigger instabilities and initiate "blowout" EUV jets that involve eruption of a magnetic arcade (Moore et al., 2010). Magnetic field lines were stretched and led to further reconnections above the southern magnetic neutral line as well. The accelerated particles led to subsequent brightenings of the southern EUV loops, HXR footpoints, and type IIIdm bursts.

The $V L A$ data can be used to derive the electron number density $n_{e}$ along the type-IIIdm-emitting loops since it is directly related to the plasma frequency or its harmonic. We assume that the emission is likely harmonic plasma radiation for two reasons. First, fundamental plasma radiation tends to be more highly circularly polarized than its harmonic (Dulk \& Suzuki, 1980). Yet the majority of the observed type IIIdm bursts is weakly polarized $(\lesssim 20 \%)$. Second, fundamental plasma radiation results in a group delay of $\gtrsim 0.5 \mathrm{~s}$ in its transit to Earth compared to HXR photons, while the delay is expected to be far less for harmonic radiation (Dennis et al., 1984). 

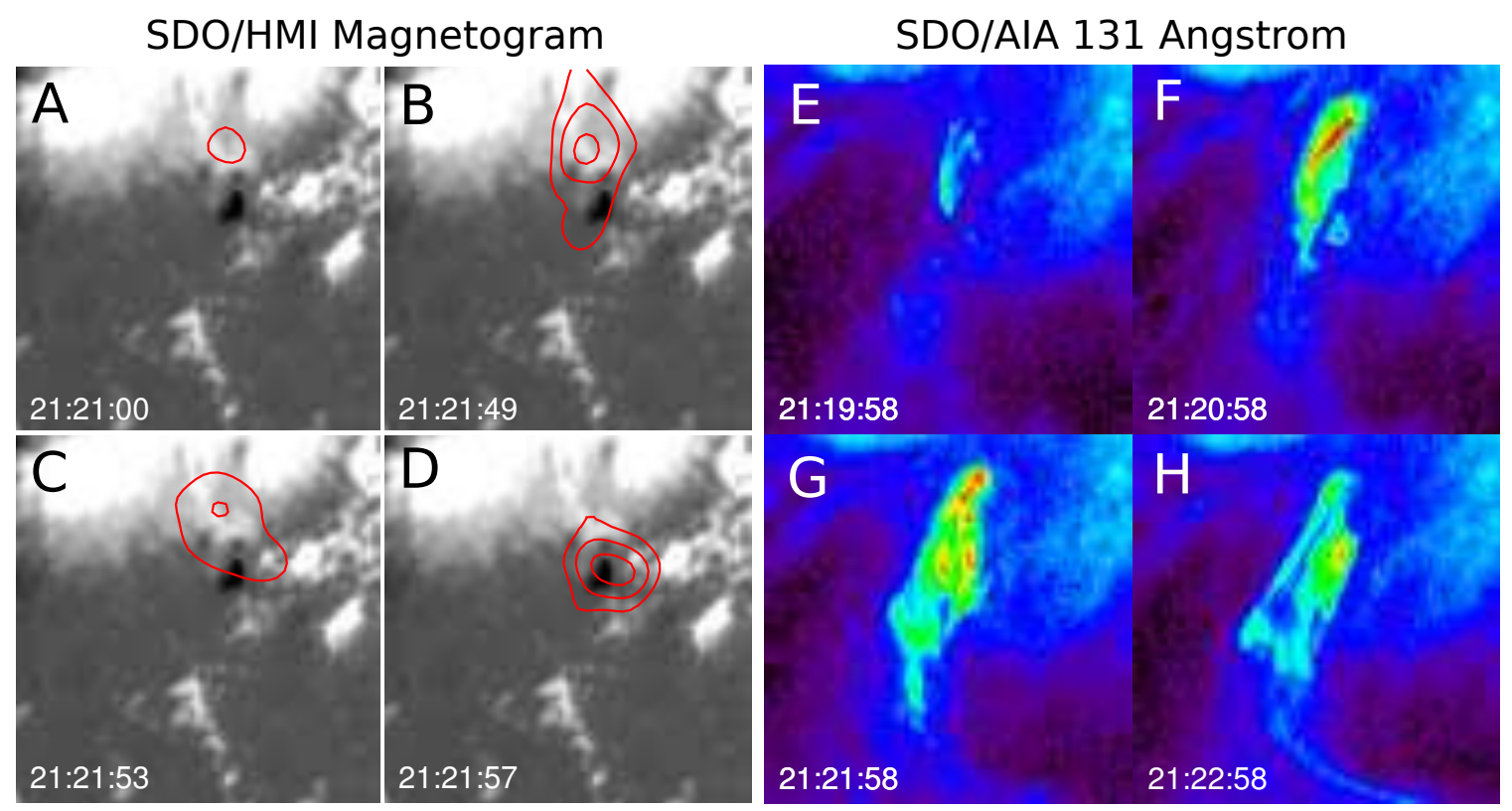

Fig. 4.5.- (A-D): A series of 12-25 keV HXR emission (red contours) overlaid on the SDO Helioseismic and Magnetic Imager (HMI; Scherrer et al., 2012) magnetogram observed during the two HXR peaks around 21:21:00 UT (A) and 21:21:50 UT (B-D). $(\mathrm{E}-\mathrm{H})$ : A detailed view of the coronal jet showing its temporal evolution in SDO/AIA $131 \AA$ images. The FOV of the images is indicated by the small box in Figure 4.2(A).

No such delay $\gtrsim 0.5 \mathrm{~s}$ is found when the HXR and radio data are cross-correlated. Therefore, we have $n_{e} \approx(\nu / 18 \mathrm{kHz})^{2}=3.3-7 \times 10^{9} \mathrm{~cm}^{-3}$ over the frequency of $1.0-1.5$ $\mathrm{GHz}$, distributed over a height range of 30-80 Mm for the majority of the type IIIdm source centroids. The radio source height was obtained by measuring the projected distance from the HXR footpoints along the beam trajectories. Based on an estimate of the inclination angle of the EUV jet $\left(\sim 45^{\circ}\right)$ from the simultaneous SDO/AIA and STEREO/EUVI B stereoscopic observations (Figure 4.3), and assuming a similar inclination for the type IIIdm bursts, the de-projected height is obtained. Fitting the ensemble of type IIIdm trajectories to a simple hydrostatic density model yields a best-fit density scale height $L_{n}=n_{e}\left(-d n_{e} / d h\right)^{-1} \approx 40 \mathrm{Mm}$, corresponding to a temperature of $0.8 \mathrm{MK}$ assuming hydrostatic equilibrium. Returning to the loca- 

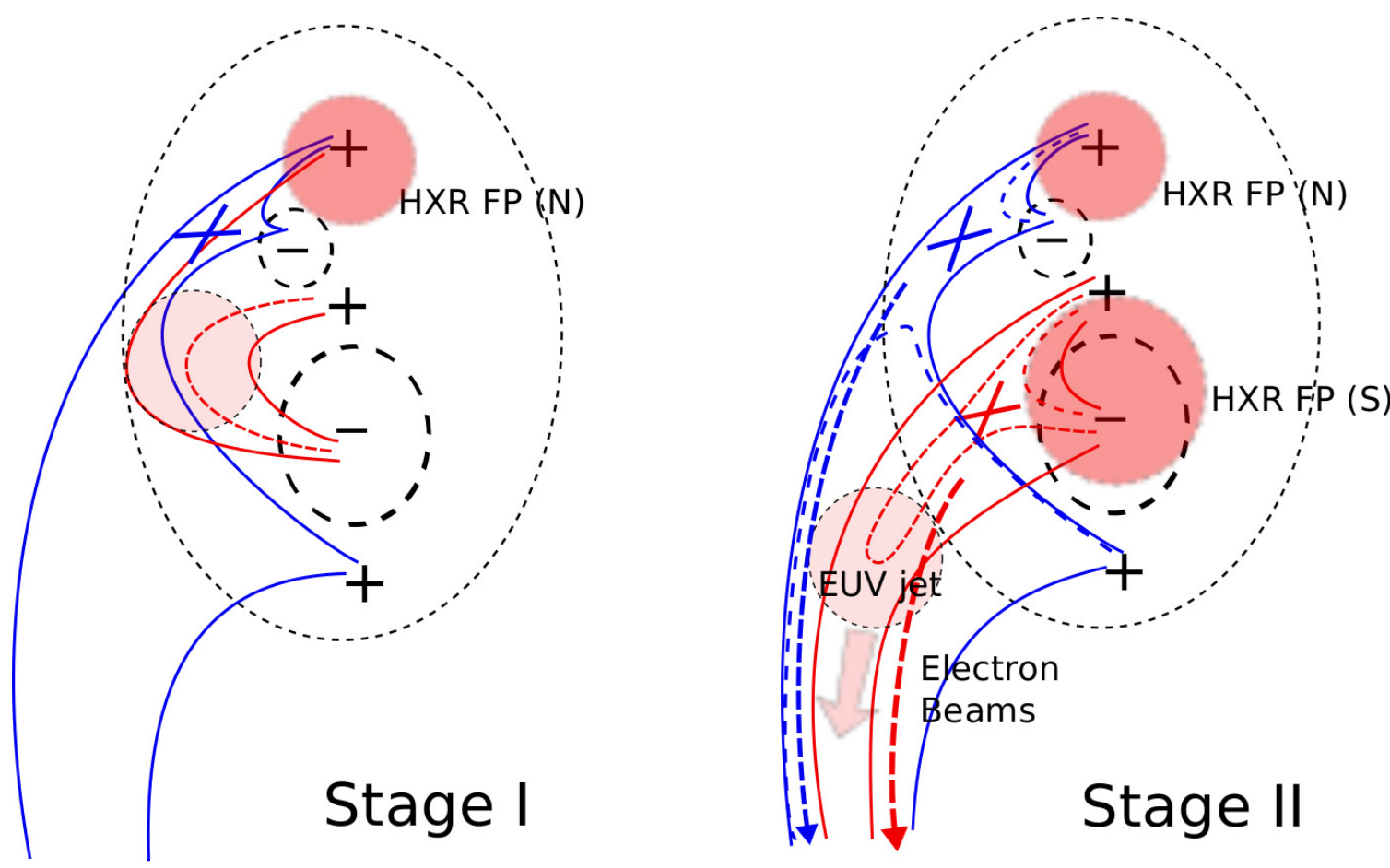

Fig. 4.6. - Schematic illustration of the magnetic field evolution, reconnection, coronal EUV jet, and generation of the electron beams and HXR footpoint sources for the impulsive energy release event. See the text for descriptions.

tion of the reconnection site, the height of the highest-frequency type-IIIdm-burst sources is found from the imaging results to be as low as $\sim 20 \mathrm{Mm}$. Considering that some bursts occurred at frequencies as high as $2 \mathrm{GHz}$, for which imaging is not available, the maximum height of the reconnection site can be reduced to $\sim 15 \mathrm{Mm}$ within the context of this density model. A striking feature of the type-IIIdm-source distributions is their frequency variation across the envelope of trajectories (Figure 4.4(A)); that is, for a given frequency the emission is not necessarily located at the same height from burst to burst, suggesting that the type-IIIdm-emitting loops are inhomogeneous perpendicular to the magnetic field, with $\Delta n_{e} /\left\langle n_{e}\right\rangle \approx 25 \%$. For the limited number of temporally resolved bursts the electron beam velocity $v_{b}$ can be directly measured from the movement of the type IIIdm source centroids in time 
(e.g., Figure 4.4(D)-(G)), which gives $v_{b} \approx 0.3 c$, where $c$ is the speed of light. This is consistent with the electron energy responsible for the observed HXR emission in the energy range $12-25 \mathrm{keV}$, further demonstrating that their parent electrons are accelerated from a common site.

Surprisingly, no trace of loop-like structures can be found along the type-IIIdmburst trajectories against the background in any of the SDO/AIA EUV filters (cf. Raulin et al., 1996), implying that the column emission measure $\xi$ of the type-IIIdmemitting loops is too small to result in detectable emission or absorption relative to the background. To quantify this, we evaluate the observed intensity of an AIA filter image $I(x, y)=\xi\left(T_{0}, x, y\right) R\left(T_{0}\right) \Delta t$, where $\xi\left(T_{0}, x, y\right)$ is the emission measure assuming an isothermal plasma temperature $T_{0}, R\left(T_{0}\right)$ is the filter response function at $T_{0}$, and $\Delta t$ is the exposure time. With the estimated temperature $(0.8 \mathrm{MK})$ of the type-IIIdm-emitting loops, the most probable EUV band to detect any signature is the AIA $171 \AA$ band. A negative result means the intensity contribution $\Delta I(x, y)$ by these loops is smaller than the rms intensity variation $\sigma_{I}$. In regions where the type IIIdm source is present we measure $\sigma_{I} \sim 40-100 \mathrm{DN}^{\text {pixel }}{ }^{-1}$ for AIA $171 \AA$ images with $\Delta t=2 \mathrm{~s}$. With $R_{171} \approx 10^{-24} \mathrm{DN} \mathrm{cm}^{5} \mathrm{~s}^{-1}$ pixel $^{-1}$ at $0.8 \mathrm{MK}$ (Lemen et al., 2012), the upper limit of the emission measure of these loops is estimated to be $\xi_{\max }=\sigma_{I} / \Delta t / R_{171}<5 \times 10^{25} \mathrm{~cm}^{-5}$. With $\xi=n_{e}^{2} V / w^{2}=n_{e}^{2} N d^{2} / w$, where $V$ is the total volume of $N$ identical loops along the line of sight within the instrument resolution element of size $w$ ( $0.4 \mathrm{Mm}$ for AIA), the upper limit of the loop diameter is $d_{\max }=\left(\xi_{\max } w / n_{e}^{2}\right)^{1 / 2} / N^{1 / 2}=l / N^{1 / 2}$. For a density of $n_{e}=3.3-7 \times 10^{9} \mathrm{~cm}^{-3}$ inside the loops, $l$ is found to be only a few $\times 10$ to $\approx 100 \mathrm{~km}$ under rather generous assumptions, and $d_{\max }$ would be even smaller with a filling factor $1 / N<1$.

The background plasma density and temperature can be estimated by utilizing 
the observed background intensities $I_{b}(x, y)$ from all the AIA EUV bands sensitive to a wide range of coronal temperature. We use Aschwanden et al. (2013)'s automated forward-fitting technique to obtain a differential emission measure distribution, $d \xi_{b}(T, x, y) / d T$, which yields an integrated emission measure of $\xi_{b} \approx 5 \times 10^{27}$ $\mathrm{cm}^{-5}$ peaking at $\sim 2.8 \mathrm{MK}$. With $\xi_{b}=\int n_{e}(h)^{2} d h$, the background density is found to vary from $6.9 \times 10^{8}$ to $4.8 \times 10^{8} \mathrm{~cm}^{-3}$ over $30-80 \mathrm{Mm}$ at which the type IIIdm bursts occur. Hence, the type-IIIdm-emitting loops may be cooler (by a factor of 3 ) and denser (by roughly an order of magnitude) relative to the background coronal medium. This is consistent with the scenario proposed by Benz et al. (1992) for allowing the type IIIdm emission to escape. The over-density is also consistent with past conclusions based on the observed contrast between $\xi$ toward bright EUV/SXR loops and $\xi_{b}$ toward the background corona (Hara et al., 1992; Aschwanden, 2001), which suggests that the relevant active region loops may have already been supplied with high-density chromospheric plasmas through previous heating processes.

The existence of ultra-fine magnetic structures in the corona has been previously suspected (see, e.g., Reale 2010, and references therein) but no current instrumentation can resolve such structures at coronal temperatures. Recent observations of ultra-fine magnetic structures have been of plasma at chromospheric temperatures (Ji et al., 2012; Antolin \& Rouppe van der Voort, 2012), reporting loop diameters ranging from $\sim 100$ to a few $\times 100 \mathrm{~km}$. Our observations suggest the corona is "fibrous" in nature, consisting of many unresolved "strands". It should also be noted that the type-IIIdm-emitting strands have a direct connectivity to the reconnection region. Thus these strands should be even thinner down at the reconnection region with a diameter of at most $10 \mathrm{~s}$ of $\mathrm{km}$ due to their general expansion with increasing coronal height (DeForest, 2007). Furthermore, the multitudes of discrete electrons beams 
observed have access to spatially distinct coronal magnetic strands in $\lesssim 1$ s (Figure 4.4(A)), which indicates that the reconnection region likely consists of a large number of discrete reconnection sites in a localized spatial volume. Our observations rather directly suggest a bursty reconnection scenario involving a localized reconnection region containing a distribution of many small-scale dynamically evolving structures for magnetic energy release and particle acceleration. This picture is qualitatively consistent with previous work on fragmentary energy release on the Sun (see, e.g., van den Oord 1994, and references therein) as well as more recent developments in the physics of magnetic reconnection, such as experiments in the laboratory (Moser \& Bellan, 2012) and numerical simulations (Kliem et al., 2000; Drake et al., 2006; Karlický \& Bárta, 2007), which show the reconnection region consists of many "magnetic islands" or "filaments" developed through magnetohydrodynamic instabilities such as tearing or kink instability. The constraints in the temporal and spatial scales of fragmentations we report here and in future observations of this kind may help guide theoretical reconnection models as well as constrain parametric inputs to numerical simulations.

\subsection{Conclusion}

Dynamic imaging spectroscopy of type IIIdm bursts with the $V L A$ has allowed us to map the trajectories of electron beams produced by magnetic energy release during a coronal jet that occurred in the aftermath of a flare. Electrons escaped along discrete, ultra-fine strands into the upper atmosphere, producing the observed type IIIdm bursts. Together with magnetic, EUV, and HXR data, we showed that the energy release site was associated with a coronal jet and that the magnetic reconnection process involved different locations at different times and that, furthermore, the 
density at each location varied significantly. The spatial scales in the reconnection sites are likely $10 \mathrm{~s}$ of $\mathrm{km}$ or less. We conclude that the magnetic energy release process is highly fragmentary and that the surrounding coronal medium is fibrous in nature. 


\section{Chapter 5}

\section{Hard X-Ray Sources in the}

\section{Corona: a Study on the Emission} Mechanisms*

*The major content is adapted from Chen \& Bastian (2012). Tim Bastian came up with the initial idea. I did the majority of the analytical derivations, numerical calculations, scientific analysis, and writing. Tim Bastian worked closely with me throughout the paper and contributed to the writing and revision. We also received helpful comments from an anonymous referee, which led to an improved discussion of the inverse Compton scattering in comparison with the thin-target non-thermal bremsstrahlung emission. 


\subsection{Introduction}

Solar flares produce HXR and continuum $\gamma$-ray emission, generally attributed to thermal or non-thermal bremsstrahlung emission. As such, HXR emission provides key diagnostics of plasma heating, electron acceleration, and electron transport. Intense chromospheric HXR thick target emission is produced at the footpoints of coronal magnetic loops. The relatively faint coronal HXR and continuum $\gamma$-ray emission is generally more difficult to observe in the presence of intense footpoint emission given the limited dynamic range of X-ray imaging instruments. In most cases, therefore, coronal HXR or $\gamma$-ray emission is observed in flares that occur in active regions behind the solar limb; the intense footpoint emission is occulted, thereby revealing the relatively faint coronal emission. While reports of coronal HXR emission data back to the early 1970s (e.g., Frost \& Dennis, 1971), imaging observations, first by Yohkoh (Kosugi et al., 1992) and then by the Ramaty High-Energy Solar Spectroscopic Imager (RHESSI; Lin et al., 2002), have led to renewed interest in coronal HXR sources.

As discussed in the review by Krucker et al. (2008b), coronal HXR sources reveal a diverse phenomenology. These include sources that precede the impulsive phase (Lin et al., 2003) as well as a variety of coronal HXR sources that may occur during the impulsive phase: "over-the-loop-top" sources (Masuda et al., 1994), double sources (Sui \& Holman, 2003), and coronal thick target sources (Veronig \& Brown, 2004). During the late phase of flares "superhot" thermal sources (Lin et al., 1981), gradual sources that display a "soft-hard-harder" spectral evolution (Cliver et al., 1986; Kiplinger, 1995), and non-thermal sources that display hard, continuum $\gamma$-ray emission (Krucker et al., 2008a) may occur. Coronal HXR sources have been observed over a range of heights in the corona and can be associated with stationary or moving sources. We refer the reader to Krucker et al. (2008b), and references therein, for a 
more detailed discussion of the types of coronal HXR sources, their properties, and the circumstances under which they occur.

We focus here on non-thermal coronal HXR and continuum $\gamma$-ray sources that occur during the impulsive phase of flares. These have been interpreted in terms of thin-target, non-thermal bremsstrahlung. This may well be correct but in the case of certain coronal HXR, or continuum $\gamma$-ray, sources the parameters required can be extreme. For example, three powerful X-class flares - those on 2003 October 28, 2005 January 20, and 2005 September 7 - were accompanied by continuum $\gamma$-ray emission $>200 \mathrm{keV}$ (Krucker et al., 2008a). These flares, observed by RHESSI, were not occulted by the limb and both footpoint and coronal emissions were observed. In the case of 2005 January 20 footpoint emission dominated during times near the $\gamma$-ray maximum but the coronal source became increasingly prominent during the decay of the $\gamma$-ray emission. The power-law index $\alpha$ of the photon spectrum of the coronal source from $200-800 \mathrm{keV}$ was significantly harder $(\alpha \approx 1.5)$ than that of the footpoints $(\alpha \approx 2.9)$. The other two flares displayed similar properties. Interpreting the emission in terms of non-thermal, thin-target, electron-ion bremsstrahlung emission implies that the spectral indices of the coronal $\gamma$-ray sources were near the minimum values theoretically possible and require the effective low-energy cutoff of the energetic electrons responsible for the emission to have been $>1 \mathrm{MeV}$ in all cases (Brown et al., 2008). Another intriguing example is the observation of an HXR source high in the corona (Krucker et al., 2007b). The flare itself occurred $40^{\circ}$ behind the limb; to be visible from the Earth the HXR source was at a radial height of order $150 \mathrm{Mm}$ where the ambient density was estimated to be only $\sim 10^{8} \mathrm{~cm}^{-3}$. In order to produce the diffuse HXR source via thin-target bremsstrahlung roughly $10 \%$ of the electrons were accelerated. Yet more extreme is an event recently reported by Krucker et al. (2010) 
that bears a resemblance to the celebrated "Masuda flare" (Masuda et al., 1994). Also observed by RHESSI, the limb-occulted flare on 2007 December 31 showed a relatively intense nonthermal coronal HXR source located $\approx 6 \mathrm{Mm}$ above the thermal flare loops. The HXR source can be understood in terms of non-thermal, thin-target bremsstrahlung emission from a power-law distribution of electrons if essentially all of the electrons in the source are accelerated to nonthermal energies. It is worth asking whether an alternate emission mechanism is responsible for, or may contribute to, some coronal HXR sources.

In considering plausible mechanisms for HXR emission from solar flares, Korchak (1971) considered non-thermal bremsstrahlung emission, synchrotron emission, and inverse Compton scattering (ICS). He concluded that bremsstrahlung emission is favored in most cases but that ICS could play a role under some circumstances - if the ambient density in the source is low, for example. Synchrotron radiation is not favored as a mechanism for HXR emission from flares. In light of the many recent observations of coronal HXR sources, however, it seems timely to revisit the question of whether ICS plays a role. We are not the first to do so. Motivated in part by the observations cited above, MacKinnon \& Mallik (2010) recently considered whether photospheric photons up-scattered to HXR or $\gamma$-ray energies by relativistic electrons or positrons by ICS could account for coronal HXR sources. They consider scattering of the (anisotropic) photospheric photon field on an isotropic, power-law distribution of electrons from sources at various heliographic longitudes. They find that conditions for ICS are most favorable for coronal sources near the limb provided that the ambient density is sufficiently low. They also find that relatively modest numbers of energetic electrons are required for ICS to account for the continuum $\gamma$-ray sources discussed by Krucker et al. (2008a). Unfortunately, the work contained an error that renders 
their estimates of the number density of energetic electrons required too optimistic.

In this paper, we correct and expand upon the work of MacKinnon \& Mallik (2010, hereafter MM10). Unlike MM10, we do not consider ICS on positrons. We begin by considering, as they do, the case of ICS from an isotropic, power-law distribution of ultra-relativistic electrons scattering an anisotropic field of photons (Section 5.2.1, first part). Noting that flares produce copious EUV and SXR emission, we next consider the case where EUV/SXR photons are up-scattered to HXR or $\gamma$-ray energies by a power-law distribution of mildly relativistic electrons (Section 5.2.1, second part). To do so requires evaluating exact expressions for the ICS photon scattering rate. These calculations show that the ICS spectrum resulting from scattering on mildly relativistic electrons has steeper photon spectrum than that resulting from scattering on ultra-relativistic electrons. Finally, we note that anisotropic electron distributions may have significant implications for ICS. We consider ICS on "beam" and "pancake" electron distributions in Section 5.2.2, showing that they can result in significant enhancements compared with ICS on isotropic electrons with the same number density for favorable viewing geometries. We briefly review results from anisotropic electronion and electron-electron bremsstrahlung in Section 5.3 and reconsider circumstances under which non-thermal bremsstrahlung or ICS is favored. We discuss our results in light of selected observations of coronal HXR/ $\gamma$-ray sources in Section 5.4 and conclude in Section 5.5. 


\subsection{Inverse Compton Scattering Emission}

\subsubsection{ICS of Photons on an Isotropic Electron Distribution}

In this section we consider ICS of isotropic and anisotropic photon distributions on isotropic distributions of ultra-relativistic electrons (Lorentz factor $\gamma \gg 1$ ) and mildly relativistic electrons $(\gamma \sim 2-10)$. The vast majority of studies that consider ICS do so for astrophysical regimes where the electrons in question are ultra-relativistic and the ambient plasma density is very low. In the ultra-relativistic case, certain approximations can be made that greatly simplify the relevant expressions for the photon distribution function or emissivity. We therefore begin by considering ultrarelativistic electrons interacting with a photon field. We take $\hbar=m_{e}=c=1$ throughout the paper.

\section{Ultrarelativistic Regime}

Jones (1968) first derived exact expressions for ICS from an isotropic distribution of ultrarelativistic monoenergetic electrons interacting with an isotropic distribution of monoenergetic photons normalized to one electron passing through a photon field of unit number density. The net rate at which photons are scattered into a particular energy $\epsilon_{2}$ is given by

$$
R_{\mathrm{iso}}\left(\epsilon_{2}\right)=\int d \gamma n_{e}(\gamma) \int d \epsilon_{1} n_{\gamma}\left(\epsilon_{1}\right) \frac{d R_{\mathrm{iso}}\left(\gamma, \epsilon_{1}\right)}{d \epsilon_{2}}
$$

where $n_{e}(\gamma)$ is the electron number density distribution, $n_{\gamma}\left(\epsilon_{1}\right)$ is the number density distribution of the incident photons, and $d R_{\text {iso }}\left(\epsilon_{1}\right) / d \epsilon_{2}$ is the rate at which photons are scattered from $\epsilon_{1}$ to $\epsilon_{2}$ (note that $\epsilon_{2}$ may be greater than or less than $\epsilon_{1}$, in general). The time dependence is implicit. 
The exact expressions for the differential scattering rate are rather cumbersome although they have been presented in simplified form by Pe'er \& Waxman (2005). Jones shows that the exact expressions for the differential rates at which photons are up-scattered or downscattered can be approximated by the much simpler expressions:

$$
\frac{d R_{\mathrm{iso}}\left(\gamma, \epsilon_{1}\right)}{d \epsilon_{2}}=\frac{2 \pi r_{e}^{2}}{\epsilon_{1} \gamma^{2}}\left[2 q \ln q+(1+2 q)(1-q)+\frac{1}{2} \frac{\left(4 \epsilon_{1} \gamma q\right)^{2}}{\left(1+4 \epsilon_{1} \gamma q\right)}(1-q)\right] \quad\left(\epsilon_{2}>\epsilon_{1}\right)
$$

$$
\frac{d R_{\text {iso }}\left(\gamma, \epsilon_{1}\right)}{d \epsilon_{2}}=\frac{\pi r_{e}^{2}}{2 \epsilon_{1} \gamma^{4}}\left[\left(q^{\prime}-1\right)\left(1+2 / q^{\prime}\right)-2 \ln q^{\prime}\right] \quad\left(\epsilon_{2}<\epsilon_{1}\right)
$$

where $q=\epsilon_{2} /\left[4 \epsilon_{1} \gamma^{2}\left(1-\epsilon_{2} / \gamma\right)\right]$, with $1 /\left(4 \gamma^{2}\right)<q \leq 1$; and $q^{\prime}=4 \gamma^{2} \epsilon_{2} / \epsilon_{1}$. Henceforth, we ignore the case where $\epsilon_{2}<\epsilon_{1}$. Jones (1968) and Blumenthal \& Gould (1970) demonstrate that for an isotropic distribution of electrons with a power-law distribution of energy and an index $\delta$ - that is, $f(\gamma) \sim \gamma^{-\delta}$ - the resulting spectrum of upscattered photons is itself a power law with an index $(\delta+1) / 2$. Moreover, the upscattered spectrum is insensitive to the details of the incident photon spectrum. In fact, the energy distribution of the incident photons $\epsilon_{1}$ may be approximated as a $\delta$-function when $\epsilon_{2} / \epsilon_{1} \gg 1$.

For the particular case of ICS on the Sun, we consider, as have previous authors, the case of ultra-relativistic electrons, presumably accelerated by a flare, interacting with soft photospheric photons. Clearly, the photospheric photon field is not isotropic. It is convenient to use the results of Moskalenko \& Strong (2000), who derived the differential distribution of upscattered photons for the more general case of anisotropic photons scattering off of an isotropic electron distribution. They find that for an 
isotropic distribution of monoenergetic electrons described by

$$
f_{e}\left(\gamma_{e}, \Omega_{e}\right)=\frac{1}{4 \pi \gamma_{e}^{2}} \delta\left(\gamma_{e}-\gamma\right)
$$

and monoenergetic incident photons with a distribution

$$
f_{\gamma}\left(\epsilon_{\gamma}, \Omega_{\gamma}\right)=Q_{\gamma}\left(\Omega_{\gamma}\right) \frac{1}{\epsilon_{\gamma}^{2}} \delta\left(\epsilon_{\gamma}-\epsilon_{1}\right)
$$

where $Q_{\gamma}\left(\Omega_{\gamma}\right)$ is the angular distribution of the photons, the upscattered photon distribution is given by

$$
\begin{aligned}
\frac{d R(\gamma)}{d \epsilon_{2}}= & \frac{\pi r_{e}^{2}}{\epsilon_{1}\left(\gamma-\epsilon_{2}\right)^{2}} \int_{\Omega_{\gamma}} d \Omega_{\gamma} Q_{\gamma}\left(\Omega_{\gamma}\right) \\
& \times\left[2-2 \frac{\epsilon_{2}}{\gamma}\left(\frac{1}{\epsilon_{1}^{\prime}}+2\right)+\frac{\epsilon_{2}^{2}}{\gamma^{2}}\left(\frac{1}{\epsilon_{1}^{\prime 2}}+\frac{2}{\epsilon_{1}^{\prime}}+3\right)-\frac{\epsilon_{2}^{3}}{\gamma^{3}}\right],
\end{aligned}
$$

where

$$
\epsilon_{2} \leq 2 \gamma \epsilon_{1}^{\prime} /\left(1+2 \epsilon_{1}^{\prime}\right), \quad \epsilon_{1}^{\prime}=\epsilon_{1} \gamma(1+\cos \zeta)
$$

and $\zeta$ is the angle between the momenta of the electron and the incident photon. We have $\zeta=0$ for a head-on collision from which the maximum energy of the upscattered photon results

$$
\epsilon_{2}{ }^{\max }=\frac{4 \epsilon_{1} \gamma^{2}}{1+4 \epsilon_{1} \gamma} \approx 4 \epsilon_{1} \gamma^{2}
$$

If we assume a fully isotropic photon distribution, i.e., $Q_{\gamma}(\theta, \phi)=1 /(4 \pi)$, it can be shown (Moskalenko \& Strong, 2000) that Equation (5.6) simplifies to the approximate formula of Jones (1968) given above by Equation (5.2).

To make further progress we adopt the geometry employed by MM10, shown in Figure 5.1. The $Z$-axis is normal to the solar surface (the $X-Y$ plane); $\hat{\gamma}$ is the 


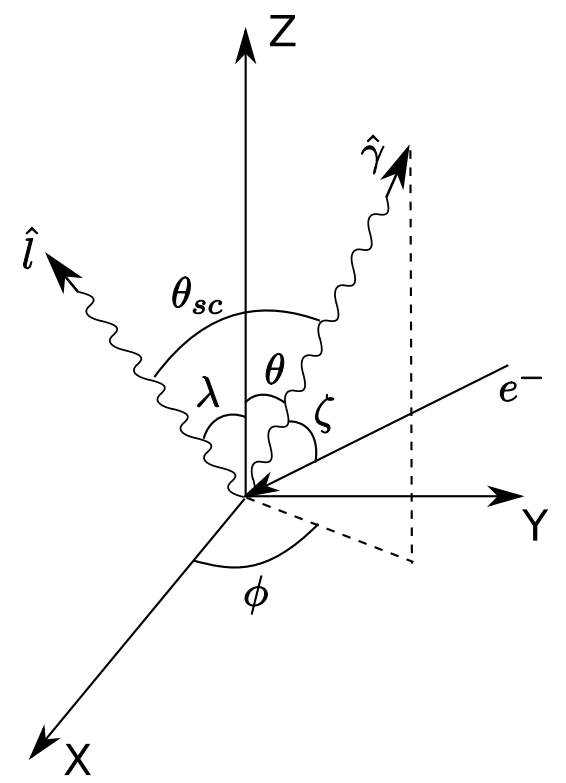

Fig. 5.1.- Geometry and angles used to calculate the ICS emission from an anisotropic photon field. The $X-Y$ plane is the solar surface; the $Z$-axis is normal to the solar surface; $\hat{\gamma}$ is the unit vector along the incident photons, with $\theta$ and $\phi$ as the polar and azimuthal angles; the $X$-axis is chosen such that the unit vector along the line of sight (LOS) to the observer $\hat{l}$ lies in the $X-Z$ plane; $\lambda$ is the heliocentric angle of the source location; $\theta_{\mathrm{sc}}$ is the scattering angle between the incident and up-scattered photons; $\zeta$ is the angle between the electrons and the incident photons.

unit vector along the direction of the incident photons, with $\theta$ and $\phi$ as the polar and azimuthal angles; The $X$-axis is defined to place the unit vector along the line of sight (LOS) to the observer $\hat{l}$ in the $X-Z$ plane, with $\lambda$ the angle between the $Z$-axis and the LOS (the heliocentric angle of the source from the disk center). The scattering angle between the incident photon and the up-scattered photon is given by $\theta_{\mathrm{sc}}$. The angle $\zeta$ in the integrand of Equation (5.6) through Equation (5.7) complicates the integration somewhat. Noting that, for ultrarelativistic electrons with $\gamma \gg 1$, incident photons are upscattered into narrow cone of angular width $\sim 1 / \gamma$ in the direction of the energetic electrons, we can approximate the upscattered photons to be unidirectional which, in turn, allows us to approximate the angle $\zeta$ in Equation (5.7) as $\zeta \simeq \pi-\theta_{\text {sc }}$. 
It is easy to show from the geometry in Figure 5.1 that $\theta_{\mathrm{sc}}$ is in fact a function of $\theta$, $\phi$, and $\lambda$ :

$$
\cos \theta_{\mathrm{sc}}=\cos \theta \cos \lambda+\sin \theta \sin \lambda \cos \phi
$$

It is also seen that Equation (5.7) requires $\gamma \geq \gamma_{\min }=(1 / 2) \sqrt{\epsilon_{2} / \epsilon_{1}}$ in the ultrarelativistic limit. Given the photon distribution function $Q_{\gamma}\left(\Omega_{\gamma}\right)=Q_{\gamma}(\theta, \phi)$, the integration of Equation (5.6) becomes straightforward.

Following MM10, the angular distribution of photospheric photons is taken to fill the half-space above the photosphere (see Figure 5.2) and the photon angular distribution is expressed simply as

$$
Q_{\gamma}(\theta, \phi)=\frac{1}{2 \pi} H\left(\frac{\pi}{2}-\theta\right)
$$

where $H$ is the Heaviside function. However, in their subsequent derivation MM10 make an error in the expression for the photon emissivity (their Equation (5)) that leads to the inclusion of a factor $1+\cos \theta$ rather than $1-\cos \theta_{\text {sc }}$. In fact, after substituting the expressions for $Q_{\gamma}(\theta, \phi)$ and $\epsilon_{1}^{\prime}$ into Equation (5.6), the expression should read

$$
\begin{aligned}
\frac{d R(\gamma, \lambda)}{d \epsilon_{2}}= & \frac{r_{e}^{2}}{2 \epsilon_{1}\left(\gamma-\epsilon_{2}\right)^{2}}\left[\left(2-\frac{4 \epsilon_{2}}{\gamma}+\frac{3 \epsilon_{2}^{2}}{\gamma^{2}}-\frac{\epsilon_{2}^{3}}{\gamma^{3}}\right) \int_{0}^{2 \pi} d \phi \int_{0}^{\pi / 2} \sin \theta d \theta\right. \\
& -\frac{1}{\epsilon_{1} \gamma}\left(\frac{2 \epsilon_{2}^{2}}{\gamma^{2}}-\frac{2 \epsilon_{2}}{\gamma}\right) \int_{0}^{2 \pi} d \phi \int_{0}^{\pi / 2} \frac{d \cos \theta}{1-\cos \theta_{\mathrm{sc}}}, \\
& \left.-\frac{\epsilon_{2}^{2}}{\epsilon_{1}^{2} \gamma^{4}} \int_{0}^{2 \pi} d \phi \int_{0}^{\pi / 2} \frac{d \cos \theta}{\left(1-\cos \theta_{\mathrm{sc}}\right)^{2}}\right]
\end{aligned}
$$

where the scattering angle $\theta_{\mathrm{sc}}$ is given by Equation (5.9). The integration limits over $\theta$ also differ from those employed by MM10. Note, too, the kinematic restriction on 
the scattering angle $\theta_{\text {sc }}$ imposed by Equation (5.7):

$$
\cos \theta_{\mathrm{sc}} \leq 1-\frac{\epsilon_{2}}{2 \epsilon_{1} \gamma\left(\gamma-\epsilon_{2}\right)}
$$

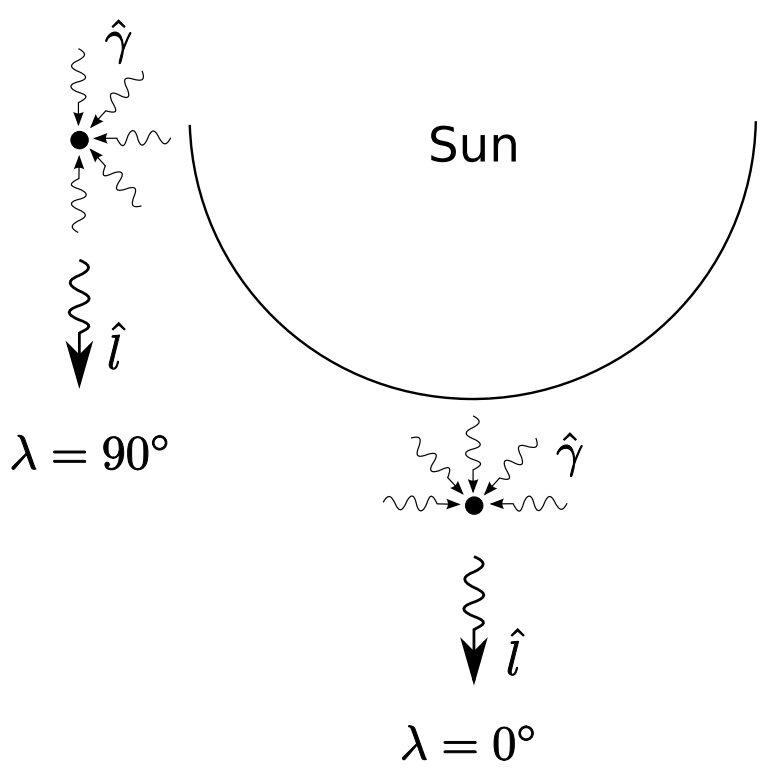

Fig. 5.2.- Illustration of the ICS emission for the incident photon distribution in association with the source geometry assumed in MM10.

We have calculated ICS spectra numerically using Equation (5.11). The photon emissivity spectrum (photons $\mathrm{cm}^{-3} \mathrm{~s}^{-1} \mathrm{sr}^{-1} \mathrm{keV}^{-1}$ ) is obtained by integration over the electron and photon energy distributions with suitable normalization. For the purposes of comparison, we use the same parameters as MM10: the incident photons are assumed to be photospheric, with an energy $\epsilon_{1}=2 \mathrm{eV}$ and a number density $n_{\gamma}=10^{12} \mathrm{~cm}^{-3}$; the electron kinetic energy is assumed to have a power-law form, $f(\gamma) \sim(\gamma-1)^{-\delta}$. In the left panel of Figure 5.3, we show the ICS photon spectra 
resulting from an electron distribution extending to $100 \mathrm{MeV}$ with a spectral index $\delta=3$, viewed with angles ranging from $\lambda=0$ (disk center) to $\lambda=2 \pi / 3$ (over the limb). The results are normalized such that $n_{e}(\gamma)=1$ electron $\mathrm{cm}^{-3}$ with an energy $>0.5 \mathrm{MeV}(\gamma>2)$. The right panel of Figure 5.3 shows the ICS spectra from electron distributions with different values of the spectral index $\delta$, for a source on the solar $\operatorname{limb}(\lambda=\pi / 2)$.
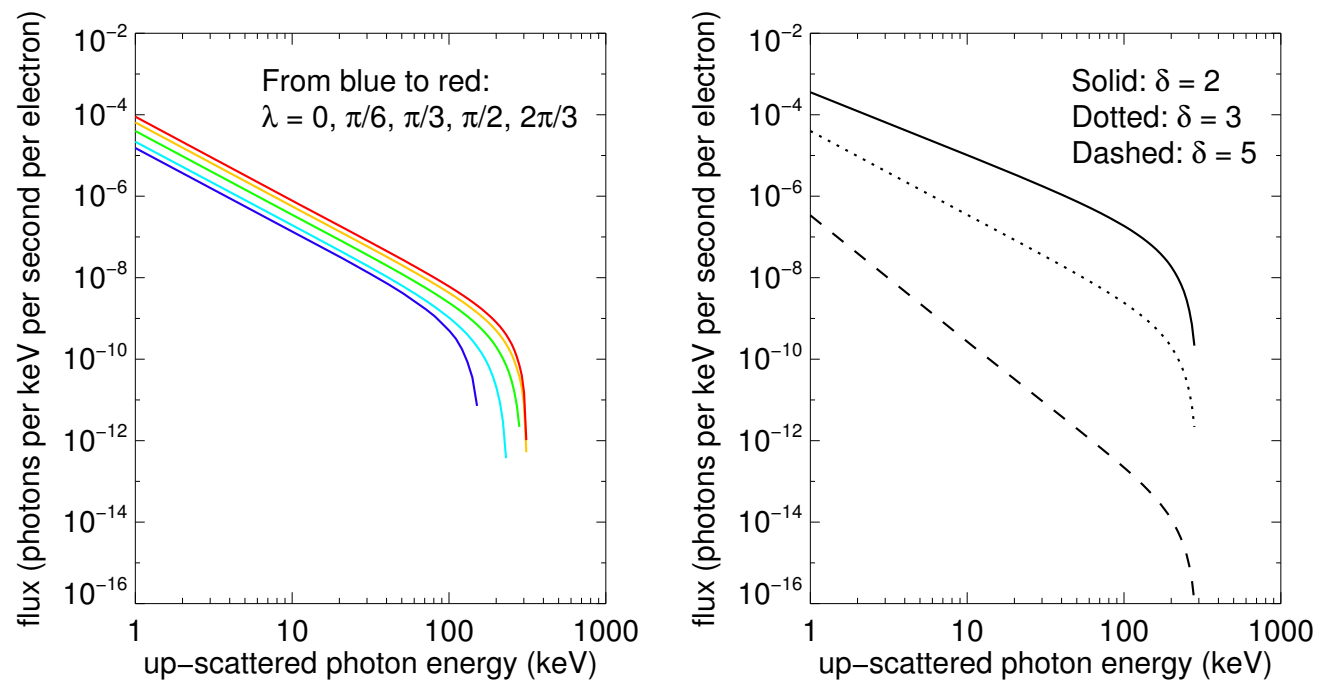

Fig. 5.3.- Left: photon flux at the Sun (photons per keV per second per electron) when the source is at the disk center $\left(\lambda=0\right.$; blue), at a longitude of $30^{\circ}(\lambda=\pi / 6)$, $60^{\circ}(\lambda=\pi / 3), 90^{\circ}(\lambda=\pi / 2)$, and over the limb at $120^{\circ}(\lambda=2 \pi / 3$; red $)$. The electron energy spectral index is $\delta=3$. Right: photon flux at the Sun (photons per $\mathrm{keV}$ per second per electron) for a source on the limb for the electron energy distribution function with different spectral indices: solid is for $\delta=2$, dotted for $\delta=3$, and dashed for $\delta=5$. Both panels have an incident photon energy of $2 \mathrm{eV}$ with a number density of $10^{12} \mathrm{~cm}^{-3}$.

We find that the calculated HXR spectra have a photon spectral index of $\alpha \simeq$ $(\delta+1) / 2$, as expected for the ultrarelativistic case (Jones, 1968; Blumenthal \& Gould, 1970), similar to those obtained by MM10 (their Figure 2 and 4). However, our photon fluxes are more than two orders of magnitude lower than those reported by MM10. 
They are similar in order of magnitude to the fully-isotropic case calculated from Equation (5.2) as might be expected (Moskalenko \& Strong, 2000). We find, moreover, that the difference in the HXR photon spectra calculated for different viewing angles $\lambda$ lies within an order of magnitude of each other, in contrast to the large range of values reported by MM10 which span more than two orders of magnitude. In practice, the center-to-limb variation of an ICS source would be modified by the contribution of Compton backscatter of ICS HXR photons on photospheric electrons (Kontar et al., 2006) in the 10-100 keV energy range, an effect that we do not include. Note that the high-energy cutoff of the photon spectrum depends on viewing angle because the maximum energies from upscattering are achieved for largest scattering angles. Finally, the high-energy cutoff of the upscattered photons from different electron power-law energy distributions is independent of the spectral index $\delta$, while those reported by MM10 vary significantly with $\delta$.

We conclude that our results for ICS in the limit of ultrarelativistic electron energies are consistent with expectations. We attribute the differences between the calculations reported here and those reported by MM10 to an error made in the expression for the photon emissivity in the latter publication.

\section{Mildly Relativistic Regime}

We now explore ICS for cases in which the electrons are not necessarily highly relativistic. Solar flares produce copious EUV and SXR photons. These may be upscattered to HXR or $\gamma$-ray energies by electrons with far lower energies than generally considered by previous treatments of ICS. For example, an $\epsilon_{1}=1 \mathrm{keV}$ SXR photon can be upscattered to $\epsilon_{\max } \approx 4 \gamma^{2} \epsilon_{1}=16-100 \mathrm{keV}$ for electrons with $\gamma=2-5$. While the photon number density of EUV/SXR photons is much smaller $\left(\lesssim 10^{7}-10^{8} \mathrm{~cm}^{-3}\right)$ 
than the number density of photospheric photons $\left(\sim 10^{12} \mathrm{~cm}^{-3}\right)$, we note that given power-law, or similar, distributions inferred for electron energy distributions during solar flares, the number of mildly relativistic electrons produced by a solar flare far outnumber those at ultrarelativistic energies. The product of the photon number density and the electron number density $n_{\gamma} n_{e}$ may therefore not differ substantially between the ultrarelativistic and mildly relativistic cases.

In considering ICS on mildly relativistic electrons, however, we can no longer exploit the approximations possible for the case of ultra-relativistic electrons and we must instead use the general expression. Consider an electron distribution expressed in separable form as $f_{e}(\gamma, \Omega)=K_{e} F_{e}(\gamma) Q_{e}\left(\Omega_{e}\right)=K_{e} F_{e}(\gamma) /(4 \pi)$ for an isotropic distribution, where $K_{e}$ is a normalization factor to ensure that the integral over the electron energy distribution results in the total number density of fast electrons. The general expression for the ICS emission rate for photons with a direction $\Omega_{\gamma}$ on an isotropic electron distribution is given by Brunetti (2000, see also Aharonian \& Atoyan 1981):

$$
\begin{aligned}
\frac{d^{2} R\left(\Omega_{\gamma}\right)}{d \epsilon_{2} d \Omega_{\gamma}} & =\frac{K_{e} \pi r_{0}^{2} \epsilon_{2}}{\epsilon_{1}^{2}} \int_{\gamma} \frac{F_{e}(\gamma)}{\beta \gamma^{2}}\left\{\frac{2 \epsilon_{1}}{\left(\epsilon_{1}^{2}+\epsilon_{2}^{2}-2 \epsilon_{1} \epsilon_{2} \cos \theta_{\mathrm{sc}}\right)^{1 / 2}}\right. \\
& +\left[\epsilon_{1}\left(1-\cos \theta_{\mathrm{sc}}\right)-\frac{2}{\epsilon_{2}}-\frac{2}{\epsilon_{1} \epsilon_{2}^{2}\left(1-\cos \theta_{\mathrm{sc}}\right)}\right]\left(\frac{1}{R_{1}}-\frac{1}{R_{2}}\right) \\
& +\frac{1-\cos \theta_{\mathrm{sc}}}{\epsilon_{1} \epsilon_{2}^{2}}\left[\frac{\left(\gamma-\epsilon_{2}\right) \epsilon_{2}+\gamma \epsilon_{1}+\epsilon_{2} \epsilon_{1} \cos \theta_{\mathrm{sc}}}{R_{1}^{3}}\right. \\
& \left.\left.+\frac{\left(\gamma+\epsilon_{1}\right) \epsilon_{1}+\gamma \epsilon_{2}-\epsilon_{2} \epsilon_{1} \theta_{\mathrm{sc}}}{R_{2}^{3}}\right]\right\} d \gamma
\end{aligned}
$$

where

$$
\begin{aligned}
& R_{1}=\sqrt{\left(\gamma-\epsilon_{2}\right)^{2}\left(1-\cos \theta_{\mathrm{sc}}\right)^{2}+1-\cos ^{2} \theta_{\mathrm{sc}}}, \\
& R_{2}=\sqrt{\left(\gamma+\epsilon_{1}\right)^{2}\left(1-\cos \theta_{\mathrm{sc}}\right)^{2}+1-\cos ^{2} \theta_{\mathrm{sc}}}
\end{aligned}
$$


A kinematic limit for $\gamma$ is imposed for any given $\theta_{\mathrm{sc}}, \epsilon_{2}$, and $\epsilon_{1}$. In the Thomson approximation (i.e., $\gamma \epsilon_{1} \ll m_{e} c^{2}$ ), the expression is (Brunetti, 2000, Equation (34)):

$$
\gamma_{\min }=\sqrt{1+\frac{\left(\epsilon_{2}-\epsilon_{1}\right)^{2}}{2 \epsilon_{1} \epsilon_{2}\left(1-\cos \theta_{s c}\right)}} .
$$

This kinematic constraint is equivalent to Equation (5.7) in the highly relativistic regime.

Figure 5.4 shows normalized up-scattered photon spectra for an incident photon scattered by an isotropic electron distribution for different scattering angles $\theta_{\text {sc }}$ calculated using Equation (5.13). We also show the spectrum resulting from an isotropic photon distribution, based on the same equation (dotted line), and from Jones' approximate formula (Equation (5.2), dashed line). In the ultrarelativistic regime shown in panel (a), the results are similar to those shown in Figure 2 of Moskalenko \& Strong (2000). Note, however, that these authors plot the normalized emissivity as a function of $\epsilon_{2} / \epsilon_{2}^{\max }$ instead of $\epsilon_{2} / \epsilon_{1}$, as we do here. As $\gamma$ decreases from 100, to 10, to 5 in panels $(\mathrm{a})-(\mathrm{c})$, the spectra at different scattering angles are all peaked at the highest possible energies of the upscattered photons, with the maximum deviations in emissivity from the isotropic case occurring for $\theta_{\mathrm{sc}} \approx \pi$. However, as the electrons enter the mildly relativistic regime $(\gamma=10,5$, and 2 in panels $(b)-(d)$, respectively), collisions with smaller scattering angles $\theta_{\mathrm{sc}}$ contribute significantly to the emissivity at small $\epsilon_{2} / \epsilon_{1}$. It is interesting to note that Jones' approximate formula (dashed lines) describes the isotropic case quite accurately for $\gamma \gtrsim 5$, in comparison with the exact calculations (dotted lines). Only at small values of $\gamma$ (panel $(d)$, for which $\gamma=2$ ) does the spectrum from Jones' approximate formula deviate significantly from the exact calculation for an isotropic photon distribution, both in the spectral shape and the upper cutoff energy. 

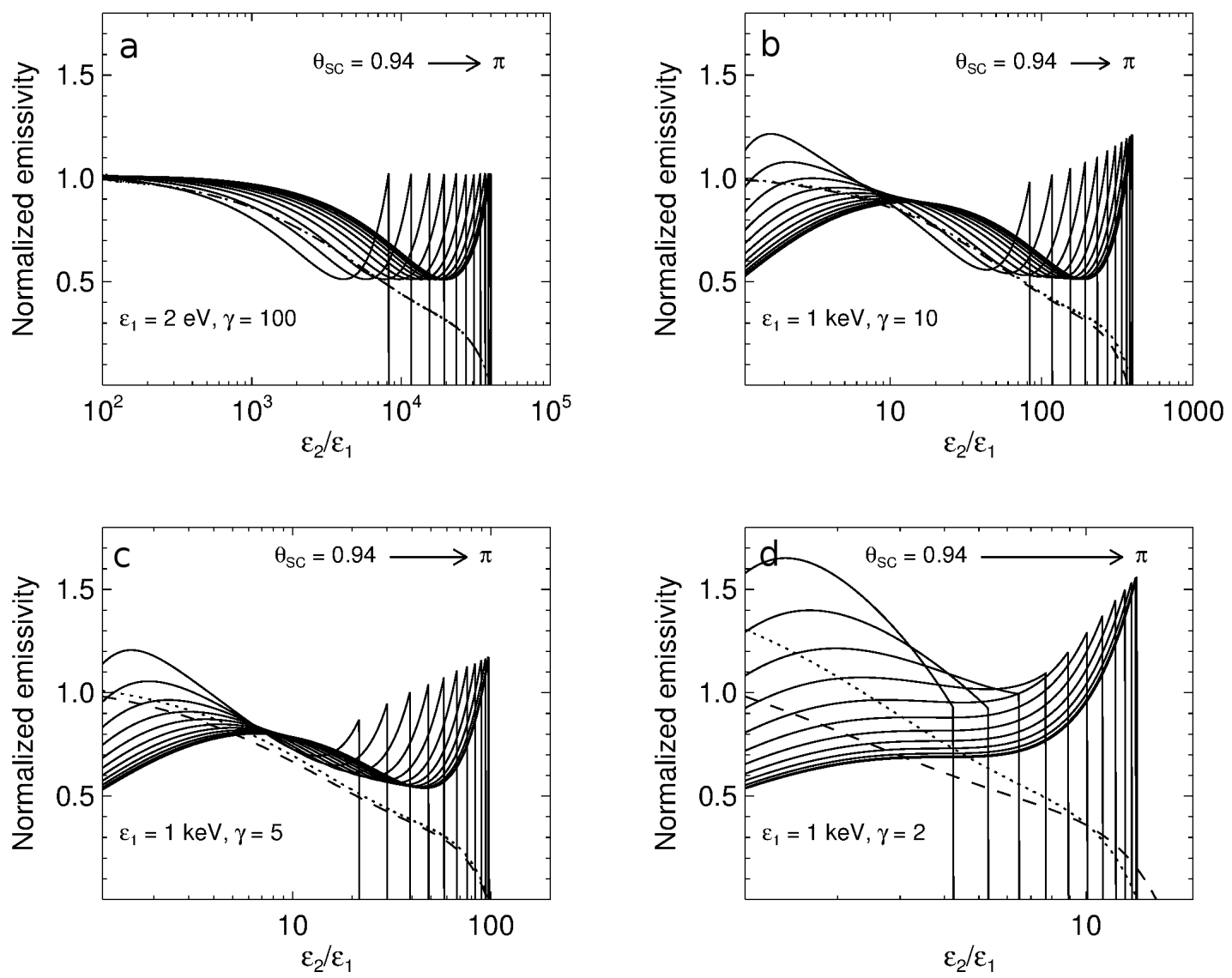

Fig. 5.4. - Upscattered photon spectra from incident photons scattered by a monoenergetic isotropic electron distribution at different scattering angles $\theta_{\mathrm{sc}}$ (solid lines) using the exact ICS calculation. The dotted and dashed lines are the results for an isotropic photon distribution by averaging over all the scattering angles, based on the exact formula (Equation (5.13)) and the Jones' formula (Equation (5.2)) respectively. Panel (a) is for the scattering between the incident photons with $\epsilon_{1}=2 \mathrm{eV}$ and electrons with $\gamma=100$, i.e., in the ultrarelativistic regime. The other panels (b)-(d) are for the mildly relativistic case, with the incident photon energy $\epsilon_{1}=1 \mathrm{keV}$ and electrons with $\gamma=2,5$, and 10 .

Adopting the same geometry as shown in Figure 5.1, the resulting ICS flux can be obtained by integrating $d^{2} R\left(\Omega_{\gamma}, \epsilon_{1}\right) / d \epsilon_{2} d \Omega_{\gamma}$ over the solid angle $\Omega_{\gamma}$ of any incident photon distribution $Q_{\gamma}(\theta, \phi)$, i.e., 


$$
\frac{d R(\lambda)}{d \epsilon_{2}}=\int_{0}^{2 \pi} d \phi \int_{0}^{\pi} \sin \theta d \theta Q_{\gamma}(\theta, \phi) \frac{d^{2} R\left(\Omega_{\gamma}\right)}{d \epsilon_{2} d \Omega_{\gamma}}
$$

Figure 5.5 shows upscattered photon spectra resulting from an isotropic distribution (i.e., $\left.Q_{\gamma}=1 /(4 \pi)\right)$ of monoenergetic photons interacting with an isotropic distribution of electrons. The incoming photon energy is assumed to be $\epsilon_{1}=0.2 \mathrm{keV}$ (left panel) and $\epsilon_{1}=2 \mathrm{keV}$ (right panel) with a photon density of $10^{7} \mathrm{~cm}^{-3}$. The electron kinetic energy has a power-law form $\sim(\gamma-1)^{-\delta}$ with $\delta=3$. The lower and upper limits are assumed to be $\gamma_{1}=1.02(10 \mathrm{keV}), \gamma_{2}=30(\approx 14.8 \mathrm{MeV}$, left panel $)$, and $\gamma_{2}=10(\approx 4.6 \mathrm{MeV}$, right panel). The results are normalized to one electron above $\sim 0.5 \mathrm{MeV}$ as was done previously (Section 5.2.1).

The main difference between the ultrarelativistic case and the mildly relativistic case is that spectra in the latter regime are significantly steeper than the classic relation $\alpha \sim(\delta+1) / 2$. Jones' approximate formula (Equation (5.2)) describes the upscattered photon spectra quite well (the thin-dashed curves) at most photon energies $\epsilon_{2}$, the largest deviations occurring at the lowest ratios of $\epsilon_{2} / \epsilon_{1}$.

Our results for ICS of an anisotropic photon field on an isotropic distribution of mildly relativistic electrons, not shown here, are qualitatively similar to those reported in Section 5.2.1 for the ultrarelativistic case (Figure 5.3). The main difference in this case is that while the emissivity increases with increasing values of $\lambda$, the variation from $\lambda=0$ to $\lambda=\pi / 2$ is somewhat less than is seen for the ultra-relativistic case for $\epsilon_{2} / \epsilon_{1} \gtrsim 10-20$. For smaller values of $\epsilon_{2} / \epsilon_{1}$, the spectra become relatively insensitive to $\lambda$. This is not unexpected because at lower electron energies the emission pattern becomes quite broad and the directionality of the source therefore decreases. 

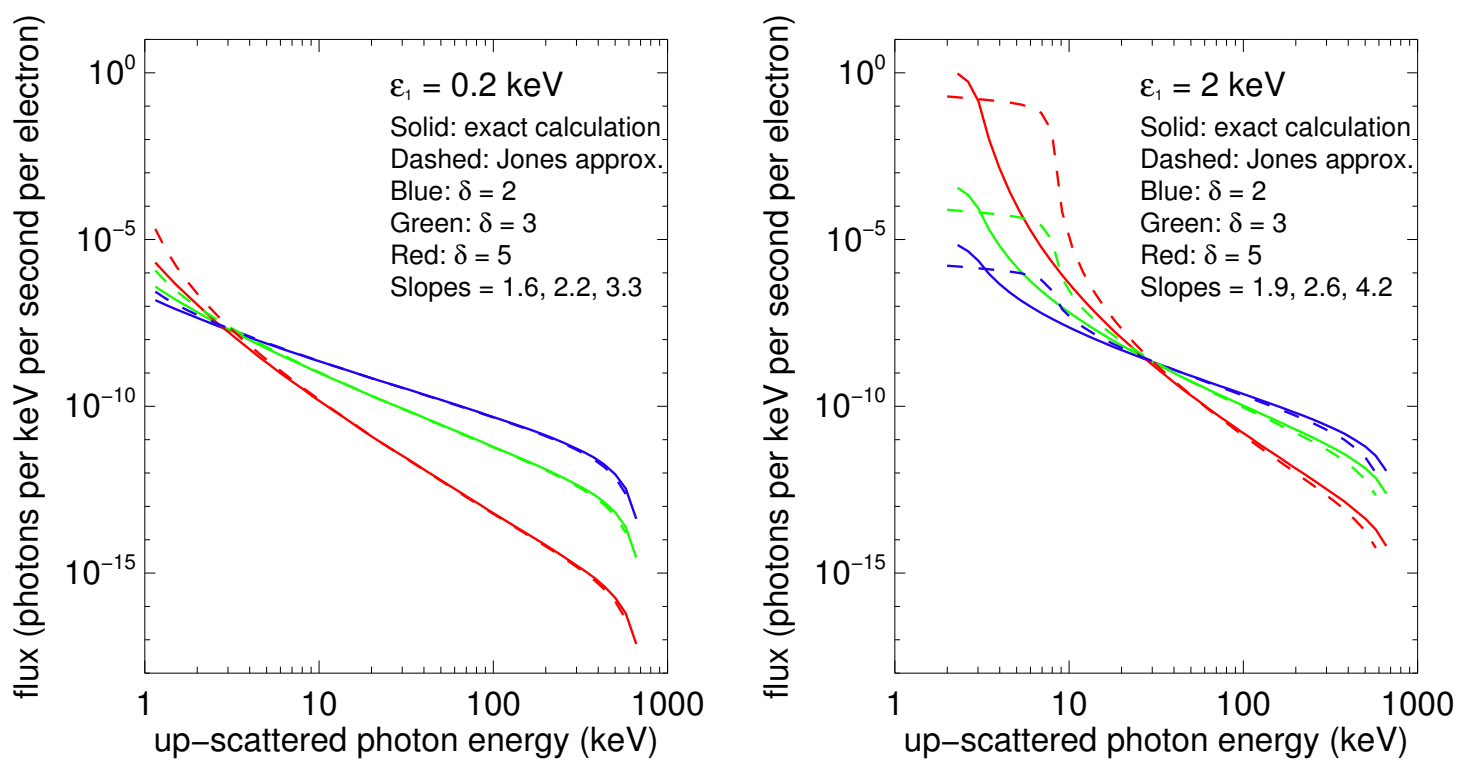

Fig. 5.5.- Upscattered photon spectra at the Sun (photons per keV per second per electron) for the electron energy distribution with different spectral indices $-\delta=2$ (blue), 3 (green), and 5 (red). The dashed line is the result from Jones' approximate formula (Equation (5.2)) for the purpose of comparison. The incident photon energy is respectively $0.2 \mathrm{keV}$ and $2 \mathrm{keV}$ in the left and right panels. The incident photon density is assumed to be $10^{7} \mathrm{~cm}^{-3}$. The lower and upper limits to the electron energy spectrum are $\gamma_{1}=1.02(\approx 10 \mathrm{keV}$, both panels $), \gamma_{2}=30(\approx 14.8 \mathrm{MeV}$, left panel $)$, and $10(\approx 4.6 \mathrm{MeV}$, right panel). The slopes are obtained by fitting a power law between $20 \mathrm{keV}$ and $80 \mathrm{keV}$.

\subsubsection{ICS of Isotropic Photons on Anisotropic Electron Dis- tributions}

Anisotropic electron distributions may arise during solar flares as a result of acceleration, injection, and/or transport effects. Two idealized cases are commonly considered: electrons in a beam or electrons in a plane. We refer to the latter type as a "pancake" distribution. In the presence of a magnetic field, beamed electrons are typically those with pitch angles $\alpha_{p}$ such that $u=\cos \alpha_{p}$ is confined to be near 1 or -1 (i.e., they stream along the magnetic field) and pancake distributions are those where 
$u \sim 0$ (i.e., electrons are largely perpendicular to the magnetic field). We consider both of these distributions in this section, and calculate the ICS spectra resulting from their interaction with an isotropic photon field. We use an isotropic photon field for computational ease but note that the effect of an anisotropic photon field manifests itself largely as a geometrical effect resulting in a correction factor of order $4 \pi / \Omega_{\gamma}$ (Moskalenko \& Strong, 2000). For ICS of photospheric photons scattering on ultrarelativistic regime, the effect is therefore a factor of about two.

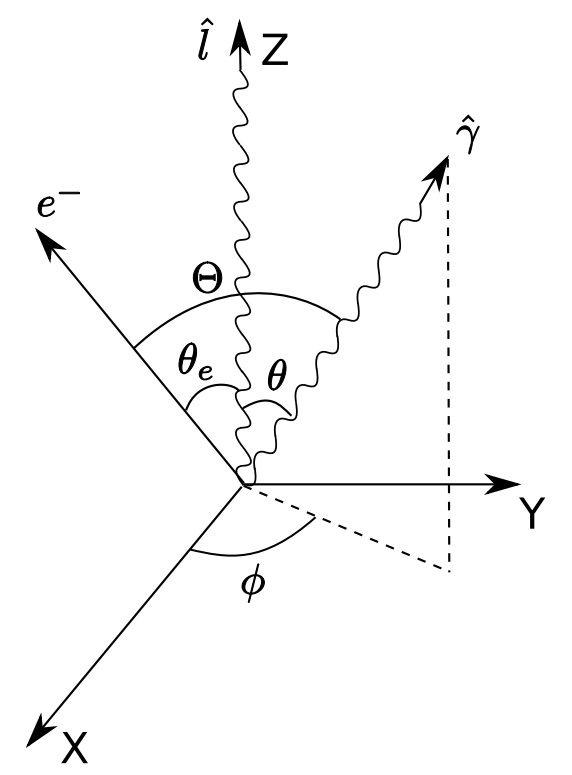

Fig. 5.6. - Geometry and angles used to calculate the ICS emission between a unidirectional photon beam and a unidirectional electron beam, each normalized to unit density. The $Z$-axis is along the direction of the up-scattered photons (i.e., the LOS $\hat{l}) ; \hat{\gamma}$ is the unit vector along the incident photon beam, with $\theta$ and $\phi$ as the polar and azimuthal angles; the direction of the electron is placed in the $X-Z$ plane, with an angle $\theta_{e}$ away from the LOS; $\Theta$ is the angle between the electron and the incident photon.

Brunetti (2000) derived the ICS emissivity from unidirectional, mono-energetic photons interacting with a unidirectional electron distribution with an arbitrary energy distribution. We adopt these results here but transform to a more convenient 
geometry, as shown in Figure 5.6. In our geometry, the LOS is along the $Z$-axis which is taken to be the direction of the upscattered photon ( $\hat{l}$ is the unit vector). The incident photons have a direction of $\Omega_{\gamma}(\theta, \phi)$, where $\theta$ and $\phi$ are the polar and azimuthal angles respectively. The direction of the electron momenta is taken to be in the $X-Z$ plane, with an angle $\theta_{e}$ away from the LOS. $\Theta$ is the angle between the electron and the incident photon. The derived emission rate is then (adapted from Equation (26) of Brunetti 2000)

$$
\begin{aligned}
\frac{d^{3} R\left(\Omega_{\gamma}, \theta_{e}\right)}{d \epsilon_{2} d \Omega_{\gamma} d \Omega_{e}}= & 2 \pi r_{0}^{2} \frac{\epsilon_{2}}{\epsilon_{1}} \frac{(\cos \theta-1)^{2}}{\epsilon_{2}-\epsilon_{1}}\left(\gamma_{0}-\frac{1}{\gamma_{0}}\right)\left\{\frac{1}{(\cos \theta-1)^{2}}\right. \\
& \left.+\left[\frac{1}{\cos \theta-1}+\frac{\left(\epsilon_{2}-\epsilon_{1}\right)^{2}}{\epsilon_{1} \epsilon_{2}\left(\cos \theta_{e}-\cos \Theta\right)^{2}} \frac{1}{\gamma_{0}^{2}}\right]^{2}\right\} K_{e} F_{e}\left(\gamma_{0}\right) Q_{e}\left(\Omega_{e}\right) Q_{\gamma}\left(\Omega_{\gamma}\right)
\end{aligned}
$$

where the Lorentz factor $\gamma_{0}=1 / \sqrt{1-\beta_{0}^{2}}$ of the electron is determined by kinematics

$$
\beta_{0}=\frac{\epsilon_{2}-\epsilon_{1}}{\epsilon_{2} \cos \theta_{e}-\epsilon_{1} \cos \Theta}
$$

$K_{e} F_{e}\left(\gamma_{0}\right) Q_{e}\left(\Omega_{e}\right)$ is the differential electron number density at $\gamma_{0}$, and $\Theta$ is related to $\theta, \phi$, and $\theta_{e}$ by geometry

$$
\cos \Theta=\cos \theta \cos \theta_{e}+\sin \theta \sin \theta_{e} \cos \phi
$$

For an isotropic incident photon field $Q_{\gamma}(\theta, \phi)=1 /(4 \pi)$, integration over the solid angle $\Omega_{\gamma}$ yields the ICS rate $d^{2} R\left(\theta_{e}\right) / d \epsilon_{2} d \Omega_{e}$ for a given direction of the electron $\Omega_{e}$.

Figure 5.7 shows the normalized ICS emission rates as a function of the source electron angle $\theta_{e}$ at different ratios of $\epsilon_{2} / \epsilon_{1}$. As expected, the emission is most favorable when the electron direction is along the LOS (i.e., $\theta_{e}=0$ ), and drops rapidly 
with increasing $\theta_{e}$. In addition, the emission cone opens up gradually from the ultrarelativistic case (large $\epsilon_{2} / \epsilon_{1}$ ) to the mildly relativistic case (smaller $\epsilon_{2} / \epsilon_{1}$ ). The width of the emission cone is $\sim 1 / \gamma_{\min }$, where $\gamma_{\min } \approx \frac{1}{2} \sqrt{\epsilon_{2} / \epsilon_{1}}$ is the minimum electron Lorentz factor required to up-scatter $\epsilon_{1}$ to $\epsilon_{2}$.

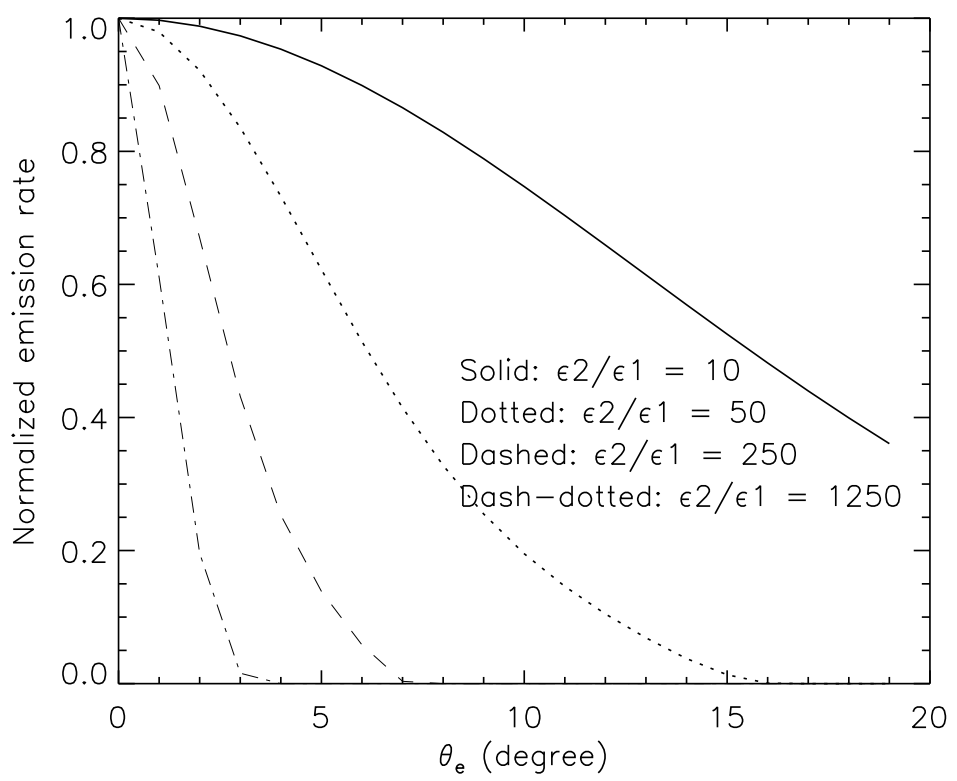

Fig. 5.7.- Normalized ICS emission rate at different angles of the electron relative to the $\operatorname{LOS}\left(\theta_{e}\right)$. The solid, dotted, dashed, and dash-dotted curves are for $\epsilon_{2} / \epsilon_{1}=$ $10,50,250$, and 1250, respectively.

The ICS emissivity can be obtained by integrating over the solid angle $\Omega_{e}$ for any given electron distribution $Q_{e}\left(\Omega_{e}\right)$

$$
\frac{d R}{d \epsilon_{2}}=\int d \Omega_{e} \frac{d^{2} R\left(\theta_{e}\right)}{d \epsilon_{2} d \Omega_{e}} K_{e} F_{e}\left(\gamma_{0}\right) Q_{e}\left(\Omega_{e}\right)
$$

The most favorable and extreme case is for a monodirectional electron beam along the LOS. In the ultrarelativistic regime, the ICS emission rates (Figure 5.8, dashed curves) are four to five orders of magnitude higher than the isotropic case from 10-100 

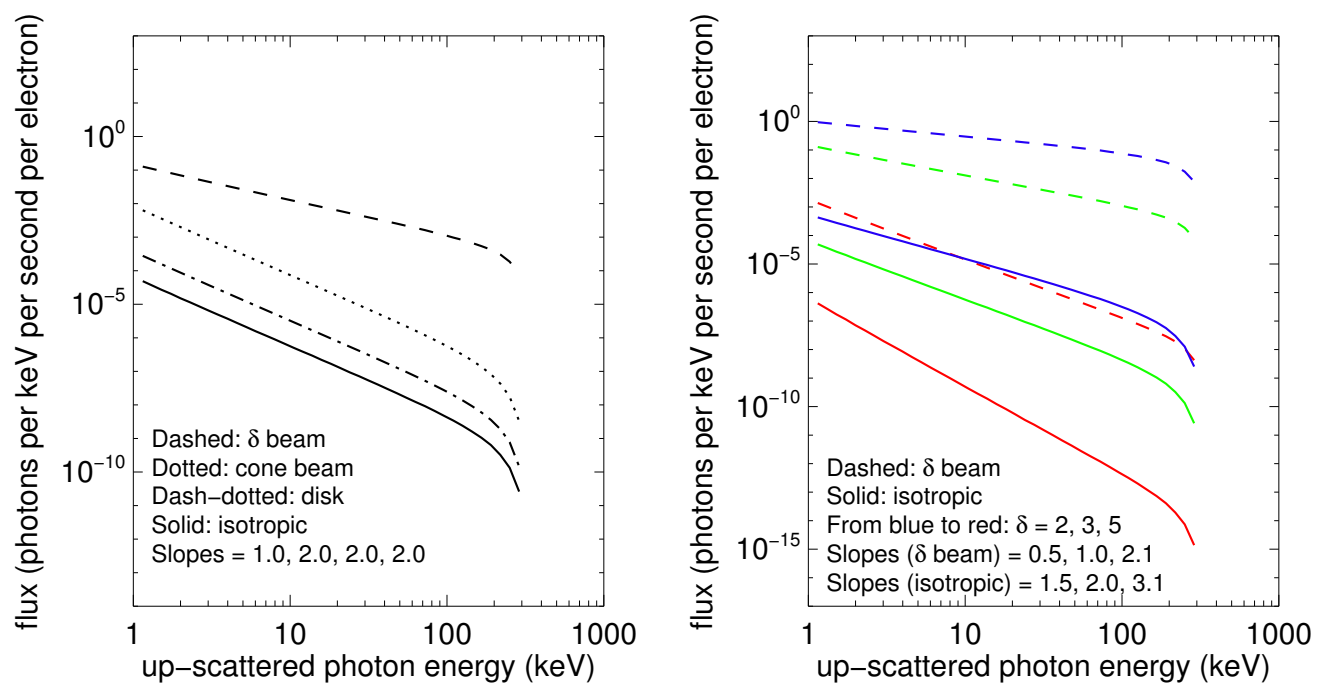

Fig. 5.8.- Upscattered photon spectra at the Sun (photons per keV per second per electron) from anisotropic electron distributions along the LOS in the ultrarelativistic regime. Left: comparison between the spectra from a monodirectional electron beam (dashed line), a cone-like distribution of electrons with a half width $\Delta \theta_{b}=10^{\circ}$ (dotted line), a pancake electron distribution with a half-angle width of $\Delta \theta_{p}=10^{\circ}$ (dashdotted line), and an isotropic electron distribution (solid line). The electron energy spectral index is $\delta=3$. Right: the ICS spectra resulting from the $\delta$-function beam (dashed) and isotropic (solid) distributions with different electron spectral indices $\delta$. The blue, green, and red curves are for $\delta=2,3$, and 5 , respectively. All of the curves assume an incident photon energy of $2 \mathrm{eV}$, and the number density is assumed to be $10^{12} \mathrm{~cm}^{-3}$.

keV (Figure 5.8, solid curves). The spectra are significantly flatter, with a photon spectral index, $\alpha \sim(\delta-1) / 2$, similar to the analytical results in Brunetti $(2000$, see his Equation (28); note that his result is expressed in terms of an energy emissivity rather than a photon emissivity). In the mildly relativistic regime (Figure 5.9, dashed curves), the ICS photon spectrum is likewise significantly flatter than the isotropic case, although it is somewhat steeper than the ultrarelativistic case. Here, too, the ICS emission is enhanced over the isotropic case: by $2.5-3.5$ orders of magnitude for EUV photons and by 1-2.5 orders of magnitude for SXR photons. 

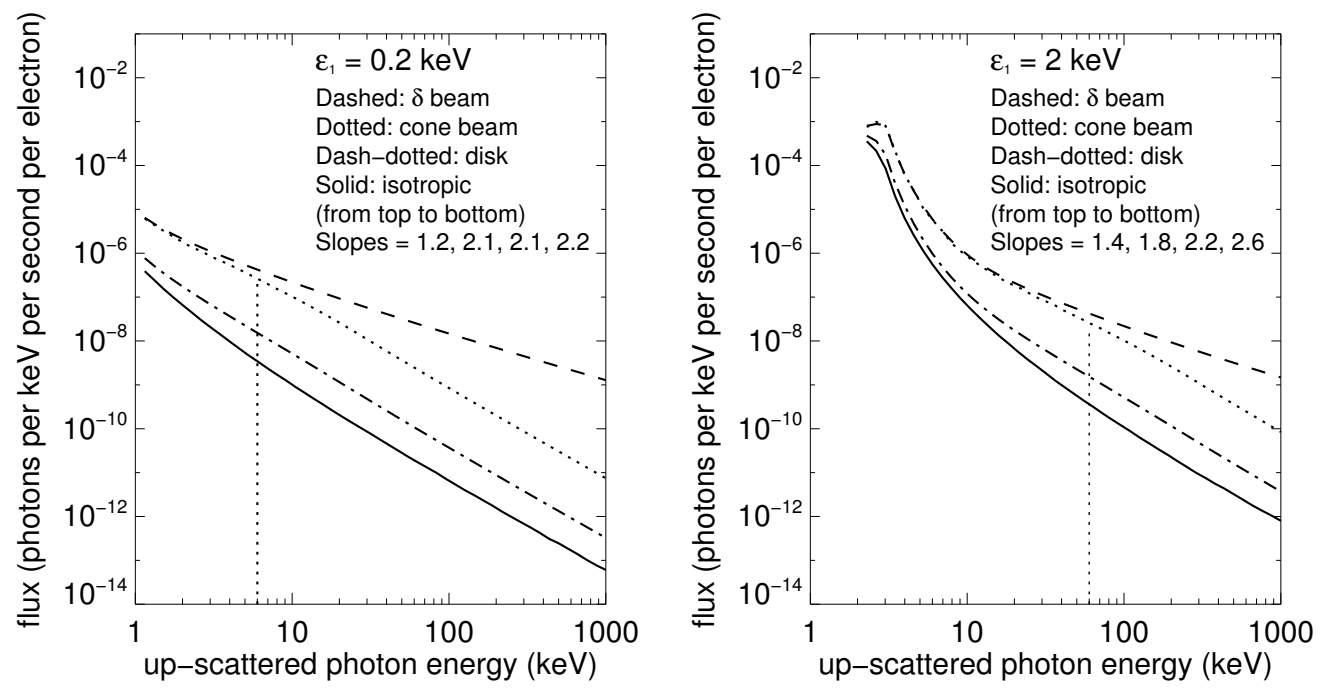

Fig. 5.9.- Upscattered photon spectra at the Sun (photons per keV per second per electron) from anisotropic electron distributions along the LOS in the mildly relativistic regime. Left: comparison between the spectra from different types of electron angular distributions. The dashed, dotted, dashed-dotted, and solid curves are, respectively, for a monodirectional beam, a cone beam (with a half-width $\Delta \theta_{b}=$ $10^{\circ}$ ), a pancake electron distribution (with a half-angle thickness of $\Delta \theta_{p}=10^{\circ}$ ), and an isotropic electron field. The incident photons have an energy of $0.2 \mathrm{keV}$ with a number density of $10^{7} \mathrm{~cm}^{-3}$, and the electron energy spectral index is $\delta=3$. Right: all parameters are the same except that the incident photon energy is $2 \mathrm{keV}$. The spectral slopes are obtained by fitting the spectra between 20 and $80 \mathrm{keV}$. The vertical dotted lines indicate the location of $\left(\epsilon_{2} / \epsilon_{1}\right)_{0}=30$ that corresponds to the transition of the broken power law in the spectra of the cone-beam distribution.

A more physically realistic beamed electron distribution is not mono-directional, but has a finite angular width. We therefore consider electrons confined to a cone with an angular half-width of $\Delta \theta_{b}$ with its axis along the LOS:

$$
Q_{e}\left(\Omega_{e}\right)=\frac{H\left(\Delta \theta_{b}-\theta_{e}\right)}{2 \pi\left(1-\cos \Delta \theta_{b}\right)}
$$

Results are again calculated in both the ultra-relativistic (Figure 5.8, dotted curve) and mildly relativistic regimes (Figure 5.9, dotted curves). For the ultrarelativistic 
case, the resulting spectra are steeper than that resulting from the $\delta$-function beam and, in fact, have the same shape as the isotropic case, but differ in magnitude by a constant factor. The reason is that since the width of the electron emission cone goes as $1 / \gamma$, electrons with smaller $\gamma$ have broader emission cones than those with larger $\gamma$. Lower energy electrons therefore contribute to an increasing degree as $\theta$ deviates from the LOS, resulting in a steeper spectrum. In fact, there should be no significant difference from the isotropic case except for a geometric filling factor such that the emissivity is enhanced by a factor $\sim 4 / \Delta \theta_{b}^{2}$ for small values of $\Delta \theta_{b}$.

The ICS spectra in the mildly relativistic regime present more complex features (Figure 5.9): they are similar to the spectra of the monodirectional beam case at small values of $\epsilon_{2} / \epsilon_{1}$, and transition to a form similar to that obtained for the ultrarelativistic case at large values of $\epsilon_{2} / \epsilon_{1}$. The spectra resemble a broken power law that is harder at small $\epsilon_{2} / \epsilon_{1}$ and softer at large $\epsilon_{2} / \epsilon_{1}$. This can also be understood by the variation of the emission cone with $\epsilon_{2} / \epsilon_{1}$-when $\epsilon_{2} / \epsilon_{1}$ is small, the emission cone is much wider than the beamwidth $\Delta \theta_{b}$. That means all the electrons in the cone beam contribute near equally to the ICS flux, so the spectra are similar to those of the $\delta$-function beam case. As $\epsilon_{2} / \epsilon_{1}$ gets larger, the width of the emission cone decreases and the emission asymptotically approaches the ultrarelativistic regime. The transition happens when the half-width of the emission cone $1 /\left(2 \gamma_{\min }\right) \approx \sqrt{\epsilon_{1} / \epsilon_{2}}$ reaches the half-width of the cone beam $\Delta \theta_{b}$, which sets a critical value of $\left(\epsilon_{2} / \epsilon_{1}\right)_{0} \approx 1 / \Delta \theta_{b}^{2}$ for the transition. In Figure 5.9, where $\Delta \theta_{b}=10^{\circ}$, the transition occurs where $\left(\epsilon_{2} / \epsilon_{1}\right)_{0} \approx 30$, marked by the vertical dotted lines in Figure 5.9.

We now consider a pancake electron anisotropy. In the case of a magnetized plasma, such a distribution arises when the electron pitch angles are largely perpendicular to the magnetic field $(u \sim 0)$. Figure 5.10 describes the geometry for 
calculating the ICS emission with a pancake electron angular distribution: the electrons are taken to be confined near the $X-Y$ plane, with the LOS along the $X$-axis. The direction of the electron $\Omega_{e}$ is described by the polar and azimuthal angles $\theta^{\prime}$ and $\phi^{\prime}$. The electrons are confined in a polar angles from $\pi / 2-\Delta \theta_{p}$ to $\pi / 2+\Delta \theta_{p}$, where $\Delta \theta_{p}$ is the half-thickness of the disk. The electron angular distribution $Q_{e}\left(\Omega_{e}\right)$ is assumed to be

$$
Q_{e}\left(\Omega_{e}\right)=\frac{H\left(\Delta \theta_{p}-\left|\theta^{\prime}-\pi / 2\right|\right)}{4 \pi \sin \Delta \theta_{p}},
$$

where we again have $\int Q_{e}\left(\Omega_{e}\right) d \Omega_{e}=1$. The angle between the electron and the LOS $\theta_{e}$ is related to the polar and azimuthal angles of the electron direction $\left(\theta^{\prime}, \phi^{\prime}\right)$ by

$$
\cos \theta_{e}=\cos \phi^{\prime} \sin \theta^{\prime}
$$

Hence, the ICS emissivity can be obtained by integrating $d^{2} R\left(\theta_{e}\right) / d \epsilon_{2} d \Omega_{e}$ over the solid angle $\Omega_{e}$ in Equation (5.20).

The results for both the ultra- and mildly relativistic cases are shown in Figures 5.8 and 5.9 as dash-dotted curves, using the same parameters as those in the monodirectional and cone-beam cases, with the half-thickness of the disk $\Delta \theta_{p}=10^{\circ}$. The spectra are similar to those of the cone beam, except that the magnitude is now enhanced by a factor of $\sim 1 / \Delta \theta_{p}$ for small $\Delta \theta_{p}$ - e.g., about a factor of six when $\Delta \theta_{p}=10^{\circ}$ - compared to the fully isotropic case. There is less obvious complexity to the photon spectrum resulting from a pancake electron distribution. The reasons is that in the plane of the pancake, there is no cutoff effect with increasing $\epsilon_{2} / \epsilon_{1}$; it is only present in the polar angle, perpendicular to the pancake. Since, in the mildly relativistic case, the photon spectrum steepens for smaller values of $\epsilon_{2} / \epsilon_{1}$, the effect manifests itself subtly in Figure 5.9 as a convergence between the dotted curves (cone-beam anisotropy) and the solid line curves (isotropic case) for $\epsilon_{2} / \epsilon_{1}<\left(\epsilon_{2} / \epsilon_{1}\right)_{0}$, 


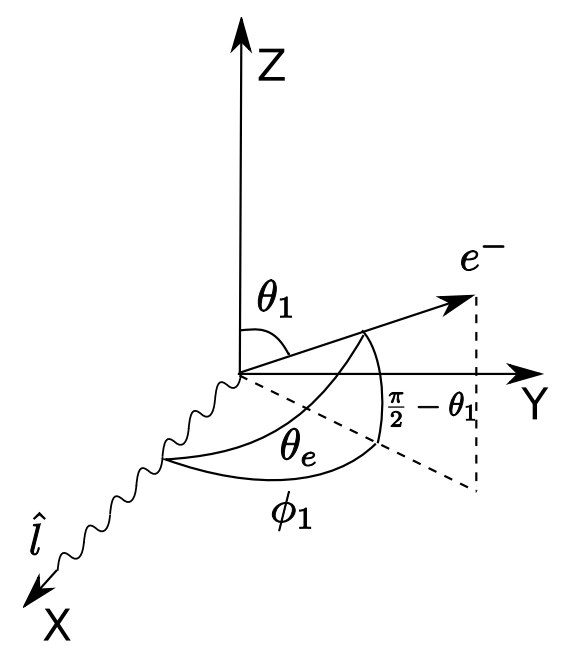

Fig. 5.10. - Geometry and angles used to calculate the ICS emission between a disklike electron angular distribution and an isotropic photon field. The disk is in the $X-Y$ plane. The $X$-axis is along the LOS $\hat{l}$. $\theta^{\prime}$ and $\phi^{\prime}$ are the polar and azimuthal angles of the electron direction. $\theta_{e}$ is the angle between the electron and LOS. The electrons are confined in polar angles from $\pi / 2-\Delta \theta_{p}$ to $\pi / 2+\Delta \theta_{p}$, where $\Delta \theta_{p}$ is the half-angular-thickness of the disk.

i.e., the photon spectrum does not steepen as much as it otherwise would.

In summary, ICS on anisotropic electron distributions can be far more effective than it is on an isotropic electron distribution. The reason is that ICS is highly directional - those electrons that are far from the LOS do not contribute to ICS. As a result, all other things being equal, an anisotropic electron distribution results in significantly enhanced ICS emission for favorable viewing geometries, i.e., when the electron momenta are near the LOS. 


\subsection{Thin-target Non-thermal Bremsstrahlung Emis- sion}

In this section, we briefly review results from thin-target non-thermal bremsstrahlung emission and compare this mechanism with ICS. As noted in the Section 5.1, the coronal HXR sources have been interpreted in terms of non-thermal bremsstrahlung emission in general. Since the plasma density in the corona is low, the "thin-target" regime is usually assumed. There are exceptions, of course: collisionally thick coronal HXR sources may occur as a result of a high ambient plasma density (Veronig \& Brown, 2004) or trapping (e.g., Krucker et al., 2007b). We only consider the thintarget regime here.

The total bremsstrahlung cross-section is composed of two parts: that due to collisions between incident electrons and target ions, and that due to collisions between incident electrons and target electrons. The contribution of EEB to the total bremsstrahlung emission is negligible at non-relativistic electron energies. For this reason it is typically ignored when considering HXR photon energies less than a few $\times 100 \mathrm{keV}$, and the emission is assumed to be the result of EIB alone. However, as emphasized by Kontar et al. (2007), the neglect of EEB is not necessarily justified when considering photon energies $\gtrsim 300 \mathrm{keV}$. For an isotropic distribution of nonrelativistic electrons with a power-law density distribution and a spectral index $\delta$, the HXR photon spectral index due to thin-target EIB is $\alpha \sim \delta+1 / 2$. For incident electrons approaching relativistic energies, the thin-target EIB spectrum flattens and $\alpha \sim \delta$. The EEB contribution has a photon spectral index $\alpha \sim \delta-1 / 2$ at lower energies and flattens somewhat (by 0.2-0.3) at higher energies. Hence, the EEB contribution can further flatten the total photon spectrum as photon energies exceed a 
few hundred keV. Previous work (e.g., Haug, 1975b) has shown, however, that for isotropic distributions of incident electrons on a pure hydrogen plasma, the contribution of EEB is significantly less than EIB. For example, EEB contributes roughly $27 \%, 18 \%$ and $12.5 \%$ of the total photon emissivity at $400 \mathrm{keV}$ for emission from a power-law distribution of energetic electrons with $\delta=3,4$, and 5 , respectively. The EEB contribution results in a corresponding hardening of the photon spectral index in the amount of $0.2,0.15$, and 0.1 , respectively, for photon energies $>200 \mathrm{keV}$.

In Chapter 1.2.4, Equation 1.16 gives the X-ray flux due to thin-target EIB bremsstrahlung radiation from an isotropic electron distribution in a homogeneous source. Here we expand it into a more general case: thin-target EIB bremsstrahlung radiation that results from an anisotropic electron distribution. The X-ray photon emission rate (photons $\mathrm{cm}^{-3} \mathrm{~s}^{-1} \mathrm{keV}^{-1}$ ) can be described as

$$
\begin{aligned}
\frac{d R}{d \epsilon_{2}} & =n_{i} \int_{\Omega_{e}} \int_{\gamma_{\min }}^{\infty} f_{e}(\gamma) v_{e}(\gamma) \sigma\left(\epsilon_{2}, \gamma, \theta_{e}\right) d \gamma d \Omega_{e} \\
& =n_{i} \int_{\gamma_{\min }}^{\infty} d \gamma v_{e}(\gamma) K_{e} F_{e}(\gamma) \int_{\Omega_{e}} d \Omega_{e} \sigma\left(\epsilon_{2}, \gamma, \theta_{e}\right) Q_{e}\left(\Omega_{e}\right),
\end{aligned}
$$

where $f_{e}\left(\gamma, \Omega_{e}\right)=K_{e} F_{e}(\gamma) Q_{e}\left(\Omega_{e}\right)$ is the differential electron number density as a function of the electron energy and solid angle $\Omega_{e}$, in which the electron angular distribution is $Q_{e}\left(\Omega_{e}\right) . \sigma\left(\epsilon_{2}, \gamma, \theta_{e}\right)$ is the bremsstrahlung cross-section differential in the photon energy $\epsilon_{2}$, electron energy $\gamma$, and $\theta_{e}$, the angle between the incoming electron direction and the emitted photon direction (or LOS). The doubly differential bremsstrahlung cross-section consists of both the EIB component $\sigma_{\text {ei }}\left(\epsilon_{2}, \gamma, \theta_{e}\right)$ given by Koch \& Motz (1959, Equation (2BN)) and the EEB component $\sigma_{\mathrm{ee}}\left(\epsilon_{2}, \gamma, \theta_{e}\right)$ given by Haug (1975a). Figure 5.11 shows a polar diagram of the normalized thin-target bremsstrahlung emissivity (EIB+EEB) as a function of $\theta_{e}$ and the logarithm of the 
photon energy resulting from a monodirectional ( $\delta$-function) angular distribution and a power-law energy distribution of electrons $(\delta=3)$ extending from $10 \mathrm{keV}$ to 100 $\mathrm{MeV}$. It is compared with ICS from the same distribution of electrons scattering EUV photons $\left(\epsilon_{1}=0.2 \mathrm{keV}\right)$ and $\operatorname{SXR}$ photons $\left(\epsilon_{1}=2 \mathrm{keV}\right)$. The case of photospheric photons $(2 \mathrm{eV})$ scattering from the ultrarelativistic electrons in the distribution is not shown because the emissivity is itself monodirectional. Similar to the ICS emissivity, the bremsstrahlung emissivity peaks in the direction of the incident electrons at large photon energy $\epsilon_{2}$. Its emission pattern is more complex at non-relativistic energies, showing a maximum at $\theta_{e} \sim 30-40^{\circ}$ rather than along the LOS (cf., Massone et al., 2004). In any case, the bremsstrahlung emission pattern is broader than that of ICS as a result of the fact that the energy of a given bremsstrahlung photon is comparable to that of the incident electron $(\gamma \sim \epsilon)$ whereas the electrons responsible for ICS are much more energetic than the upscattered photons considered here $(\gamma \gg \epsilon)$ and their beaming is consequently more pronounced.

For completeness, we have also considered the effect of a low-energy cutoff $E_{c}$ in the electron energy distribution on the spectral index of the photon spectrum for EIB, EEB, and their sum. Our results are consistent with those of Brown et al. (2008) for the case of an isotropic electron distribution and thin-target EIB emission. That is, for photon energies well below the cutoff $\left(\epsilon \ll E_{c}\right)$ the spectral index is very hard $(\alpha \sim 1.5)$. The inclusion of EEB may change the spectral index of the photon spectrum by $\lesssim 0.1$.

We have also calculated the bremsstrahlung photon spectra, for the contributions from both EIB and EEB, resulting from the same electron anisotropies considered in Section 5.2.2, namely, a monodirectional electron beam, a cone-beam distribution with a half-angle width of $\Delta \theta_{b}=10^{\circ}$, and a pancake electron distribution with a 

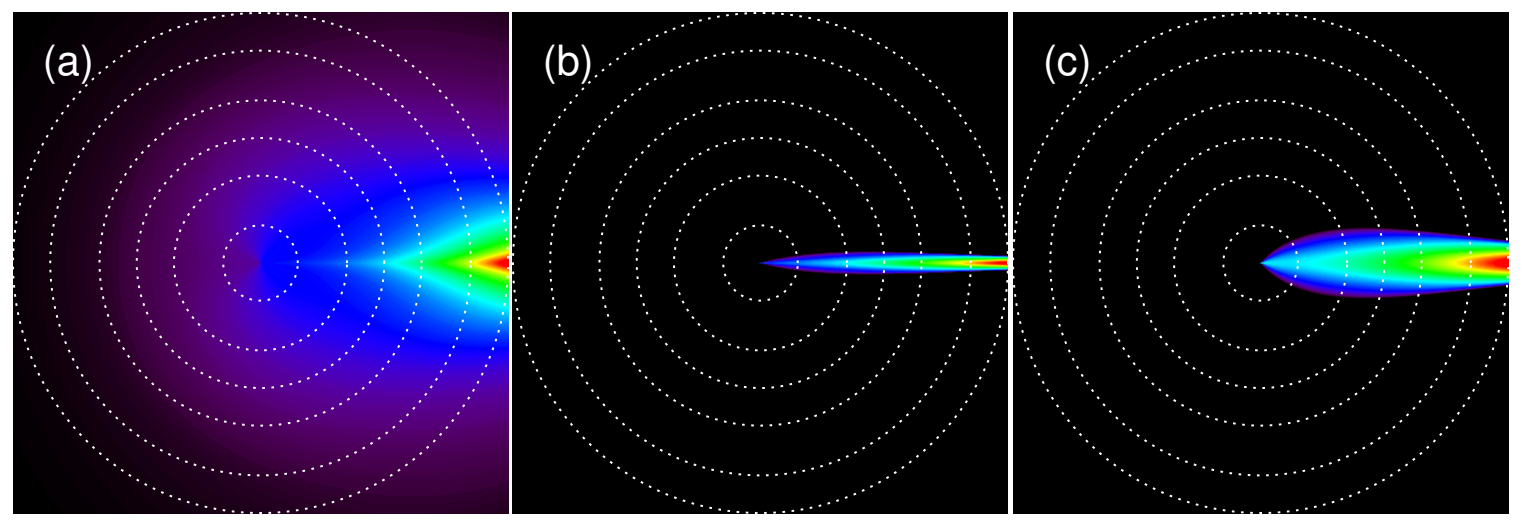

Fig. 5.11.- Polar diagram of the normalized emissivity from a monodirectional electron as a function of the logarithm of the photon energy $\epsilon_{\gamma}$ and the angle of the LOS relative to the beam direction. The electron beam propagates in the $+x$ direction. The electron distribution function is a power law between $10 \mathrm{keV}$ and $100 \mathrm{MeV}$ and has an index $\delta=3$. The emissivity has been multiplied by $\epsilon_{\gamma}^{\delta}$ in all cases. The concentric circles indicate contours of constant photon energy: 20, 50, 100, 200, 500, and $1000 \mathrm{keV}$. (a) Thin-target bremsstrahlung emissivity. The EIB and EEB contributions have been summed; (b) the same for ICS of mono-energetic EUV photons $(0.2 \mathrm{keV})$ up-scattered by the electron beam; (c) same as panel (b), but for SXR photons $(2 \mathrm{keV})$.

half-angle width of $\Delta \theta_{p}=10^{\circ}$. Figure 5.12 shows the corresponding results from a single power-law electron energy distributions with a spectral index $\delta=3.0$ using the same parameters used to compute the examples of ICS emission in the mildly relativistic regime shown in Figure 5.9. The left panel shows the EIB (blue curve) and EEB (red curve) components separately, and the right panel shows the total photon spectra (black curve) including contributions form both EIB and EEB as well as the EIB component alone (blue curve). We note that the resulting spectra are qualitatively similar to those resulting from ICS on these distributions. In particular, the extreme case of a monodirectional electron beam directed along the LOS results in a significant enhancement to the thin-target emissivity and a substantially flatter spectrum than the isotropic case, for which $\alpha=3.5$ for non-relativistic photon energies 

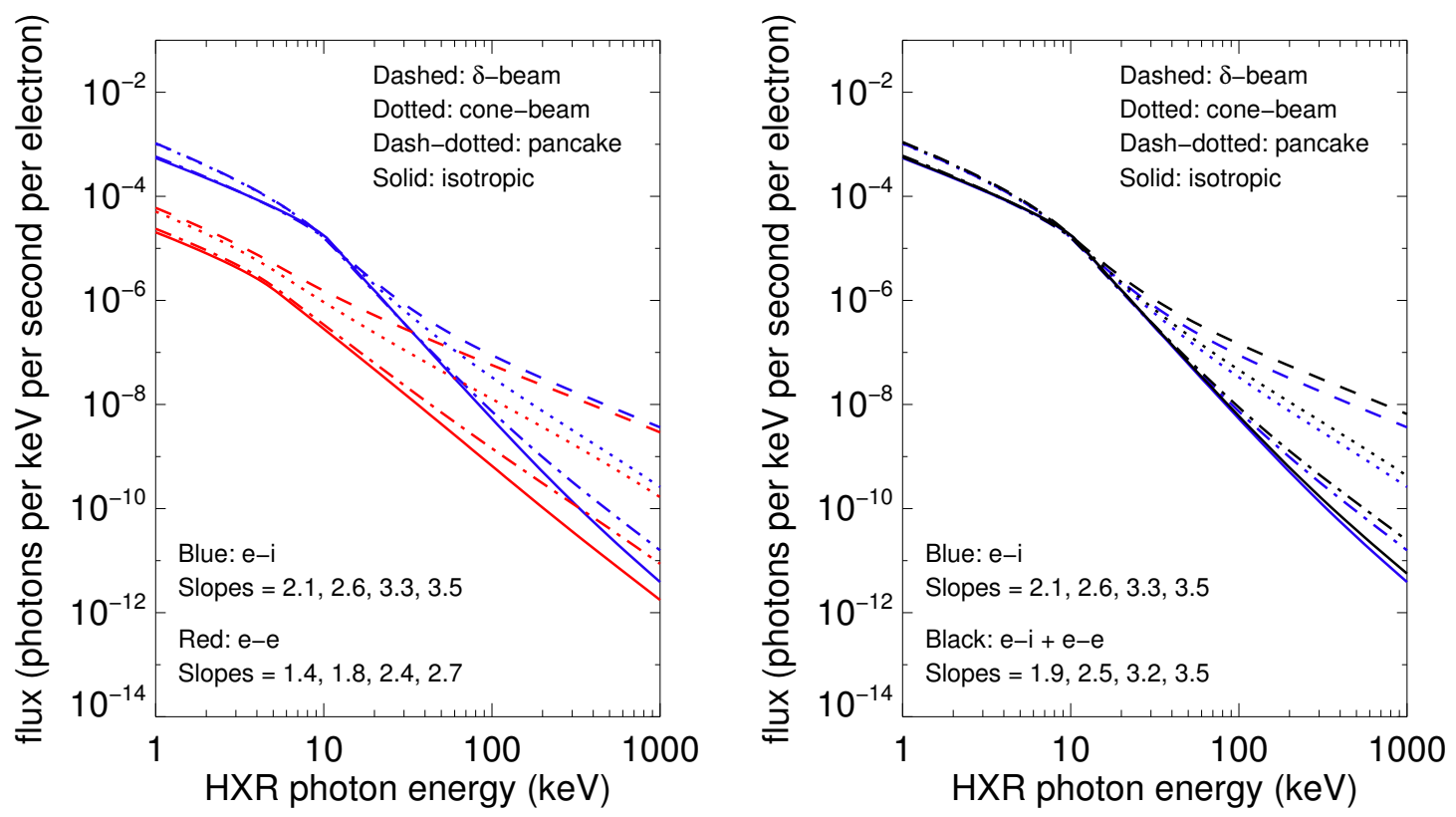

Fig. 5.12.- Bremsstrahlung photon spectra at the Sun (photons per keV per second per electron) from anisotropic electron distributions along the LOS. Different kinds of anisotropies are plotted - a monodirectional electron beam (dashed line), a cone-beam distribution with a half-angle width of $\Delta \theta_{b}=10^{\circ}$ (dotted line), a pancake electron distribution with a half-angle width of $\Delta \theta_{p}=10^{\circ}$ (dash-dotted line), and an isotropic electron distribution (solid line). The electron energy distribution is assumed to have a single power-law form with a spectral index $\delta=3$, extending from $10 \mathrm{keV}$ to 100 $\mathrm{MeV}$ (same as that in Figure 5.9). The results are also normalized to one source electron above $0.5 \mathrm{MeV}$, and the ion number density $n_{i}$ is assumed to be $10^{8} \mathrm{~cm}^{-3}$. The blue and red curves are for the EIB and EEB components of the bremsstrahlung spectra respectively. The black curves are for the total bremsstrahlung spectra. Note that the break in the spectra at $\sim 10 \mathrm{keV}$ is from the lower energy cutoff of the electron distribution at $10 \mathrm{keV}$.

(20-80 keV) and $\alpha=2.9$ for $\gamma$-ray photon energies $(200-800 \mathrm{keV})$. The cone beam and pancake anisotropy result in more modest enhancements and their spectra are intermediate to the isotropic and monodirectional beam (for the cone beam, $\alpha=2.5$ and 2.0 for the $20-80 \mathrm{keV}$ and $200-800 \mathrm{keV}$ ranges, respectively; for the pancake anisotropy, $\alpha=3.2$ and 2.5, respectively). We note that for the monodirectional 
beam, EIB and EEB asymptotically approach equality as the photon energy increases (cf., Dermer \& Ramaty, 1986), but the EEB contribution becomes less prominent in the beam-cone and pancake distributions (see the left panel in Figure 5.12). Note, too, that the degree of enhancement of each of the anisotropic cases relative to the isotropic case is less dramatic than for ICS. There is essentially no enhancement at $10 \mathrm{keV}$ photon energies owing to the fact that the electron distribution cuts off at $10 \mathrm{keV}$, but this changes as the photon energy increases: to an enhancement of perhaps 1.5 orders of magnitude for the mono-directional beam at $100 \mathrm{keV}$; 1 order of magnitude for the cone beam; and a factor of $\sim 2$ for the pancake distribution. This can be understood as a consequence of the more modest degree of directivity of bremsstrahlung emission compared with ICS. The HXR (20-80 keV) and $\gamma$-ray (200-800 keV) spectral indices that result from these cases are summarized in Table 5.1. We conclude from this exercise that the effect of electron anisotropies on mildly relativistic and ultrarelativistic ICS emission is significantly larger than is the case for thin-target bremsstrahlung emission, all other things being equal.

We now turn our attention to the relative roles of ICS and thin-target bremsstrahlung in the production of HXR and continuum $\gamma$-ray emission. As was discussed by MM10, a comparison between the relative roles of ICS and bremsstrahlung is somewhat problematic because the HXR photons resulting from the two mechanisms are due to electrons from very different parts of the electron energy distribution. The highenergy electrons responsible for ICS make essentially no contribution to the HXR bremsstrahlung emission. Similarly, the much lower energy electrons responsible for HXR bremsstrahlung emission contribute no ICS emission. 
Table 5.1. Comparison of fitted HXR/ $\gamma$-ray photon spectral indices

\begin{tabular}{|c|c|c|c|c|c|c|c|c|c|c|c|c|}
\hline \multirow[b]{2}{*}{$\mathrm{keV}$} & \multicolumn{4}{|c|}{ Ultrarelativstic ICS } & \multicolumn{4}{|c|}{ Mildly Relativistic ICS } & \multicolumn{4}{|c|}{ Bremsstrahlung } \\
\hline & ISO & PAN & C-B & $\delta$-B & ISO & PAN & $\mathrm{C}-\mathrm{B}$ & $\delta-\mathrm{B}$ & ISO & PAN & C-B & $\delta-\mathrm{B}$ \\
\hline $20-80$ & 2.0 & 2.0 & 2.0 & 1.0 & 2.6 & 2.2 & 1.8 & 1.4 & 3.5 & 3.2 & 2.5 & 1.9 \\
\hline $200-800$ & 2.0 & 2.0 & 2.0 & 1.0 & 2.1 & 2.1 & 2.1 & 1.1 & 2.9 & 2.5 & 2.0 & 1.3 \\
\hline
\end{tabular}

Note. - ISO: isotropic, PAN: pancake, C-B: cone beam, $\delta$-B: monodirectional beam. The results are calculated based on a single power-law electron energy distributions with a spectral index $\delta=3$. For the mildly relativistic ICS case, an incident photon energy of $2 \mathrm{keV}$ is used. 
Nevertheless, Korchak (1971) compared the two mechanisms for a single isotropic, power-law electron distribution for ICS in the ultra-relativistic limit. This result was recast by Krucker et al. (2008b) as the ratio $R$ of the ICS to EIB emissivities, given approximately as

$$
R=\frac{3}{2 \alpha^{\prime}} \frac{n_{\gamma}}{n_{p}}(2 \delta-1) Q(\delta)\left(\frac{\epsilon_{2}}{4 \epsilon_{1}}\right)^{(1-\delta) / 2}\left(\frac{\epsilon_{2}}{m_{e} c^{2}}\right)^{\delta-1 / 2}
$$

where $n_{\gamma}$ is the number density of ambient (photospheric photons), $n_{p}$ is the number density of protons in the source, $\alpha^{\prime}$ is the fine structure constant, and $Q(\delta)$ is given by

$$
Q(\delta)=\frac{2\left(11+4 \delta+\delta^{2}\right)}{(1+\delta)(3+\delta)^{2}(5+\delta)}
$$

For a photospheric photon density $n_{\gamma} \sim 10^{12} \mathrm{~cm}^{-3}$ and a proton density of $n_{p} \sim$ $10^{8}\left(10^{9}\right) \mathrm{cm}^{-3}$, ICS will exceed EIB in the 10-100 keV range only for very hard electron distributions: $\delta<2.9(2.5)$. In other words, for an isotropic power-law distribution of electrons characterized by a single index $\delta$, ICS is only favored for relatively tenuous plasmas and hard electron distributions. This comparison does not include the effect of an anisotropic electron distribution. For the particular case where $\delta=3$, for favorable viewing geometries, ICS can exceed bremsstrahlung emission by a factor of a few for a pancake distribution, and 12-40 for a cone-beam distribution, allowing the condition on the ambient density and/or $\delta$ to be relaxed somewhat. For example, for a cone-beam anisotropy, ICS exceeds bremsstrahlung over all or part of the $10-100 \mathrm{keV}$ range if $\delta<3.5(3)$ for ambient plasma densities of $10^{8}\left(10^{9}\right) \mathrm{cm}^{-3}$. Alternatively, for $\delta=3$, ICS could still contribute to the $10-100 \mathrm{keV}$ range for an ambient density as high as $3 \times 10^{9} \mathrm{~cm}^{-3}$. It is worth pointing out that if collisions are the primary electron pitch angle scattering mechanism, low-energy electrons may be 
nearly isotropic whereas high-energy electrons could be highly anisotropic, yielding even greater enhancements of ICS relative to EIB than those noted here.

There is no reason to suspect, moreover, that the electron distribution function is necessarily characterized by a single power law over many orders of magnitude in energy. A variety of spectral features in the electron distribution have been reported, including low- and/or high-energy cutoffs (Holman, 2003), upward breaks (a flatter spectrum) above $~ 300-400 \mathrm{keV}$ (e.g., Dennis, 1988; Vestrand, 1988; Rieger \& Marschhäuser, 1991; Trottet et al., 1998; Ackermann et al., 2012), and downward breaks (a steeper spectrum) above energies from $\sim 30-200 \mathrm{keV}$ (e.g., Lin \& Schwartz, 1987; Dulk et al., 1992; Holman et al., 2003; Krucker et al., 2007a). We briefly explore conditions under which spectral breaks may be favorable to ICS emission. To do so we assume a double power-law electron energy spectrum as the input to compare the contribution from ICS and bremsstrahlung emission. For the isotropic case, the electron distribution can be written as

$$
f_{e}\left(\gamma, \Omega_{e}\right)=\frac{1}{4 \pi} \begin{cases}K_{L}(\gamma-1)^{\delta_{L}}, & \gamma_{1} \leqslant \gamma<\gamma_{b} \\ K_{U}(\gamma-1)^{\delta_{U}}, & \gamma_{b} \leqslant \gamma<\gamma_{2}\end{cases}
$$

and $f_{e}\left(\gamma, \Omega_{e}\right)=0$ for $\gamma<\gamma_{1}$ and $\gamma>\gamma_{2}$, where $\gamma_{1}$ and $\gamma_{2}$ correspond to the lowerand upper-cutoff energies $E_{1}$ and $E_{2}$, taken to be $10 \mathrm{keV}$ and $100 \mathrm{MeV}$, respectively; $K_{L}, K_{U}, \delta_{L}$, and $\delta_{U}$ are, respectively, the normalization constants and spectral indices in the lower and upper energy range, separated by a break energy $E_{b}$ (corresponding to $\gamma_{b}$ ). We first consider the case where the spectrum "breaks down" above $E_{b}$, i.e., $\delta_{L}<\delta_{U}$. We fix the upper spectral index $\delta_{U}$ and vary $E_{b}$ and $\delta_{L}$ as free parameters to explore the relative contributions of ICS and thin-target, non-thermal bremsstrahlung to the HXR spectrum. In effect, the electrons below $E_{b}$ largely deter- 
mine the bremsstrahlung contribution whereas those well above $E_{b}$ determine the ICS emission. Smaller values of $\delta_{L}$ and/or larger values of $E_{b}$ reduce the bremsstrahlung contribution relative to the ICS contribution.
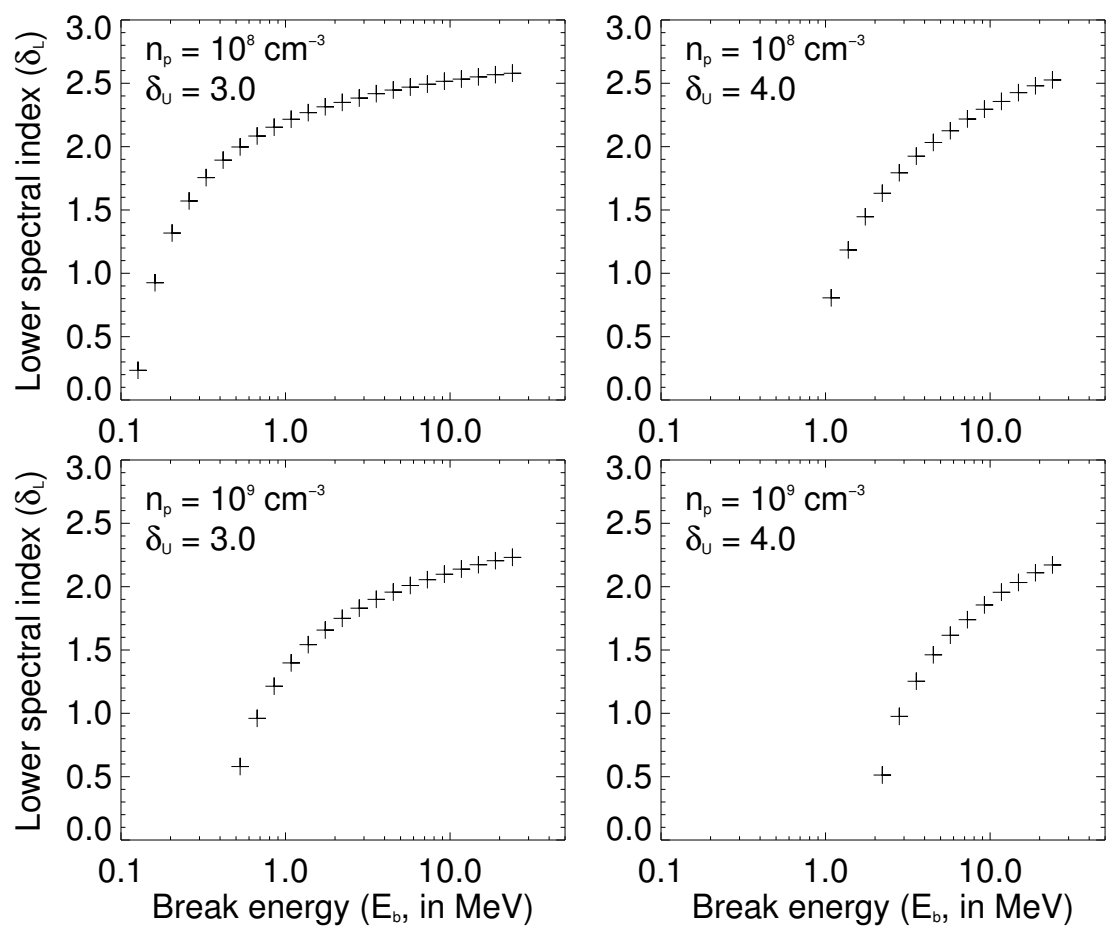

Fig. 5.13. - Comparison of ICS and bremsstrahlung emission from the same double power-law spectrum that breaks down (steepens) at higher energies. Combinations of the break energy $E_{b}$ and lower spectral index $\delta_{L}$ for a given upper spectral index $\delta_{U}$ are shown to equalize the integrated ICS and bremsstrahlung photon energy from $10 \mathrm{keV}$ to $100 \mathrm{keV}$. The ICS wins over the bremsstrahlung emission in the lower right region (larger $E_{b}$ and smaller $\delta_{L}$ ) and vice versa.

To illustrate this we return to ICS from photospheric photons with a monoenergetic energy $2 \mathrm{eV}$ and a number density of $10^{12} \mathrm{~cm}^{-3}$ scattering on relativistic electrons. The ambient ion density is assumed to be $10^{8} \mathrm{~cm}^{-3}$ (upper panels) and $10^{9} \mathrm{~cm}^{-3}$ (lower panels). A combination of $\delta_{L}$ and $E_{b}$ is found for each given $\delta_{U}$ by equalizing the integrated ICS and bremsstrahlung photon emissivities from 
$10 \mathrm{keV}$ to $100 \mathrm{keV}$. In Figure 5.13, it is seen that ICS dominates over thin-target bremsstrahlung emission in the lower right region (larger $E_{b}$ and smaller $\delta_{L}$ ) and thin-target bremsstrahlung dominates in the upper left region. We also notice that for a softer spectrum at high energies (a larger $\delta_{U}$ ), the condition of equality moves to the lower right corner of the two-dimensional parameter space, which means a larger $E_{b}$ or a smaller $\delta_{L}$ is required. A large value of $E_{b}$ increases the relative number density of higher energy electrons, increasing the contribution of ICS emission; whereas a lower value of $\delta_{L}$ reduces the relative number of low-energy electrons leading to a corresponding reduction in bremsstrahlung emission.

As noted above, in some cases the electron energy distribution is better characterized by a broken power law where $\delta_{U}<\delta_{L}$; that is, the spectrum "breaks up" above $E_{b}$. In these cases, the electron spectrum hardens above energies of a few $\times 100 \mathrm{keV}$ and then rolls over to a steeper distribution at energies of several $\mathrm{MeV}$ to several tens of $\mathrm{MeV}$ (e.g., Ackermann et al., 2012). If the flat spectral component indeed extends to $10 \mathrm{~s}$ of $\mathrm{MeV}$, ICS can quite easily exceed bremsstrahlung emission in the photon energy range of a few $\times 100 \mathrm{keV}$ and above, i.e., for continuum $\gamma$-ray emission. Even for an ambient density of $10^{10} \mathrm{~cm}^{-3}$, ICS exceeds bremsstrahlung for $\delta<2.5$.

For the case of HXR photons resulting from EUV/SXR photons upscattered by mildly relativistic electrons, it is more difficult to assess the relative contributions of ICS and bremsstrahlung emission. It is no longer possible to treat the photon density distribution as monoenergetic, an approximation that is justified only when $\epsilon_{2} \gg \epsilon_{1}$. It is instead necessary to integrate over the photon energy distribution which, in turn, depends on the nature of the flare in question. Consideration of ICS in the mildly relativistic regime must therefore be assessed on a case-by-case basis. We briefly consider two specific cases in the next section but detailed comparisons between the 
two mechanisms in the mildly relativistic regime are beyond the scope of this paper.

\subsection{Application to Coronal HXR Observations}

In this section, we briefly consider selected observations. First, however, we consolidate and summarize our results.

1. In the first part of Section 5.2.1, we computed HXR spectra resulting from ICS of photospheric photons on an isotropic distribution of ultrarelativistic electrons. A power-law distribution of electrons with an index $\delta$ results in the well-known power-law spectrum of upscattered photons with an index $\alpha=$ $(\delta+1) / 2$. We find that the ICS photon flux increases with the heliographic longitude $\lambda$ of the source. If the electron spectrum has a high-energy cutoff, the photon spectrum has a corresponding high-energy cutoff energy. The highenergy cutoff in the photon spectrum also varies with $\lambda$ : the cutoff increases with increasing longitude to a maximum of $\approx 4 \gamma_{\max }^{2} \epsilon_{1}$. For a source at a fixed longitude, the high-energy cutoff in the photon spectrum has no dependence on $\delta$.

2. In the second part of Section 5.2.1, we computed HXR spectra resulting from ICS of isotropic EUV/SXR photons on an isotropic distribution of mildly relativistic electrons - those with energies of order a few $\times 100 \mathrm{keV}$ to a few $\mathrm{MeV}$. We find that, while the HXR spectra are qualitatively similar to those resulting from ICS on ultrarelativistic electrons, the photon spectra are significantly steeper than $(\delta+1) / 2$. In particular, for $0.2 \mathrm{keV}$ photons incident on isotropic electrons with $\delta=2,3,5$, we find that the spectral indices of the upscattered photons are 1.6, 2.2, and 3.3, respectively; for $2 \mathrm{keV}$ incident photons the photon 
indices are $1.9,2.6$, and 4.2 , respectively.

3. In Section 5.2.2, we considered ICS of an isotropic distribution of photons on an anisotropic distribution of electrons in the relativistic regime. The most extreme case is that of a monodirectional ( $\delta$-function) beam directed along the LOS, which results in a photon spectrum with an index $\alpha=(\delta-1) / 2$, harder by 1 than the HXR photon spectral index $\alpha=(\delta+1) / 2$ resulting from ICS on an isotropic distribution of electrons; moreover, the photon flux is roughly four to five orders of magnitude greater than that resulting from ICS of isotropic photons on isotropic electrons. More realistic are cone-beam or pancake distributions of electrons with finite angular widths. These both result in photon spectra with indices similar to the case of scattering on an isotropic electron distribution. For a favorable viewing geometry, the cone beam yields an HXR photon flux that is enhanced by a factor $\sim 4 / \Delta \theta_{b}^{2}$ over the isotropic case whereas a pancake distribution yields an enhancement of $\sim 1 / \Delta \theta_{p}$ for small $\Delta \theta_{b}$ and $\Delta \theta_{p}$ for a fixed electron number density.

4. We considered the case of isotropic photons scattering on an anisotropic distribution of mildly relativistic electrons in Section 5.2.2. We again considered a monodirectional beam, a cone beam, and a pancake anisotropy and compared them to the case of ICS on isotropic electrons. The monodirectional beam produces an ICS HXR spectrum that is again quite hard (indices of 1.2 and 1.4 for incident photons of 0.2 and $2 \mathrm{keV}$, respectively, for $\delta=3$ ), but not as hard as the ultrarelativistic case (photon index of 1.0 for $\delta=3$ ). The HXR photon flux is enhanced by 2.5-3.5 orders of magnitude over the isotropic case when EUV photons are scattered and by 1-2.5 orders of magnitude when SXR photons are scattered. HXR spectra resulting from a cone beam show a broken power-law 
structure in which the spectrum transitions from a slope similar to the monodirectional beam case to a slope similar to the isotropic case and are enhanced by $\approx 2$ orders of magnitude relative to the isotropic case. HXR spectral slopes resulting from ICS on a pancake electron anisotropy in the mildly relativistic regime depart more subtly from the isotropic case and are enhanced by $\lesssim 1$ order of magnitude relative to the isotropic case.

5. In Section 5.3, we review properties of thin-target EIB and EEB emission and calculate the emission from both isotropic and anisotropic electron distributions. The directivity of thin-target, non-thermal bremsstrahlung is less than that of ICS. By assuming the same kinds of anisotropic electron distributions as above, we found that the resulting EIB spectrum gets harder with increasing degrees of anisotropy, but the enhancement is less than that found for ICS emission, especially at low HXR photon energies. The EEB bremsstrahlung emission can be safely neglected for photon energies $\lesssim 100 \mathrm{keV}$ for an isotropic electron distribution, but becomes more important for fast electrons with higher anisotropies. The EEB emission has a stronger directivity than the EIB, but is still less directive than the ICS emissivity. Including the EEB component leads to a flattening of the HXR spectrum at high photon energies. For high degrees of anisotropy, the emissivity can be nearly doubled when including the EEB contribution.

6. We compared the contributions of ICS and thin-target non-thermal bremsstrahlung to coronal HXR emissions (10-100 keV) in Section 5.3 for photospheric photons scattered on a simple isotropic power-law distribution of electrons. We then showed that a double power-law electron distribution that breaks down (steepens) at higher energies with a sufficiently large break energy $E_{b}$ and/or a flat 
spectrum at lower energies can result in an excess of ICS emission relative to bremsstrahlung emission. An electron distribution that breaks up (flattens) at higher energies can yield ICS that exceeds bremsstrahlung emission rather easily for photon energies more than a few $\times 100 \mathrm{keV}$. ICS emission resulting from EUV/SXR photons scattering on mildly relativistic electrons depends on the details of the photon spectrum and must be computed on a case-by-case basis and compared with the corresponding bremsstrahlung emission.

We now briefly discuss observations of several coronal HXR and continuum $\gamma$-ray sources and consider whether ICS may play a role in the observed emission.

\subsubsection{Coronal $\gamma$-Ray Sources from Three Powerful Flares}

Observations of coronal continuum $\gamma$-ray $(200-800 \mathrm{keV})$ sources associated with three extremely powerful X-class flares were presented by Krucker et al. (2008a). The flares occurred on 2003 October 28 (>X17; disk center), 2005 January 20 (X7.1; W61), and 2005 September 7 (>X17; solar limb). Early in each event, footpoint emission dominated the continuum $\gamma$-ray emission. Later, during the (exponential) decay phase of the $\gamma$-ray emission, the coronal source became more prominent than the footpoint emission. The photon spectral index of the coronal source in each case was significantly harder $(\alpha \approx 1.5-2)$ than that of the footpoint sources $(\alpha \approx 3-4)$. The authors suggest that the coronal sources result from non-thermal bremsstrahlung emission. The photon spectral indices are at, or near, the theoretical limit of nonthermal bremsstrahlung emission and require a non-thermal electron distribution with a low-energy cutoff $E_{c}>1 \mathrm{MeV}$ in all cases (Brown et al., 2008); see also Section 5.3. Given that the electron transit time of $\mathrm{MeV}$ electrons is much shorter than their collisional loss time for typical coronal conditions in a flare, electron trapping is needed 
to produce the coronal source. In the specific example of the flare on 2005 January 20, Krucker et al. (2008a) suggest that synchrotron losses dominate electron energy losses and the observed $\gamma$-ray emission between 200 and $800 \mathrm{keV}$ can be explained by bremsstrahlung emission from electrons with energies $>E_{c}=8 \mathrm{MeV}$. If the trap is stable, the emission becomes thick target and the total energy in $>8 \mathrm{MeV}$ electrons is estimated to be $\sim 10^{28} \mathrm{erg}$. It should be noted that while EEB emission contributes to this photon energy range ( $\sim 20 \%$ of the total emission for the isotropic case), the net effect is to harden the photon spectrum somewhat. However, for a highly anisotropic electron spectrum, EEB could be responsible for a larger fraction of the continuum $\gamma$-ray emission for a favorable viewing geometry.

MM10 considered the continuum $\gamma$-ray emission from the flare on 2005 January 20. They find that ICS represents a viable alternative to bremsstrahlung emission in the sense that only a modest number of ultra-relativistic electrons are needed to account for the observed continuum $\gamma$-ray source in terms of ICS. In particular, they estimate that as long as an (isotropic power-law) electron energy distribution function extends to $>100 \mathrm{MeV}$, a total of $\sim 10^{31}$ electrons with energies $>0.5 \mathrm{MeV}$ are sufficient to account for the source. With a source volume of $\sim 5 \times 10^{28} \mathrm{~cm}^{-3}$ and an ambient density of $10^{8} \mathrm{~cm}^{-3}$, a number density of just $200 \mathrm{~cm}^{-3}>0.5 \mathrm{MeV}$ electron is needed, or a fraction of only $2 \times 10^{-6}$ of the ambient electrons. As noted in Section 5.2.1, an error in the analysis of MM10 resulted in over optimistic ICS emissivities. Our calculations lead to a revised estimate for the total number of electrons required to account for the observed $\gamma$-ray emission reported by Krucker et al. (2008a) for the 2005 January 20 flare that is a factor $\sim 500$ larger $\left(10^{5} \mathrm{~cm}^{-3}\right)$ which, if the electron energy distribution indeed extends continuously to $\sim 100 \mathrm{MeV}$, implies that a fraction of $0.1 \%$ of the ambient electrons are accelerated to high energies. If the electrons responsible 
for the emission are trapped near the loop top, they will have a pancake-like anisotropy which, as shown in Section 5.2.2, requires fewer electrons for a favorable viewing geometry. If this is the case, the required number of electrons can perhaps be reduced to $(1-2) \times 10^{4} \mathrm{~cm}^{-3}$ electrons, again normalized to a reference energy of $0.5 \mathrm{MeV}$.

As noted above, Krucker et al. find that the energy content of the electrons $>E_{c}=8 \mathrm{MeV}$ needed to account for the observed emission in terms of bremsstrahlung emission is $\sim 10^{28} \mathrm{erg}$ in total. The implied number of energetic electrons required to account for the source in terms of bremsstrahlung emission, normalized to $0.5 \mathrm{MeV}$ in order to compare it to ICS, is $\sim 2 \times 10^{6} \mathrm{~cm}^{-3}$, which is $20-100$ times the number of fast electrons needed for ICS, assuming a single power-law distribution with $\delta=3$. The ratio likewise holds for the energy content of fast electrons. The index of the electron distribution function must lie in the range $\delta=2-3$ to account for the continuum $\gamma$-ray emission in terms of ICS. We conclude our discussion of the continuum $\gamma$-ray events by suggesting that ICS of photospheric photons on relativistic electrons can account for the observed emission. It is energetically more favorable than bremsstrahlung by a factor of 20 (isotropic) to 100 (pancake anisotropy).

\subsubsection{HXR Emission from High in the Corona}

An intriguing example of a coronal HXR source for which ICS may have played a role is that reported by Krucker et al. (2007b). A powerful flare occurred in AR 10180 when it was $40^{\circ}$ behind the limb on 2002 October 27, as viewed from Earth. The flare was observed directly from Mars by the Gamma-Ray Spectrometer on board the Mars Odyssey mission. On this basis it was estimated to be comparable in SXR class to the flares on 2003 October 28 and 2005 January 20 discussed in the previous section. A large, diffuse HXR source was observed by RHESSI. Both the 
footpoints and the thermal flare loops were occulted for this event - in fact, the source was high in the corona: the radial occultation height is $300^{\prime \prime} \pm 65^{\prime \prime}$ and the source centroid was $\approx 80^{\prime \prime}$ above limb, implying a source height of $\sim 0.3-0.4 R_{\odot}$. The HXR spectrum was observed up to $60 \mathrm{keV}$. The photon spectral index was relatively hard, changing systematically from 3.5 to 3 during the exponential decay phase of the event, implying an electron spectral index $\delta=2.5$ to 3 . Based on Figure 3 in Krucker et al. (2007b), in which the HXR spectrum is presented for a time during the decay phase, we estimate the photon flux at $30 \mathrm{keV}$ to be 0.1 photons $\mathrm{s}^{-1} \mathrm{~cm}^{-2} \mathrm{keV}^{-1}$. When interpreted in terms of non-thermal bremsstrahlung emission, the authors estimate that the instantaneous number density of electrons $>10 \mathrm{keV}$ required $\sim 10^{7} \mathrm{~cm}^{-3}$, or $\sim 10 \%$ of the total electron number density for an ambient plasma density of $\sim 10^{8}$ $\mathrm{cm}^{-3}$. The lower limit to the total energy in non-thermal electrons is $2 \times 10^{29} \mathrm{erg}$.

Is ICS a viable alternative for this event? For the case of ultrarelativistic electrons the photon spectral index of $\alpha=3.1$ implies that an electron spectral index is $\delta=5.2$. When normalized to $0.5 \mathrm{MeV}$, a large number of energetic electrons are required, $\sim 3 \times 10^{7} \mathrm{~cm}^{-3}$, containing $10^{31} \mathrm{erg}$. We note, however, that the electrons involved in ICS in this case have energies $\gtrsim 20 \mathrm{MeV}$. The minimum required number density of electrons with energies $>20 \mathrm{MeV}$ is far more modest, $n_{\min } \sim 10 \mathrm{~cm}^{-3}$, containing $\sim 2 \times 10^{26} \mathrm{erg}$. An anisotropic electron distribution brings these estimates down yet further. Of course, ICS depends entirely on the details of the electron distribution function. If the electron distribution is described by a single power law $(\delta=5.2)$ that extends below $\sim 2 \mathrm{MeV}$, then it becomes energetically unfavorable compared to bremsstrahlung. In the context of a double power law with $\delta_{U}=5.2$, the break energy - or low-energy cutoff - would need to be a few MeV in order for ICS to be relevant. Consideration of an anisotropic electron distribution can bring the cutoff 
down to $E_{b} \lesssim 500 \mathrm{keV}$

We now consider an alternative to ICS in the relativistic regime and instead consider a mildly relativistic distribution of electrons. In Section 5.2, we showed that the upscattered photon spectrum resulting from mildly relativistic electrons is significantly steeper than that from the classic relation $(\delta+1) / 2$ at small values of $\epsilon_{2} / \epsilon_{1}$. In the mildly relativistic regime, however, monoenergetic photons are no longer appropriate as an approximation for the incident photon spectrum. Therefore, as a crude estimate, we use the simulated flare spectrum produced by the CHIANTI software package (Dere et al., 1997, 2009) based on an M2 flare in the EUV/SXR energy range (0.1-20 keV, see the left panel of Figure 5.14) and scale the photon number density up to represent a large X-class flare. The EUV/SXR spectrum declines rapidly with energy above $1-2 \mathrm{keV}$ and so the redistribution of photons by downscatter can be neglected for the HXR energy range in question. After some experimentation, we find that a double power-law electron energy distribution with an upper spectral index of $\delta_{U}=3.8$ produces an upscattered HXR spectrum with the observed photon spectral index of $\alpha=3.1$ (the middle panel of Figure 5.14, blue solid curve). The electron distribution is assumed to be isotropic at all energies. The spectrum has a break energy at $300 \mathrm{keV}$ with a flatter spectral index at lower energies $\left(\delta_{L}=1.5\right)$. An ambient density of $10^{8} \mathrm{~cm}^{-3}$ has been assumed and the number density of energetic electrons with $\delta_{U}=3.8$ is $3 \times 10^{3} \mathrm{~cm}^{-3}$, normalized to $0.5 \mathrm{MeV}$, containing $\approx 4 \times 10^{27}$ ergs - a factor $\approx 50$ less than that implied by the bremsstrahlung interpretation. The electron distribution yields EIB bremsstrahlung shown as the red solid curve in the middle panel of Figure 5.14. The EIB photon spectrum is significantly flatter than the ICS spectrum and becomes comparable to ICS at photon energies of $\gtrsim 60 \mathrm{keV}$. The dashed lines show the ICS and EIB emission from a cone-beam (half-width of 
$10^{\circ}$ ) distribution of electrons. In this case, ICS clearly exceeds EIB by a large factor and the estimates of the required number density of fast electrons and their energy content is reduced by a corresponding factor. We acknowledge that the photon field resulting from a flaring active region is no longer well approximated by an infinite plane (as was employed for the photospheric photon field). However, since ICS is dominated by large-angle scattering, the error introduced is not large. We conclude that ICS from mildly relativistic electrons may make a significant contribution to the observed HXR emission in this case. Again, a broken power-law electron spectrum is required with a break energy of $300 \mathrm{keV}$.

\subsubsection{Electron Acceleration in a "Masuda"-like Event}

Another example of an intriguing coronal HXR source is the limb-occulted flare on 2007 December 31 (Krucker et al., 2010). The flare was $\approx 12^{\circ}$ over the limb as observed by RHESSI. However, comparing direct observations by the X-ray Spectrometer on board the Mercury MESSENGER spacecraft with GOES observations showed that it was an M2 SXR flare. The flare shared many attributes with the "Masuda" flare (Masuda et al., 1994), a flare that showed an HXR source above the thermal SXR loops. The coronal HXR spectrum of the 2007 December 31 event is clearly nonthermal with a photon spectral index 4.2 between 20 and $80 \mathrm{keV}$. In order for the thin-target bremsstrahlung model to account for the observed HXR flux, the number density of non-thermal electrons above $16 \mathrm{keV}$ is required to be nearly the same as the ambient electron density $\left(\sim 2 \times 10^{9} \mathrm{~cm}^{-3}\right)$. In other words, essentially all the electrons in the HXR source are accelerated to energies $>16 \mathrm{keV}$ !

To account for the HXR spectrum in terms of ICS in the ultra-relativistic regime, a soft power-law index $\delta=7.4$ is required. With an observed HXR photon flux of $\approx 0.2$ 

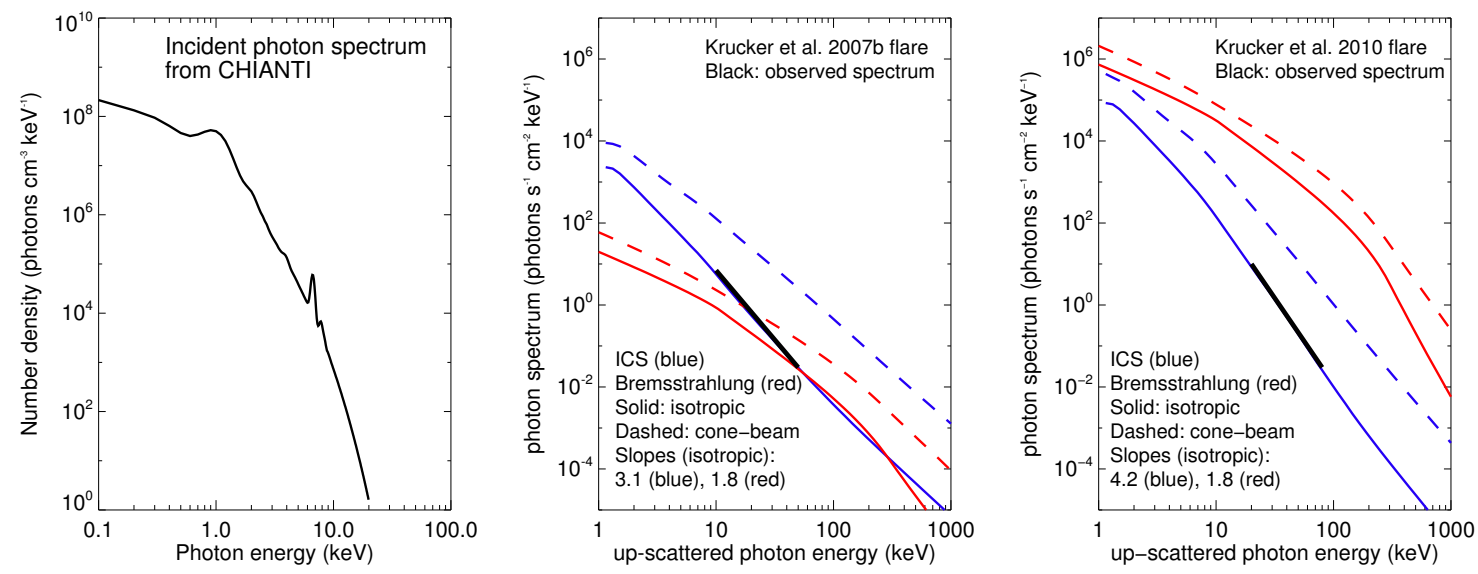

Fig. 5.14.- Left: the simulated EUV/SXR spectrum of a flare from CHIANTI (based on an M2 flare). Middle: HXR photon spectra as observed at 1 AU from the ICS (blue) and EIB (red) emission, respectively, to account for the Krucker et al. (2007b) flare. The photon spectrum has been scaled up to represent an X10 flare. The electron energy spectrum is assumed to be a double power law extending from $10 \mathrm{keV}$ to $100 \mathrm{MeV}$. The spectrum has a break energy of $300 \mathrm{keV}$, with spectral indices of 1.5 and 3.8 at the lower and higher energies, respectively. Solid and dashed lines are for an isotropic electron distribution and a cone-beam distribution with a half-width of $\theta_{b}=10^{\circ}$. The heavy black line represents the observed HXR spectrum. Right: HXR photon spectra from the ICS (blue) and EIB (red) emission, to account for the Krucker et al. (2010) flare. The electron energy spectrum has parameters similar to the middle panel, except that the spectral index at higher energies $(>300$ $\mathrm{keV})$ is now $\delta_{U}=5.2$. No scaling is applied to the simulated incident photon number density since the flare was itself an M2 class flare.

photons $\mathrm{s}^{-1} \mathrm{~cm}^{-2} \mathrm{keV}^{-1}$ at $50 \mathrm{keV}$ and source volume of $\sim 10^{27} \mathrm{~cm}^{3}$ (see Table 1 of Krucker et al., 2010), we find that the number density of (isotropic) electrons needed is unacceptably large: $3 \times 10^{16}$ electrons $>0.5 \mathrm{MeV}$. The electrons relevant to ICS in this regime are again those with energies $\gtrsim 20 \mathrm{MeV}$, for which we find $n_{\min } \sim 2 \times 10^{6} \mathrm{~cm}^{-3}$. Even so, in the context of a double power-law electron distribution, ICS becomes energetically unfavorable compared to EIB emission for break energy $E_{b} \lesssim 15 \mathrm{MeV}$ even with an anisotropic electron distribution.

Turning to ICS in the mildly relativistic regime, we have again used the simulated 
M2 flare spectrum produced by CHIANTI. This time, however, it is not necessary to scale the flare up since the event in question was itself an M2 flare. Again considering a broken power law with $E_{b}=300 \mathrm{keV}$ and $\delta_{L}=1.5$, we find that an electron energy distribution with a spectral index $\delta_{U}=5.2$ produces an up-scattered HXR spectrum with the observed index $\alpha=4.2$ over the $20-80 \mathrm{keV}$ range (the right panel of Figure 5.14, blue solid curve). We have again ignored the finite size of the flaring active region but note that, unlike the case discussed in the previous subsection, the HXR source is relatively low in the corona and just $6 \mathrm{Mm}$ above the SXR loops. The number density of energetic electrons above $0.5 \mathrm{MeV}$ required to account for the HXR source is $\sim 6 \times 10^{8} \mathrm{~cm}^{-3}$ for the isotropic case, which is $\approx 30 \%$ of the ambient density. The total energy in mildly relativistic electrons is $\sim 3 \times 10^{29} \mathrm{erg}$, comparable to that estimated for the bremsstrahlung interpretation ( $\left.\gtrsim 10^{29} \mathrm{erg}\right)$. However, the bremsstrahlung emission produced by the model electron spectrum in this case exceeds the ICS contribution by orders of magnitude (the right panel of Figure 5.14, red solid curve). This is a result of the much lower EUV/SXR photon number density for this flare, the higher ambient density, and the relatively soft electron spectrum above the cutoff. We conclude that ICS in the mildly relativistic regime cannot account for the coronal HXR source.

\subsection{Concluding Remarks}

We have considered three specific cases of coronal HXR and continuum $\gamma$-ray sources. In the case of the continuum, flat-spectrum, $\gamma$-ray sources produced by the powerful flares described by Krucker et al. (2008a), we are in qualitative agreement with MM10 that ICS in the ultrarelativistic regime can account for the observed photon spectrum. In the case of the flare on 2002 October 27 described by Krucker et al. (2007b), the observed photon spectrum is somewhat softer. Either ultrarelativistic or mildly 
relativistic ICS may account for the observations, but an electron distribution that breaks down from a flat distribution to a steeper distribution is required in both cases. Finally, we considered the "Masuda"-like flare reported by Krucker et al. (2010). Here, ICS is unlikely to play a role in the relativistic case unless rather large numbers of ultrarelativistic electrons are accelerated and a high break energy is assumed. ICS in the mildly relativistic regime fails to account for the HXR emission in this case.

We conclude that ICS may play a role in certain coronal HXR or continuum $\gamma$ ray sources. Such sources require rather special conditions for ICS to prevail over bremsstrahlung emission. In particular, one or more of the following conditions must be met: (1) the ambient plasma density is low, (2) the electron energy distribution is complex - a double power law or similar, (3) the electron angular distribution is anisotropic, and (4) the flare produces enough EUV/SXR photons for the mildly relativistic ICS to be effective. Sources in which ICS plays a significant role are likely rare. Potential cases must be analyzed in some detail.

Such analyses would be greatly aided by the availability of imaging and spectroscopic data at both $\mathrm{HXR} / \gamma$-ray energies and centimeter/millimeter radio wavelengths. We note that limited microwave observations are available for the event discussed in Section 5.4.3; Krucker et al. (2010), who find that the radio emission is broadly consistent with the bremsstrahlung interpretation, point out that the electrons that produce the $17 \mathrm{GHz}$ emission have energies of order $1.2 \mathrm{MeV}$. These are the same mildly relativistic electrons that could be responsible for ICS, if it is relevant, in the mildly relativistic regime. It is well known that a close relationship between ICS and synchrotron emission exists for isotropic distributions of electrons and photons. The ratio of the synchrotron power $P_{\text {synch }}$ to the ICS power $P_{\mathrm{ICS}}$ is equal to the ratio of the magnetic energy density $u_{B}=B^{2} / 8 \pi$ and the photon energy density $u_{\mathrm{ph}}$ : 


$$
\frac{P_{\mathrm{synch}}}{P_{\mathrm{ICS}}}=\frac{u_{B}}{u_{\mathrm{ph}}}
$$

One could in principle use joint radio and HXR observations to determine whether ICS in the mildly relativistic regime is relevant. Given that anisotropic electron distributions may be an important element in determining the relevance of ICS, the above relationship would need to be recast to the specifics of the electron anisotropy. It is beyond the scope of this paper to investigate whether the HXR and radio observations of flares can be reconciled in the framework of mildly relativistic ICS, but it is important to emphasize that the same electrons are responsible for the radio emission and the photon up-scatter in this case and both mechanisms would jointly impose strong constraints on the electron distribution. 
Chapter 6

Summary and Future Work 
In this dissertation, radio and X-ray studies have been explored as diagnostics of magnetic energy release processes in solar flares. Here I summarize the major results of each chapter and outline some future projects on this topic.

\subsection{Summary}

In Chapter 1, I started with a description of general aspects of the Sun and solar flares. Some historical background leading to the current framework of the standard flare scenario was then reviewed. Due to the complex and dynamic nature of solar flares, the need for exploiting multi-wavelength studies was emphasized. I focused on studies in radio and X-ray wavelengths because these emissions are sensitive to most sources of energetic electrons produced by flares. I then reviewed radio and X-ray emission mechanisms in association with flares and outlined their diagnostic uses.

Chapter 2 presents a detailed study of a particular type of solar coherent radio burst-zebra-pattern bursts. Spatially resolved radio dynamic spectroscopic observation provided by a three-element array - the FST — was first used to characterize the spatial, time, and spectral properties of these bursts. Based on these observations, we obtained their absolute source locations on the solar disk as a function of time and frequency, which allowed us to examine the relevant emission mechanisms. We conclude that the double plasma resonance (DPR) model - a model based on enhanced plasma instabilities (due to electron cyclotron maser emission) occurring at resonance layers for which the upper-hybrid frequency $\nu_{\mathrm{uh}}$ is harmonically related to the electron gyrofrequency $\nu_{\mathrm{ce}}$-is most favorable since it can explain most features of this burst event. Based on the DPR model, we were able to derive various plasma parameters in the emission source, including the plasma density and magnetic field strength, as well as their variation over coronal height. With the help of a nonlinear force-free 
magnetic-field extrapolation from the observed photospheric vector magnetogram, we placed the burst source in a three-dimensional magnetic field configuration. We suggest that the emission source was located in a post-flare/post-CME loop system in which the parent energetic particles were possibly supplied by an energy release site high up in the corona above the radio source, perhaps the result of magnetic reconnections induced by the associated fast halo CME.

In Chapter 3, the "solar mode" commissioning of the recently upgraded VLA is described. Although the $V L A$ is a general-purpose radio telescope optimized to observe faint cosmic radio sources, provisions have been made to allow solar observing. Yet the hardware should be carefully tested for effective observing and data calibration. Based on the test results, we conclude that the current system of the $V L A$ has a dynamic range sufficient to observe the quiet Sun, active regions, and moderate solar flares, but will suffer significant compression when observing flares with a flux density over a few $\times 100 \mathrm{sfu}$. The $V L A$ allows the usage of standard cosmic radio sources as references for solar data calibration, providing that $20 \mathrm{~dB}$ solar attenuators are inserted into the signal path when observing the Sun for gain reduction and are switched out when observing the calibrators. We found that the $20 \mathrm{~dB}$ solar attenuators have well-defined amplitude and phase properties that can be easily compensated in calibration. But the net phases introduced by the T304 step attenuators are much more complicated and difficult to characterize. Therefore we employed a "set-and-remember" scheme for solar observing: the T304 attenuator were optimized in the first solar scans and remembered for the rest of the observations. After commissioning, the $V L A$ can observe the Sun over a large frequency bandwidth with simultaneously high spatial, time, and spectral resolutions, thereby enabling broadband dynamic imaging spectroscopy. 
The $V L A$ commissioning work described in the previous chapter led to the first solar observations with the new technique of broadband dynamic imaging spectroscopy, which were discussed in Chapter 4. A rich variety of $\mathrm{dm}-\lambda$ coherent radio bursts were recorded during our VLA RSRO Program, including type IIIdm bursts, fiber bursts, spike bursts, zebra-pattern bursts, etc. Chapter 4 presents a study of type IIIdm bursts - radio signatures of propagating electron beams in the solar corona accelerated from the energy release site - associated with a coronal jet event. Dynamic imaging spectroscopy allowed trajectories of these electron beams to be deduced. Together with magnetic, EUV, and HXR data, we found that the observations were consistent with the standard flare scenario, in which beams emanate from a coronal energy release site and propagate along magnetic field lines both upward and downward. The observed type IIIdm bursts were associated with the upward propagating beams with a velocity of $\sim 0.3 c$, which are accelerated from an energy release site located at a height of $<15 \mathrm{Mm}$ in the low corona. These beams propagated along discrete, over-dense (by roughly an order of magnitude greater than the surrounding medium), ultra-fine magnetic strands (with diameters $<10 \mathrm{~s}$ of $\mathrm{km}$ ) into the upper solar atmosphere. We concluded that the magnetic energy release process is highly fragmentary in nature. The spatial scales within the energy release site are likely 10 $\mathrm{s}$ of $\mathrm{km}$ or less.

Finally, in Chapter 5, we investigated the emission mechanisms for producing the observed HXR (and continuum $\gamma$-ray) sources in the solar corona associated with the impulsive phase of certain solar flares. These HXR/ $\gamma$-ray sources have long been interpreted in terms of thin-target, non-thermal bremsstrahlung emission. However, this interpretation has led to rather extreme physical requirements in some cases, e.g., in one case, essentially all of the electrons in the source must be accelerated to 
non-thermal energies to account for the observed HXR flux from the coronal source. This work reconsidered the role of ICS as an alternative emission mechanism. We investigated two scenarios: (a) photospheric photons upscattered to $\mathrm{HXR} / \gamma$-ray energies by ultrarelativistic electrons which are produced during powerful flares, and (b) EUV/SXR photons upscattered to HXR energies by mildly relativistic electrons which are generally produced in much greater numbers in flares of all sizes. The latter (which was largely overlooked in the past) was shown to produce significant steeper HXR photon spectra than those resulting from ICS on ultrarelativistic electrons. We then explored ICS on anisotropic electron distributions and showed that the resulting emission can be significantly enhanced over an isotropic electron distribution for favorable viewing geometries. We also reviewed results from the thin-target nonthermal bremsstrahlung emission for cases on both isotropic and anisotropic electron distributions, and reconsidered circumstances under which bremsstrahlung or ICS would be favored. We then considered a selection of coronal $\mathrm{HXR} / \gamma$-ray events and found that in some cases the ICS is a viable alternative emission mechanism.

\subsection{Future Work}

Solar radio astronomy has just entered a new era with the new observing technique of broadband dynamic imaging spectroscopy being available with the $V L A$. We have observed a rich variety of $\mathrm{dm}-\lambda$ coherent radio bursts in our $V L A$ RSRO program, some of which were shown in Chapter 4.2. More VLA time was granted for coherent radio burst observations in spring 2013, with an improved instantaneous frequency coverage (from 1 to $4 \mathrm{GHz}$ ) using the recently available subarray observing mode. It is important to note that $\mathrm{dm}-\lambda$ coherent radio bursts are produced by plasmas with a density from $\sim 10^{9}$ to $10^{11} \mathrm{~cm}^{-3}$, characteristic of the low corona and heights 
where energy release is likely to occur. For this reason, these observations have a rich diagnostic potential for energy release in solar flares. I list examples of possible future studies in this field as below:

- By tracing the type-IIIdm-burst-emitting electron beams using dynamic imaging spectroscopy, the magnetic field lines connecting directly to the energy release site can be mapped, which can be used to understand the magnetic field configuration leading to flare energy release.

- By tracing locations of bi-directional type III radio bursts in time and frequency, which are produced by upward and downward propagating electron beams emanating from a common energy release site, the energy release site can be pinpointed.

- With the newly available spatial information at each pixel of the dynamic spectra, relevant emission mechanisms of various types of coherent radio bursts and their connections to the flare energy release can be clarified. Of particular interest are the dm- $\lambda$ spike bursts. They have been previously suspected to be direct signatures of intermittent accelerations in the primary flare energy release site (Benz, 1985, 1986), but recent spatially resolved observations have suggested that they are typically located at much higher altitudes from main flare locations (Benz et al., 2002; Battaglia \& Benz, 2009). Yet these observations were made at relatively low decimetric frequencies $(<450 \mathrm{MHz})$. Imaging spectroscopic observations of these bursts at higher frequencies - hence higher plasma densities and lower coronal heights - may lead to new insights of these bursts. A follow-up study on zebra-pattern bursts is also planned with the true dynamic imaging spectroscopic data, which will provide an improved fidelity of 
locating the source centroid as a function of time and frequency comparing to those in Chapter 2.

- Spatially resolved dynamic spectral observations can be used to diagnose plasma properties in the source region. In particular, the plasma density can be directly derived from the observed emission frequency for plasma radiation. Based on appropriate emission models, other parameters including magnetic field strength, temperature and their variation over coronal heights can be derived. These parameters can be used in association with those derived from observations in other wavelengths as, for example, critical inputs for numerical modeling efforts.

Broadband dynamic imaging spectroscopy enabled by the upgraded $V L A$ in $\mathrm{dm} / \mathrm{cm}-$ $\lambda$ will provide rich diagnostics beyond those listed above from coherent radio bursts. These include coronal magnetography of active regions using gyroresonance absorption and thermal bremsstrahlung diagnostics (see, e.g., Lee 2007 for a review), and deriving magnetic field as well as electron energy spectrum in flaring loops using gyrosynchrotron emission (e.g., Gary et al., 2013).

In addition to the $V L A$, more radio instruments are currently being constructed or planned to enable dynamic imaging spectroscopy in various ranges of frequencies. For example, the Expanded Owens Valley Solar Array (EOVSA; Gary et al., 2011) is a solar-dedicated 13-antenna array currently under construction. Once completed (expected in late 2013), it will have the capability of doing dynamic imaging spectroscopy with an instantaneous bandwidth of $500 \mathrm{MHz}$ sweeping over the $1-18 \mathrm{GHz}$ band with an 1-s cadence. The Frequency Agile Solar Radiotelescope (FASR; Bastian, 2003) is a next-generation solar radio telescope being planed, which allows to image the Sun and its atmosphere over an ultra-wide frequency range of $50 \mathrm{MHz}$ to more than $20 \mathrm{GHz}$ along with simultaneously high spatial, time, and spectral resolutions. 
On the other end of the electromagnetic spectrum, studies at X-ray wavelengths naturally complement those in radio wavelengths, since they originate from the same population of radio-emitting energetic electrons that are accelerated from a common energy release site. For example, the type-III-burst-emitting electron beams can also produce X-ray emission through their interactions with the ambient plasma. X-ray emissions are the most profound at the footpoints of flaring loops when the downwardgoing beams reach the dense chromosphere. These emissions usually dominate the observed X-ray spectra, and make the much weaker immediate X-ray emission from the propagating electron beams very difficult to detect. The ongoing Focusing Optics X-ray Solar Imager (FOXSI) experiments (Krucker et al., 2011) and the recently launched Nuclear Spectroscopic Telescope Array (NuSTAR; Harrison et al., 2013) provide improved sensitivity and/or dynamic range over the current instruments, making it possible to observe X-ray emission from propagating electron beams directly. Future projects may involve coordinated observations of the $V L A$ and FOXSI/NuSTAR to trace the propagating electron beams from the flare energy release site in both radio and X-ray wavelengths. Another potential project is to obtain energetic electron energy spectra from spatially resolved radio and X-ray spectra (from, e.g., EOVSA and RHESSI), based on a detailed understanding of the relevant radio and X-ray emission mechanism(s). The resulting spatially resolved electron energy spectra are critical in understanding the energy release, particle acceleration and transport processes in solar flares. 


\section{References}

Ackermann, M., Ajello, M., Allafort, A., et al. 2012, ApJ, 745, 144

Acton, L. W., Feldman, U., Bruner, M. E., et al. 1992, PASJ, 44, L71

Aharonian, F. A., \& Atoyan, A. M. 1981, Ap\&SS, 79, 321

Altyntsev, A. T., Kuznetsov, A. A., Meshalkina, N. S., Rudenko, G. V., \& Yan, Y. 2005, A\&A, 431, 1037

Antolin, P., \& Rouppe van der Voort, L. 2012, ApJ, 745, 152

Aschwanden, M. J. 2001, ApJ, 560, 1035

Aschwanden, M. J., Bastian, T. S., Benz, A. O., \& Brosius, J. W. 1992, ApJ, 391, 380

Aschwanden, M. J., \& Benz, A. O. 1997, ApJ, 480, 825

Aschwanden, M. J., Benz, A. O., Dennis, B. R., \& Schwartz, R. A. 1995a, ApJ, 455, 347

Aschwanden, M. J., Boerner, P., Schrijver, C. J., \& Malanushenko, A. 2013, Sol. Phys., 283, 5

Aschwanden, M. J., Schwartz, R. A., \& Alt, D. M. 1995b, ApJ, 447, 923 
Aschwanden, M. J., Wiehl, H. J., Benz, A. O., \& Kane, S. R. 1985, Sol. Phys., 97, 159

Aulanier, G., DeLuca, E. E., Antiochos, S. K., McMullen, R. A., \& Golub, L. 2000, ApJ, 540, 1126

Aurass, H., \& Klein, K.-L. 1997, A\&AS, 123, 279

Aurass, H., Klein, K.-L., Zlotnik, E. Y., \& Zaitsev, V. V. 2003, A\&A, 410, 1001

Bain, H. M., Krucker, S., Glesener, L., \& Lin, R. P. 2012, ApJ, 750, 44

Bale, S. D., Reiner, M. J., Bougeret, J.-L., et al. 1999, Geophys. Res. Lett., 26, 1573

Bárta, M., \& Karlický, M. 2006, A\&A, 450, 359

Bastian, T. S. 1994a, ApJ, 426, 774

-. 1994b, Space Sci. Rev., 68, 261

Bastian, T. S. 2003, in Society of Photo-Optical Instrumentation Engineers (SPIE) Conference Series, Vol. 4853, Society of Photo-Optical Instrumentation Engineers (SPIE) Conference Series, ed. S. L. Keil \& S. V. Avakyan, 98-110

Bastian, T. S. 2004, in Solar and Space Weather Radiophysics: Current Status and Future Developments, ed. D. E. Gary \& C. U. Keller, Vol. 314 (Springer), 47-69

Bastian, T. S. 2006, in Astronomical Society of the Pacific Conference Series, Vol. 358, Astronomical Society of the Pacific Conference Series, ed. R. Casini \& B. W. Lites, 173

Bastian, T. S., Benz, A. O., \& Gary, D. E. 1998, ARA\&A, 36, 131 
Bastian, T. S., \& Bookbinder, J. A. 1987, Nature, 326, 678

Battaglia, M., \& Benz, A. O. 2006, A\&A, 456, 751

-. 2008, A\&A, 487, 337

-. 2009, A\&A, 499, L33

Benz, A., ed. 2002, Astrophysics and Space Science Library, Vol. 279, Plasma Astrophysics, second edition

Benz, A. O. 1985, Sol. Phys., 96, 357

-. 1986, Sol. Phys., 104, 99

—. 2008, Living Reviews in Solar Physics, 5, 1

Benz, A. O., Csillaghy, A., \& Aschwanden, M. J. 1996, A\&A, 309, 291

Benz, A. O., Grigis, P. C., Csillaghy, A., \& Saint-Hilaire, P. 2005, Sol. Phys., 226, 121

Benz, A. O., Magun, A., Stehling, W., \& Su, H. 1992, Sol. Phys., 141, 335

Benz, A. O., Saint-Hilaire, P., \& Vilmer, N. 2002, A\&A, 383, 678

Blumenthal, G. R., \& Gould, R. J. 1970, Reviews of Modern Physics, 42, 237

Bougeret, J. L., Goetz, K., Kaiser, M. L., et al. 2008, Space Sci. Rev., 136, 487

Brown, D. S., Nightingale, R. W., Alexander, D., et al. 2003, Sol. Phys., 216, 79

Brown, J. C. 1971, Sol. Phys., 18, 489

Brown, J. C., Kašparová, J., Massone, A. M., \& Piana, M. 2008, A\&A, 486, 1023 
Brunetti, G. 2000, Astroparticle Physics, 13, 107

Carmichael, H. 1964, NASA Special Publication, 50, 451

Carrington, R. C. 1859, MNRAS, 20, 13

Charbonneau, P., Christensen-Dalsgaard, J., Henning, R., et al. 1999, ApJ, 527, 445

Chen, B., \& Bastian, T. S. 2012, ApJ, 750, 35

Chen, B., Bastian, T. S., Gary, D. E., \& Jing, J. 2011, ApJ, 736, 64

Chen, B., Bastian, T. S., White, S. M., et al. 2013, ApJ, 763, L21

Chen, B., \& Yan, Y. 2007, Sol. Phys., 246, 431

-. 2008, ApJ, 689, 1412

Chernov, G. P. 1976, Soviet Ast., 20, 582

—. 1990, Sol. Phys., 130, 75

—. 2005, Plasma Physics Reports, 31, 314

—. 2006, Space Sci. Rev., 127, 195

Chernov, G. P., Klein, K.-L., Zlobec, P., \& Aurass, H. 1994, Sol. Phys., 155, 373

Chernov, G. P., Markeev, A. K., Poquerusse, M., et al. 1998, A\&A, 334, 314

Chernov, G. P., Poquerusse, M., Bougeret, J.-L., \& Zlobec, P. 2001, Radio Science, 36,1745

Chernov, G. P., Sych, R. A., Yan, Y., et al. 2006, Sol. Phys., 237, 397

Chernov, G. P., Yan, Y. H., \& Fu, Q. J. 2003, A\&A, 406, 1071 
Chernov, G. P., Yan, Y. H., Fu, Q. J., \& Tan, C. M. 2005, A\&A, 437, 1047

Cirtain, J., Golub, L., Winebarger, A., et al. 2013, Nature, 493, 501

Cliver, E. W., Dennis, B. R., Kiplinger, A. L., et al. 1986, ApJ, 305, 920

Cliver, E. W., White, S. M., \& Balasubramaniam, K. S. 2011, ApJ, 743, 145

Cranmer, S. R. 2002, Space Sci. Rev., 101, 229

DeForest, C. E. 2007, ApJ, 661, 532

Deng, X. H., \& Matsumoto, H. 2001, Nature, 410, 557

Dennis, B. R. 1988, Sol. Phys., 118, 49

Dennis, B. R., Benz, A. O., Ranieri, M., \& Simnett, G. M. 1984, Sol. Phys., 90, 383

Dennis, B. R., \& Zarro, D. M. 1993, Sol. Phys., 146, 177

Dere, K. P., Landi, E., Mason, H. E., Monsignori Fossi, B. C., \& Young, P. R. 1997, A\&AS, 125, 149

Dere, K. P., Landi, E., Young, P. R., et al. 2009, A\&A, 498, 915

Dermer, C. D., \& Ramaty, R. 1986, ApJ, 301, 962

Drake, J. F., Swisdak, M., Che, H., \& Shay, M. A. 2006, Nature, 443, 553

Dulk, G. A. 1985, ARA\&A, 23, 169

Dulk, G. A., Kiplinger, A. L., \& Winglee, R. M. 1992, ApJ, 389, 756

Dulk, G. A., \& Marsh, K. A. 1982, ApJ, 259, 350

Dulk, G. A., \& Suzuki, S. 1980, A\&A, 88, 203 
Elgaröy, O. 1959, Nature, 184, 887

Elgarøy, Ø. 1982, Institute of Theoretical Astrophysics, Blindern-Oslo, 53

Fan, Y. 2009, Living Reviews in Solar Physics, 6, 4

Fleishman, G. D., \& Kuznetsov, A. A. 2010, ApJ, 721, 1127

Fleishman, G. D., \& Melnikov, V. F. 2003, ApJ, 587, 823

Fleishman, G. D., Nita, G. M., \& Gary, D. E. 2009, ApJ, 698, L183

Fleishman, G. D., Stepanov, A. V., \& Yurovsky, Y. F. 1994, Sol. Phys., 153, 403

Fletcher, L., Hannah, I. G., Hudson, H. S., \& Metcalf, T. R. 2007, ApJ, 656, 1187

Fletcher, L., \& Hudson, H. S. 2008, ApJ, 675, 1645

Forbes, T. G., \& Acton, L. W. 1996, ApJ, 459, 330

Frost, K. J., \& Dennis, B. R. 1971, ApJ, 165, 655

Gary, D. E., Fleishman, G. D., \& Nita, G. M. 2013, Sol. Phys., arXiv:1303.1573

Gary, D. E., \& Hurford, G. J. 1994, ApJ, 420, 903

Gary, D. E., \& Hurford, G. J. 2004, in Solar and Space Weather Radiophysics: Current Status and Future Developments, ed. D. E. Gary \& C. U. Keller, Vol. 314 (Springer), 71-87

Gary, D. E., Hurford, G. J., Nita, G. M., et al. 2011, in AAS/Solar Physics Division Abstracts \#42, 102

Georgoulis, M. K., \& LaBonte, B. J. 2006, ApJ, 636, 475 
Ginzburg, V. L., \& Zheleznyakov, V. V. 1958, Soviet Ast., 2, 653

Golub, L., Deluca, E., Austin, G., et al. 2007, Sol. Phys., 243, 63

Grebinskij, A., Bogod, V., Gelfreikh, G., et al. 2000, A\&AS, 144, 169

Hagyard, M. J., Venkatakrishnan, P., \& Smith, Jr., J. B. 1990, ApJS, 73, 159

Hankins, T. H., \& Eilek, J. A. 2007, ApJ, 670, 693

Hara, H., Tsuneta, S., Lemen, J. R., Acton, L. W., \& McTiernan, J. M. 1992, PASJ, 44, L135

Harrison, F. A., Craig, W. W., Christensen, F. E., et al. 2013, ApJ, 770, 103

Haug, E. 1975a, Zeitschrift Naturforschung Teil A, 30, 1099

-. 1975b, Sol. Phys., 45, 453

Heyvaerts, J., Priest, E. R., \& Rust, D. M. 1977, ApJ, 216, 123

Hirayama, T. 1974, Sol. Phys., 34, 323

Holman, G. D. 2003, ApJ, 586, 606

Holman, G. D., Eichler, D., \& Kundu, M. R. 1980, in IAU Symposium, Vol. 86, Radio Physics of the Sun, ed. M. R. Kundu \& T. E. Gergely, 457-459

Holman, G. D., Sui, L., Schwartz, R. A., \& Emslie, A. G. 2003, ApJ, 595, L97

Hoyng, P., Duijveman, A., Machado, M. E., et al. 1981, ApJ, 246, L155

Hurford, G. J., Schmahl, E. J., Schwartz, R. A., et al. 2002, Sol. Phys., 210, 61

Hurley, K., Boggs, S. E., Smith, D. M., et al. 2005, Nature, 434, 1098 
Ji, H., Cao, W., \& Goode, P. R. 2012, ApJ, 750, L25

Jones, F. C. 1968, Physical Review, 167, 1159

Kaiser, M. L., Kucera, T. A., Davila, J. M., et al. 2008, Space Sci. Rev., 136, 5

Kane, S. R. 1974, in IAU Symposium, Vol. 57, Coronal Disturbances, ed. G. A. Newkirk, 105-141

Kane, S. R. 1981, ApJ, 247, 1113

Karlický, M., \& Bárta, M. 2007, A\&A, 464, 735

Kiplinger, A. L. 1995, ApJ, 453, 973

Klein, K.-L. 1987, A\&A, 183, 341

Kliem, B., Karlický, M., \& Benz, A. O. 2000, A\&A, 360, 715

Koch, H. W., \& Motz, J. W. 1959, Reviews of Modern Physics, 31, 920

Kontar, E. P., Emslie, A. G., Massone, A. M., et al. 2007, ApJ, 670, 857

Kontar, E. P., MacKinnon, A. L., Schwartz, R. A., \& Brown, J. C. 2006, A\&A, 446, 1157

Kopp, R. A., \& Pneuman, G. W. 1976, Sol. Phys., 50, 85

Korchak, A. A. 1967, Soviet Ast., 11, 258

—. 1971, Sol. Phys., 18, 284

Kosugi, T., Sakao, T., Masuda, S., et al. 1992, PASJ, 44, L45

Kosugi, T., Matsuzaki, K., Sakao, T., et al. 2007, Sol. Phys., 243, 3 
Krall, K. R., Smith, Jr., J. B., Hagyard, M. J., West, E. A., \& Cummings, N. P. 1982, Sol. Phys., 79, 59

Krucker, S., Hudson, H. S., Glesener, L., et al. 2010, ApJ, 714, 1108

Krucker, S., Hurford, G. J., MacKinnon, A. L., Shih, A. Y., \& Lin, R. P. 2008a, ApJ, 678, L63

Krucker, S., Kontar, E. P., Christe, S., \& Lin, R. P. 2007a, ApJ, 663, L109

Krucker, S., White, S. M., \& Lin, R. P. 2007b, ApJ, 669, L49

Krucker, S., Battaglia, M., Cargill, P. J., et al. 2008b, A\&A Rev., 16, 155

Krucker, S., Christe, S., Glesener, L., et al. 2011, in Society of Photo-Optical Instrumentation Engineers (SPIE) Conference Series, Vol. 8147, Society of Photo-Optical Instrumentation Engineers (SPIE) Conference Series

Kuijpers, J. M. E. 1975, PhD thesis, Utrecht, Rijksuniversiteit, Doctor in de Wiskunde en Natuurwetenschappen Dissertation, 1975. 72 p. Research supported by the Nederlandse Organisatie voor Zuiver-Wetenschappelijk Onderzoek.

Kuznetsov, A. A. 2008, Sol. Phys., 253, 103

Kuznetsov, A. A., \& Tsap, Y. T. 2007, Sol. Phys., 241, 127

LaBelle, J., Treumann, R. A., Yoon, P. H., \& Karlicky, M. 2003, ApJ, 593, 1195

Laptukhov, A. I., \& Chernov, G. P. 2006, Plasma Physics Reports, 32, 866

Leblanc, Y., Dulk, G. A., \& Bougeret, J.-L. 1998, Sol. Phys., 183, 165

Ledenev, V. G., Yan, Y., \& Fu, Q. 2006, Sol. Phys., 233, 129 
Lee, J. 2007, Space Sci. Rev., 133, 73

Lemen, J. R., Title, A. M., Akin, D. J., et al. 2012, Sol. Phys., 275, 17

Lin, R. P., \& Hudson, H. S. 1976, Sol. Phys., 50, 153

Lin, R. P., \& Schwartz, R. A. 1987, ApJ, 312, 462

Lin, R. P., Schwartz, R. A., Pelling, R. M., \& Hurley, K. C. 1981, ApJ, 251, L109

Lin, R. P., Dennis, B. R., Hurford, G. J., et al. 2002, Sol. Phys., 210, 3

Lin, R. P., Krucker, S., Hurford, G. J., et al. 2003, ApJ, 595, L69

Liu, C., Lee, J., Gary, D. E., \& Wang, H. 2007a, ApJ, 658, L127

Liu, Z. 2007, PhD thesis, New Jersey Institute of Technology and Rutgers, the State University of New Jersey

Liu, Z., Gary, D. E., Nita, G. M., White, S. M., \& Hurford, G. J. 2007b, PASP, 119, 303

MacKinnon, A. L., \& Mallik, P. C. V. 2010, A\&A, 510, A29

Maehara, H., Shibayama, T., Notsu, S., et al. 2012, Nature, 485, 478

Magara, T., Mineshige, S., Yokoyama, T., \& Shibata, K. 1996, ApJ, 466, 1054

Mann, G., Jansen, F., MacDowall, R. J., Kaiser, M. L., \& Stone, R. G. 1999, A\&A, 348,614

Massone, A. M., Emslie, A. G., Kontar, E. P., et al. 2004, ApJ, 613, 1233

Masuda, S., Kosugi, T., Hara, H., Tsuneta, S., \& Ogawara, Y. 1994, Nature, 371, 495 
McKinnon, M., \& Perley, R. A. 2001, The VLA Expansion Project: Construction Project Book, Tech. rep., National Radio Astronomy Observatory

McTiernan, J. M., Fisher, G. H., \& Li, P. 1999, ApJ, 514, 472

Melrose, D. B., \& Dulk, G. A. 1982, ApJ, 259, 844

Metcalf, T. R., Leka, K. D., Barnes, G., et al. 2006, Sol. Phys., 237, 267

Miller, J. A., Cargill, P. J., Emslie, A. G., et al. 1997, J. Geophys. Res., 102, 14631

Moore, R. L., Cirtain, J. W., Sterling, A. C., \& Falconer, D. A. 2010, ApJ, 720, 757

Moser, A. L., \& Bellan, P. M. 2012, Nature, 482, 379

Moskalenko, I. V., \& Strong, A. W. 2000, ApJ, 528, 357

Neupert, W. M. 1968, ApJ, 153, L59

Øieroset, M., Phan, T. D., Fujimoto, M., Lin, R. P., \& Lepping, R. P. 2001, Nature, 412,414

Ott, J., \& Kern, J. 2012, CASA Reference Manual \& Cookbook, National Radio Astronomy Observatory

Paesold, G., Benz, A. O., Klein, K.-L., \& Vilmer, N. 2001, A\&A, 371, 333

Palmer, D. M., Barthelmy, S., Gehrels, N., et al. 2005, Nature, 434, 1107

Payne-Scott, R. 1949, Australian Journal of Scientific Research A Physical Sciences, 2,214

Payne-Scott, R., Yabsley, D. E., \& Bolton, J. G. 1947, Nature, 160, 256

Pe'er, A., \& Waxman, E. 2005, ApJ, 628, 857 
Perley, R. A., \& Bastian, T. S. 2004, EVLA MEMO 70: Desiderata for Solar Observing with the EVLA, Tech. rep., National Radio Astronomy Observatory

Perley, R. A., Chandler, C. J., Butler, B. J., \& Wrobel, J. M. 2011, ApJ, 739, L1

Pesnell, W. D., Thompson, B. J., \& Chamberlin, P. C. 2012, Sol. Phys., 275, 3

Petrosian, V. 1981, ApJ, 251, 727

Phan, T. D., Gosling, J. T., Davis, M. S., et al. 2006, Nature, 439, 175

Ramaty, R. 1969, ApJ, 158, 753

Raulin, J. P., Kundu, M. R., Hudson, H. S., Nitta, N., \& Raoult, A. 1996, A\&A, 306, 299

Reale, F. 2010, Living Reviews in Solar Physics, 7, 5

Rieger, E., \& Marschhäuser, H. 1991, in Max '91/SMM Solar Flares: Observations and Theory, ed. R. M. Winglee \& A. L. Kiplinger, 68

Rosenberg, H. 1972, Sol. Phys., 25, 188

Sakao, T. 1994, PhD thesis, (University of Tokyo), (1994)

Scherrer, P. H., Schou, J., Bush, R. I., et al. 2012, Sol. Phys., 275, 207

Schmidt, J. M., \& Cairns, I. H. 2012, Journal of Geophysical Research (Space Physics), 117, 4106

Schwartz, R. A., Csillaghy, A., Tolbert, A. K., et al. 2002, Sol. Phys., 210, 165

Slottje, C. 1972, Sol. Phys., 25, 210

Spicer, D. S., \& Sudan, R. N. 1984, ApJ, 280, 448 
Spiegel, E. A., \& Zahn, J.-P. 1992, A\&A, 265, 106

Sturrock, P. A. 1966, Nature, 211, 695

Su, Y., Golub, L., van Ballegooijen, A., et al. 2007, PASJ, 59, 785

Sui, L., \& Holman, G. D. 2003, ApJ, 596, L251

Tan, B. 2010, Ap\&SS, 325, 251

Tandberg-Hanssen, E., \& Emslie, A. G. 1988, The physics of solar flares (Cambridge University Press)

Tavani, M., Bulgarelli, A., Piano, G., et al. 2009, Nature, 462, 620

Taylor, G. B., Carilli, C. L., \& Perley, R. A., eds. 1999, Astronomical Society of the Pacific Conference Series, Vol. 180, Synthesis Imaging in Radio Astronomy II

Temmer, M., Veronig, A. M., Vršnak, B., \& Miklenic, C. 2007, ApJ, 654, 665

Terasawa, T., Tanaka, Y. T., Takei, Y., et al. 2005, Nature, 434, 1110

Treumann, R. A. 2006, A\&A Rev., 13, 229

Trottet, G., Vilmer, N., Barat, C., et al. 1998, A\&A, 334, 1099

Tsuneta, S., Hara, H., Shimizu, T., et al. 1992, PASJ, 44, L63

Tsuneta, S., Ichimoto, K., Katsukawa, Y., et al. 2008, Sol. Phys., 249, 167

van den Oord, G. 1994, Fragmented energy release in sun and stars: the interface between MHD and plasma physics (Kluwer Academic Publishers)

van den Oord, G. H. J. 1990, A\&A, 234, 496 
Veronig, A., Vršnak, B., Dennis, B. R., et al. 2002, A\&A, 392, 699

Veronig, A. M., \& Brown, J. C. 2004, ApJ, 603, L117

Vestrand, W. T. 1988, Sol. Phys., 118, 95

Vilmer, N., MacKinnon, A. L., Trottet, G., \& Barat, C. 2003, A\&A, 412, 865

Wheatland, M. S., Sturrock, P. A., \& Roumeliotis, G. 2000, ApJ, 540, 1150

White, S. M., Benz, A. O., Christe, S., et al. 2011, Space Sci. Rev., 159, 225

Wiegelmann, T. 2004, Sol. Phys., 219, 87

Wiegelmann, T., Inhester, B., \& Sakurai, T. 2006, Sol. Phys., 233, 215

Wild, J. P., \& McCready, L. L. 1950, Australian Journal of Scientific Research A Physical Sciences, 3, 387

Wild, J. P., Murray, J. D., \& Rowe, W. C. 1954, Australian Journal of Physics, 7, 439

Wild, J. P., \& Smerd, S. F. 1972, ARA\&A, 10, 159

Winglee, R. M., \& Dulk, G. A. 1986, ApJ, 307, 808

Woods, T. N., Eparvier, F. G., Hock, R., et al. 2012, Sol. Phys., 275, 115

Wuelser, J.-P., Lemen, J. R., Tarbell, T. D., et al. 2004, in Society of Photo-Optical Instrumentation Engineers (SPIE) Conference Series, Vol. 5171, Society of PhotoOptical Instrumentation Engineers (SPIE) Conference Series, ed. S. Fineschi \& M. A. Gummin, 111-122

Young, C. W., Spencer, C. L., Moreton, G. E., \& Roberts, J. A. 1961, ApJ, 133, 243 
Zaitsev, V. V., \& Stepanov, A. V. 1983, Sol. Phys., 88, 297

Zharkova, V. V., Arzner, K., Benz, A. O., et al. 2011, Space Sci. Rev., 159, 357

Zheleznyakov, V. V., \& Zlotnik, E. I. 1975a, Sol. Phys., 43, 431

Zheleznyakov, V. V., \& Zlotnik, E. Y. 1975b, Sol. Phys., 44, 461

Zlotnik, E. Y. 2009, Central European Astrophysical Bulletin, 33, 281

Zlotnik, E. Y., Zaitsev, V. V., Aurass, H., \& Mann, G. 2009, Sol. Phys., 255, 273

Zlotnik, E. Y., Zaitsev, V. V., Aurass, H., Mann, G., \& Hofmann, A. 2003, A\&A, 410, 1011 